\title{
Dielektrische Wellenleitergitter in Resonanz:
}

\section{Theorie, Charakterisierung und Anwendung}

\author{
Dissertation \\ zur Erlangung des mathematisch-naturwissenschaftlichen Doktorgrades \\ „Doctor rerum naturalium“ \\ der Georg-August-Universität Göttingen
}

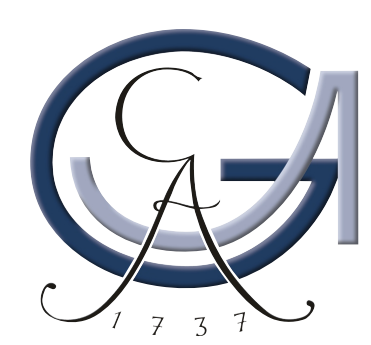

vorgelegt von

\author{
ANDRÉ SELLE \\ (Diplom-Physiker) \\ aus \\ Dessau-Roßlau
}

Göttingen 2009 
D7

Referent: Prof. Dr. Gerd Marowsky

Korreferent: Prof. DR. WERnER LAUTERBORN

Tag der mündlichen Prüfung: 19. November 2008 
»Weisheit ist nicht das Ergebnis der Schulbildung, sondern des lebenslangen Versuchs, sie zu erwerben. «

Albert Einstein 



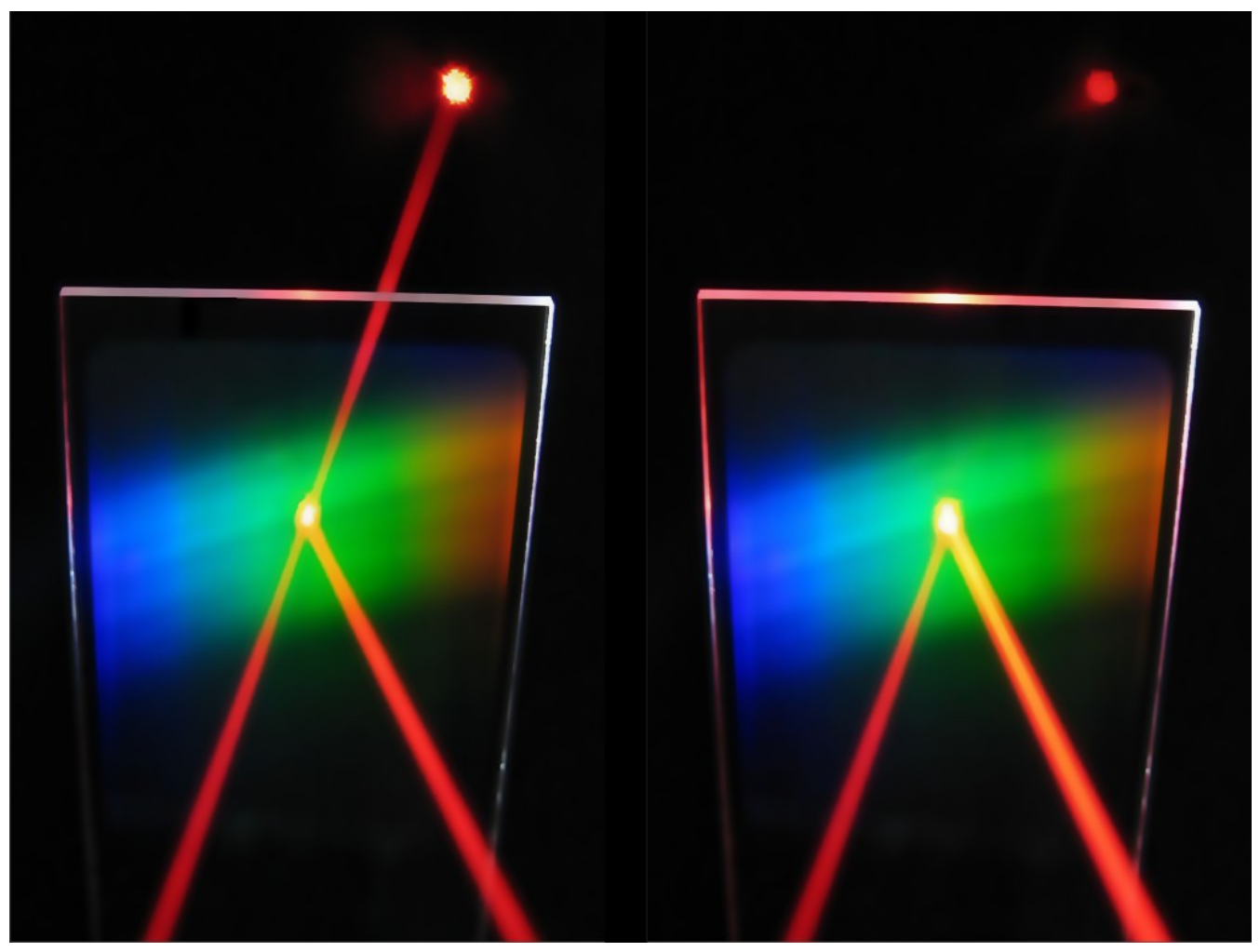





\section{Kurzfassung}

Die strukturelle Verknüpfung eines planaren Wellenleiters mit einem dielektrischen Beugungsgitter ermöglicht es, polarisiertes monochromatisches Licht unter einem bestimmten Winkel in die Verbundstruktur ein- und vollständig wieder auszukoppeln. Diese Eigenschaft sog. dielektrischer Wellenleitergitter wird als elektromagnetische Resonanz verstanden und besitzt drei wesentliche Merkmale: Nahezu vollständige Unterdrückung des Anregungslichts in Transmission; gleichzeitiger Anstieg der Reflektivität auf annähernd 100\%; Auftreten stark erhöhter, aber schnell abklingender (evaneszenter) Felder an der Oberfläche des Wellenleiters.

Um die Resonanz von Wellenleitergittern besser zu verstehen, werden vorstrukturierte Substrate mit optisch hoch-brechenden Materialien beschichtet und anschließend mittels Laserlicht verschiedener Wellenlängen charakterisiert. Das Resonanzverhalten der gefertigten Proben wird dabei in Transmission und Reflexion sowohl spektral als auch winkelabhängig untersucht und mit Berechnungen auf der Grundlage analytischer und numerischer Methoden verglichen. Zusätzlich zu den Proben mit rein passivem Verhalten wird mithilfe eines porösen Wellenleiters ein neuer Freiheitsgrad erzeugt, der es erlaubt, den Brechungsindex der Schicht und damit die Resonanz durch bloße Änderung der Temperatur zu verschieben.

Um die unter Resonanzbedingungen auftretende Felderhöhung zu bestimmen, werden Markerfarbstoffe auf der Oberfläche der Wellenleitergitter aufgebracht und mithilfe eines Ultrakurzpuls-Lasers durch Zwei-Photonen-Absorptionsprozesse zur Emission von Licht (Fluoreszenz) angeregt. Dabei werden Verstärkungen der Fluoreszenz von deutlich über zwei Größenordnungen gegenüber nicht-resonanter Anregung beobachtet; wobei ein Nachweis noch bei zehn Farbstoffmolekülen auf einem Quadratmikrometer möglich ist. Den Abschluss der Untersuchungen stellt die Fluoreszenzlichtdetektion einer körperverwandten Aminosäuresequenz in nativer Umgebung dar. Die daraus resultierenden Ergebnisse unterstreichen das Anwendungspotential der Wellenleitergitter als Sensorplattform für die Biochemie. 



\section{Abstract}

The combination of a planar waveguide and a one-dimensional diffraction grating allows to couple polarized monochromatic light under a given incident angle into and totally out of the all-dielectric compound structure. This optical property of a so-called dielectric waveguide-grating is ascribed to an electromagnetic resonance that exhibits three significant and remarkable features: A nearly complete suppression of the incident light in transmission geometry; a simultaneous increase of the reflectivity to almost $100 \%$; an emergence of strongly enhanced but rapidly decaying (evanescent) waves at the boundary interfaces of the waveguide layer.

To understand the resonance of waveguide-gratings, substrates with a prestructured grating profile are coated with high refractive index waveguide materials. Subsequently, the manufactured waveguide-grating samples are characterized by laser light of different wavelengths. In order to analyze the resonance behavior, the spectrum as well as angular distribution of the transmitted and reflected light is detected and all of the experimental results are compared with numerical calculations. In addition to the samples with a purely passive structure, a specific porous waveguide material with a temperature-dependent refractive index allows a spectral shift of the resonance simply by changing the temperature of the sample.

Furthermore, to determine the field enhancement near the waveguide interfaces, fluorescent dye molecules are applied on the surface of a waveguide-grating and are stimulated with an ultra-short pulse laser. Due to two-photon absorption processes of the dye molecules fluorescent light from the surface is emitted. Under resonance condition, a fluorescence enhancement by over two orders of magnitudes compared to a non-resonant excitation is observed. The resonant fluorescence excitation is still possible with a detection limit of only ten dye molecules on a square micrometer. As a result, one is able to successfully measure the enhanced fluorescence light of a labeled human self-peptide in an aqueous environment. This strongly suggests the waveguide-grating as an enhancement sensor platform in biotechnology. 



\section{Publikationen}

Bereits im Vorfeld dieser Doktorschrift sind wissenschaftliche Beiträge in Form von Originalarbeiten in referierten Fachzeitschriften und Tagungsbänden unter eigener Mitwirkung publiziert worden. Darüber hinaus sind Teile dieser Arbeit in Vorträgen oder auf Postern verwendet worden.

\section{Originalbeiträge in Fachzeitschriften:}

[1] Kappel, C. ; Selle, A. ; Bader, M. A. ; Marowsky, G.: Resonant double-grating waveguide structures as inverted Fabry-Perot interferometers. In: Journal of Optical Society of America B 21 (2004), Nr. 6, S. 1127-1136

[2] Kappel, C. ; Selle, A. ; Fricke-Begemann, T. ; Bader, M. A. ; Marowsky, G.: Giant enhancement of two-photon fluorescence induced by resonant double grating waveguide structures. In: Applied Physics B: Lasers and Optics 79 (2004), Nr. 5, S. 531-534

[3] Soria, S. ; Thayil, A. ; Badenes, G. ; Bader, M. A. ; Selle, A. ; MARowsky, G.: Resonant double grating waveguide structures as enhancement platforms for two-photon fluorescence excitation. In: Applied Physics Letters 87 (2005), Nr. 8. - Art. 081109

[4] Selle, A. ; Kappel, C. ; Bader, M. A. ; Marowsky, G. ; Winkler, K. ; Alexiev, U.: Picosecond-pulse-induced two-photon fluorescence enhancement in biological material by application of grating waveguide structures. In: Optics Letters 30 (2005), Nr. 13, S. $1683-1685$ 
[5] Bader, M. A. ; Kappel, C. ; Selle, A. ; Ihlemann, J. ; NG, M. L. ; Herman, P. R.: F $_{2}$-laser-machined submicrometer gratings in thin dielectric films for resonant grating waveguide applications. In: Applied Optics 45 (2006), Nr. 25, S. 6586-6590

[6] Delmdahl, R. F. ; Spiecker, G. ; Bader, M. A. ; Selle, A.: Spectral resolution analysis of resonant grating waveguides. In: $M a-$ terialwissenschaft und Werkstofftechnik 38 (2007), Nr. 3, S. 218-221

[7] Bader, M. A. ; Selle, A. ; Stenzel, O. ; Delmdahl, R. ; SpieCKER, G. ; Fischer, C.: High spectral resolution analysis of tunable narrowband resonant grating waveguide structures. In: Applied Physics B 89 (2007), Nr. 2-3, S. 151-154

\section{Originalbeiträge in Tagungsbänden:}

[1] Kappel, C. ; Selle, A. ; Fricke-Begemann, T. ; Bader, M. A. ; Marowsky, G.: Double grating waveguide structures : 350-fold enhancement of two-photon fluorescence applying ultrashort pulses. In: Moreno-Bondi, M. C. (Hrsg.) ; Orellana, G. (Hrsg.): Sensors and Actuators B: Chemical (Proceedings of the 7th European Conference on Optical Chemical Sensors and Biosensors - EUROPT(R)ODE VII, Madrid, Spanien, 4.-7. April 2004). Bd. 107, Nr. 1. Amsterdam : North-Holland/Elsevier Press, Mai 2005. - ISSN 0925-4005, S. 135-139

[2] Kappel, C. ; Selle, A. ; Bader, M. A. ; Marowsky, G.: Ultrashort-pulse behavior in resonant reflection filters. In: ARMITAGE, J. C. (Hrsg.) ; FAfArd, S. (Hrsg.) ; Lessard, R. A. (Hrsg.) ; Lampropoulos, G. A. (Hrsg.): Proceedings of the SPIE (Photonics North 2004, Ottawa, Ontario, Kanada, 27.-29. September 2004). Bd. 5577 : Optical Components and Devices. Bellingham (Washington) : SPIE Press, Dezember 2004. - ISBN 0-8194-5533-4, S. 716-723 
[3] Bader, M. A. ; Kappel, C. ; Selle, A. ; Ihlemann, J. ; NG, M. L. ; Herman, P. R.: Fabrication of sub-micron gratings in ultrathin films by $157-\mathrm{nm}$ laser ablation and their application as grating waveguide structures. In: Armitage, J. C. (Hrsg.) ; Lessard, R. A. (Hrsg.) ; Lampropoulos, G. A. (Hrsg.): Proceedings of SPIE (Photonics North 2004, Ottawa, Ontario, Kanada, 27.-29. September 2004). Bd. 5578 : Photonic Applications in Astronomy, Biomedicine, Imaging, Materials Processing, and Education. Bellingham (Washington) : SPIE Press, 2004. - ISBN 0-8194-5533-4, S. 559-567

[4] Marowsky, G. ; Bader, M. A. ; Selle, A. ; Kappel, C. ; Fricke-Begemann, T. ; Meinertz, J. ; Ihlemann, J.: Neue Perspektiven der Zwei-Photonen-Technologie. In: Beckmann, D. (Hrsg.) ; MeIster, M. (Hrsg.) ; Institut für Bioprozess- und Analysenmesstechnik (Veranst.): Technische Systeme für Biotechnologie und Umwelt (Tagungsband des 12. Heiligenstädter Kolloquium, Heiligenstadt, Deutschland, 2\%.-29. September 2004). Heiligenstadt, 2004. - ISBN 3-00-015042-0, S. 119-124

[5] Fricke-Begemann, T. ; Bader, M. A. ; Ihlemann, J. ; KapPel, C. ; Meinertz, J. ; Selle, A.: Two-photon fluorescence: large area excitation and enhanced sensitivity using waveguide structures. In: DGaO-Proceedings (106. Joint Conference of German Society of Applied Optics (DGaO) and Section of Optics of the Polish Physical Society, Wroclaw, Polen, 17.-21. Mai 2005). 2005. - Art. B24.- URL http://www.dgao-proceedings.de/download/106/106_b24.pdf

[6] Thayil, A. ; Soria, S. ; Badenes, G. ; Katchalski, T. ; FrieSem, A. A. ; Selle, A. ; Bader, M. A. ; Marowsky, G.: Twophoton fluorescence spectroscopy by resonant single and double grating waveguide structures. In: Proceedings of OSA (Conference on Lasers and Electro-Optics - CLEO 2005, Baltimore, Maryland, USA, 22.-27. Mai 2005). OSA Press, 2005. - Technical Digest CD-Rom. ISBN 1-55752-770-9, Art. CTuH2 


\section{Poster und Vorträge:}

[1] Kappel, C. ; Selle, A. ; Bader, M. A. ; Marowsky, G.: Resonant single and double-grating waveguide structures as inverted Fabry-Perot interferometers. In: Physikalisch-Technische Bundesanstalt (Veranst.): 14. Norddeutscher Lasertag, Braunschweig, Deutschland, 3. Dezember 2003. - Poster

[2] Winkler, K. ; Selle, A. ; Kappel, C. ; Bader, M. A. ; MaRowsky, G.; Alexiev, U.: Picosecond pulse induced two-photon fluorescence enhancement in biological material applying grating waveguide structures. In: Biophysical Society (Veranst.): 49th Annual Meeting of the Biophysical Society, Long Beach, Kalifornien, USA, 12.-16. Februar 2005. - Vortrag

[3] Winkler, K. ; Selle, A. ; Bader, M. A. ; Marowsky, G. ; Alexiev, U.: Picosecond pulse induced two-photon fluorescence enhancement in biological material applying grating waveguide structures: Towards the elucidation of MHC-Antigen recognition in living cells. In: Molecular basis of signal information and energy transduction in biomolecules (Joint meeting of Swiss and German Biophysicists, Hünfeld, Deutschland, 5.-7. Mai 2005). - Vortrag

\section{Kurzbeitrag:}

[1] Selle, A. ; Bader, M. A. ; Marowsky, G.: Enhancement of two-photon excited fluorescence induced by double grating waveguide structures. In: Molecular basis of signal information and energy transduction in biomolecules (Joint meeting of Swiss and German Biophysicists, Hünfeld, Deutschland, 5.-7. Mai 2005). - Kurzbeitrag 


\section{Inhaltsverzeichnis}

Kurzfassung vii vii v

Abstract ix - ix

Publikationen xi

Inhaltsverzeichnis $\quad x v$

\begin{tabular}{llr}
\hline 1 & Einleitung & 1
\end{tabular}

$1.1 \quad$ Phänomenologische Beschreibung, Namensgebung und Begriffe . . . 1

1.2 Resonante Wellenleitergitter in der Wissenschaft . . . . . . . . . . . . 4

1.3 Motivation, Aufgabenstellung und Aufbau der Arbeit . . . . . . . . 7

2 Theorie zu Wellenleitergittern 9

$2.1 \quad$ Polarisierbarkeit und Totalreflexion elektromagnetischer Wellen . . 9

2.2 Schwingungsmoden in planaren Wellenleitern . . . . . . . . . . . . . 17

2.3 Dielektrisches Beugungsgitter . . . . . . . . . . . . . . . . 20

2.4 Analytische Beschreibung resonanter Wellenleitergitter . . . . . . . 22

$2.4 .1 \quad$ Phasenanpassung resonanter Wellenleitergitter . . . . . . . . 25

2.4 .2 Infinitesimale Betrachtung der Phasenverschiebung . . . . . 31

2.4 .3 Intensitätsverhalten in Transmission und Reflexion . . . . . 35

2.5 Numerische Berechnungen sowie ergänzende Beispielsimulationen mithilfe rigoroser Methoden . . . . . . . . . . . . . . . . . 37

3 Herstellung dielektrischer Wellenleitergitter 43

3.1 Strukturunterschiede dielektrischer Wellenleitergitter . . . . . . . . 43

3.2 Erzeugung der Gitterstrukturen . . . . . . . . . . . . . . . . . . 46

$3.2 .1 \quad$ Lithographische Verfahren . . . . . . . . . . . . . . . . . 47

$3.2 .2 \quad \mathrm{~F}_{2}$-Laserablation $\ldots \ldots \ldots \ldots \ldots$. . . . . . . . . . . 48 
$3.3 \quad$ Fertigung wellenleitender Schichten . . . . . . . . . . . . . . . 52

3.4 Oberflächencharakterisierung gefertigter Wellenleitergitter . . . . . 54

3.5 Zusammenfassung der Strukturdaten gefertigter Wellenleitergitter . 58

4 Optische Charakterisierung resonanter Wellenleitergitter $\quad 61$

4.1 Messung der Resonanzkennlinie . . . . . . . . . . . . . . . . . . 62

4.2 Transmissions- und Reflexionsmessungen . . . . . . . . . . . . . . . 65

4.2.1 Goniometrische Bestimmung der Resonanz bei Anregung mit ultraviolettem Laserlicht . . . . . . . . . . . . . . . . . . 66

4.2.2 Spektrale Bestimmung der Resonanz mit breitbandigem Laserlicht . . . . . . . . . . . . . . . . . 73

$4.2 .3 \quad$ Spektrale Messungen mit monochromatischem Licht . . . . . 80

$4.2 .3 .1 \quad$ Messungen nahe der Rubinlaser-Wellenlänge . . . . 82

4.2.3.2 Durchstimmbares Resonanzverhalten bei Temperaturerhöhung . . . . . . . . . . . . . 87

4.3 Ultrakurzpuls-Analyse der Leckwelle mittels Autokorrelator. . . . . 91

4.4 Diskussion und Ergebnisse . . . . . . . . . . . . . . . . . . . . . . . . 98

5 Wellenleitergitter als Sensorplattform 109

5.1 Zwei-Photonen-Anregung von Fluoreszenzfarbstoffen . . . . . . . . 111

5.2 Zwei-Photonen-Fluoreszenzverstärkung . . . . . . . . . . . . . . . . 114

5.3 Nachweis eines Nonapeptids in wässriger Umgebung . . . . . . . . . 123

$\begin{array}{lll}6 & \text { Zusammenfassung und Ausblick } & 129\end{array}$

\begin{tabular}{ll}
\hline A Anhang & 133
\end{tabular}

\begin{tabular}{lr}
\hline Symbolverzeichnis & 137
\end{tabular}

\begin{tabular}{ll}
\hline Abkürzungsverzeichnis & 141
\end{tabular}

\begin{tabular}{ll}
\hline Abbildungsverzeichnis & 145
\end{tabular}

\begin{tabular}{lr}
\hline Tabellenverzeichnis & 151
\end{tabular}

\begin{tabular}{ll}
\hline Literaturverzeichnis & 153
\end{tabular} 


\section{Einleitung}

Dieses Kapitel leitet in das Thema dieser Arbeit ein und gliedert sich in drei Unterkapitel: Der Abschnitt 1.1 behandelt die phänomenologische Beschreibung des Resonanzeffektes bei dielektrischen Wellenleitergittern und führt darüber hinaus wichtige Namensgebungen und Begriffe ein. Der darauf folgende Abschnitt 1.2 nennt in einem historischen Rückblick die wichtigsten wissenschaftlichen Meilensteine sowie den aktuellen Forschungsstand rund um die Thematik der resonanten Wellenleitergitter. Abschließend widmet sich Abschnitt 1.3 der Zielsetzung sowie dem Aufbau dieser Arbeit.

\subsection{Phänomenologische Beschreibung, Namensgebung und Begriffe}

Die strukturelle Verknüpfung eines planaren Wellenleiters mit einem dielektrischen Beugungsgitter ermöglicht es, polarisiertes, monochromatisches Licht unter einem bestimmten Einfallswinkel in die Verbundstruktur ein- und vollständig wieder auszukoppeln. Diese Eigenschaft sog. dielektrischer Wellenleitergitter (DWG) wird als elektromagnetische Resonanz verstanden, dessen spezifisches Merkmal es ist, das transmittierte Anregungslicht einer bestimmten Wellenlänge vollständig zu unterdrücken - während die Reflektivität dieser Struktur theoretisch auf $100 \%$ ansteigt. Dieses Phänomen im Sinne einer Totalreflexion der 0. Beugungsordnung wird erstmalig von Golubenko u.a. (1985) bei einer sog. Doppel-Gitter-WellenleiterStruktur (DGWS experimentell beobachtet (s.u.).

Diese Totalreflexion kann etwas vereinfacht mithilfe der Wellenoptik beschrieben werden: Mit dem Einfall der elektromagnetischen Welle auf die dielektrischen Gitterstrukturen der DGWS und der dabei auftretenden Gitterbeugung kommt es für genau einen der gebeugten Lichtstrahlen innerhalb der hochbrechenden Schicht 
zur Anregung einer Schwingungsmode, die einer Phasenanpassung im Sinne der Wellenleitung unterliegt und zur einfallenden Welle eine effektive Phasendifferenz von $-\pi / 2$ besitzt. Unter Berücksichtigung der Struktursymmetrie koppelt das Licht jedoch über die ausgedehnten Gitterstrukturen vollständig aus der Struktur wieder aus und erfährt dabei eine weitere Phasenverschiebung um $-\pi / 2$. Im Vergleich zur einfallenden bzw. ungebeugten transmittierten Welle ist die Phase dieser sog. Leckwelle (engl. leaky mode) nun um insgesamt $-\pi$ verschoben, was in Transmission zu einer destruktiven Interferenz und damit zur vollständigen Unterdrückung einer Wellenausbreitung führt (SHARON u. a. 1995; ROSENBLATT u. a. 1997). Anders in Reflexionsgeometrie: Der an der Oberfläche der DGWS reflektierte FRESNEL-Anteil der einfallenden Welle erfährt einen Phasensprung von $\pi$ und interferiert konstruktiv mit der wiederausgekoppelten Leckwelle, so dass in Resonanz die gesamte Energie der einfallenden Welle in Richtung der reflektierten Welle abgegeben wird. Dies geschieht natürlich unter der Annahme idealer Randbedingungen, d.h. der Vernachlässigung von Absorptions- und Streuverlusten.

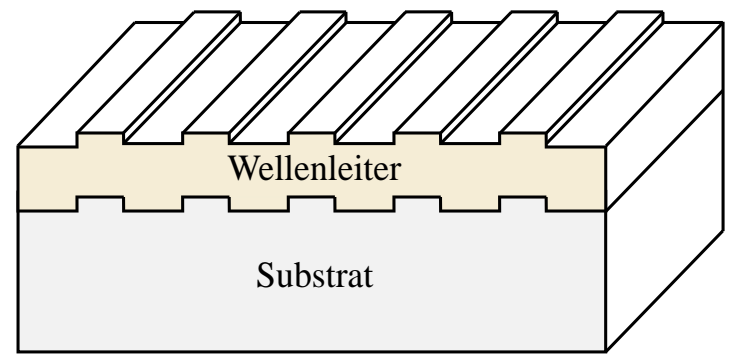

Abb. 1.1: Schematische Darstellung einer Doppel-Gitter-Wellenleiter-Struktur.

Abb. 1.1 zeigt in einer Skizze den strukturellen Aufbau einer DGWS, die aus zwei eindimensionalen Gitterelementen oberhalb und unterhalb der wellenleitenden Schicht sowie einem Substrat besteht. Einzelne Strukturparameter, wie Gitterperiode oder Wellenleiterdicke, stehen dabei im direkten Zusammenhang zum Anregungslicht und sind i. d. R. deutlich kleiner als die Wellenlänge. Für die Anregung von Mono-Moden innerhalb der DGWS mit einer Frequenz aus dem sichtbaren Spektralbereich (VIS) beträgt die Dicke des Wellenleiters typischerweise wenige Hundert Nanometer $\left(10^{-9} \mathrm{~m}\right)$. In einigen Fällen ist der Wellenleiter sogar dünner als die Tiefe der Gitterstege, so dass kein zusammenhängender Bereich im Sinne einer homogenen Schicht mehr besteht. In diesem Fall besteht der wellenleitende Bereich nur aus einer periodischen Brechzahlmodulation und lässt sich deshalb von der Struktur her am ehesten mit einem BRAGGGitter bzw. -Reflektor, welcher die Eigenschaft eines optischen Interferenzfilters 
besitzt, vergleichen. Zur Halterung bzw. Stabilisierung des planaren Wellenleitergitters werden Substratträger mit Dicken bis in den Millimeterbereich benutzt.

Bei dem in dieser Arbeit verwendeten Begriff der DWG handelt es sich um eine allgemeine Bezeichnung der verwendeten Strukturen. Darüber hinaus werden die struktur-bezeichnenden Begriffe DGWS (KAPPEL u. a. 2004b) und GWS (GitterWellenleiter-Struktur) verwendet, wobei letztere im Vergleich zur DGWS nur eine Gitterstruktur enthält (vgl. Abb. 3.1). Die Abkürzung GWS stammt ursprünglich von dem englischen Begriff grating waveguide structure ab, welcher von der Forschergruppe um Asher A. FrIESEm Mitte der 90er Jahre des 20. Jahrhunderts geprägt wird (SHARON u.a. 1995). Durch die Zusammenarbeit mit dem Wissenschaftler HAns-GeORG WEBER etabliert sich der Begriff als Direktübersetzung auch im deutschen Sprachgebrauch (WEBER und PAWLOWSKI 2000). Spätestens mit der Patentanmeldung von DuvENECK u.a. (2001a) wird jedoch die unzureichende Verknüpfung des Begriffs GWS mit dem eigentlichen Resonanzphänomen deutlich: So versteht das Patent die GWS auch als Struktur, die der Lichteinkopplung, der Lichtführung (über getrennte wellenleitende Bereiche) sowie der Wiederauskopplung des Lichts an einem zweiten Gitter dient. Diese Darstellung entspricht jedoch einer Modenanregung planarer Wellenleiter durch ein Koppelgitter, wie sie von DAKSS u. a. 1970 vorgeschlagen wird. Es erscheint daher ungeeignet, die GWS als übergeordneten Begriff in dieser Arbeit zu verwenden.

Aufgrund der Vielzahl von Wissenschaftlern, die sich nach 1985 mit dem Thema der Resonanz von DWG beschäftigt haben (s. u.), ist die Vielfalt an Begrifflichkeiten entsprechend groß: Ein sehr bezeichnender und zudem verbreiteter Begriff ist der der resonanten Wellenleitergitter - aus dem Englischen Resonant waveguide grating, kurz RWG (FANG u. a. 2006). Die darüber hinaus verwendeten Begriffe Guided-mode resonance (GMR, s. WANG u. a. 1990) bzw. Waveguide-mode resonance sowie die abgewandelte Form Guided-mode grating resonance GMGR, s. BAO und HuANG 2004, 2005) können knapp als »Moden-Resonanz « übersetzt werden und heben den resonanten Charakter der DWG oder die Anregung von Schwingungsmoden hervor. Insbesondere tauchen in der Literatur die anwendungsorientierten Begriffe Guided-mode resonance filter GMRF, s. MAGNUSSON und WANG 1992) sowie Resonant grating reflection filter (JACOB u. a. 2000) auf, welche klar die Filtereigenschaft dieser Strukturen betonen. 


\subsection{Resonante Wellenleitergitter in der Wissenschaft}

Die angegebenen Referenzen im Abschnitt zuvor machen deutlich, dass die Resonanz von DWG bereits seit vielen Jahrzehnten wissenschaftlich untersucht wird. Bis zur Entdeckung der Resonanzanomalien durch GolubENKO u. a. 1985 sind bereits zuvor viele wichtige Grundlagen für das Verständnis dieser Strukturen geschaffen worden. Durch Aufzeichnungen nachweisbare wissenschaftliche Untersuchungen zu Anomalien an optischen Beugungsgittern beginnen vor gut 100 Jahren: 1902 veröffentlicht ROBERT W. WOOD eine Arbeit, in der er seltsame Beugungsphänomene an feinmaschigen, metallischen Drahtgittern beschreibt, deren Effekt stark von der Polarisationsrichtung des einfallenden Lichts abhängt. Die von ihm beobachteten hellen und dunklen Banden werden in der Wissenschaft als WooDsche Anomalien bezeichnet und ziehen - mit dem Drang sie aufzuklären viele, zum Teil namhafte Wissenschaftler in ihren Bann: Unter anderem befassen sich der britische Physiker RAYLEIGH (1907) sowie der Göttinger Wissenschaftler VOIGT (1911) mit diesem Phänomen und schaffen die Grundlagen für eine theoretische Beschreibung. Im Jahre 1942 verfasst der Physiker KURT ARTMANN eine Arbeit über die anomale Reflexion von optischen Strichgittern, dessen Inhalt den damaligen Stand zu den theoretischen Arbeiten zusammenfassend wiedergibt.

Einen wissenschaftlichen Wendepunkt zu den theoretischen Arbeiten auf diesem Gebiet markiert der Beitrag von Hessel und OlineR 1965, in dem zum ersten Mal zwischen Beugungsanomalie nach RAYLEIGH, welche auf das Erscheinen bzw. Verschwinden von propagierenden Beugungsordnungen zurückzuführen ist, und Anomalien, die durch eine resonante Anregung der metallischen Struktur selbst auftreten, unterschieden wird. So können auch die bis dato durch die Beschichtung von metallischen Gittern hervorgerufenen Anomalien geklärt werden (HUTLEY und BIRD 1973). Mithilfe neuentwickelter holographischer Methoden ist es jedoch Mitte der 70er Jahre des 20. Jahrhunderts möglich periodische Strukturen in dielektrischen Schichten zu erzeugen, wodurch erstmals vollständig dielektrische Gitterstrukturen möglich werden.

Mitte der 80er Jahre des 20. Jahrhunderts untersuchen zwei wissenschaftliche Gruppen unabhängig voneinander die Beugungsphänomene an zum Teil mehrfach beschichteten dielektrischen Gittern: Zunächst zeigen MASHEV und POPOV 1984 
durch spektrale sowie winkelabhängige (angulare) Messungen, dass in Abhängigkeit der Polarisation ein anomales Resonanzverhalten der -1 . Beugungsordnung (LiTTRow-Anordnung) wahrnehmbar ist. Nahezu zeitgleich untersuchen GOLUBENKO u. a. (1985) das Resonanzverhalten an einfach-beschichteten dielektrischen Gittern und beobachten sowohl in Reflexion als auch in Transmission ein stark alternierendes Intensitätsverhalten der 0. Ordnung. Die rein phänomenologische Beschreibung der Anomalie wird auch von numerischen Berechnungen begleitet, mit deren Hilfe das Resonanzverhalten vorhersagbar wird. In der Veröffentlichung von MASHEV und POPOV 1985 wird das Resonanzverhalten zum ersten Mal winkelabhängig bei einer festen Laserwellenlänge von $632,816 \mathrm{~nm}$ sowie spektral mithilfe eines durchstimmbaren Farbstofflasers bei einem Resonanzwinkel von etwa 34,8 vermessen; die dabei gemessenen Reflektivitäten betragen etwa $35 \%$. In einer gemeinsamen Publikation werden die theoretischen Überlegungen beider Gruppen vereinigt und sogar erweitert (GOLUBENKO u. a. 1986).

Die Forschergruppe um M. G. Moharam veröffentlicht Anfang der 90er Jahre des 20. Jahrhunderts weitergehende theoretische Betrachtungen auf der Grundlage einer sog. rigorosen Beugungstheorie (engl. Rigorous coupled-wave theory), welche die Resonanz der DWG durch die Lösung von Eigenwertgleichungen beschreibt (WANG u. a. 1990). Rasch wird das Potential der DWG als polarisationssensitiver Filter oder als elektro-optisches Schaltelement in der integrierten Optik erkannt (Magnusson und Wang 1992; Wang und Magnusson 1993); die Patentanmeldung durch MAGNUSSON und WANG erfolgt 1993. Dabei gelingt es erstmalig Reflektivitäten von $94 \%$ für TM-polarisiertes Licht der Wellenlänge $1064 \mathrm{~nm}$ (MAGnusson u.a. 1998) und sogar von $98 \%$ bei einer Anregungswellenlänge von $860 \mathrm{~nm}$ experimentell nachzuweisen (LIU u. a. 1998). Zum besseren Verständnis der DWG.Resonanz wird zudem ein Interferenzmodell entwickelt, welches eine vergleichsweise anschauliche Beschreibung der Resonanz sowohl für die GWS JACOB u. a. 2000, 2001b) als auch für die DGWS (JACOB u. a. 2001a) erlaubt.

Die bereits erwähnte Gruppe um ASHER A. FRIESEM beschäftigt sich ebenfalls mit der theoretischen Beschreibung sowie der experimentellen Untersuchung an resonanten DWG (SHARON u. a. 1995). Jedoch konzentrieren sich die Aktivitäten zunehmend auf Strukturen, deren Filtereigenschaften auf der Basis von Halbleitermaterialien (SHARON u. a. 1996a) oder metallischen Schichten (SHARON u. a. 1997a) 
erreicht werden. Im Vordergrund stehen dabei besonders schmalbandige Transmissionsfilter (SHARON u.a. 1996b; LEVY-YURISTA und FRIESEM 2000, 2001) und deren Durchstimmbarkeit (GlasberG u. a. 1998; DUdovich u.a. 2001). In einer umfassenden Arbeit mit theoretischem und experimentellem Inhalt aus dem Jahr 1997 werden die extreme Schmalbandigkeit (ANGSTRÖM-Bereich) sowie die elektro-optischen Schalt- bzw. Modulationseigenschaften einer DWG auf Halbleiterbasis noch einmal betont (RosenblatT u. a. 1997). In der Konsequenz melden FRIESEM und SHARON 1999 Patent an, wobei die ursprüngliche Funktion dieser DWG-Form als aktiver Spiegel eines Laserresonators dabei stets weiterentwickelt und auf andere Forschungsgebiete (wie z. B. Biosensorik) ausgedehnt wird (FRIESEM u. a. 2002). Zeitgleich wird die Forschung an der bereits in früheren Arbeiten erwähnten DWG auf Polymerbasis ausgedehnt, so dass mit hybriden Strukturen aus halbleitenden und organischen Materialien Reflektivitäten bis $72 \%$ erreicht werden (DONVAL u. a. 2001; KATCHALSKI 2002).

Neben einer Anwendung als Resonatorspiegel in der Halbleiter-Lasertechnologie AvRUTSKY und RABADY 2001), wo ein Einsatz der DWG in einem modengekoppelten Halbleiterlaser mit Wellenlängendurchstimmbarkeit geprüft wird (WEBER und PAWLOWSKI 2000, PAWLOWSKI u. a. 2000), ist auch eine Verwendung in der Nachrichtentechnik als optischer Filter gegeben (STOCKERMANS 1998, WeBER u. a. 2004). Zunehmend wird auch eine Kombination mit nichtlinearen Merkmalen untersucht (NEvière u. a. 1995, 2000), bei der z. B. mithilfe eines nicht-linearen Polymerwellenleiters bei geeigneter intensiver Bestrahlung eine Änderung der Brechzahl erzeugt wird, wodurch wie bei den Halbleiterstrukturen eine Resonanzverschiebung (Durchstimmbarkeit) und/oder ein Schalteffekt möglich erscheint (BOYE u. a. 1999; BADER 2001, 2003).

Mit der Nutzung des Gitters zur Lichteinkopplung in den Wellenleiter (DAKSS u.a. 1970) werden die durch die Anregung der Schwingungsmoden auftretenden propagierenden und evaneszenten Wellen - auch am Koppelgitter (TIEFENTHALER und LUKOSZ 1989, KunZ u. a. 1996) - zur optischen Charakterisierung von chemischen oder biochemischen Stoffen zunehmend interessant, woraufhin in der Biosensorik innerhalb kurzer Zeit patentierte Verfahren zur Verfügung stehen (BUDACH und Neuschaefer 2001; DuvenECK u. a. 2001a). Wie sich zeigt, stellt die DWG bei resonanter Anregung im Vergleich zu konventionellen Methoden ein um zwei 
Größenordnungen erhöhtes evaneszentes Feld bereit, wodurch eine entsprechend hohe Sensitivität erreicht wird (NEUSCHÄFER u. a. 2003; BUDACH u. a. 2003).

\subsection{Motivation, Aufgabenstellung und Aufbau der Arbeit}

Motiviert durch die Reflektivitäten von bis zu $98 \%$ für den IR-Spektralbereich LIU u. a. 1998) wird angestrebt, vergleichbare Werte auch für die UV Wellenlängen $355 \mathrm{~nm}$ und $266 \mathrm{~nm}$ zu erhalten. Zwar existieren mit den etablierten dielektrischen Vielschichtsystemen bereits hochreflektierende Spiegel (BRAGG-Reflektoren) für den ultravioletten Spektralbereich, jedoch zeigen diese insbesondere im fernen UV. Bereich eine hohe Bandbreite von typischerweise $15 \mathrm{~nm}$ und nur selten eine Reflektivität von über $95 \%$ (EBERT und EBERT 2002). Mit der resonanten DWG als Reflektor mit vergleichsweise einfacher Struktur erscheint es möglich, mithilfe dielektrischer Metalloxidschichten, welche i. Allg. eine niedrige Absorption im ultravioletten Spektralbereich besitzen, eine hohe Reflektivität zu erzeugen.

Neben dem Erwerb kommerziell erhältlicher Substratgitter, welche i.d. R. nur in einer begrenzten Auswahl an Gitterperioden bzw. -tiefen vorliegen, scheint die Fertigung angepasster Submikrometer-Gitterstrukturen mithilfe der $\mathrm{F}_{2}$-Laserablation eine geeignete Methode zu sein (Ihlemann u. a. 2003). Mit dem Aufdampfen der wellenleitenden Schicht durch geeignete Verfahren, gelingt es, die Gitterstruktur im Substrat nahezu identisch an der Oberfläche der DWG zu reproduzieren. Diese Struktur in Form einer DGWS besitzt im Vergleich zur GWS in Abhängigkeit von der Phasenlage der Gitter untereinander eine höhere oder niedrigere spektrale Resonanzbandbreite (JACOB u. a. 2001a).

In Anlehnung an die DWG-Strukturen mit nicht-linear optischen bzw. elektrooptischen Komponenten (s. o.) soll das passive Strukturverhalten der DWG durch einen zusätzlichen Freiheitsgrad erweitert werden, um somit die bereits angesprochene Durchstimmbarkeit der Resonanz zu erhalten (LEITEL u. a. 2006). Dies soll mithilfe speziell gefertigter, poröser Wellenleiter geschehen, bei denen in Abhängigkeit von der Umgebungstemperatur der Brechungsindex der Schicht über die Einlagerung von Wasser veränderbar wird und damit die Resonanz der Struktur sowohl spektral als auch winkelabhängig (angular) durchstimmbar bleibt. 
Mit der DGWS als Sensorplattform innerhalb der Biotechnologie soll die Anwendung dieser resonanten Strukturen im Hinblick auf die sog. Zwei-PhotonenFluoreszenz untersucht werden. Wie bei der Anregung auf einem unstrukturierten, planaren Wellenleiter (DUVENECK u.a. 2001b) soll dabei geprüft werden, ob sich auf der strukturierten Oberfläche der DGWS wie bei der Ein-PhotonenFluoreszenzanregung (NEUSCHÄFER u. a. 2003) einzelne Moleküle oder Molekülschichten biologischer Substanzen durch die Bestrahlung mit ultrakurzen Laserpulsen anregen lassen. Der Schwerpunkt der Untersuchungen liegt hierbei zunächst auf dem Nachweis bestimmter Farbstoffmoleküle (Markerfarbstoffe) sowie der Bestimmung einer möglichen Fluoreszenzverstärkung durch die DGWS.

Einschließlich der Einleitung gliedert sich die Arbeit in sechs Kapitel: Die ersten vier Unterkapitel in Kapitel 2 schaffen aufeinander aufbauend die Grundlagen für eine theoretische, vorwiegend analytische Beschreibung des elektromagnetischen Resonanzphänomens bei dielektrischen Wellenleitergittern. Der letzte der fünf Abschnitte in Kapitel 2 widmet sich ausgewählter numerischer Berechnungen unter Berücksichtigung rigoroser Methoden. Kapitel 3 befasst sich mit der Herstellung der Strukturen, d. h. mit den Verfahren zur Fertigung des Gitters und des Wellenleiters, und erläutert dabei die Gitterstrukturierung mithilfe der $\mathrm{F}_{2}$-Laserablation genauer. Die beiden letzten Abschnitte der insgesamt fünf Unterkapitel widmen sich der Oberflächencharakterisierung erzeugter Proben bzw. der daraus gewonnenen Informationen zu den Strukturdaten. Mit der optischen Charakterisierung der DWG Proben befasst sich Kapitel 4, wobei ausführlich das angulare sowie spektrale Resonanzverhalten der Proben in Reflexion und Transmission bei der Bestrahlung mit unterschiedlichsten Wellenlängen untersucht wird. Unter anderem wird dabei die Durchstimmbarkeit einer DGWS-Resonanz bei Änderung der Umgebungstemperatur gezeigt und die zeitliche Pulslänge der Leckwelle mithilfe eines Autokorrelators bestimmt. Im Hinblick auf das Anwendungspotentials der DWG als Sensorplattform beschäftigt sich Kapitel 5 mit dem Nachweis von Fluoreszenzfarbstoffen durch Zwei-Photonen-Anregung. Insbesondere wird dabei ein Fluoreszenz-Verstärkungsfaktor ermittelt sowie der Nachweis eines Nonapeptids auf einer DGWS-Oberfläche in wässriger Umgebung erbracht. Eine Zusammenfassung mit einem kurzen Ausblick wird in Kapitel 6 gegeben. 


\section{Theorie zu Wellenleitergittern}

Dieses Kapitel widmet sich der theoretischen Beschreibung von dielektrischen Wellenleitergittern (DWG). Die hier gezeigten, hauptsächlich analytischen Formeln sollen zum besseren physikalischen Verständnis rund um das Resonanzphänomen der DWG beitragen. Einleitend geht Abschnitt 2.1 auf die Polarisierbarkeit bzw. die Totalreflexion elektromagnetischer Wellen ein und beschreibt damit eine ihrer wichtigsten Eigenschaften. Im Anschluss widmen sich Abschnitt 2.2 und 2.3 der Anregung von Schwingungsmoden bzw. der Beugung am Gitter, welche die Grundlage für die Beschreibung der elektromagnetischen Resonanz bilden. Darauf aufbauend trägt Abschnitt 2.4.1 die wichtigsten Formeln zur analytischen Beschreibung resonanter DWG zusammen und geht dabei speziell auf die Phasenanpassung, die Resonanzverschiebung durch Temperaturänderung sowie auf das Intensitätsverhalten in Transmission und Reflexion ein. Am Schluss des Kapitels werden in Abschnitt 2.5 ausgewählte Beispielrechnungen auf der Grundlage analytischer Formeln und rigoroser Methoden vorgestellt.

\subsection{Polarisierbarkeit und Totalreflexion elektromagnetischer Wellen}

Die Ausbreitung einer elektromagnetischen, monochromatischen Welle lässt sich durch die MAXWELLsche Theorie aus den Jahren 1861-64 beschreiben. Im Gegensatz zu älteren Fernwirkungstheorien ist diese eine Nahwirkungs- bzw. Feldtheorie und beschreibt die Erzeugung von elektrischen und magnetischen Feldern durch Ladungen und Ströme. Darüber hinaus werden auftretende Wechselwirkungen zwischen zeitabhängigen Feldern berücksichtigt (JACKSON|2006). Die aus der Theorie abgeleiteten sog. MAXWELLschen Gleichungen (1865) bilden innerhalb der Wellenoptik die Grundlage für eine mathematische Beschreibung von elektromagnetischen 
Wellen und sind daher in vielen Optik-Lehrbüchern zu finden (LAUTERBORN u.a. 1993, BORN und WOLF 1999).

Mithilfe der MAXWELL-Gleichungen lässt sich eine (homogene) Wellengleichung für sich eine im Vakuum ausbreitende Welle in Abhängigkeit der Funktion $\vec{E}(\vec{r}, t)$ aufstellen (HELmHoLtz-Gleichung):

$$
\frac{\partial^{2} \vec{E}(\vec{r}, t)}{\partial x^{2}}+\frac{\partial^{2} \vec{E}(\vec{r}, t)}{\partial y^{2}}+\frac{\partial^{2} \vec{E}(\vec{r}, t)}{\partial z^{2}}=\frac{1}{c_{0}^{2}} \cdot \frac{\partial^{2} \vec{E}(\vec{r}, t)}{\partial t^{2}}
$$

Die elektrische Feldstärke $\vec{E}(\vec{r}, t)$ ist dabei sowohl raum- [Ortsvektor $\vec{r}=(x, y, z)$ ] als auch zeitabhängig (Variable $t$ ). Die konstante Größe $c_{0}$ bezeichnet die Vakuumlichtgeschwindigkeit, welche über die Gleichung $c_{0}=1 / \sqrt{\varepsilon_{0} \mu_{0}}$ mit der elektrischen Leitfähigkeit $\varepsilon_{0}$ (Permittivität) und magnetischen Leitfähigkeit $\mu_{0}$ (Permeabilität) verknüpft ist. Eine Lösung der Gleichung (2.1) ist u. a. die harmonische Welle, welche mithilfe der Kreisfrequenz $\omega$ (Zeitkomponente) und dem Wellenvektor $\vec{k}$ (Richtungskomponente) in der Form

$$
\vec{E}(\vec{r}, t)=E_{0} \cdot \mathrm{e}^{\mathrm{i}\left(\vec{k}_{0} \cdot \vec{r}-\omega_{0} \cdot t\right)}
$$

dargestellt werden kann. Der Index 0 markiert hierbei eine Ausbreitung der Welle im Vakuum. Eine weitere Lösung von (2.1) ist mit der zugehörigen magnetischen Feldstärke $\vec{H}(\vec{r}, t)$ gegeben, welche analog zu 2.2 durch

$$
\vec{H}(\vec{r}, t)=H_{0} \cdot \mathrm{e}^{\mathrm{i}\left(\vec{k}_{0} \cdot \vec{r}-\omega_{0} \cdot t\right)}
$$

gegeben ist. Die komplexen Amplituden beider Felder sind über die Beziehung

$$
\sqrt{\mu_{0}} \cdot H_{0}=\sqrt{\varepsilon_{0}} \cdot E_{0}
$$

miteinander verknüpft (BORN und WOLF 1999). Zudem zeigt die Relation 


$$
\vec{H}=\sqrt{\frac{\mu_{0}}{\varepsilon_{0}}} \cdot \frac{\vec{k}}{|\vec{k}|} \times \vec{E}
$$

dass die Vektorfelder im freien Raum senkrecht aufeinander stehen. Die Welle mit der Ausbreitungsrichtung $\vec{k}$ wird daher auch als Transversal- oder Querwelle bezeichnet, d.h. die Schwingungsrichtung der elektromagnetischen Feldvektoren $\vec{E}$ und $\vec{H}$ sind senkrecht zur Ausbreitungsrichtung $\vec{k}$ orientiert. Der Betrag des Wellenvektors $\vec{k}$ - auch Kreiswellenzahl genannt - verhält sich dabei umgekehrt proportional zur Wellenlänge $\lambda$ :

$$
|\vec{k}| \equiv k=\frac{\omega}{c}=\frac{2 \cdot \pi}{\lambda}
$$

Bei der Betrachtung von elektromagnetischen Wellen in Medien kann die Permittivität eines Materials als Produkt aus der Vakuum-Permittivität $\varepsilon_{0}$ und der materialspezifischen relativen Permittivität $\varepsilon_{r}$ dargestellt werden:

$$
\varepsilon=\varepsilon_{0} \cdot \varepsilon_{r}
$$

Analog dazu lässt sich der Einfluss auf das magnetische Feld durch die Permeabilität des Materials ausdrücken:

$$
\mu=\mu_{0} \cdot \mu_{r} .
$$

Die relative Permittivität und Permeabilität lassen sich unter Berücksichtigung der MAXWELL-Gleichungen zu einer gemeinsamen optischen Brechzahl (Brechungsindex) $n$ zusammenfassen (BORN und WOLF 1999):

$$
n=\sqrt{\varepsilon_{r} \mu_{r}} .
$$


Diese ist i. d. R. größer als die Brechzahl $n_{0}$ im Vakuum und hat zur Folge, dass sich die Ausbreitungsgeschwindigkeit (Phasengeschwindigkeit) c der Welle innerhalb des Mediums ändert:

$$
\mathrm{c}=\frac{\mathrm{c}_{0}}{n}=\frac{1}{\sqrt{\varepsilon \mu}}=\frac{1}{\sqrt{\varepsilon_{0} \mu_{0} \varepsilon_{r} \mu_{r}}} .
$$

Der Brechungsindex besitzt jedoch in Abhängigkeit vom durchlaufenden Medium auch eine Komponente $\kappa$, welche die imaginäre Einheit i mit $\dot{i}^{2}=-1$ als Faktor trägt. Die vollständige Brechzahl $n$ lässt sich somit als komplexe Zahl $\tilde{n}$ schreiben:

$$
\tilde{n}=n+\mathrm{i} \kappa
$$

mit $\tilde{n} \in \mathbb{C}, n=\operatorname{Re}\{\tilde{n}\}$ und $\kappa=\operatorname{Im}\{\tilde{n}\}$. Mit dem Einsetzen der komplexen Brechzahl $\tilde{n}$ in die Wellengleichung (2.1) (unter Berücksichtigung von 2.10 und (2.11) ) wird die Ausbreitung der Welle für $\kappa>0$ gedämpft. Daher wird $\kappa$ auch als sog. Extinktionskoeffizient bezeichnet. Über die Frequenz $\nu$ der Welle bzw. die Wellenlänge $\lambda$ ist $\kappa$ zudem mit dem sog. Absorptionskoeffizienten $\alpha$ verknüpft

$$
\kappa=\frac{\alpha \cdot \mathrm{c}}{4 \pi \cdot \nu}=\frac{\alpha \cdot \lambda}{4 \pi}
$$

welcher bei der Absorption von Strahlung eine etablierte Größe darstellt (LAMBERT-BEERsches-Gesetz).

Um eine Aussage über die räumliche Schwingungsrichtung (Polarisation) einer elektromagnetischen Welle zu erhalten, können die Feldvektoren $\vec{E}$ und $\vec{H}$ in ihre Richtungskomponenten zerlegt werden:

$$
\vec{E}=\left(\begin{array}{c}
E_{x} \\
E_{y} \\
E_{z}
\end{array}\right) \text { und } \vec{H}=\left(\begin{array}{c}
H_{x} \\
H_{y} \\
H_{z}
\end{array}\right)
$$


TM-Polarisation

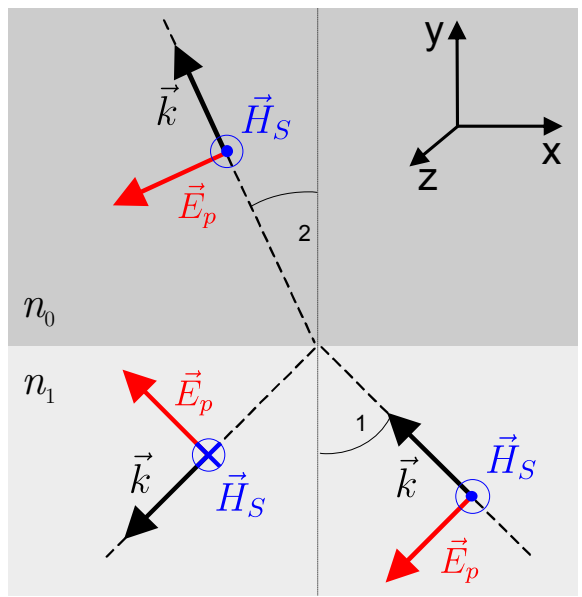

- Vektor zeigt aus Ebene heraus
TE-Polarisation

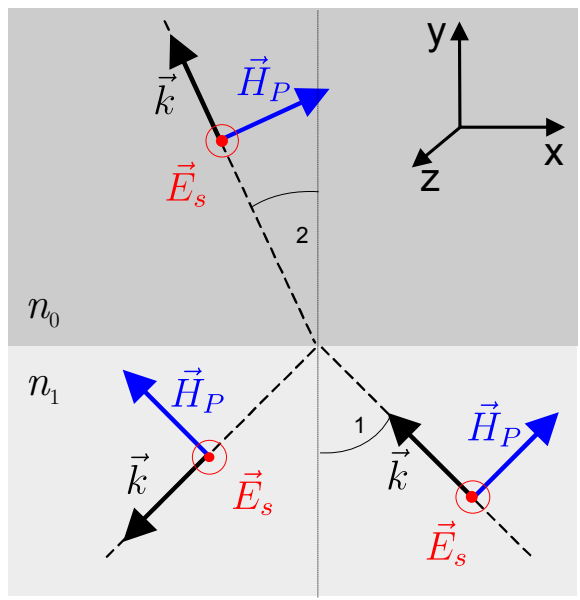

$\boldsymbol{\otimes}$ Vektor zeigt in Ebene hinein

Abb. 2.1: Konvention für die positive Richtung von $\vec{k}, \vec{E}$ und $\vec{H}$ beim Medienübergang $n_{1}$ nach $n_{0}\left(n_{0}>n_{1}\right)$ im Fall von TM. bzw. TE Polarisation.

Bei linearer Polarisation, d.h. die räumliche Schwingungsrichtung bleibt zeitlich konstant, lässt sich das (kartesische) Koordinatensystem so legen, dass einer der Feldvektoren $(\vec{E}$ bzw. $\vec{H})$ in einer Achse des Koordinatensystems mit den Basisvektoren $\left\{\vec{e}_{x}, \vec{e}_{y}, \vec{e}_{z}\right\}$ liegt. Für den Fall, dass das $\vec{E}$ - bzw. $\vec{H}$-Feld parallel zur x-y-Ebene liegt, spricht man von $p$ - bzw. P-Polarisation. Steht der E- bzw. H-Feldvektor senkrecht zur Ebene, d.h. er liegt in $z$-Richtung, ist die $s$ - bzw. $S$-Polarisation gemeint:

$$
\begin{gathered}
\vec{E}_{p}=E_{x} \cdot \vec{e}_{x}+E_{y} \cdot \vec{e}_{y} \text { und } \vec{E}_{s}=E_{z} \cdot \vec{e}_{z} \\
\vec{H}_{P}=H_{x} \cdot \vec{e}_{x}+H_{y} \cdot \vec{e}_{y} \text { und } \vec{H}_{S}=H_{z} \cdot \vec{e}_{z}
\end{gathered}
$$

Wie man anhand von Gleichung 2.5 sieht, stehen die $\vec{E}$ - und $\vec{H}$-Felder dabei stets senkrecht aufeinander. So besitzt die Welle für $\vec{E}_{p}$ nur eine $H$-Komponente in $z$-Richtung, nämlich $H_{z}$, die senkrecht zur Ausbreitungsebene (x-y-Ebene) liegt. Diese Feldausrichtung wird auch transversal-magnetische (kurz TM) Polarisation 
genannt. Analog besitzt die Welle für $\vec{E}_{s}$ zwei $H$-Komponenten in der x-y-Ebene $H_{x}$ bzw. $H_{y}$, was eine transversal-elektrische - kurzTE- Ausrichtung beschreibt.

In Abb. 2.1 sind alle Feld- und Wellenvektoren beim Medienübergang von $n_{1}$ nach $n_{0}$ mit $n_{0}>n_{1}$ für beide Polarisationen dargestellt. Die sog. transversalelektromagnetische (TEM) Welle bildet einen Spezialfall, bei der keinerlei Feldkomponenten in Ausbreitungsrichtung liegen.

Das geometrische (makroskopische) Verhalten eines gerichteten Lichtstrahls an der Grenzfläche zweier benachbarter Medien lässt sich durch das SNELLIUSsche Brechungsgesetz beschreiben. Unter der Annahme ebener Wellen, d.h. $\vec{k}_{0} \cdot \vec{r}=$ const, zeigt das Gesetz die Winkeländerung des transmittierten Strahls in Abhängigkeit der Brechungsindizes $n_{1}$ und $n_{0}$ :

$$
n_{1} \cdot \sin \theta_{1}=n_{0} \cdot \sin \theta_{0}
$$

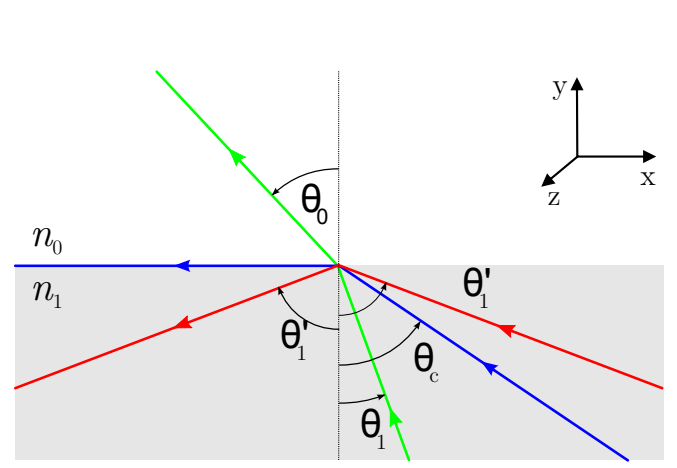

Abb. 2.2: Skizze zu 2.16 mit $n_{1}>$ $n_{2}$ für verschiedene Einfallswinkel $\theta_{1}^{\prime}>$ $\theta_{\mathrm{c}}>\theta_{1}$. Ab $\theta_{\mathrm{c}}$ tritt Totalreflexion auf. wobei $\theta_{1}$ und $\theta_{2}$ den Ein- bzw. Ausfallswinkel der Lichtstrahlen darstellen (BORN und WoLF 1999). Verläuft der Strahl vom optisch dichteren $n_{1}$ zum optisch dünneren Medium $n_{0}$ wird der zunehmende Einfallswinkel irgendwann kritisch (Abb. 2.2). Für den Fall, dass $\theta_{0}$ senkrecht zum Lot, also im Winkel $90^{\circ}$ steht (blauer Strahl), spricht man vom Grenzwinkel der Totalreflexion bzw. vom kritischen Winkel $\theta_{c}$. Dieser ist für $n_{1}>n_{0}$ durch die Formel

$$
\theta_{c}=\sin ^{-1}\left(\frac{n_{0}}{n_{1}}\right) \equiv \sin ^{-1} n_{01}
$$

angegeben. Danach erfährt eine einfallende Welle mit dem Winkel $\theta_{1} \stackrel{\text { def }}{=} \theta_{1}^{\prime}>\theta_{c}$ eine Totalreflexion (roter Strahl); engl. Total internal reflection - kurz TIR. 
An der Grenzfläche innerhalb des optisch dünneren Mediums $n_{0}$ wird aufgrund der TIR eine schnell abklingende Welle, ein sog. evaneszentes Feld, erzeugt AXELROD u.a. 1992). Die Existenz dieser Felder lässt sich durch konventionelle bzw. geometrische Betrachtung nicht mehr erklären, sondern folgt aus den Lösungen der MAXWELLGleichungen (DE FORNEL 2001). Der

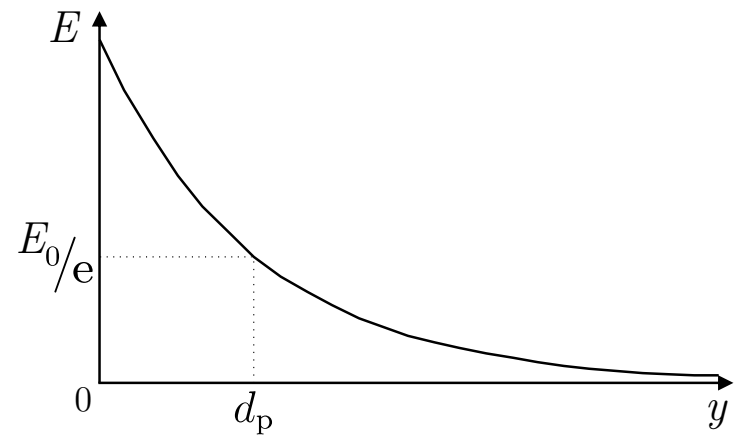

Abb. 2.3: Exponentieller Abfall des evaneszenten $\vec{E}$-Feldes an der Grenzfläche $(y=0)$. Betrag der komplexen Amplitude $E_{0}$ bzw. $H_{0}$ fällt hinter der Grenzfläche mit zunehmendem Abstand exponentiell ab, d. h. die Amplitudenverteilung in $z$-Richtung ist beispielsweise für das $E$-Feld durch $|\vec{E}(\vec{r})|=E \sim E_{0} \cdot \exp \left(-y / d_{\mathrm{p}}\right)$ gegeben (s. Abb2.3). Die Eindringtiefe $d_{\mathrm{p}}$, bei der das Feld auf den 1/e-Anteil abgefallen ist, wird dabei u. a. vom (überkritischen) Einfallswinkel $\theta_{1}$ bestimmt:

$$
d_{\mathrm{p}}=\frac{\lambda}{2 \pi \sqrt{n_{1}^{2} \cdot \sin ^{2} \theta_{1}-n_{0}^{2}}} .
$$

Die Streuung von evaneszenten Wellen (engl. Evanescent wave scattering, kurz EWS) an Oberflächen oder Nanopartikeln findet zudem Anwendung beim sog. optischen Rasternahfeldmikroskop - engl. Scanning nearfield optical microscope, kurz SNOM (NOVOTNY und HECHT 2006).

Gleichzeitig wird mit dem Auftreten von TIR eine Verschiebung der totalreflektierten Welle beobachtet, welche erstmals von GOOS und HÄNCHEN (1947) nachgewiesen wird. Dieser sog. GoOs-HÄNCHEN-Effekt ist durch einen longitudinalen Versatz $L_{x}$ gekennzeichnet, welcher in Abhängigkeit von der (linearen) Polarisationsrichtung (TE bzw. TM) zu unterschiedlichen Verschiebungen führt \begin{tabular}{|l|l|l|l|}
\hline GOOS und LINDBERG-HÄNCHEN & 1949 & IMBERT und LEVY & 1975) \\
\hline
\end{tabular}

$$
L_{x}^{\overline{T M}} \sim\left|\overrightarrow{E_{p}}\right|^{2} \text { bzw. } L_{x}^{\sqrt{\mathrm{TE}}} \sim\left|\overrightarrow{E_{s}}\right|^{2} .
$$


Durch TIR beeinflusste zirkular- bzw. elliptisch polarisierte Wellen zeigen zudem einen transversalen Versatz $L_{z}$, welcher als sog. IMBERT. FEDOROV.Effekt bekannt ist (IMBERT und LEVY 1975):

$$
L_{z} \sim \vec{E}_{p} \cdot \vec{E}_{s}^{*}-\vec{E}_{s} \cdot \vec{E}_{p}^{*}
$$

Diese laterale Verschiebung, welche von FEDOROV (1955) vorausgesagt und später von IMBERT untersucht worden ist, fällt i.d. R. bis zu einer Größenordnung kleiner aus als die GoOs-HÄNCHEN-Verschiebung (LOTSCH 1968). Ausführliche bzw. weiterführende Informationen zu TIR Verschiebungen sind in der Arbeit von LOTSCH (1970, 1971) zu finden.

Die Länge $L_{\mathrm{GH}}$ der longitudinalen GoOs-HÄNCHEN-Verschiebung wird durch die Änderung der Phasenverschiebung $\mathrm{d} \Phi / \mathrm{d} \theta_{1}$ der einfallenden Welle bestimmt ARTMANN 1948):

$$
L_{\mathrm{GH}} \stackrel{\text { def }}{=} L_{x}=\frac{\lambda}{2 \pi \cdot \cos \theta_{1}} \cdot\left(\frac{\mathrm{d} \Phi}{\mathrm{d} \theta_{1}}\right)
$$

Da die Phasenverschiebung $\Phi$ - wie 2.19 bereits andeutet - von der Polarisation der Welle abhängt, ergeben sich für die TE bzw. TM.Polarisation unterschiedliche TIR.Phasensprünge $\Phi_{\text {TE }}$ bzw. $\Phi_{\overline{T M}}$ (BORN und WOLF 1999):

$$
\begin{gathered}
\Phi_{\overline{\mathrm{TE}}}=-2 \cdot \tan ^{-1}\left(\frac{\sqrt{\sin ^{2} \theta_{1}-n_{01}^{2}}}{\cos \theta_{1}}\right), \\
\Phi_{\overline{\mathrm{TM}}}=-2 \cdot \tan ^{-1}\left(\frac{\sqrt{\sin ^{2} \theta_{1}-n_{01}^{2}}}{n_{01}^{2} \cdot \cos \theta_{1}}\right) .
\end{gathered}
$$

Die Gleichungen 2.22) und 2.23 werden u. a. zur analytischen Beschreibung der Phase im Wellenleiter bei der Anregung von Schwingungsmoden benötigt. 


\subsection{Schwingungsmoden in planaren Wellenleitern}

Die Anregung von stehenden Wellen innerhalb von planaren Wellenleitern, sog. transversale Schwingungsmoden, lässt sich analytisch über die Phasenverschiebung des inneren bezüglich des äußeren Feldes beschreiben.

Abb. 2.4 zeigt das geometrische Modell (Zick-Zack-Verlauf) zur Ausbreitung einer Schwingungsmode im Wellenleiter (Brechzahl $n_{1}$ ). An den Grenzflächen zu den niedrig brechenden Medien $n_{0}$ und $n_{2}$ mit $\max \left\{n_{0}, n_{2}\right\}<n_{1}$ tritt bedingt durch TIR die in Formel 2.21 propagierte GoosHÄNCHEN-Verschiebung $L_{\mathrm{GH}}$ in Abhängigkeit von $n_{0}$ und $n_{2}$ auf. Analog zu 2.22 und 2.23 lassen sich zum jeweiligen Grenzübergang die polarisationsabhängigen Phasensprünge $\Phi \frac{T \Phi}{10}$ und $\Phi \frac{T \Phi}{12}$ bzw. Verschiebung $L_{\mathrm{GH}}$ von $k_{1}$ auf. $\Phi \frac{T M}{10}$ und $\Phi \frac{T M}{12}$ berechnen.

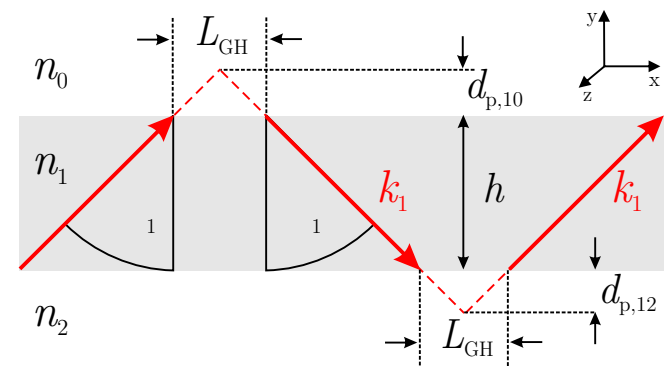

Abb. 2.4: Geometrisches Modell zur Ausbreitung einer Schwingungsmode im Wellenleiter der Brechzahl $n_{1}$. Aufgrund von TIR tritt an den Grenzflächen eine

Der Weg- bzw. Gangunterschied $G$, den die Welle nach einem vollständigen Umlauf im Wellenleiter zurücklegt, lässt sich über die Wellenleiterdicke $h$ und den Winkel $\theta$ beschreiben (BORN und WOLF 1999; HunsPERGER 2002):

$$
G=2 h \cdot n_{1} \cdot \cos \theta_{1}
$$

Die dabei auftretende (dimensionslose) Phasenverschiebung $\Phi_{G}$ kann mithilfe der Kreiswellenzahl $k_{1}$ (s. Abb. 2.4) dargestellt werden:

$$
\Phi_{G}=k_{0} \cdot G \equiv \frac{4 \pi}{\lambda_{0}} \cdot n_{1} \cdot h \cdot \cos \theta_{1}=2 \cdot k_{1} \cdot h \cdot \cos \theta_{1}
$$

Um die Gesamtphasenverschiebung $\Phi_{W L}$ der Welle $\mathrm{zu}$ erhalten, genügt es, die einzelnen Phasen aufzuaddieren: 


$$
\begin{aligned}
& \Phi \frac{\mathrm{TE}}{\mathrm{WL}}=\Phi_{G}+\Phi \frac{\mathrm{TE}}{10}+\Phi \frac{T \mathrm{TE}}{12}, \\
& \Phi \frac{\mathrm{TM}}{\mathrm{WL}}=\Phi_{G}+\Phi \frac{\overline{\mathrm{TM}}}{10}+\Phi \frac{\overline{T M}}{12} .
\end{aligned}
$$

Die vollständige Darstellung von $\Phi \frac{T \mathrm{TE}}{\mathrm{WL}}$ und $\Phi \frac{\mathrm{TM}}{\mathrm{WL}}$ lautet demnach:

$$
\begin{array}{r}
\Phi \frac{\mathrm{TE}}{\mathrm{WL}}=\frac{4 \pi \cdot h \cdot n_{1} \cdot \cos \theta_{1}}{\lambda_{0}}-2 \cdot \tan ^{-1}\left(\frac{\sqrt{\sin ^{2} \theta_{1}-n_{01}^{2}}}{\cos \theta_{1}}\right) \\
-2 \cdot \tan ^{-1}\left(\frac{\sqrt{\sin ^{2} \theta_{1}-n_{21}^{2}}}{\cos \theta_{1}}\right), \\
\Phi \frac{\mathrm{TM}}{\mathrm{WL}}=\frac{4 \pi \cdot h \cdot n_{1} \cdot \cos \theta_{1}}{\lambda_{0}}-2 \cdot \tan ^{-1}\left(\frac{\sqrt{\sin ^{2} \theta_{1}-n_{01}^{2}}}{n_{01}^{2} \cdot \cos \theta_{1}}\right) \\
-2 \cdot \tan ^{-1}\left(\frac{\sqrt{\sin ^{2} \theta_{1}-n_{21}^{2}}}{n_{21}^{2} \cdot \cos \theta_{1}}\right) .
\end{array}
$$

Damit die Anregung einer Schwingungsmode erfolgen kann, muss die Bedingung zur Phasenanpassung (engl. Phase matching condition) im wellenleitenden Medium erfüllt sein (HUNSPERGER 2002):

$$
\Phi_{\mathrm{WL}}=M \cdot 2 \pi
$$

Die Modenzahl $M$ gibt hierbei die Anzahl der Schwingungsmoden an, welche für monochromatisches Licht der Wellenlänge $\lambda_{0}$ mithilfe der Wellenleiterdicke $h$ sowie den Brechzahlen $n_{0}$ und $n_{2}$ mit $n_{2} \geq n_{0}$ berechnet werden kann (MARCUSE 1991): 


$$
\begin{aligned}
& M^{\mathrm{TE}}=\frac{1}{\pi} \cdot\left[\frac{2 \pi \cdot h}{\lambda_{0}} \sqrt{n_{1}^{2}-n_{2}^{2}}-\tan ^{-1}\left(\frac{\sqrt{n_{2}^{2}-n_{0}^{2}}}{\sqrt{n_{1}^{2}-n_{2}^{2}}}\right)\right], \\
& M^{\overline{\mathrm{TM}}}=\frac{1}{\pi} \cdot\left[\frac{2 \pi \cdot h}{\lambda_{0}} \sqrt{n_{1}^{2}-n_{2}^{2}}-\tan ^{-1}\left(\frac{n_{1}^{2}}{n_{0}^{2}} \cdot \frac{\sqrt{n_{2}^{2}-n_{0}^{2}}}{\sqrt{n_{1}^{2}-n_{2}^{2}}}\right)\right] .
\end{aligned}
$$

Die um die Eindringtiefen $d_{\mathrm{p}, 10}$ und $d_{\mathrm{p}, 12}$ verbreiterte Wellenleiterdicke $h$ (vgl. Abb. 2.4 wird auch als effektive Wellenleiterdicke $h_{\text {eff }}$ bezeichnet:

$$
h_{\mathrm{eff}}=h+d_{\mathrm{p}, 10}+d_{\mathrm{p}, 12}
$$

Die Ausbreitung der geführten Welle ist durch die sog. Propagationskonstante $\beta$ bestimmt, welche die in $z$-Richtung abgeleitete (effektive) Kreiswellenzahl $k_{1}$ angibt:

$$
\beta=k_{1} \cdot \sin \theta_{1} \equiv n_{1} \cdot k_{0} \cdot \sin \theta_{1}
$$

Der Quotient aus $\beta$ und $k_{0}$ wird als effektiver Modenindex $N$ bezeichnet:

$$
N=\beta / k_{0}=n_{1} \cdot \sin \theta_{1} .
$$

Für $N$ bzw. $\beta$ gilt stets

$$
n_{1}>N>\max \left\{n_{2}, n_{0}\right\} \text { bzw. } k_{1}>\beta>k_{0} \cdot \max \left\{n_{2}, n_{0}\right\}
$$

wobei $\beta$, wie man anhand der Gleichung 2.28) und 2.29) durch Substitution von 2.34 erkennen kann, selbst auch von der Polarisation abhängt: $\beta_{\overline{\mathrm{TE}}} \neq \beta_{\overline{\mathrm{TM}}}$. 
Nach Abb. 2.1 sowie den Formeln (2.14) und 2.15) reduzieren sich die Feldanteile von $\vec{E}$ und $\vec{H}$ bei TE-Polarisation auf $H_{x}, H_{y}$ und $E_{z}$, bzw. auf $E_{x}, E_{y}$ und $H_{z}$ bei TM-Polarisation. Wie für die Wellengleichung im Vakuum [vgl. (2.1)] lässt sich mithilfe der MAXWELLschen-Gleichungen für die elektrische Feldkomponente $E_{z}$ die sog. Helmholtz-Gleichung für die Wellenleitung einer TE-polarisierten Welle aufstellen (MARCUSE 1991):

$$
\frac{\partial^{2} \vec{E}_{z}}{\partial^{2} x^{2}}+\left(n^{2} \cdot k^{2}-\beta_{\text {TE }}^{2}\right) \cdot E_{z}=0
$$

Analog verteilt sich die $H_{z}$-Komponente der TM-Schwingungsmode wie folgt:

$$
\frac{\partial^{2} \vec{H}_{z}}{\partial^{2} x^{2}}+\left(n^{2} \cdot k^{2}-\beta_{\mathrm{TM}}^{2}\right) \cdot H_{z}=0
$$

Die ausführliche Herleitung der Wellengleichungen sowie die vollständige Darstellung aller Feldkomponenten kann in verschiedenen Monographien nachgeschlagen werden (KOGELNIK 1979, 1990; MARCUSE 1991).

\subsection{Dielektrisches Beugungsgitter}

Bei den im Rahmen dieser Arbeit verwendeten optischen Beugungsgittern, handelt es sich um sog. Reliefgitter, welche eine rein passive Gitterstruktur mit einem spezifischen Profil (Relief) auf der Oberfläche einer dielektrischen Schicht aufweisen. Für das Reliefgitter typische Profile sind das Rechteckprofil (Laminargitter) und das Sinusprofil (Sinusgitter). Das Beugungsverhalten im Fernfeld (FrAunhofERBeugung) einer unter dem Winkel $\theta_{0}$ einfallenden ebenen Welle $\left(\vec{k}_{0} \cdot \vec{r}=\right.$ const $)$ lässt sich über die sog. Gittergleichung beschreiben (LOEWEN und POPOV 1997). Für die unter dem Winkel $\theta_{0, m}$ gebeugte Welle lautet die Formel in Reflexion

$$
n_{0} \cdot \sin \theta_{0, m}=n_{0} \cdot \sin \theta_{0}+m \cdot \frac{\lambda_{0}}{\Lambda}
$$


bzw. in Transmission:

$$
n_{1} \cdot \sin \theta_{1, m}=n_{0} \cdot \sin \theta_{0}+m \cdot \frac{\lambda_{0}}{\Lambda}
$$

wobei $\Lambda$ die Gitterperiode und $m$ die Beugungsordnung mit $m \in \mathbb{Z}$ angibt.

Die in Abb. 2.5 gezeigten Gitterkonstanten $a$ und $b$ geben mit $a+b=\Lambda$ die Ausdehnung der Brechungsindexbereiche für $n_{0}$ bzw. $n_{1}$ an. Das Verhältnis von $b$ und $\Lambda$ wird als Tastverhältnis oder Füllfaktor bezeichnet. Da es sich bei den Reliefgittern (Laminargitter in Abb. 2.5 - wie in 2.39 und 2.40 angedeutet - um eindimensionale, räumlich kohärente Elemente handelt, deren Beugungseigenschaften nur durch $\Lambda$ bestimmt sind, lässt sich die Beugung einer einfallenden Welle der Richtung $\vec{k}_{0} \stackrel{\text { def }}{=} \vec{k}=\left\{k_{x}, k_{y}, 0\right\}$

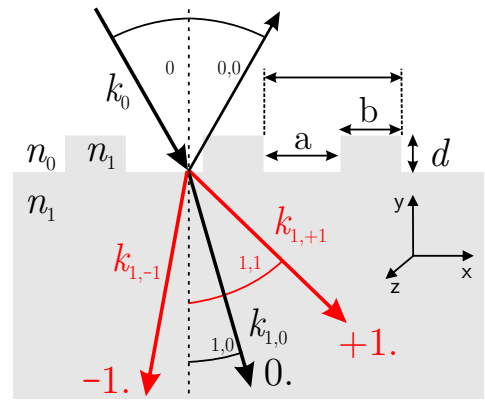

Abb. 2.5: Geometrisches Modell zur Gitterbeugung. mithilfe des reziproken Gittervektors $\vec{K}$ mit $|\vec{K}| \equiv$ $K=2 \pi / \Lambda$ beschreiben. Liegt $\vec{K}$ wie in Abb. 2.5 in $x$-Richtung kann das Beugungsverhalten des Gitters in Transmission über die $x$-Komponenten der Vektorbeträge mit $|\vec{K}|=K_{x}$ ausgedrückt werden:

$$
n_{1} \cdot k_{x, m} \stackrel{\text { def }}{=} k_{1, x, m}=n_{0} \cdot k_{x}+m \cdot K_{x}
$$

Der Vektor $\vec{k}_{1, m}$ mit den Komponenten $\left\{k_{1, x, m}, k_{1, y, m}, 0\right\}$ gibt den Wellenvektor im Medium $n_{1}$ mit $n_{1}>n_{0}$ an. Wird die Komponente $k_{y, m}$ mit $k_{y, m}=\sqrt{k^{2}+k_{x, m}^{2}}$ innerhalb des jeweiligen Mediums imaginär, d.h. $k_{y, m} \in \mathbb{C} \backslash \mathbb{R}$, spricht man auch von evaneszenten Beugungsordnungen (NEVIÈRE und POPOV 2003). Im Vergleich zu den Ordnungen mit $k_{y, m} \in \mathbb{R}$ bilden die evaneszenten Ordnungen jedoch keine propagierenden Wellen aus, d.h. im Fernfeld werden keine Beugungsordnungen sichtbar.

Wellenlängenabhängige Erscheinungen, welche auf die Evaneszenz, d.h. das Verschwinden von propagierten Beugungsordnungen, oder auf die Anregung von 
(polarisationsabhängigen) Resonanzen zurückgeführt werden können, werden als sog. WOODsche Anomalien bezeichnet (WOOD 1902). Die speziell auf der Evaneszenz beruhende, polarisationsunabhängige Erscheinungsform wird auch RAYLEIGH-Anomalie genannt (RAYleigh 1907; Hessel und OlineR 1965). Diese Form der Anomalie ereignet sich bei $\theta_{1, m}=90^{\circ}$, so dass für den Einfallswinkel $\theta_{0}$ nach Umstellung von 2.39 gilt:

$$
\theta_{0}=\sin ^{-1}\left(1 \mp m \cdot \frac{\lambda_{0}}{\Lambda}\right)
$$

Analog zu 2.40 gilt für die Wood-RAYLEIGH-Anomalie in Transmission:

$$
\theta_{0}=\sin ^{-1}\left(\frac{n_{2}}{n_{0}} \mp \frac{m}{n_{0}} \cdot \frac{\lambda_{0}}{\Lambda}\right)
$$

Aufgrund der Gittertiefe $d$, wobei i.d. R. $d<\lambda_{0}$ ist, wird das dielektrische Beugungsgitter auch als Phasengitter bezeichnet, welches im Unterschied zu einem Amplitudengitter eine Phasenverschiebung im Fernfeld erzeugt. Die Verschiebung der Phase im Vergleich zur 0. Beugungsordnung $(m=0)$ steigt dabei mit der Ordnungszahl $m$ um den Wert $\pi / 2$ an (SOMMERFELD 1964).

\subsection{Analytische Beschreibung resonanter Wellenleitergitter}

Aufbauend auf die vorangegangenen Abschnitte über die Wellenleitung und Gitterbeugung ist es möglich, eine vollständig analytische Beschreibung der Gesamtphase von dielektrischen Wellenleitergittern (DWG) unter Resonanzbedingungen zu geben. Zur Veranschaulichung liefert Abb. 2.6 anhand einer Gitter-WellenleiterStruktur GWS, vgl. 3.1 ein geometrisches Modell zur Ein- bzw. Auskopplung der am Gitter gebeugten Teilstrahlen $(m=1)$ einer einfallenden Welle der Kreiswellenzahl $k_{0}$. Während die 0. Beugungsordnung innerhalb der hochbrechenden Wellenleiterschicht $\left(n_{1}>\max \left\{n_{0}, n_{2}\right\}\right)$ mit einem Beugungswinkels $\theta_{1,0}$ keine Totalreflexion an der Grenzfläche zum Medium $n_{2}$ erfährt, ist der Winkel $\theta_{1,1}$ der 
+1. Beugungsordnung groß genug, den Teilstrahl $k_{1,+1}$ vollständig zu reflektieren. Wird dieser nach einem Umlauf erneut an der Grenzfläche zwischen $n_{0}$ und $n_{1}$ total-reflektiert, kann durch die Phasenanpassung der einfallenden (äußeren) mit der gebeugten (inneren) Welle eine polarisationsabhängige Schwingungsmode angeregt werden. Die An-

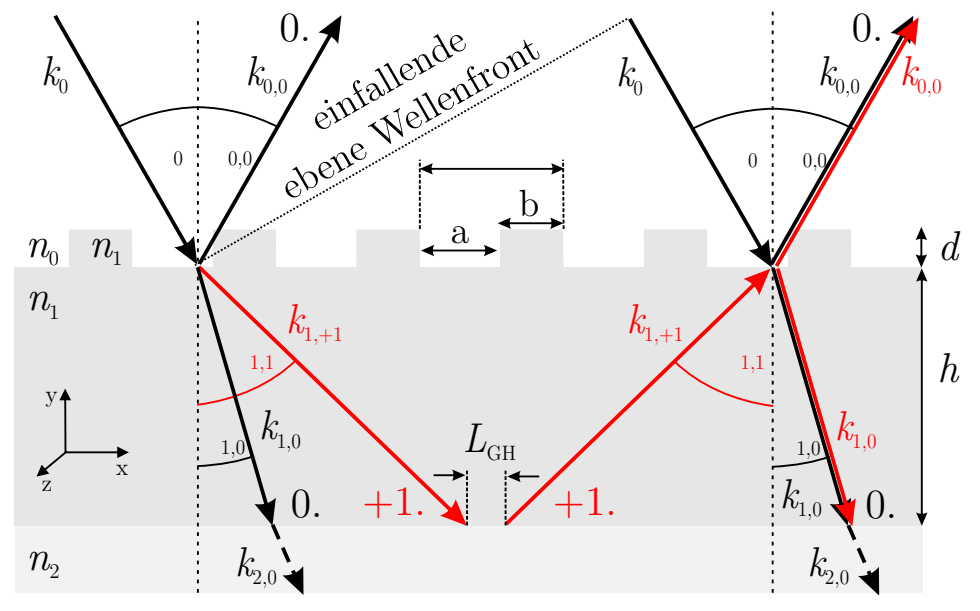

Abb. 2.6: Geometrisches Modell zur resonanten Ein- bzw. Auskopplung der +1 . Beugungsordnung $(m=1)$ eines dielektrischen Wellenleitergitters (DWG mit einer Gitterstruktur oberhalb des Wellenleiters (GWS). wesenheit des (ideal unendlich ausgedehnten) Gitters verhindert jedoch die Ausbreitung der Mode, so dass sich eine sog. dissipative Leckwelle (engl. Leaky waveguide mode) ausbildet (NEVIERE u.a. 1977), welche - eine hinreichende Gitterlänge vorausgesetzt die vollständige Auskopplung der geführten Welle erzwingt GOLUBENKO u.a. 1985). Die sog. Kopplungslänge der Resonanz $l_{\mathrm{R}}$, welche die charakteristische Länge für die Aufenthaltszeit der geführten Mode ist, hängt dabei invers von der Dissipationskonstante $\gamma$ ab (s. u.).

Bedingt durch die zweifache Diffraktion (Ein- und Auskopplung) ist die wiederausgekoppelte Leckwelle unter Resonanzbedingungen um insgesamt $\pi$ zur einfallenden bzw. transmittierten Welle verschoben. In der Folge findet eine destruktive Interferenz (Auslöschung) zwischen der Leckwelle $k_{1,0}$ und der direkt unter der 0 . Beugungsordnung transmittierten Welle $k_{1,0}$ statt, so dass in Resonanz (idealerweise) keine Transmission beobachtet wird $\left(k_{2,0} \rightarrow 0\right)$. Anders verhält sich die Leckwelle in Reflexion: Aufgrund eines Phasensprungs von $\pi$ der direkt reflektierten Welle 0 . Ordnung sind $k_{0,0}$ und $k_{0,0}$ gleichphasig, d. h. sie überlagern konstruktiv. Da es sich hierbei jedoch um eine rein analytische Betrachtung der Resonanz handelt, kommt die exakte Beschreibung dieses Phänomens, d.h. die vollständi- 
ge Lösung der MAXWELLschen Gleichungen durch numerische Rechenverfahren (s. Abschnitt 2.5), nicht immer zum selben Ergebnis.

Für eine möglichst hohe Reflektivität bei Anregung der DWG durch die \pm 1 . Beugungsordnung erhalten, ist es sinnvoll, das Auftreten von WoOD-RAYLEIGHAnomalien sowie die Erzeugung höherer Beugungsordnungen zu vermeiden. Mithilfe der Gleichungen 2.42 und 2.43 lassen sich die entsprechenden Randbedingungen ableiten: Für eine reflektierte Welle 0. Ordnung $(|m|<1)$ lässt die Gitterperiode $\Lambda$ in Abhängigkeit von $\lambda_{0}$ und $\theta_{0}$ durch folgende Ungleichung nach oben abschätzen:

$$
\Lambda<\frac{\lambda_{0}}{n_{0}+n_{0} \cdot\left|\sin \theta_{0}\right|}
$$

Unter Berücksichtigung, dass auch in Transmission keine höhere Ordnungen auftreten sollen, muss $n_{0}$ in 2.44 durch die Bedingung $\max \left\{n_{0}, n_{2}\right\}$ ersetzt werden. Um einer Evaneszenz der propagierten Beugungsordnung für $|m|=1$ zu entgehen, ist $\Lambda$ durch die Bedingung $\sin \theta_{1,1}<1$ auch nach unten begrenzt. Die vollständige Abschätzung für $m= \pm 1$ lautet demnach (STENZEL 2003, 2005):

$$
\frac{\lambda_{0}}{n_{1} \mp n_{0} \sin \theta_{0}}<\Lambda<\frac{\lambda_{0}}{\max \left\{n_{0}, n_{2}\right\} \mp n_{0} \cdot \sin \theta_{0}}
$$

Analog zu Ungleichung 2.45 lässt sich bei feststehenden Strukturparametern $\Lambda$, $n_{0}$ und $n_{1}$ die obere und untere Grenze die Anregungswellenlänge $\lambda_{0}$ in Abhängigkeit von $\theta_{0}$ darstellen:

$$
\left(\max \left\{n_{0}, n_{2}\right\} \mp n_{0} \cdot \sin \theta_{0}\right) \cdot \Lambda<\lambda_{0}\left(\theta_{0}\right)<\left(n_{1} \mp n_{0} \cdot \sin \theta_{0}\right) \cdot \Lambda
$$

Der winkelabhängige Wellenlängenbereich, welcher durch die obere und untere Grenze in 2.46) dargestellt ist, kennzeichnet den sog. Resonanzkorridor SELLE $2003)$, d. h. den Bereich, in welchem eine Totalreflexion der \pm 1 . Beugungsordnung 
zur Anregung einer Schwingungsmode stattfinden kann. Der an den Korridor angrenzende Raum evaneszenter Ordnungen wird auch als »Verbotener Bereich « bezeichnet (s. Abb. 2.7). Die Breite des Korridors ist für einen festen Einfallswinkel $\theta_{0}$ durch $\left(n_{1}-\max \left\{n_{0}, n_{2}\right\}\right) \cdot \Lambda$ bestimmt. Da $n_{0}$ und $n_{2}$ von $\lambda_{0}$ abhängen können (Dispersion), kann die Breite des Resonanzkorridors für verschiedene Winkel variieren.

\subsubsection{Phasenanpassung resonanter Wellenleitergitter}

Betrachtet man die Phase der in Abb. 2.6 zur Anregung einer Schwingungsmode gezeigte +1 . Beugungsordnung genauer, so muss festgestellt werden, dass neben dem Phasenversatz $\pi$ / 2 (Beugung) auch ein FRESNEL-Versatz beim Medienübergang (Brechung) stattfindet. Der Betrag dieser Verschiebung ist gerade $L_{\mathrm{GH}} / 2$ (RosenblatT u. a. 1997), so dass der gebeugte Teilstrahl $k_{1,+1}$ im Vergleich zur einfallenden Welle nach einem vollständigen Umlauf im Wellenleiter einen Gesamtphasenversatz von 2.28 bzw. 2.29 besitzt. Wie man beim Vergleich der Abb. 2.4 und 2.6 sieht, entspricht der Modenwinkel $\theta_{1}$ dem Beugungswinkel $\theta_{1, m}$, so dass mit $\theta_{1, m} \stackrel{\text { def }}{=} \theta_{1}$ eine Kopplung der Formeln 2.28 und 2.29 mit der Gittergleichung 2.40 gelingt. Bei Vernachlässigung der Gittertiefe $d$ lauten die vollständigen Phasenanpassungsgleichungen für eine durch einen Teilstrahl der Beugungsordnung $m$ angeregten Mode $M$ (SELLE 2003; STENZEL 2005):

$$
\begin{aligned}
\lim _{d \rightarrow 0} \Phi \frac{\text { TE }}{\text { DWG }} & =\frac{4 \pi \cdot h}{\lambda_{0}} \cdot \sqrt{n_{1}^{2}-\left(n_{0} \cdot \sin \theta_{0}+m \cdot \frac{\lambda_{0}}{\Lambda}\right)^{2}} \\
& -2 \cdot \tan ^{-1}\left(\frac{\sqrt{\left(n_{0} \cdot \sin \theta_{0}+m \cdot \lambda_{0} / \Lambda\right)^{2}-n_{0}^{2}}}{\sqrt{n_{1}^{2}-\left(n_{0} \cdot \sin \theta_{0}+m \cdot \lambda_{0} / \Lambda\right)^{2}}}\right) \\
& -2 \cdot \tan ^{-1}\left(\frac{\sqrt{\left(n_{0} \cdot \sin \theta_{0}+m \cdot \lambda_{0} / \Lambda\right)^{2}-n_{2}^{2}}}{\sqrt{n_{1}^{2}-\left(n_{0} \cdot \sin \theta_{0}+m \cdot \lambda_{0} / \Lambda\right)^{2}}}\right) \\
& \equiv M \cdot 2 \pi,
\end{aligned}
$$




$$
\begin{aligned}
\lim _{d \rightarrow 0} \Phi \frac{\text { TM }}{\mathrm{DWG}} & =\frac{4 \pi \cdot h}{\lambda_{0}} \cdot \sqrt{n_{1}^{2}-\left(n_{0} \cdot \sin \theta_{0}+m \cdot \frac{\lambda_{0}}{\Lambda}\right)^{2}} \\
& -2 \cdot \tan ^{-1}\left(\frac{n_{1}^{2}}{n_{0}^{2}} \cdot \frac{\sqrt{\left(n_{0} \cdot \sin \theta_{0}+m \cdot \lambda_{0} / \Lambda\right)^{2}-n_{0}^{2}}}{\sqrt{n_{1}^{2}-\left(n_{0} \cdot \sin \theta_{0}+m \cdot \lambda_{0} / \Lambda\right)^{2}}}\right) \\
& -2 \cdot \tan ^{-1}\left(\frac{n_{1}^{2}}{n_{2}^{2}} \cdot \frac{\sqrt{\left(n_{0} \cdot \sin \theta_{0}+m \cdot \lambda_{0} / \Lambda\right)^{2}-n_{2}^{2}}}{\sqrt{n_{1}^{2}-\left(n_{0} \cdot \sin \theta_{0}+m \cdot \lambda_{0} / \Lambda\right)^{2}}}\right) \\
& \equiv M \cdot 2 \pi .
\end{aligned}
$$

Die Darstellung des Einfallswinkels $\theta_{0}$ über der Wellenlänge $\lambda_{0}$ wird als Resonanzkennlinie bezeichnet (s. Abb. 2.7). Da die Gleichungen (2.47) und (2.48) jedoch die Gittertiefe $d$ nicht berücksichtigen, gelten die Gleichungen nur für Wellenleitergitter, bei denen die Gittertiefe vernachlässigt werden kann $(\Lambda \gg d)$. Mit der Bildung eines mittleren (reellwertigen) Brechungsindex $\bar{n}_{10}$, welcher in der Theorie von RYTOV (1956) zur Berechnung einer effektiven Brechzahl an periodischen Medien (engl. Effective medium theory, kurz EMT) beschrieben wird, ist es dennoch möglich, die Gitterstruktur als homogene Schicht mit in die analytische Betrachtung einfließen zu lassen. In einer Näherung der EMT mit der Annahme $\lambda_{0} \gg \Lambda$ lautet die Brechzahl $\bar{n}_{10}$ für die TE. bzw. TM-Mode (KunZ u. a. 1996):

$$
\begin{aligned}
& \bar{n}_{10 \text { TE }}^{2}=\frac{a}{\Lambda} \cdot n_{0}^{2}+\frac{b}{\Lambda} \cdot n_{1}^{2}, \\
& \frac{1}{\bar{n}_{10 \text { TM }}^{2}}=\frac{a}{\Lambda} \cdot \frac{1}{n_{0}^{2}}+\frac{b}{\Lambda} \cdot \frac{1}{n_{1}^{2}} .
\end{aligned}
$$

Mit der von JACOB u.a. (2000) vorgestellten Anpassung der Phasenverschiebung unter Berücksichtigung der Gittertiefe $d$ lassen sich die Formeln (2.22) und 2.23 für den Phasensprung an der jeweiligen Grenzfläche durch einen zusätzli- 
chen Verschiebungsfaktor (Tangens-Hyperbolicus-Funktion) entsprechend anpassen (ADAMS 1981):

$$
\begin{aligned}
& \bar{\Phi} \overline{10}=-2 \cdot \tan ^{-1}\left\{\frac{\sqrt{\left(n_{0} \cdot \sin \theta_{0}+m \cdot \lambda_{0} / \Lambda\right)^{2}-\bar{n}_{10}^{2}}}{\sqrt{n_{1}^{2}-\left(n_{0} \cdot \sin \theta_{0}+m \cdot \lambda_{0} / \Lambda\right)^{2}}}\right. \\
& \cdot \tanh \left[\tanh ^{-1}\left(\frac{\sqrt{\left(n_{0} \cdot \sin \theta_{0}+m \cdot \lambda_{0} / \Lambda\right)^{2}-n_{0}^{2}}}{\sqrt{\left(n_{0} \cdot \sin \theta_{0}+m \cdot \lambda_{0} / \Lambda\right)^{2}-\bar{n}_{10 \mathrm{TE}}^{2}}}\right)\right. \\
& \left.\left.+\frac{2 \pi \cdot d}{\lambda_{0}} \cdot \sqrt{\left(n_{0} \cdot \sin \theta_{0}+m \cdot \lambda_{0} / \Lambda\right)^{2}-\bar{n}_{10 \mathrm{TE}}^{2}}\right]\right\} \text {, } \\
& \bar{\Phi} \overline{10}=-2 \cdot \tan ^{-1}\left\{\frac{n_{1}^{2}}{\bar{n}_{10 \mathrm{TM}}^{2}} \cdot \frac{\sqrt{\left(n_{0} \cdot \sin \theta_{0}+m \cdot \lambda_{0} / \Lambda\right)^{2}-\bar{n}_{10}^{2} \mathrm{TM}}}{\sqrt{n_{1}^{2}-\left(n_{0} \cdot \sin \theta_{0}+m \cdot \lambda_{0} / \Lambda\right)^{2}}}\right. \\
& \cdot \tanh \left[\tanh ^{-1}\left(\frac{\bar{n}_{10}^{2}}{n_{0}^{2}} \cdot \frac{\sqrt{\left(n_{0} \cdot \sin \theta_{0}+m \cdot \lambda_{0} / \Lambda\right)^{2}-n_{0}^{2}}}{\sqrt{\left(n_{0} \cdot \sin \theta_{0}+m \cdot \lambda_{0} / \Lambda\right)^{2}-\bar{n}_{10}^{2}}}\right)\right. \\
& \left.\left.+\frac{2 \pi \cdot d}{\lambda_{0}} \cdot \sqrt{\left(n_{0} \cdot \sin \theta_{0}+m \cdot \lambda_{0} / \Lambda\right)^{2}-\bar{n}_{10[\mathrm{TM}}^{2}}\right]\right\} \text {. }
\end{aligned}
$$

Bei der Betrachtung einer Doppel-Gitter-Wellenleiter-Struktur (DGWS, vgl. Abb. 1.1) muss zudem zusätzlich eine Gitterstruktur zwischen Wellenleiter und Substrat berücksichtigt werden: Die Formeln für $\bar{\Phi} \frac{T \Phi}{12}$ und $\bar{\Phi} \frac{T M}{12}$ werden analog zu 2.51 bzw. 2.52 angepasst. Für eine DGWS mit identischer Gittertiefe $d$ oberhalb und unterhalb des Wellenleiters lauten die vollständigen Phasenanpassungsgleichungen: 


$$
\begin{aligned}
& \Phi \frac{\mathrm{TE}}{\mathrm{DGWS}}=\Phi_{G}+\bar{\Phi} \frac{\mathrm{TE}}{10}+\Phi_{\frac{\mathrm{TE}}{12}}^{\mathrm{TE}} \\
& =\frac{4 \pi \cdot h}{\lambda_{0}} \cdot \sqrt{n_{1}^{2}-\left(n_{0} \cdot \sin \theta_{0}+m \cdot \frac{\lambda_{0}}{\Lambda}\right)^{2}} \\
& -2 \cdot \tan ^{-1}\left\{\frac{\sqrt{\left(n_{0} \cdot \sin \theta_{0}+m \cdot \lambda_{0} / \Lambda\right)^{2}-\bar{n}_{10}^{2} \mathrm{TE}}}{\sqrt{n_{1}^{2}-\left(n_{0} \cdot \sin \theta_{0}+m \cdot \lambda_{0} / \Lambda\right)^{2}}}\right. \\
& \cdot \tanh \left[\tanh ^{-1}\left(\frac{\sqrt{\left(n_{0} \cdot \sin \theta_{0}+m \cdot \lambda_{0} / \Lambda\right)^{2}-n_{0}^{2}}}{\sqrt{\left(n_{0} \cdot \sin \theta_{0}+m \cdot \lambda_{0} / \Lambda\right)^{2}-\bar{n}_{10 \text { TE }}^{2}}}\right)\right. \\
& \left.\left.+\frac{2 \pi \cdot d}{\lambda_{0}} \cdot \sqrt{\left(n_{0} \cdot \sin \theta_{0}+m \cdot \lambda_{0} / \Lambda\right)^{2}-\bar{n}_{10 \mathrm{TE}}^{2}}\right]\right\} \\
& -2 \cdot \tan ^{-1}\left\{\frac{\sqrt{\left(n_{0} \cdot \sin \theta_{0}+m \cdot \lambda_{0} / \Lambda\right)^{2}-\bar{n}_{12}^{2} \mathrm{TE}}}{\sqrt{n_{1}^{2}-\left(n_{0} \cdot \sin \theta_{0}+m \cdot \lambda_{0} / \Lambda\right)^{2}}}\right. \\
& \cdot \tanh \left[\tanh ^{-1}\left(\frac{\sqrt{\left(n_{0} \cdot \sin \theta_{0}+m \cdot \lambda_{0} / \Lambda\right)^{2}-n_{2}^{2}}}{\sqrt{\left(n_{0} \cdot \sin \theta_{0}+m \cdot \lambda_{0} / \Lambda\right)^{2}-\bar{n}_{12 \mathrm{TE}}^{2}}}\right)\right. \\
& \left.\left.+\frac{2 \pi \cdot d}{\lambda_{0}} \cdot \sqrt{\left(n_{0} \cdot \sin \theta_{0}+m \cdot \lambda_{0} / \Lambda\right)^{2}-\bar{n}_{12 \mathrm{TE}}^{2}}\right]\right\} \\
& \equiv M \cdot 2 \pi,
\end{aligned}
$$




$$
\begin{aligned}
& \Phi \frac{\overline{\mathrm{TM}}}{\mathrm{DGWS}}=\Phi_{G}+\Phi_{\frac{\mathrm{TM}}{10}}+\bar{\Phi} \frac{\overline{T M}}{12} \\
& =\frac{4 \pi \cdot h}{\lambda_{0}} \cdot \sqrt{n_{1}^{2}-\left(n_{0} \cdot \sin \theta_{0}+m \cdot \frac{\lambda_{0}}{\Lambda}\right)^{2}} \\
& -2 \cdot \tan ^{-1}\left\{\frac{n_{1}^{2}}{\bar{n}_{10 \text { TM }}^{2}} \cdot \frac{\sqrt{\left(n_{0} \cdot \sin \theta_{0}+m \cdot \lambda_{0} / \Lambda\right)^{2}-\bar{n}_{10}^{2} \text { TM }}}{\sqrt{n_{1}^{2}-\left(n_{0} \cdot \sin \theta_{0}+m \cdot \lambda_{0} / \Lambda\right)^{2}}}\right. \\
& \cdot \tanh \left[\tanh ^{-1}\left(\frac{\bar{n}_{10[\mathrm{TM}}^{2}}{n_{0}^{2}} \cdot \frac{\sqrt{\left(n_{0} \cdot \sin \theta_{0}+m \cdot \lambda_{0} / \Lambda\right)^{2}-n_{0}^{2}}}{\sqrt{\left(n_{0} \cdot \sin \theta_{0}+m \cdot \lambda_{0} / \Lambda\right)^{2}-\bar{n}_{10 \text { TM }}^{2}}}\right)\right. \\
& \left.\left.+\frac{2 \pi \cdot d}{\lambda_{0}} \cdot \sqrt{\left(n_{0} \cdot \sin \theta_{0}+m \cdot \lambda_{0} / \Lambda\right)^{2}-\bar{n}_{10 \mathrm{TM}}^{2}}\right]\right\} \\
& -2 \cdot \tan ^{-1}\left\{\frac{n_{1}^{2}}{\bar{n}_{12 \mathrm{TM}}^{2}} \cdot \frac{\sqrt{\left(n_{0} \cdot \sin \theta_{0}+m \cdot \lambda_{0} / \Lambda\right)^{2}-\bar{n}_{12 \mathrm{TM}}^{2}}}{\sqrt{n_{1}^{2}-\left(n_{0} \cdot \sin \theta_{0}+m \cdot \lambda_{0} / \Lambda\right)^{2}}}\right. \\
& \cdot \tanh \left[\tanh ^{-1}\left(\frac{\bar{n}_{12 \text { TM }}^{2}}{n_{0}^{2}} \cdot \frac{\sqrt{\left(n_{0} \cdot \sin \theta_{0}+m \cdot \lambda_{0} / \Lambda\right)^{2}-n_{2}^{2}}}{\sqrt{\left(n_{0} \cdot \sin \theta_{0}+m \cdot \lambda_{0} / \Lambda\right)^{2}-\bar{n}_{12 \mathrm{TM}}^{2}}}\right)\right. \\
& \left.\left.+\frac{2 \pi \cdot d}{\lambda_{0}} \cdot \sqrt{\left(n_{0} \cdot \sin \theta_{0}+m \cdot \lambda_{0} / \Lambda\right)^{2}-\bar{n}_{12 \mathrm{TM}}^{2}}\right]\right\} \\
& \equiv M \cdot 2 \pi,
\end{aligned}
$$

Die mittleren Brechzahlen $\bar{n}_{10 \text { TE }}^{2}$ und $\bar{n}_{10 \text { TM }}^{2}$ (oberes Gitter) bzw. $\bar{n}_{12 \text { TE }}^{2}$ und $\bar{n}_{12 \text { TM }}^{2}$ (unteres Gitter) werden nach den Formeln 2.49) und 2.50 berechnet. Demnach lässt sich die Resonanz einer DWG (hier DGWS mithilfe der Gleichungen 2.53) und (2.54) in Abhängigkeit der Strukturparameter $\Lambda, a, h, d, m, M$ und $n_{i}(i=$ $0,1,2)$ sowie von den optischen Eigenschaften der einfallenden Welle $\theta_{0}$ und $\lambda_{0}$ vollständig analytisch beschreiben.

Aufgrund der kurzen mittleren Verweildauer resonant koppelnder Photonen ist die optische Weglänge des Lichts jedoch kürzer als bei der klassischen Wellen- 
leitung. Zusätzlich zur Propagationskonstante $\beta$ [vgl. (2.34)] muss demnach ein Dämpfungsterm $\gamma$ (Dissipationskonstante) berücksichtigt werden, welcher ein Maß für den Verlust der propagierten Mode durch die dissipative Leckwelle darstellt. Die komplexe Darstellung der Propagationskonstante lautet daher (NORTON u.a. 1997):

$$
\bar{\beta}=\beta+\mathrm{i} \gamma
$$

Mithilfe der Dissipationskonstante $\gamma$ kann der Leckwelle eine sog. Kopplungslänge der Resonanz $l_{\mathrm{R}}$ zugeordnet werden

$$
l_{\mathrm{R}}=\gamma^{-1}
$$

welche typischerweise im Sub-Millimeterbereich liegt. Wie die Arbeit von NORTON (1997) zur sog. Theorie der gekoppelten Moden (engl. Coupled-mode theory, kurz CMT zeigt, lässt sich mithilfe von $\gamma$ bzw. $l_{\mathrm{R}}$ die spektrale Resonanzbandbreite $\Delta \lambda_{\text {FWHM }}$ in ihrer Halbwertsbreite (engl. Full width at half maximum, kurz FWHM) bestimmen:

$$
\Delta \lambda_{\overline{\text { FWHM }}}=\frac{\lambda_{0} \cdot \Lambda}{\pi} \cdot \gamma \equiv \frac{\lambda_{0} \cdot \Lambda}{\pi} \cdot \frac{1}{l_{\mathrm{R}}}
$$

Darüber hinaus lässt sich $\Delta \lambda_{\text {FWHM }}$ durch die Ableitung $\mathrm{d} \theta_{0} / \mathrm{d} \lambda_{0}=m /\left(\Lambda \cdot \cos \theta_{\mathrm{R}}\right)$ aus Gleichung 2.39 in die Resonanzwinkel-(Halbwerts-)Breite $\Delta \theta_{\text {FwHM }}[\mathrm{rad}]$ mit $\theta_{\mathrm{R}}$ als Resonanzwinkel umformen:

$$
\Delta \theta_{\mathrm{FWHM}}=\frac{m \cdot \lambda_{0}}{\pi \cdot \cos \theta_{\mathrm{R}}} \cdot \gamma \equiv \frac{m \cdot \lambda_{0}}{\pi \cdot \cos \theta_{\mathrm{R}}} \cdot \frac{1}{l_{\mathrm{R}}}
$$

$\mathrm{Zu}$ den Gleichungen 2.57) und 2.58 sei noch erwähnt: Bereits 1989 gelingt es AVRUTSKY u. a. durch phänomenologische Betrachtungen nahezu identische Formeln für die Resonanzbandbreite bzw. die Resonanzwinkelbreite $\Delta \theta$ einer DGWS 
(AvRutsky u. a. 1989) sowie einer GWS (AvRUTSKY und SyCHugOV 1989) aufzustellen.

Aus der Kombination von 2.57) und 2.58) kann der spektralen eine angulare Resonanzbreite zugeordnet werden:

$$
\Delta \lambda_{\overline{\text { FWHM }}}=\frac{\Lambda \cdot \cos \left(\theta_{\mathrm{R}}\right)}{m} \cdot \Delta \theta_{\overline{\mathrm{FWHM}}} \cdot
$$

Wie im nachfolgenden Abschnitt gezeigt wird, lässt sich die angulare bzw. spektrale Halbwertsbreite auch über die Phasenverschiebung $\Phi_{\overline{\mathrm{DWG}}}$ ausdrücken.

\subsubsection{Infinitesimale Betrachtung der Phasenverschiebung}

Die durch die Gleichungen 2.53) und 2.54 gezeigte Interdependenz der Gesamtphase einer DWG unter Resonanzbedingungen $\Phi_{\overline{\mathrm{DWG}}}$ von 11 freien Parametern

$$
\Phi_{\overline{\mathrm{DWG}}}\left(\theta_{0}, \lambda_{0}, \Lambda, a, h, d, n_{0}, n_{1}, n_{3}, m\right) \equiv 2 \pi \cdot M
$$

bildet die Grundlage für die analytische Betrachtung der Resonanz bei der Variation einzelner oder mehrerer Parameter. Die infinitesimale Betrachtung von $\Phi_{\overline{\mathrm{DWG}}}$ erlaubt es, das Intensitätsverhalten (vgl. 2.4.3) bei Änderung von $\lambda_{0}$ und $\theta_{0} \mathrm{zu}$ analysieren. Mithilfe von $\Phi_{\overline{\text { FWHM }}}$, welches die Phasenlage kennzeichnet, bei der die Intensität der einfallenden Welle $I_{0}$ ein Einbruch um die Hälfte erfährt, kann das folgende totale Differential aufgestellt werden:

$$
\Delta \Phi_{\overline{\mathrm{FWHM}}}=\frac{\partial \Phi_{\overline{\mathrm{DWG}}}}{\partial \theta_{0}} \cdot \Delta \theta_{\overline{\mathrm{FWHM}}}+\frac{\partial \Phi_{\overline{\mathrm{DWG}}}}{\partial \lambda_{0}} \cdot \Delta \lambda_{0}+\cdots=0 .
$$

Mit der aus der Analogie zum FPI abgeleiteten Phase $\Phi$ FWHM (JACOB u. a. 2000) mit

$$
\Phi_{\overline{\mathrm{FWHM}}}=4 \cdot \sin ^{-1}\left(\frac{\eta}{2 \cdot \sqrt{1-\eta}}\right) \approx \frac{2 \eta}{\sqrt{1-\eta}}
$$


lassen sich die Halbwertsbreiten der DWG für den jeweiligen Parameter aufstellen:

$$
\begin{aligned}
& \Delta \theta_{\overline{\text { FWHM }}} \approx \frac{2 \eta}{\sqrt{1-\eta}} \cdot\left(\frac{\partial \Phi_{\overline{\mathrm{DWG}}}}{\partial \theta_{0}}\right)^{-1}, \\
& \Delta \lambda_{\overline{\mathrm{FWHM}}} \approx \frac{2 \eta}{\sqrt{1-\eta}} \cdot\left(\frac{\partial \Phi_{\overline{\mathrm{DWG}}}}{\partial \lambda_{0}}\right)^{-1} .
\end{aligned}
$$

Bedingt durch die doppelte Gitterstruktur setzt sich der Diffraktionskoeffizient für eine DGWS $\hat{\eta}$ aus den Koeffizienten $\eta_{10}$ (oberes Gitter) und $\eta_{12}$ (unteres Gitter) zusammen (JACOB u. a. 2001a):

$$
\hat{\eta}=\eta_{10}+\eta_{12}+2 \sqrt{\eta_{10} \cdot \eta_{12}} \cdot \sin \delta
$$

Der Phasenversatz $\delta$ ist durch die Laufstrecke der Welle von einem Gitter zum anderen bestimmt:

$$
\delta=\frac{2 \pi \cdot h \cdot \sqrt{n_{1}^{2}-\sin ^{2} \theta_{0}}}{\lambda_{0}}
$$

In erster Näherung lassen sich die Koeffizienten $\eta_{10}$ und $\eta_{12}$ für die \pm 1 . Beugungsordnung wie folgt darstellen (SHARON u. a. 1997b; NORTON u. a. 1998):

$$
\begin{aligned}
& \eta_{10} \approx\left[\frac{\left(n_{1}^{2}-n_{0}^{2}\right) \cdot k_{0}^{2} \cdot d}{2 K}\right]^{2}, \\
& \eta_{12} \approx\left[\frac{\left(n_{1}^{2}-n_{2}^{2}\right) \cdot k_{0}^{2} \cdot d}{2 K}\right]^{2} .
\end{aligned}
$$

Wie Berechnungen zur angularen und spektralen Resonanzbreite nach 2.63 bzw. (2.64) für die Näherung $\hat{\eta} \approx \eta_{10}+\eta_{12}$ gezeigt haben SELLE 2003; KAPPEL u. a. 2004b), ist eine deutlich breitere spektrale bzw. angulare Resonanz der DGWS 
im Vergleich zur GWS zu erkennen. Unter Berücksichtigung des Phasenversatzes $\delta$ variiert die spektrale Breite der DGWS im Vergleich zur GWS sogar um den Faktor 0 bis 4 (JACOB u. a. 2001b).

Wie man anhand der Relationen (2.67) bzw. (2.68) erkennen kann, lassen sich die Resonanzbreiten auch über die Permittivitätsdifferenz von Wellenleiter und Superstrat bzw. Substrat beeinflussen. Demnach wird für große Differenzen von $\epsilon_{1} \equiv n_{1}^{2}$ und $\varepsilon_{0} \equiv n_{0}^{2}$ bzw. $\varepsilon_{2} \equiv n_{2}^{2}$ eine Zunahme von $\Delta \lambda_{\text {FWHM }}$ erwartet MAGNUSSON und WANG 1992; WANG und Magnusson 1993). Darüber hinaus zeigt die Arbeit von ThURMAN und MORRIS (2003), dass auch bei fixen Parametern Winkel und Wellenlänge durch Variation der Wellenleiterdicke $h$ die spektrale Resonanzbreite mit ansteigendem $h$ kleiner wird.

Durch einfaches Umstellen von 2.53 bzw. 2.54 lässt sich Wellenleiterdicke $h$ der DGWS in Abhängigkeit der Polarisation darstellen. Wie man bereits an den Gleichungen (2.26) und (2.27) erkennt, ist nur der Phasenversatz durch die GoOs-HÄNCHEN-Verschiebung polarisationsabhängig, der Phasensprung durch den Gangunterschied nicht. Demnach kann $h_{\overline{\text { DGWS }}}$ in der Kurzform verallgemeinert dargestellt werden (SELLE 2003):

$$
h_{\overline{\mathrm{DGWS}}}=\frac{\lambda_{0}}{4 \pi} \cdot \frac{M \cdot 2 \pi-\bar{\Phi}_{10}-\bar{\Phi}_{12}}{\sqrt{n_{1}^{2}-\left(n_{0} \cdot \sin \theta_{0}+m \cdot \lambda_{0} / \Lambda\right)^{2}}} .
$$

Mit der in Abschnitt 4.2.3.2 charakterisierten temperaturabhängigen DGWS Probe kann die unter Temperaturänderung $\Delta \mathrm{T}$ bzw. dT hervorgerufene Resonanzverschiebung analytisch betrachtet werden. Demnach lässt sich die Temperaturabhängigkeit der Anregungswellenlänge $\lambda_{0}$ in differentieller Form wie folgt darstellen (LEITEL u. a. 2006):

$$
\frac{\mathrm{d} \lambda}{\mathrm{dT}}=\frac{\mathrm{d} \lambda}{\mathrm{d} n_{1}} \cdot \frac{\mathrm{d} n_{1}}{\mathrm{dT}}
$$

Mithilfe der Wellenleiterdicke $h_{\overline{\text { DGWS}}}$, welche nach 2.69 u. a. von $\lambda_{0}$ und $n_{1}$ abhängt, kann das (unvollständige) totale Differential von $h_{\overline{\text { DGWS }}}$ gebildet werden: 


$$
\mathrm{d} h_{\overline{\mathrm{DGWS}}} \approx \frac{\partial h_{\overline{\mathrm{DGWS}}}}{\partial \lambda} \cdot \mathrm{d} \lambda+\frac{\partial h_{\overline{\mathrm{DGWS}}}}{\partial n_{1}} \cdot \mathrm{d} n_{1}=0
$$

Durch Umstellen der Näherung 2.71 erhält man die äquivalente Gleichung:

$$
\frac{\mathrm{d} \lambda}{\mathrm{d} n_{1}}=-\left(\frac{\partial h_{\overline{\mathrm{DGWS}}}}{\partial n}\right) \cdot\left(\frac{\partial h_{\overline{\mathrm{DGWS}}}}{\partial \lambda}\right)^{-1} .
$$

Aufgrund der Komplexität der über die EMT angenäherten Formeln 2.51 und 2.52 wird der Ausdruck für h $h_{\text {DGWS }}$ nach 2.69 durch die Festlegung von verschiedenen Parametern vereinfacht: Zum einen wird der Einfallswinkel $\theta_{0}=0$ gesetzt (Normalinzidenz), zum anderen werden die Größen $M=0$ (Monomode) sowie $m= \pm 1$ gesetzt. In Abhängigkeit von der Polarisation lässt sich das Differential $\mathrm{d} \lambda / \mathrm{d} n_{1}$ zunächst für den planaren Wellenleiter ohne Gitterstruktur, d. h. für $\lim _{d \rightarrow 0} h_{\overline{\mathrm{DGWS}}}=h_{\mathrm{WL}}$, darstellen $($ STENZEL 2004; LEITEL u. a. 2006):

$$
\begin{aligned}
& \lim _{d \rightarrow 0}\left[\frac{\mathrm{d} \lambda}{\mathrm{d} n_{1}}\right]_{\mathrm{TE}}=\frac{n_{1} \cdot \Lambda^{2}}{\lambda_{0}} \cdot\left\{\left(M \cdot \pi-\frac{\Phi \frac{\mathrm{TE}}{10}}{2}-\frac{\Phi \frac{\mathrm{TE}}{12}}{2}\right)\right. \\
& \left.-\left[\frac{\tan \left(\Phi \frac{\mathrm{TE}}{10} / 2\right)}{1+\tan ^{2}\left(\Phi \frac{\mathrm{TE}}{12} / 2\right)}+\frac{\tan \left(\Phi \frac{\mathrm{TE}}{10} / 2\right)}{1+\tan ^{2}\left(\Phi \frac{\mathrm{TE}}{12} / 2\right)}\right]\right\} \\
& \cdot\left\{\left(\frac{n_{1}^{2} \cdot \Lambda^{2}}{\lambda_{0}^{2}}\right) \cdot\left(M \cdot \pi-\frac{\Phi \frac{T E}{10}}{2}-\frac{\Phi \frac{T E}{12}}{2}\right)\right. \\
& \left.-\left[\frac{1}{\tan \left(\Phi \frac{T E}{10} / 2\right)}+\frac{1}{\tan \left(\Phi \frac{T E}{12} / 2\right)}\right]\right\}^{-1} \text {, }
\end{aligned}
$$




$$
\begin{aligned}
\lim _{d \rightarrow 0}\left[\frac{\mathrm{d} \lambda}{\mathrm{d} n_{1}}\right]_{\frac{\mathrm{TM}}{}} & =\frac{n_{1} \cdot \Lambda^{2}}{\lambda_{0}} \cdot\left\{\left(M \cdot \pi-\frac{\Phi \frac{\mathrm{TM}}{10}}{2}-\frac{\Phi \frac{\mathrm{TM}}{12}}{2}\right)\right. \\
& \left.+\left(1-\frac{2 \cdot \lambda_{0}^{2}}{n_{1}^{2} \cdot \Lambda^{2}}\right) \cdot\left[\frac{\tan \left(\Phi \frac{\mathrm{TM}}{10} / 2\right)}{1+\tan ^{2}\left(\Phi \frac{T \mathrm{TM}}{10} / 2\right)}+\frac{\tan \left(\Phi \frac{\mathrm{TM}}{10} / 2\right)}{1+\tan ^{2}\left(\Phi \frac{\mathrm{TM}}{12} / 2\right)}\right]\right\} \\
& \cdot\left\{\left(\frac{n_{1}^{2} \cdot \Lambda^{2}}{\lambda_{0}^{2}}\right) \cdot\left(M \cdot \pi-\frac{\Phi \frac{\mathrm{TM}}{10}}{2}-\frac{\Phi \frac{\mathrm{TM}}{12}}{2}\right)\right. \\
& -\left[\frac{n_{1}^{4}+n_{0}^{4} \cdot \tan ^{2}\left(\Phi \frac{\mathrm{TM}}{10} / 2\right)}{n_{0}^{4} \tan \left(\Phi \frac{\mathrm{TM}}{10} / 2\right) \cdot\left(1+\tan ^{2}\left(\Phi \frac{\mathrm{TM}}{10} / 2\right)\right)}\right. \\
& \left.\left.+\frac{n_{1}^{4}+n_{2}^{4} \cdot \tan ^{2}\left(\Phi \frac{\mathrm{TM}}{12} / 2\right)}{n_{2}^{4} \cdot \tan \left(\Phi \frac{\mathrm{TM}}{12} / 2\right) \cdot\left(1+\tan ^{2}\left(\Phi \frac{\mathrm{TM}}{12} / 2\right)\right)}\right]\right\}^{-1} .
\end{aligned}
$$

Wie eine umfassende Betrachtung von $\mathrm{d} \lambda / \mathrm{d} n_{1}$ nach EMT d. h. $d \neq 0$, gezeigt hat, ist im Vergleich zu 2.73) und 2.74) durch Einsetzen von $d=18 \mathrm{~nm}$ (vgl. Probe DGWS/Hf331/18 in Abschnitt 4.2.3.2 sowie weiterer realistischer Variablengrößen lediglich eine Abweichung von $<1 \%$ zu erwarten. Auf die vollständige Darstellung der mittels EMT erweiterten und vergleichsweise komplexen Formel (2.72) wird daher verzichtet.

\subsubsection{Intensitätsverhalten in Transmission und Reflexion}

Wie bereits in Kapitel 1 erwähnt wird, kann das Resonanzphänomen der DWG als Interferenzeffekt verstanden werden (AvRUTSKY u. a. 1989). Mithilfe der Vielstrahlinterferenz, bei der die Überlagerung (Superposition) aller gebeugten Teilstrahlen betrachtet wird, ist es möglich, das Resonanzverhalten einer einfallenden Welle in Transmission und Reflexion zu beschreiben.

Unter der Annahme eines idealen Gitters unendlicher Ausdehnung lässt sich das E-Feld nach Addition aller interferierenden Teilstrahlen als geometrische Reihe darstellen (ShaRON u. a. 1997b; RosenblatT u. a. 1997). Danach lautet die auf die Amplitude $E_{0}$ normierte Funktion für die E-Feldverteilung in Transmission $E_{T}$ : 


$$
\frac{E_{T}}{E_{0}}=1-\frac{\eta \cdot \mathrm{e}^{\mathrm{i} \Phi_{\overline{\mathrm{DWG}}}}}{1-(1-\eta) \cdot \mathrm{e}^{\mathrm{i} \Phi_{\overline{\mathrm{DWG}}}}}
$$

bzw. in Reflexion $E_{R}($ JACOB u. a. 2000, 2001b):

$$
\frac{E_{R}}{E_{0}}=\frac{\eta \cdot \mathrm{e}^{\mathrm{i} \Phi \overline{\mathrm{DWG}}}}{1-(1-\eta) \cdot \mathrm{e}^{\mathrm{i} \cdot \Phi_{\overline{\mathrm{DWG}}}}}
$$

Die Phase $\Phi_{\overline{\mathrm{DWG}}}$ bezeichnet die polarisationsabhängige Gesamtphase der DWG und kann in Abhängigkeit der vorliegenden Struktur GWS bzw. DGWS unter Berücksichtigung der EMT unterschiedlich ausfallen [vgl. 2.47) und (2.47) bzw. (2.53) und 2.54)]. Der Diffraktionskoeffizient $\eta$ ist hingegen unabhängig von der Polarisation und äquivalent zur Beugungseffizienz des zur Modenanregung gebeugten Teilstrahls (i. d. R. $m= \pm 1$ ) bestimmt. In Abhängigkeit von der verwendeten Struktur wird $\eta$ unterschiedlich berechnet (s.u.).

Die Verteilung der Intensität in Transmission lässt sich normiert auf die Intensität der einfallenden Welle wie folgt beschreiben (SHARON u. a. 1995):

$$
\frac{I_{T}}{I_{0}}=\left(\frac{E_{T}}{E_{0}}\right) \cdot\left(\frac{E_{T}}{E_{0}}\right)^{*}=\frac{4 \cdot(1-\eta) \cdot \sin ^{2}\left(\Phi_{\overline{\mathrm{DWG}}} / 2\right)}{\eta^{2}+4 \cdot(1-\eta) \cdot \sin ^{2}\left(\Phi_{\overline{\mathrm{DWG}}} / 2\right)}
$$

Analog lässt sich die Gleichung für die Intensität in Reflexion $I_{R}$ aufstellen, wobei der Verlauf der Funktion mit $I_{R}=1-I_{T}$ ein sog. LoRENTz-Profil (NORTON u. a. 1997) zeigt:

$$
\frac{I_{R}}{I_{0}}=\left(\frac{E_{R}}{E_{0}}\right) \cdot\left(\frac{E_{R}}{E_{0}}\right)^{*}=\frac{\eta^{2}}{\eta^{2}+4 \cdot(1-\eta) \cdot \sin ^{2}\left(\Phi_{\overline{\mathrm{DWG}} / 2} / 2\right)}
$$

Vergleicht man 2.77) und 2.78 mit den sog. AIRY-Funktionen des FABRYPÉRot-Interferometers - kurz FPI - (FABRY und PÉROT 1899), so zeigt sich, 
dass die Intensitäten $I_{T}$ und $I_{R}$ der DWG gerade invertiert sind zu denen des FPI (KAPPEL u. a. 2004b):

$$
\begin{aligned}
& \left(\frac{I_{T}}{I_{0}}\right)_{\text {DWG }} \sim\left(\frac{I_{R}}{I_{0}}\right)_{\text {EPT }}, \\
& \left(\frac{I_{R}}{I_{0}}\right)_{\text {DWG }} \sim\left(\frac{I_{T}}{I_{0}}\right)_{\text {EPI }} .
\end{aligned}
$$

Die bei der Darstellung der AIRY-Funktionen des FPI mit zunehmendem Spiegelabstand auftretenden Periodizität von $2 \pi$ (LAUTERBORN u. a. 1993, BORN und WOLF 1999) kann bei der DWG vollkommen analog durch die Zunahme der Wellenleiterdicke $h$ und der Anregung höherer Schwingungsmoden betrachtet werden (SELLE 2003). Die beim FPI als Finessekoeffizient bekannte Größe $F$, welche durch die Reflektivität $R$ der Spiegel bestimmt ist:

$$
F=\frac{4 R}{(1-R)^{2}}
$$

kann bei Betrachtung der Formeln 2.77 und 2.78 über die Beziehung $R \stackrel{\text { def }}{=} 1-\eta$ gleichgesetzt werden.

\subsection{Numerische Berechnungen sowie ergänzende Beispielsimulationen mithilfe rigoroser Methoden}

Auf der Grundlage analytischer Formeln der Abschnitte zuvor werden in diesem Unterkapitel einige ausgewählte numerische Beispielrechnungen gezeigt. Sowohl zum direkten Vergleich diese Berechnungen als auch in Ergänzung zur vorangegangenen Theorie werden zusätzlich zwei rigorosen Methoden verwendet, durch die es mittels vollständiger Lösung der MAxwELLschen Gleichungen möglich wird, die Feld- bzw. Intensitätsverteilung einer DWG unter Resonanzbedingungen darzustellen. Einer dieser Methoden ist die rigorose Beugungstheorie (engl. Rigorous 
coupled-wave analysis, kurz RCWA, welche die Strukturgeometrie einer DWG. einschließlich der Gitterstruktur, in einzelne Schichten bzw. Quader konstanter Brechzahlen unterteilt und diese dann durch die Entwicklung von FOURIER-Reihen in Form eines Eigenwertproblems (S-Matrix-Methode) semi-analytisch löst (MOHARAM u. a. 1995a, b). Bedingt durch die spezifische Zerlegung (Diskretisierung) werden Gitterstrukturen mit einem Sinusprofil mithilfe der RCWA nur approximativ berechnet. Zudem unterstützt das zur RCWA-Simulation verwendete kommerzielle Computerprogramm (FLUCKIGER 2004) ausschließlich ebene Wellen unter der Annahme idealer, lateral unendlich ausgedehnter Strukturen.

Das zweite Programm, welches zur Darstellung der Feldverteilung bei resonanter Anregung der DWG-Strukturen verwendet wird, ist ebenfalls kommerziell erhältlich (COMSOL 2008) und löst die MAXWELL-Gleichungen approximativ durch die sog. Finite-Elemente-Methode - kurz FEM (JIN 2002). Während bei den Berechnungen mittels RCWA die lateralen Ausdehnungen der Struktur als unendlich angenommen werden, ist das Rechengebiet bei der FEM zur Lösung partieller Differentialgleichungen durch verschiedene Randbedingungen begrenzt (s. u.).

Die in diesem Abschnitt gezeigten Simulationen werden anhand einer DGWS demonstriert, deren Strukturparameter durch die Gitterperiode $\Lambda=360 \mathrm{~nm}$, die Wellenleiterdicke $h=110 \mathrm{~nm}$ und die Gittertiefe $d=40 \mathrm{~nm}$ gegeben sind. Der Füllfaktor $a / \Lambda$ beträgt 0,5 und die Brechzahlen der DGWS sind auf $n_{0}=1$ (Superstrat), $n_{1}=2,085$ (Wellenleiter) und $n_{2}=1,52$ (Substrat) festgelegt.

Abb. 2.7 zeigt die auf der Grundlage von 2.53) und 2.54 berechneten sog. Resonanzkennlinien, welche die Anregung der DGWS für TE bzw. TM.polarisiertes Licht durch die Verknüpfung von Resonanzwinkel bzw. -wellenlänge darstellen. Die mittleren Brechzahlen $\bar{n}_{10 \text { TE }}$ und $\bar{n}_{10 \text { TM }}$ sind je nach Polarisation mithilfe der Formel 2.49 bzw. 2.50 bestimmt worden. Die Anregung der gezeigten Schwingungsmoden $\mathrm{TE}_{1}, \mathrm{TE}_{1}, \mathrm{TM}_{3}$ und $\mathrm{TM}_{1}$ erfolgt in dieser Darstellung ausschließlich über die \pm 1 . Beugungsordnung. Anders als in der von Wang und MAGNUSSON (1993) verwendeten Darstellung ist in Abb. 2.7 das Vorzeichen der Beugungsordnung, d. h. die Propagationsrichtung der geführten Welle, im Winkelspektrum von $-90^{\circ}$ bis $+90^{\circ}$ auf der Ordinate enthalten (vgl. Abb. 4.1a). Zusätzlich zu den analytischen Berechnungen sind auch selektierte, mittels $\overline{R C W A}$ berechnete Kennlinienpunkte dargestellt, welche für den gezeigten Wellenlängenbereich von 200-1000 nm eine 


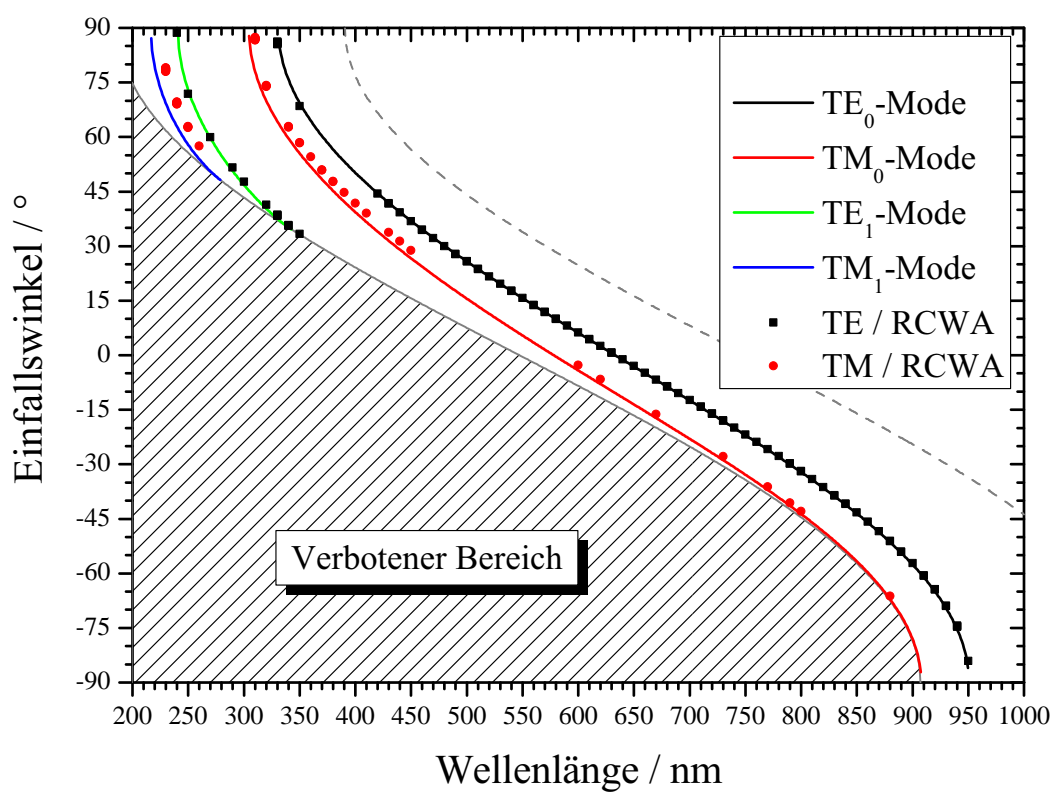

Abb. 2.7: Darstellung vom Zusammenhang der Resonanzwellenlänge und -winkel (Resonanzkennlinie) einer DGWS für TE bzw. TM-polarisiertes Licht. Ausschließlich im Propagationsbereich der Schwingungsmoden (Resonanzkorridor) ist eine Anregung der Moden durch die \pm 1 . Beugungsordnung möglich.

Transmittivität von $\leq 1 \%$ aufweisen. In Anbetracht der guten Übereinstimmung von Modell und RCWA insbesondere für TE-polarisiertes Licht scheint die durch Formel 2.49) getroffene Annahme zur effektiven Gitterbrechzahl zur Beschreibung der Resonanzverschiebung bei einer Änderung der Wellenlänge bzw. des Winkel für eine Vorhersage hinreichend genau zu sein. Die in 2.50 gezeigte Näherung für die TM-Anregung scheint indes besonders für kleinere Wellenlängen zu großen Abweichungen gegenüber der RCWA-Rechnung zu führen.

Die in Abb. 2.7 als »Verbotener Bereich« markierte Fläche kennzeichnet das Gebiet, in dem für die 1. Beugungsordnung keine Totalreflexion am Medienübergang von $n_{1}$ (Wellenleiter) nach $n_{2}$ (Substrat) mehr stattfinden kann. Die Grenze zwischen dem verbotenen Bereich und dem sog. Resonanzkorridor, welcher den Propagationsbereich der Moden vorgibt, ist durch die untere Grenze in 2.46 gegeben; die obere Grenze ist in Abb. 2.7 als gestrichelte Linie dargestellt. Fallen die Kennlinien der Schwingungsmoden $\mathrm{TE}_{1}$ und $\mathrm{TM}_{1}$ also mit den Grenzen des Korridors zusammen, werden die \pm 1 . Beugungsordnungen nicht mehr total-reflektiert, son- 
dern treten in das darunter liegende Substratmedium ein, was mit einem Abbruch der Wellenleitung verbunden ist. Die in Abb. 2.7 gezeigte spektrale Breite des Resonanzkorridors bleibt mit den gegebenen Strukturparametern für einen beliebigen Einfallswinkel konstant bei 203,4 nm. Unter Berücksichtigung wellenlängenabhängiger Brechungsindizes (Dispersion) können sich jedoch die Form sowie die Breite des Korridors mit dem Winkel ändern (SELLE 2003).

Liegt die Anregungswellenlänge $\lambda_{0}$ nicht mehr innerhalb der Grenzen nach Formel (2.46), so kann es für $\lambda_{0}<\left(\max \left\{n_{0}, n_{2}\right\} \mp n_{0} \cdot \sin \theta_{0}\right) \cdot \Lambda$ zur Resonanzanregung durch höhere Beugungsordnungen $|m|>1$ kommen. So zeigt z. B. Abb. 4.5 die berechneten, auf die Strukturparameter der Probe DGWS/Hf74/40 angepassten Kennlinien der 2. Beugungsordnung. Zusammen mit den gezeigten Grenzen helfen diese zu verstehen, warum die bei der Wellenlänge $355 \mathrm{~nm}$ charakterisierte Probe bei der Anregung mit TM-polarisiertem Licht eine vergleichsweise geringe Reflektivität zeigt (vgl. Abb. 4.4b).

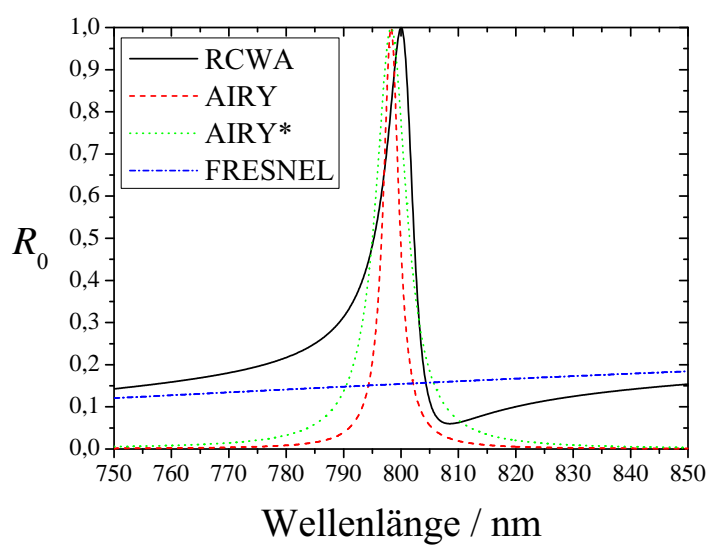

Abb. 2.8: Bei einem Resonanzwinkel von $-31,95^{\circ}$ berechnete Reflektivität 0 . Ordnung $\left(R_{0}\right)$ einer DGWS mit rigoroser Beugungstheorie (RCWA) und analytischem Modell der Vielstrahlinterferenz (AIRY).
Zusätzlich zum gezeigten Verhalten von Resonanzwellenlänge und - winkel lassen sich auch die Intensitäten der DGWS nach gegebenen Strukturparametern (s. o.) berechnen. Abb. 2.8 zeigt die berechnete Reflektivität nullter Ordnung $R_{0}$ mit rigoroser Beugungstheorie RCWA und analytischem Modell der Vielstrahlinterferenz (AIRY) für TE-polarisiertes Anregungslicht unter dem Einfallswinkel $-31,95^{\circ}$. Dabei liegt die analytisch berechnete Resonanzwellenlänge für diesen Winkel um 1,7 nm zu kleineren Wellenlängen verschoben. Für TM-polarisiertes Licht (Daten nicht gezeigt) beträgt die Wellenlängendiskrepanz, wie bereits in Abb. 2.7 angedeutet, für einen Resonanzwinkel von $-42,9^{\circ}$ sogar $3,36 \mathrm{~nm}$. Die entsprechende Winkeldis- 


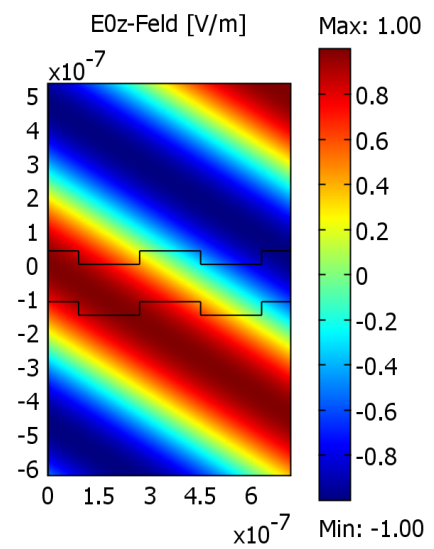

(a) E-Feld der einfallenden Welle.

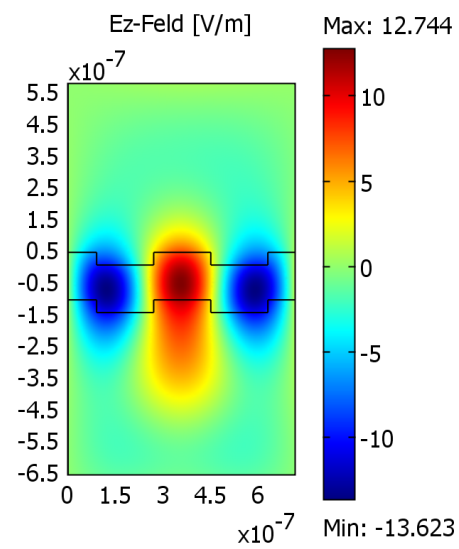

(b) E-Feld der gestreuten Welle.

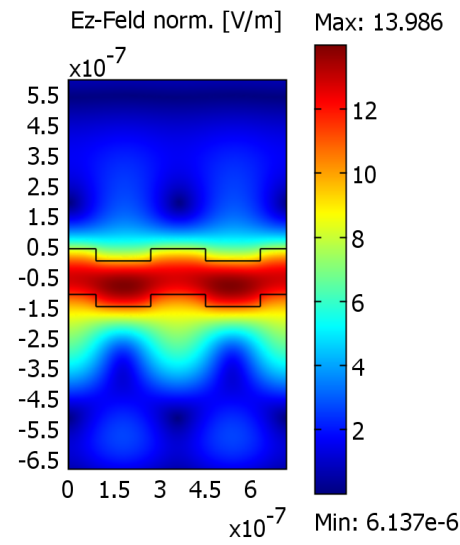

(c) Normiertes E-Feld der gestreuten Welle.

Abb. 2.9: FEM-Simulationen zur E-Feldverteilung bei resonanter DGWS-Anregung durch eine TE polarisierte ebene Welle der Wellenlänge $800 \mathrm{~nm}$ unter $-31,82^{\circ}$ Einfall.

krepanz lässt sich für TM-Anregung auf $0,78^{\circ}$ bzw. für TE Anregung auf $0,2^{\circ}$ beziffern.

Die in Abb. 2.8 dargestellten AIRY-Funktionen zeigen nach Formel (2.78) ein symmetrisches Reflexionsprofil (LoRENTZ-Profil), wobei die grüne, breitere der beiden Funktionen (AIRY*) mithilfe des Diffraktionskoeffizienten nach Formel 2.65) berechnet worden ist. Mit einer Halbwertsbreite ( $\overline{\text { FWHM }}$ ) von $6,5 \mathrm{~nm}$ stimmt diese mit der mittels RCWA berechneten Intensitätsverteilung überein. Der rote AIRY-Graph hingegen gibt das spektrale Verhalten unter Vernachlässigung des Phasenversatzes $\delta$ wieder und ist im Vergleich zu AIRY* mit einer FWHM von 3,2 nm ungefähr halb so breit. Die bei der RCWA aufgrund des schrägen Einfalls hervorgerufene Asymmetrie wird bei der analytischen Betrachtung vernachlässigt. Darüber hinaus wird auch der FRESNEL-Anteil (blau), welcher das Reflexionsvermögen der DGWS ohne Gitterstruktur angibt, nicht berücksichtigt.

Um eine Vorstellung über die Feldverteilung innerhalb bzw. außerhalb der DGWS unter Resonanzbedingungen zu erhalten, zeigt Abb. 2.9 die FEM-Simulationen zur E-Feld-Verteilung für eine zeit-harmonische, linear polarisierte ebene Welle der Wellenlänge $800 \mathrm{~nm}$ bei TE-polarisiertem Lichteinfall. Mit einem Resonanzwinkel von $-31,82^{\circ}$ zeigt Teilabb. 2.9a das E-Feld der einfallenden Welle, während 2.9b 
und 2.9c die gestreute Welle, d. h. die E-Feldverteilung nach Abzug der einfallenden Welle, zeigen; im Unterschied zu2.9b zeigt 2.9cjedoch das auf den Betrag der Welle normierte Feld.

Die Diskretisierung des zweidimensionalen Gebietes der DGWS, welches eine Ausdehnung von $720 \mathrm{~nm}$ (2 Gitterperioden) in x-Richtung und ca. 1,2 $\mathrm{mm}$ in yRichtung besitzt, erfolgt durch Tetraeder-Elemente (nicht gezeigt), an deren Knoten die partiellen Differentialgleichungen durch LAGRANGE-Funktionen 2. Ordnung gelöst werden. Mithilfe einer periodischen Randbedingung, der sog. FLOQUET-Periodizität (ZHU und CANGELlARIS 2006) lässt sich die horizontale Ausdehnung des Rechengebietes auf zwei Perioden (theoretisch sogar bis auf eine Periode) beschränken. Darüber hinaus wird durch die Verwendung einer spezifischen absorbierenden Schicht, einer sog. Perfectly matched layer - kurz PML $-(\overline{B E R E N-}$ GER 1994), eine E-Feld-Rückstreuung an den Grenzflächen oben und unten (nicht gezeigt) vermieden. Die Winkeldiskrepanz von $-0,13^{\circ}$ zwischen RCWA und FEM ist auf Approximationen beider Methoden zurückzuführen.

Neben der Berechnung von elektromagnetischen Feldern lässt sich mithilfe von FEM-Simulationen auch die Verteilung der Energiedichte bestimmen (s. Abb. 5.8), wodurch eine Abschätzung zur Zwei-Photonen-Fluoreszenzanregung von Farbstoffmolekülen auf einer DGWS-Oberfläche möglich ist (s. Abschnitt 5.2).

Weiterführende und zum Teil - da animiert - recht anschauliche RCWASimulationen, z. B. zum Einfluss der Wellenleiterdicke auf das Resonanzverhalten unter Berücksichtigung der WooDschen Anomalien, finden sich in der Arbeit von RUMPF und Johnson (2007). Darüber hinaus zeigen HEGER (2005) sowie BunKOWSKI u. a. (2006) verschiedene RCWA-Rechnungen zum Einfluss des Füllfaktors auf die Resonanz bei TE- bzw. TM-Anregung. FEM-Simulationen zur Reflexion und Transmission geführter Moden an kurzen dielektrischen Reliefgittern auf planaren Wellenleitern werden in der Dissertation von HimmLER (2006) gezeigt. 


\section{Herstellung dielektrischer Wellenleitergitter}

Dieses Kapitel widmet sich der Herstellung dielektrischer Wellenleitergitter (DWG) und gliedert sich wie folgt: Abschnitt 3.1 befasst sich mit den unterschiedlichen Strukturformen der DWG und erörtert die Möglichkeiten einer Resonanzverschiebung nach Fertigung der Struktur. Anschließend geht Abschnitt 3.2 kurz auf die lithographischen Verfahren der Gittererzeugung und ausführlich auf die Herstellung von Gitterstrukturen mithilfe der $\mathrm{F}_{2}$-Laserablation ein. Weiterhin erläutert Abschnitt 3.3 die Beschichtungsverfahren, die zur Herstellung der wellenleitenden Schichten relevant sind. Im Anschluss beschreibt Abschnitt 3.4 die OberflächenCharakterisierung einzelner DWG-Proben. Das Kapitel schließt mit Abschnitt 3.5 , in dem alle gefertigten Proben und ihre wichtigsten Strukturdaten zusammengefasst sind.

\subsection{Strukturunterschiede dielektrischer Wellenleitergitter}

Aufgrund verschiedener Ansätze bei der Fertigung dielektrischer Wellenleitergitter sind nach Abschluss der Strukturbildung unterschiedliche Strukturformen erkennbar. Bei den im Rahmen dieser Arbeit verwendeten DWG-Proben werden zwei grundlegende Strukturformen unterschieden, deren Fertigungsansätze nachfolgend beschrieben sind.

Im ersten Ansatz wird zunächst eine Gitterstruktur auf der Oberfläche eines Trägersubstrats erzeugt, welche im Anschluss mit einem wellenleitenden Material beschichtet wird. Aufgrund des gewählten Beschichtungsprozesses (s. Abschnitt 3.3 bleibt nach der Beschichtung ein Abbild des zuvor erzeugten Gitterreliefs an der Oberfläche des Wellenleiters erhalten. Die abschließende Strukturform wird als sog. Doppel-Gitter-Wellenleiter-Struktur (DGWS bezeichnet - einer Struktur mit je einem Gitterprofil oberhalb und unterhalb des Wellenleiters (s. Abb. 3.1a). 


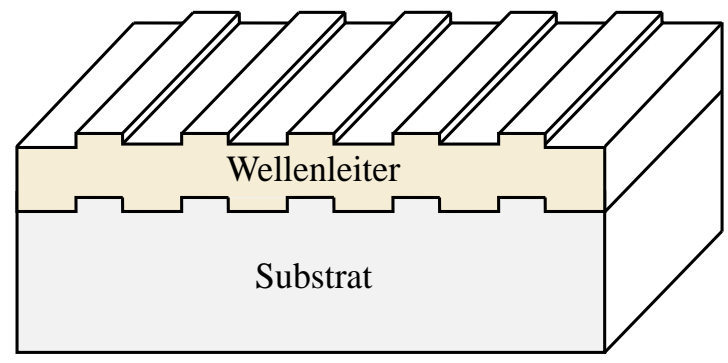

(a) DGWS mit zwei Gitterprofilen.

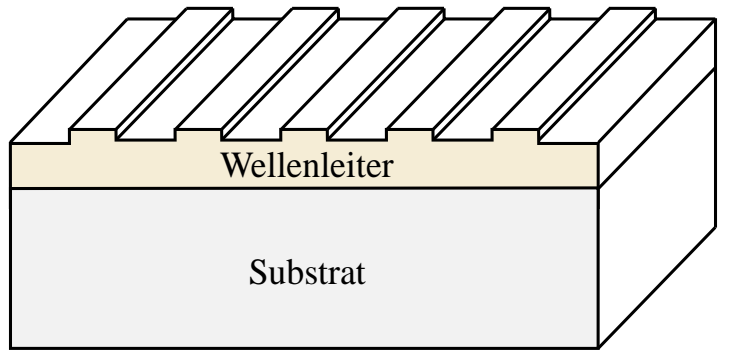

(b) GWS mit einem Gitterprofil.

Abb. 3.1: Schematische Darstellung zum Vergleich einer (a) Doppel-GitterWellenleiter-Struktur (DGWS) und (b) einer Gitter-Wellenleiter-Struktur (GWS).

Beim zweiten Ansatz wird die Reihenfolge von Gittererzeugung und Beschichtung umgekehrt: Zunächst wird das Substrat mit einem wellenleitenden Material beschichtet und im Anschluss die Gitterstruktur mit geeigneten Verfahren in die Oberfläche des Wellenleiters (s. Abschnitt 3.2.2) gefertigt. Das erzeugte Gitterprofil befindet sich dann auf der Oberseite des Wellenleiters (GWS, s. Abb. 3.1b).

Aufgrund von Toleranzen bei der Fertigung der DWG kommt es zwangsläufig und unabhängig von der strukturellen Form zu Abweichungen einzelner oder mehrerer Strukturparameter. Da sich die Schwankungen unmittelbar auf das Resonanzverhalten auswirken (s. Abschnitt 2.4.1), ist es notwendig, auftretende Fertigungstoleranzen bereits bei den Simulationen zum Strukturmodell bzw. Resonanzverhalten zu berücksichtigen. Dies setzt jedoch einen umfassenden Informationszugriff auf möglichst alle Prozessparameter der Fertigung voraus und kann bei einer Auslagerung der Fertigung an Dritte nur auf der Grundlage enger Zusammenarbeit aller Beteiligten gelingen. Da jedoch bis auf die Oberflächenstrukturierung mittels Laserablation sämtliche Fertigungsprozesse durch Industriefirmen oder von Fremdinstitutionen geleitet werden, ist ein Eingriff in die einzelnen Prozesse der im Rahmen dieser Arbeit zu fertigenden Proben nur sehr begrenzt möglich. Wie die Verknüpfung von Fertigung und Simulation aussehen kann, zeigt die Arbeit von RumpF und JoHnson (2007): Mit der Erfassung und Auswertung von Prozessdaten aus einem Beschichtungsprozess kann die aufgrund der Fertigungstoleranzen erwartete Resonanzverschiebung zunächst berechnet und anschließend durch einen gezielten Wiedereingriff in den Fertigungsprozess eine weitgehende Kompensation der Abweichung zugunsten der angestrebten Resonanz erzielt werden. 
Dennoch, unabhängig von der Präzision der Fertigung, sind sämtliche Strukturparameter der gefertigten DWG.Proben - ob DGWS oder GWS - nach Abschluss der Strukturbildung grundsätzlich festgelegt. Bei den Proben mit einem rein passiven Resonanzverhalten kann eine Verschiebung der Resonanz nur über die direkte Kopplung von Einfallswinkel und Wellenlänge erfolgen (s. Abschnitt 2.4.1); ein nachträglicher Eingriff in das Resonanzverhalten ist daher bei einer passiven Struktur nicht mehr möglich. Mit Ausnahme einer DGWS, deren wellenleitende Schicht es erlaubt, den Brechungsindex der Schicht und damit die Resonanz durch Temperaturänderung zu verschieben, besitzen alle im Rahmen dieser Arbeit verwendeten Proben eine solche passive Struktur (s. Abschnitt 3.5).

Mit der Erzeugung einer veränderbaren Strukturgröße, d. h. der Generierung eines Freiheitsgrads innerhalb der resonanten Struktur, ist es jedoch möglich, das Resonanzverhalten nach der Fertigung zu beeinflussen. In der Literatur finden sich bereits Ansätze, die mithilfe eines aktiven Strukturparameters eine Steuerung der Resonanz ermöglichen. Mit dem Einsatz von Halbleitermaterialien innerhalb resonanter Wellenleitergitter gelingt es SHARON u.a. (1996a) durch die Erzeugung eines äußeren elektrischen Feldes eine Intensitätsmodulation des oberflächenreflektierten Laserstrahls zu erzielen. Die verwendete Struktur besteht jedoch nicht wie bei der DWG aus nur einer optischen Schicht (ohne Substrat), sondern setzt sich zu einem Strukturverbund vieler Halbleiterschichten zusammen. Wie auch die Arbeit von DudOvich u.a. (2001) zeigt, hat diese vielschichtige Struktur den Vorteil, besonders spektral schmalbandige Resonanzen im Subnanometerbereich zu erzeugen, wobei außerdem eine Resonanzverschiebung von knapp einem halben Nanometer erreicht wird. Darüber hinaus kann die Resonanzwellenlänge mithilfe eines speziellen elektro-optischen Polymers in Abhängigkeit der angelegten elektrischen Spannung um bis zu $2 \mathrm{~nm}$ verschoben werden (KATCHALSKI u. a. 2005a).

Zudem wird auch die optische Nichtlinearität von Materialien ausgenutzt, um über die Resonanzverschiebung einen optischen Schaltvorgang, z. B. mithilfe nichtlinearer Polymer-Wellenleiter (BADER 2001, 2003), zu erzeugen, was jedoch nur bei hinreichend intensiver Bestrahlung der Struktur erreicht wird. Berechnungen von BOYE u. a. (1999) auf der Basis der rigorosen Beugungstheorie (RCWA und der finiten Differenzen-Methode (FDTD haben gezeigt, dass mit optischen Strahlungsdichten von unter $100 \mathrm{~kW} / \mathrm{cm}^{2}$ eine hinreichende Brechzahländerung erzeugt 
werden kann, um im Sinne eines optischen Schaltvorgangs einen Wechsel von vollständiger Transmission zu vollständiger Reflexion zu erreichen.

Im Rahmen dieser Arbeit wird eine weitere Möglichkeit vorgestellt, einen aktiven und damit veränderbaren Strukturparameter zu erzeugen: Mithilfe eines spezifischen Beschichtungsprozesses gelingt es (s. Abschnitt 3.3), eine poröse $\mathrm{HfO}_{2^{-}}$ Schicht zu erzeugen, die es ermöglicht, dass sich Wasser aus der Umgebung in die Struktur der wellenleitenden Schicht einlagert. Bei Erhöhung der Probentemperatur kann das Wasser (teilweise) verdampfen, was eine Änderung der Brechzahl zur Folge hat: Aufgrund des geringen Brechungsindexes des Wassers von ca. 1,33 in Bezug auf das Wellenleitermaterial $\mathrm{HfO}_{2}$ mit einem Index von über 2 ergibt sich für den Wellenleiter ein mittlerer Brechungsindex. Mit der Verdrängung des Wassers durch einen Temperaturanstieg werden die Zwischenräume in zunehmendem Maße durch Luft aus der Umgebung besetzt. Da jedoch die Brechzahl der Luft kleiner als die des Wassers ist, ändert sich auch der mittlere Brechungsindex der Schicht. Bei der Charakterisierung einer temperaturabhängigen DWG in Abschnitt 4.2.3.2 ist durch die Änderung der Brechzahl mit der Temperatur eine spektrale Verschiebung der Resonanz messbar und unterstützt die vorausgegangenen theoretischen Überlegungen in Abschnitt 2.4.2.

\subsection{Erzeugung der Gitterstrukturen}

Für die Erzeugung von Gitterstrukturen auf Materialoberflächen existieren vielfältige und sehr unterschiedliche technische Verfahren, die jedoch besonders in der industriellen Fertigung durch lithographische Methoden geprägt sind. Neben den lithographischen Verfahren spielt die Laser- oder Photoablation vor allem bei der Erzeugung einer GWS eine wichtige Rolle. In Abschnitt 3.2.2 wird dieses Verfahren, welches die Oberfläche durch einen Materialabtrag (Ablation) mittels Laserstrahlung moduliert, am Beispiel des Wellenleitermaterials Niobpentoxid $\left(\mathrm{Nb}_{2} \mathrm{O}_{5}\right)$ beschrieben. Besonderes Merkmal ablatierter Strukturen ist das nahezu sinusförmige Gitterprofil, welches i.d.R. entsteht, wenn die Ablation der Gitterstruktur nahe an der Auflösungsgrenze des verwendeten Systems durchgeführt wird (s. Abschnitt 3.4. 


\subsubsection{Lithographische Verfahren}

Zu den gebräuchlichen lithographischen Verfahren gehören die Elektronenstrahlbzw. Ionenstrahllithographie sowie die Photolithographie. Letztere hat sich besonders in der Halbleiterindustrie etabliert und dient dort vornehmlich der Übertragung von integrierten Schaltungen auf dünne Silizium-Wafer.

Die Photolithographie, die auch zur Strukturierung der Substratoberfläche von DGWS-Proben verwendet wird, stellt im Rahmen dieser Arbeit ein wichtiges optisches Strukturierungsverfahren dar. Durch die Belichtung einer Fotomaske, welche die Strukturinformation des Gitters enthält, wird die Gitterinformation in Form eines latenten Abbildes innerhalb des sog. Fotolacks, welcher zuvor mittels Rotationsbeschichtung (engl. spin-coating) auf die Substratoberfläche gebracht wurde, übertragen. Nach der anschließenden Entwicklung des Bildes wird der belichtete Teil des Fotolacks von der Substratoberfläche wieder entfernt. Bei einem anschließenden Ätzprozess (subtraktive Methode) wird nun die Gitterstruktur an den lackfreien Stellen in das Substrat übertragen. Über die Dauer des Ätzens kann darüber hinaus die Gittertiefe des erzeugten Profils gesteuert werden. In einem letzten Schritt wird schließlich der restliche (unbelichtete) Fotolack von der Oberfläche entfernt. Charakteristisch für die mittels Photolithographie erzeugten Gitterstrukturen ist ein nahezu ideales Rechteckprofil (Laminargitter).

Alle im Rahmen dieser Arbeit verwendeten DGWSłProben sind ausschließlich mit dem photolithographischen Verfahren strukturiert worden und wurden als vorstrukturierte Substratproben bei der Firma OC OERLIKON BALzERs ehemals UnAXIS BAlzers AG käuflich erworben (WIKI und MAISENHÖLDER 2004). Darüber hinaus hat sich die Photolithographie zur Strukturierung von Substraten, welche innerhalb der Sensortechnik mit der englischen Bezeichnung wavelengthinterrogated optical sensors WIOS verwendet werden, bereits bewährt WIKI und KunZ 2000) und erlaubt zudem die Fertigung von Mikrostrukturen, deren Strukturbreiten deutlich unter $200 \mathrm{~nm}$ liegen können (COTTIER u. a. 2002).

Eine weitere jedoch nur indirekt genutzte Strukturierungsmethode ist die Ionenstrahl- bzw. Elektronenstrahl-Lithographie. Bei diesem Verfahren wird ebenfalls ein Fotolack auf ein Trägersubstrat gebracht, der jedoch diesmal durch einen 
fokussierten Elektronenstrahl (Ionenstrahl) von hochenergetischen Elektronen (Ionen) belichtet wird. Diese direkt-schreibende (serielle) Methode wird vorwiegend bei der Herstellung von Fotomasken für die Photolithographie oder Laserablation verwendet, kommt jedoch selbst ohne Masken-Abbildung aus (SUZUKI 2007).

\subsection{2 $\quad \mathrm{F}_{2}$-Laserablation}

Eine Methode zur Strukturierung von Oberflächen mit nur einem Prozessschritt stellt die Laser- oder Photoablation dar, bei der mithilfe intensiver Laserstrahlung ein Materialabtrag an der Probenoberfläche erzeugt wird (WEHNER und IHLEMANN 2005). Dazu wird eine Gittermaske von einem geeigneten Laser beleuchtet und über eine Abbildungsoptik auf die Oberfläche der Probe projiziert. Unter anderem wird hierfür die Strahlung verschiedener UV Wellenlängen gepulster Gaslaser vom Typ Excimerlaser verwendet. Weit verbreitete Excimer-Wellenlängen sind z. B. $248 \mathrm{~nm}$ und $193 \mathrm{~nm}$, die bei der Anregung der Edelgas-Halogenide Kryptonfluorid (KrF) bzw. Argonfluorid (ArF) emittiert werden (FIEBIG 2001).

Durch die Verwendung vergleichsweise kurzer Excimer-Wellenlängen mit Pulslängen im ein- bis zweistelligen Nanosekundenbereich ist es möglich, den bei der Ablation auftretenden Wärmetransport innerhalb des Materials gering zu halten. Wie die Arbeit von PISSADAKIS u. a. (2004) zeigt, ist die Strukturierung beispielsweise von Tantalpentoxid $\left(\mathrm{Ta}_{2} \mathrm{O}_{5}\right)$ mit einer Wellenlänge von $248 \mathrm{~nm}$ zwar grundsätzlich möglich, jedoch aufgrund der Nanosekundenpulse zur Herstellung von Gittern mit Modulationstiefen von mehr als $13 \mathrm{~nm}$ nicht mehr geeignet.

Für eine präzise Strukturierung optisch-transparenter Materialien ist es außerdem notwendig, dass das zu bearbeitende Material eine hinreichende Absorption bei der verwendeten Wellenlänge besitzt. Am Beispiel von Quarzglas, welches die optische Eigenschaft hat, über einen weiten Spektralbereich transparent und damit absorptionsarm zu sein, liegt die Absorptionskante im tiefen UV-Spektralbereich, was gerade bei einer Bestrahlung mit langwelligem Licht eine entsprechend hohe Energiedichte erfordert. Die VUV-Strahlung des $\mathrm{F}_{2}$-Lasers mit einer Wellenlänge um $157 \mathrm{~nm}$ und einer Photonenenergie von rund 7,9 eV ist deshalb für die Laserablation vieler UV-transparenter Materialien besonders gut geeignet IHLEMANN u. a. 2003). 
Aufgrund der spezifischen optischen Eigenschaften absorbieren viele Metalloxide einfallende VUV-Strahlung nahezu vollständig, was speziell bei der $\mathrm{F}_{2^{-}}$ Laserstrahlung zu einer besonders kurzen optischen Eindringtiefe führt. Aufgrund der hohen Energieaufnahme durch das Material sinkt gleichzeitig die minimale Ablationsenergie (Ablationsschwelle), ab der ein Materialabtrag möglich ist, und eine besonders energieeffiziente Strukturierung von Metalloxiden wird möglich.

Im Hinblick auf die Herstellung von DWG-Proben mit der Strukturform einer GWS (s. Abschnitt 3.1) wird im Rahmen dieser Arbeit die Strukturierung einer wellenleitenden Schicht aus $\mathrm{Nb}_{2} \mathrm{O}_{5}$ erprobt. Für die Bearbeitung der $\mathrm{Nb}_{2} \mathrm{O}_{5}$-Oberfläche wird ein kommerzieller $\mathrm{F}_{2}$-Laser vom Typ LPF 220i (COHERENT ehemals LAMBDA Physik) zusammen mit einem Strahloptiksystem der Microlas LASERSYSTEM GMBH verwendet. Letzteres System besteht aus einer ca. 2,5 m langen AluminiumKammer, in der eine speziell an die Wellenlänge $157 \mathrm{~nm}$ angepasste Linsenanordnung zur optischen Strahlführung integriert ist. Mithilfe hochpräziser Probenpositionierungstische am Ausgang der Kammer stellt das gesamte System eine Bearbeitungsanlage dar, welche sich bereits bei der Erzeugung von Mikrokanälen, der Bearbeitung optischer Fasern oder der Herstellung von Zylinderlinsen bewährt hat (LI u. a. 2002).

Bei einer maximalen Repetitionsrate von $200 \mathrm{~Hz}$ liefert der Laser Energien von bis zu 25 mJ pro Puls, wobei die typische Pulsdauer etwa 15 ns beträgt. Messungen zur räumlichen Ausdehnung des Laserstrahls haben direkt am Laserausgang eine horizontale Länge von ca. $22 \mathrm{~mm}$ und eine vertikale Ausdehnung von ungefähr $5 \mathrm{~mm}$ ergeben. Die Divergenz des emittierten Laserstrahls beträgt in der Horizontalen ca. $3 \mathrm{mrad}$ und in der Vertikalen ungefähr $1 \mathrm{mrad}$. Ein NESLAB-Kühlsystem der Firma Thermo Fisher ScIEntific hält die Temperatur des Lasers annähernd konstant bei $35^{\circ} \mathrm{C}$ und sorgt im laufenden Betrieb für eine stabile Laserleistung.

Abb. 3.2 zeigt den optischen Strahlengang innerhalb des vollständig mit Stickstoff gefluteten Kammersystems zusammen mit dem Probenhalter, der sich außerhalb auf einem 3-Achsen-Positionierungssystem (xyz-Verschiebetische) befindet. Der $\mathrm{F}_{2}$-Laser (nicht gezeigt) ist direkt mit dem Kammersystem verbunden (in Abb. 3.2 oben), an dessen Eingang der emittierte Laserstrahl auf einen Abschwächer trifft, welcher die Energie des einfallenden Strahls regelt. Über eine Strahl- 


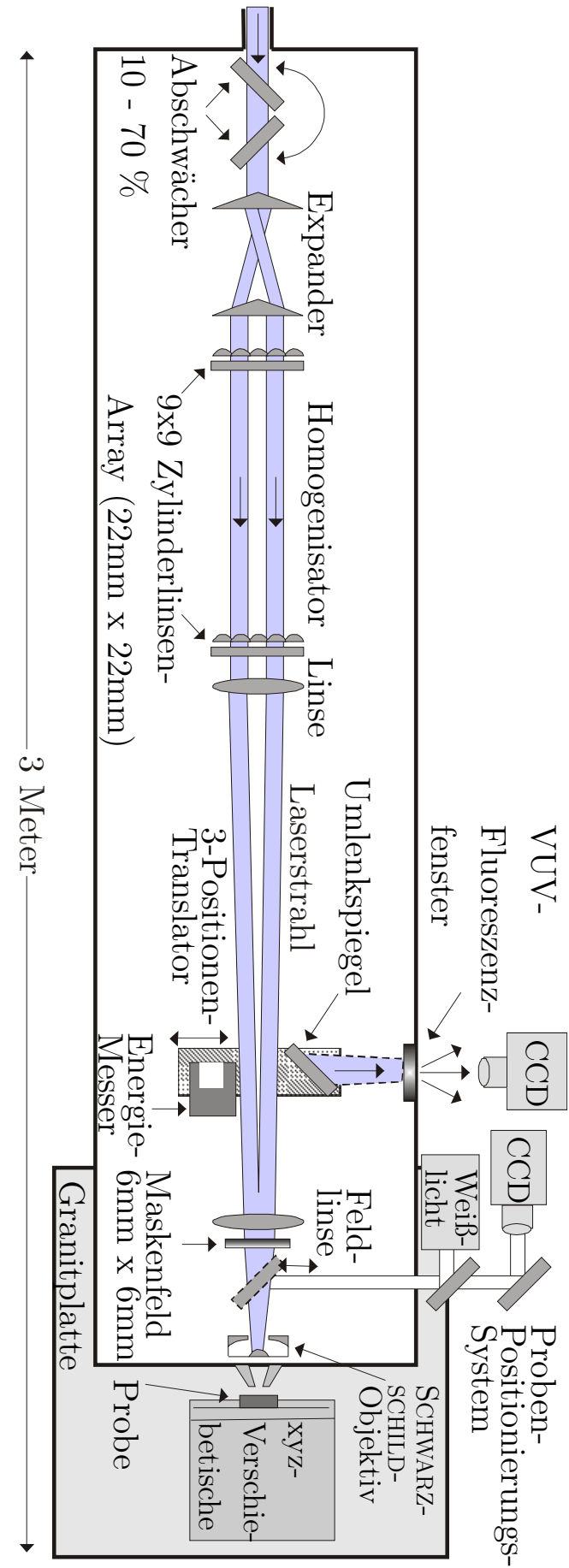

Abb. 3.2: Aufbau der Bearbeitungsanlage zur $\mathrm{F}_{2}$-Laserablation. aufweitungsoptik gelangt der Laserstrahl zu einem Strahlhomogenisator, der aus zwei Linsenmatrizen zu je $9 \times 9$ Zylinderlinsen besteht. Dieser formt das räumliche Profil des Laserstrahls zu einem Rechteck und sorgt über den gesamten Querschnitt für eine gleichmäßige Intensitätsverteilung. Ein verschiebbarer Umlenkspiegel projiziert bei Bedarf den homogenisierten Strahl auf ein sog. Fluoreszenz-Fenster, welches die ankommende Laserstrahlung über eine fluoreszierende Schicht in sichtbares Licht wandelt und für das Fluoreszenzlicht selbst transparent ist. Eine Kameraoptik bildet das erzeugte Fluoreszenzlicht auf einer CCD ab und ermöglicht so eine Strahlprofilanalyse der VUV Strahlung. Der optische Weg vom Umlenkspiegel zum Fenster ist dabei gerade so lang, dass er der Distanz zwischen Spiegel und Maskenebene entspricht. Die Uniformität des homogenisierten Strahlprofils in der Maskenebene konnte so auf $\pm 5 \%$ bestimmt werden. Die Maske wird über ein sog. SCHWARZSCHILD-Objektiv, das am Ende des Kammersystems positioniert ist, mit einer numerischen Apertur (NA von 0,4 in die Probenebene projiziert. Die mittels Elektronenstrahl-Lithographie gefertigte Maske besteht aus einem transparenten Calciumfluorid-Substrat $\left(\mathrm{CaF}_{2}\right)$, auf dessen Oberfläche eine eindimensionale Gitterstruktur aus Chrom gefertigt ist (Transmissionsgitter). Mithilfe des 
SCHWARZSCHILD-Objektivs wird nun die Gitterperiode der Maske von rund $20 \mu \mathrm{m}$ um den Faktor 25 verkleinert, so dass eine Gitterstruktur mit einer Periode von annähernd $800 \mathrm{~nm}$ auf der $\mathrm{Nb}_{2} \mathrm{O}_{5}$-Oberfläche entsteht. Bei vollständiger Ausleuchtung der Maske $\left(5 \times 5 \mathrm{~mm}^{2}\right)$ beträgt die räumliche Ausdehnung des erzeugten Oberflächengitters $200 \times 200 \mu^{2}$. Zur Bestimmung der Energie in der Maskenebene wird ein pyroelektrischer Energiedetektor vom Typ Molectron J50LP-4 (COHERENT) eingesetzt, welcher eine absolute Genauigkeit von etwa $20 \%$ besitzt.

Bei der anschließenden Auswertung aller auf der $\mathrm{Nb}_{2} \mathrm{O}_{5}$-Schicht erzeugten Gitter wird das geeignete Prozessfenster für die $\mathrm{F}_{2}$-Laserablation bestimmt: Bei Energiedichten (Fluenzen) im Bereich von $260 \mathrm{~mJ} / \mathrm{cm}^{2}$ bis knapp $350 \mathrm{~mJ} / \mathrm{cm}^{2}$ findet ein Materialabtrag an der Oberfläche der $\mathrm{Nb}_{2} \mathrm{O}_{5}$-Proben statt. Damit sind

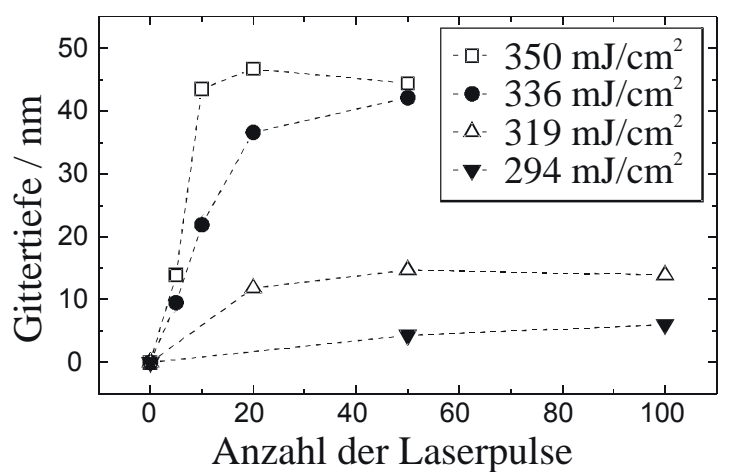

Abb. 3.3: Mittels $\mathrm{F}_{2}$-Laserablation erzeugte mittlere Gitteramplituden/Gittertiefen über der Pulszahl für verschiedene Fluenzen. Absorptionseigenschaften des Materials der Bearbeitung von $\mathrm{Nb}_{2} \mathrm{O}_{5}$ enge Grenzen gesetzt. Bereits ab einer Fluenz von $350 \mathrm{~mJ} / \mathrm{cm}^{2}$ ist die Zerstörschwelle, d.h. die Aufösung der Gitterstruktur, erreicht. Da jedoch unterhalb von $260 \mathrm{~mJ} / \mathrm{cm}^{2}$ kein Materialabtrag verzeichnet werden kann, markiert dies gleichzeitig die Ablationsschwelle des Materials. Wie die Auswertungen mit einem Rasterkraftmikroskop (RKM) gezeigt haben, besitzen die nahe an der Ablationsschwelle erzeugten Gitter ein annähernd trapez-förmiges Gitterprofil, dessen Oberfläche aber sehr rau und uneben ist. Ein nahezu vollständig homogenes und sinusförmiges Gitterprofil konnte hingegen bei Fluenzen nahe am oberen Ende des Prozessfensters erreicht werden (BADER u. a. 2004).

In Abb. 3.3 sind die mittels $\mathrm{F}_{2}$-Laserablation erzeugten mittleren Gitteramplituden (Gittertiefen) über der Pulszahl für verschiedene Fluenzen dargestellt. Deutlich ist der schnelle Anstieg der Gitteramplitude bei wachsender Pulszahl besonders für die Fluenzen $336 \mathrm{~mJ} / \mathrm{cm}^{2}$ und $350 \mathrm{~mJ} / \mathrm{cm}^{2}$ zu erkennen. Mit zunehmender Puls- 
zahl ist bei allen Fluenzen ein sättigendes Verhalten in Form eines Plateaus zu erkennen, das für jede Fluenz zwar unterschiedlich schnell, spätestens jedoch ab 50 Pulsen, erreicht wird. Bei der Bestrahlung der Proben mit maximaler Fluenz von $350 \mathrm{~mJ} / \mathrm{cm}^{2}$ ist in Abb. 3.3 auch die maximal erreichbare Gittertiefe von $50 \mathrm{~nm}$ zu erkennen. Als mögliche Ursache für die Limitierung der Modulationstiefe scheint die bei hohen Pulszahlen entstehende Schmelzdynamik an der $\mathrm{Nb}_{2} \mathrm{O}_{5}$-Oberfläche denkbar (BADER u. a. 2006).

\subsection{Fertigung wellenleitender Schichten}

Mit der Beschichtung vorstrukturierter AF 45- und D263 T bzw. unstrukturierter Quarzglas-Substrate mit einem optisch hochbrechenden Material bekommt die DGWS bzw. GWS ihre wellenleitende Eigenschaft. Um jedoch eine optimale Haftung des Wellenleiters auf der Substratoberfläche zu gewährleisten, erscheint der Einsatz von Wellenleitermaterialien, die im Vergleich zu den verwendeten Substratmaterialen ähnliche physikalisch-chemische Eigenschaften besitzen, sinnvoll. Die im Rahmen dieser Arbeit verwendeten Wellenleitermaterialien Tantalpentoxid $\left(\mathrm{Ta}_{2} \mathrm{O}_{5}\right)$ sowie die bereits in Abschnitt 3.2 .2 erwähnten $\mathrm{Nb}_{2} \mathrm{O}_{5}$ und Hafniumoxid $\left(\mathrm{HfO}_{2}\right)$ zeichnen sich durch ihre hervorragenden optischen bzw. wellenleitenden Eigenschaften aus. Diese stellen sie bereits seit vielen Jahrzehnten in der integrierten Optik erfolgreich unter Beweis (EsCOUBAS und FLORY 2003).

Für die Beschichtung von Substraten mit Metalloxiden haben sich je nach Anspruch an die herzustellende Schicht verschiedene Verfahren etabliert. Das sog. Abscheiden optisch dünner Schichten wird durch das Verdampfen oder Zerstäuben eines teilweise in einer Hochvakuum-Beschichtungsanlage befindlichen Zielmaterials (engl. target) erreicht und allgemein als physikalische Gasphasenabscheidung (engl. physical vapor deposition, kurz PVD bezeichnet. Durch den innerhalb der Beschichtungsanlage befindlichen Unterdruck fällt das Target-Material bei diesem Depositionsverfahren als Niederschlag auf das Trägersubstrat. Das Schichtwachstum wird dabei über einen sog. Schwingquarz kontrolliert, welcher aufgrund seiner Massenzunahme während der Beschichtung seine Schwingfrequenz verändert. Zusätzlich befindet sich das Substrat i.d. R. auf einer rotierenden Kalotte, die für eine gleichmäßige Beschichtung sorgt. 
Ein zur Beschichtung der Probe DGWS/Hf331/18 genutztes Verdampfungsverfahren stellt das sog. Elektronenstrahlverdampfen (engl. electron-beam evaporation -EBE dar, welches im Zusammenhang mit der PVD auch als EBPVD bezeichnet wird. Da zur Fertigung der Wellenleiterschicht eine vergleichsweise geringe kinetische Energie verwendet wird, bildet sich innerhalb der Schicht eine Säulenstruktur aus (GUENTHER 1982), was in der Folge zu einer geringen Packungsdichte (etwa $90 \%)$ bzw. einer vergleichsweise porösen Materialstruktur der $\mathrm{HfO}_{2}$-Schicht führt (EBERT und EBERT 2002). Dies ermöglicht in Abhängigkeit von der Umgebungstemperatur die Einlagerung von Wasser (chemische Absorption) aus der umgebenden Luft und macht somit auch den (mittleren) Brechungsindex der $\mathrm{HfO}_{2}$-Schicht temperaturabhängig. Die Charakterisierung der Probe DGWS/Hf331/18 findet in Abschnitt 4.2.3.2 statt.

Durch die Verwendung einer zusätzlichen Plasma-Ionenquelle ist es in Zusammenhang mit der PVD möglich, die Packungsdichte der Metalloxidschichten zu erhöhen und damit die Porosität der Schichten auf unter $3 \%$ zu senken (EBERT und EBERT 2002). Dieses Depositionsverfahren wird auch plasma-ionengestütztes Aufdampfen (engl. plasma-ion-assisted deposition - PIAD) genannt. Alle DWG-Proben, deren Wellenleiterschicht aus $\mathrm{Nb}_{2} \mathrm{O}_{5}$ oder $\mathrm{HfO}_{2}$ gefertigt ist, sind mittels EBPVD oder PIAD in einer Hochvakuumbeschichtungsanlage vom Typ BALzERs BAK 640 bzw. LEYBOLd SYRUSpro 1100 am Fraunhofer-Institut FÜr AngeWANDTE OpTIK UND FEINMECHANIK (IOF) hergestellt worden (HEGER 2005).

Ein weiteres und zudem sehr verbreitetes PVD-Verfahren stellt das sog. Kathodenzerstäuben (Sputtern) dar, bei dem kleinste - meist elektrisch geladene - Teilchen aus dem Material (Target) herausgelöst und durch eine angelegte Spannung zwischen Target (Kathode) und Substrat (Anode) auf der Substratoberfläche deponiert werden. Beispielsweise werden beim sog. Plasma- oder Gasentladungssputtern mithilfe einer Gasentladung (Plasma) Argon-Ionen $\left(\mathrm{Ar}^{+}\right)$erzeugt, welche anschließend in einem elektrischen Hochspannungsfeld auf das Target beschleunigt werden. Aufgrund der hohen kinetischen Energie der $\mathrm{Ar}^{+}$von typischerweise 30$50 \mathrm{eV}$ lösen sie Teilchen aus dem Material heraus, die im Anschluss auf dem Substrat abgeschieden werden. Dieses Verfahren bietet i. d. R. eine hohe Haftfestigkeit der Schicht auf dem Trägersubstrat sowie eine geringe thermische Belastung während des Prozesses (PULKER 2003). 
Beim sog. Puls-Magnetron-Sputtern (PMS) wird wie bei der PIAD zusätzlich zum elektrischen Feld ein Magnetfeld erzeugt, welches für eine hohe Packungsdichte während der Beschichtung sorgt und deshalb eine im Vergleich zur EBPVD weniger poröse Schicht zur Folge hat. Die wellenleitende $\mathrm{Ta}_{2} \mathrm{O}_{5}$-Schicht der Probe DGWS/Ta150/24 bzw. DGWS/Ta150/40 wird mit einer speziellen reaktiven Form des PMS gefertigt (COTTIER u. a. 2002).

\subsection{Oberflächencharakterisierung gefertigter Wellenleitergitter}

Um das Resonanzverhalten der gefertigten DWG Proben besser zu verstehen, ist es notwendig, möglichst viele Strukturinformationen über die einzelnen Proben zu erhalten. Dies gelingt z. B. mithilfe des Rasterkraftmikroskops (RKM) und des Rasterelektronenmikroskops (REM), die es ermöglichen, die Oberflächenstruktur der gefertigten Proben mithilfe von Elektronen abzubilden und somit wichtige Daten über die Gitterstruktur, wie Periodizitäten oder Modulationen, aber auch Unregelmäßigkeiten innerhalb der Oberflächenstruktur zu erfassen.

Beim RKM wird die Oberfläche der Probe mithilfe einer mikroskopisch kleinen Spitze, die am Ende einer Blattfeder (Cantilever) sitzt, mechanisch abgetastet. Dazu wird die Messspitze zunächst mittels Piezostellelementen in einem geringen Abstand zur Probenoberfläche gebracht und anschließend in einem definierten Raster über die Oberfläche geführt. Aufgrund der zwischen der Cantilever und der Oberfläche wirkenden atomaren Kräfte erfährt die Cantilever eine Auslenkung, die mithilfe eines Laserstrahls und einem Sensor elektronisch erfasst wird. Aus den elektronischen Daten kann nun eine zwei- oder dreidimensionale Abbildung der Probenoberfläche erstellt werden. Mit einer geeigneten Messspitze können dabei noch Strukturen im ÅnGSTRÖM-Bereich aufgelöst werden.

Anders das REM, Die hochaufösenden Elektronenbilder hoher Schärfentiefe erlauben es, bei kleiner Vergrößerung einen makroskopischen Überblick über die Probenoberflächen zu geben, aber auch Bilder stark vergrößerter Probenausschnitte zu erzeugen. Zur Erfassung der Oberflächenstruktur wird beim REM ein fein gebündelter Elektronenstrahl (berührungslos) im Vakuum über die Oberfläche geführt. 


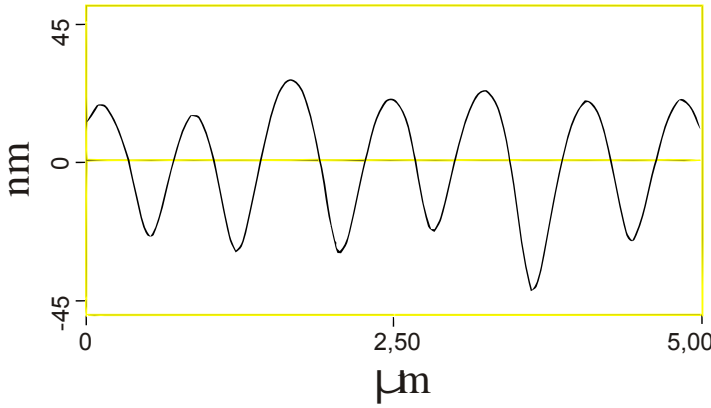

(a) Oberflächenprofil in der Mitte der strukturierten Probenoberfläche.

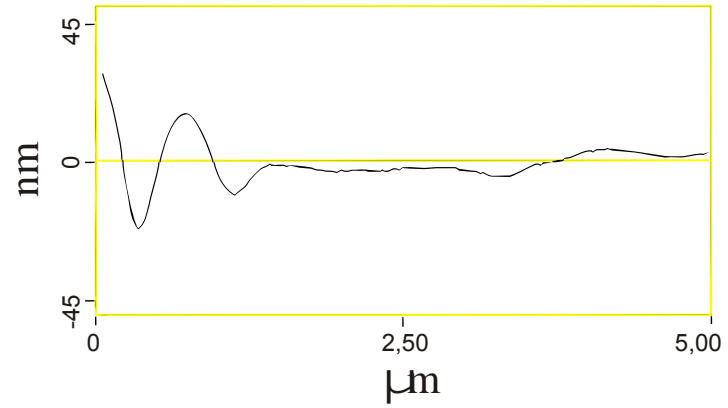

(b) Oberflächenprofil am Rand der strukturierten Probenoberfläche.

Abb. 3.4: Mit einem RKM aufgenommene Oberflächenprofile der ablatierten $\mathrm{Nb}_{2} \mathrm{O}_{5}$-Oberfläche (a) in der Mitte und (b) am Rand der Probe GWS/Nb150/42.

Durch die Wechselwirkungen der Elektronen mit den Atomen auf der Probenoberfläche werden die Elektronen abgelenkt oder rückgestreut. Da Elektronen eine elektrische Ladung besitzen, können die entstehenden Teilstrahlen - ähnlich dem Lichtmikroskop - mittels Elektronenlinsen gebündelt und detektiert werden. Um eine möglichst gute Wechselwirkung der Elektronen mit der Probe zu erreichen, wird die Leitfähigkeit der Oberfläche - i.d. R. durch die Bedampfung mit Gold erhöht. Viele Proben sind nach einer Bedampfung jedoch unbrauchbar, was besonders bei der Verwendung biologischer Proben einen Nachteil gegenüber dem RKM darstellt. Bedingt durch die im Vergleich zum sichtbaren Licht sehr viel kleinere Wellenlänge der Elektronenstrahlung kann das REM noch Strukturen im Nanometerbereich aufösen, erfasst im Gegensatz zum RKM jedoch keine Höheninformation der Probenoberfläche.

Abb. 3.4a zeigt das mit einem RKM aufgenommene Oberflächenprofil der Probe GWS/Nb150/42, deren $\mathrm{Nb}_{2} \mathrm{O}_{5}$-Oberfläche mithilfe der $\mathrm{F}_{2}$-Laserablation strukturiert worden ist (s. Abschnitt 3.2.2). Das sinusförmige Gitterprofil ist in einem $5 \mu \mathrm{m}$ langen Profilausschnitt dargestellt, der senkrecht zu den Gitterstegen verläuft. Durch wiederholte RKM-Aufnahmen an verschiedenen Stellen auf der Probenoberfläche kann die mittlere Gitteramplitude der Probe auf $42 \mathrm{~nm}$ bestimmt werden, wobei die maximalen Abweichungen vom Mittelwert ca. 25\% betragen. Die mittlere Periode des Gitters beträgt rund $780 \mathrm{~nm}$. 
Darüber hinaus zeigt Abb. 3.4b eine RKM-Aufnahme, die den Übergang vom strukturierten bzw. belichteten zum unstrukturierten bzw. unbelichteten Bereich der $\mathrm{Nb}_{2} \mathrm{O}_{5}$-Oberfläche wiedergibt. Der dargestellte Verlauf des Gitterprofils entspricht dabei der Form einer gedämpften Sinuswelle, deren mittlere Höhe nahezu gleich auf mit der Höhe des unstrukturierten Bereichs liegt. Damit kann nicht abschließend geklärt werden, ob bei der Strukturierung der $\mathrm{Nb}_{2} \mathrm{O}_{5}$-Oberfläche tatsächlich ein Materialabtrag stattgefunden hat. Geht man lediglich von einer Aufschmelzung der Materialoberfläche aus, bleibt die ursprüngliche Wellenleiterdicke von $150 \mathrm{~nm}$ auch nach der $\mathrm{F}_{2}$-Laserbearbeitung effektiv erhalten.

Ergänzend zu den RKM-Daten der Probe GWS/Nb150/42 ist in Abb. 3.5 eine REM-Aufnahme der bearbeiteten $\mathrm{Nb}_{2} \mathrm{O}_{5}$-Oberfläche abgebildet. Das kontrastreiche Bild zeigt einen ca. $60 \times 55 \mathrm{\mu m}^{2}$ großen Ausschnitt der Probenoberfläche unter einem Blickwinkel von etwa $45^{\circ}$. Während die Gitterstruktur in der Periodizität weitgehend homogen erscheint, sind in einigen Bereichen der Struktur sichtbare Unregelmäßigkeiten (helle bzw. dunkle Bereiche), welche auf starke Abweichungen in der Modulationstiefe des Gitters zurückzuführen sind, zu erkennen. Dies unterstützt die Vermutung, dass es bei der

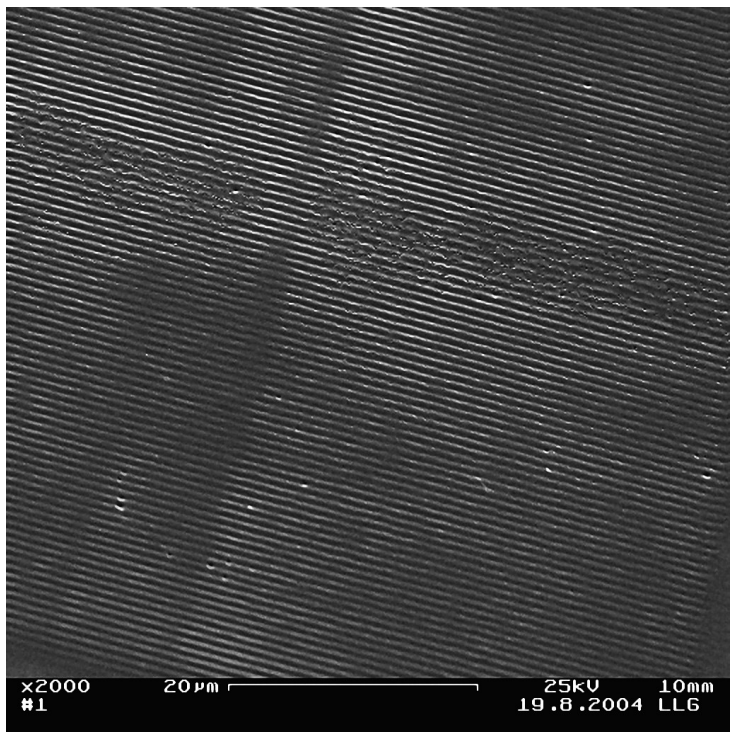

Abb. 3.5: REM-Aufnahme von der mittels $\mathrm{F}_{2}$-Laserablation strukturierten Oberfläche der Probe GWS/Nb150/42. resonanten Bestrahlung der Probe zu starken Streuverlusten an der Oberfläche der GWS kommt (s. Abschnitt 4.4).

Um das bei der Beschichtung erzeugte Oberflächengitter einer DGWS hinreichend interpretieren zu können, ist es vorteilhaft, neben der Oberflächencharakterisierung der gefertigten DGWS selbst auch das unbeschichtete Substratgitter zu untersuchen. Mit dem Vertrieb der vorstrukturierten Substrate durch die Firma OC Oerlikon Balzers ehemals Unaxis Balzers AG ist die Gitterstruktur der Substrate bereits in der Vergangenheit vom Hersteller umfassend charakterisiert 


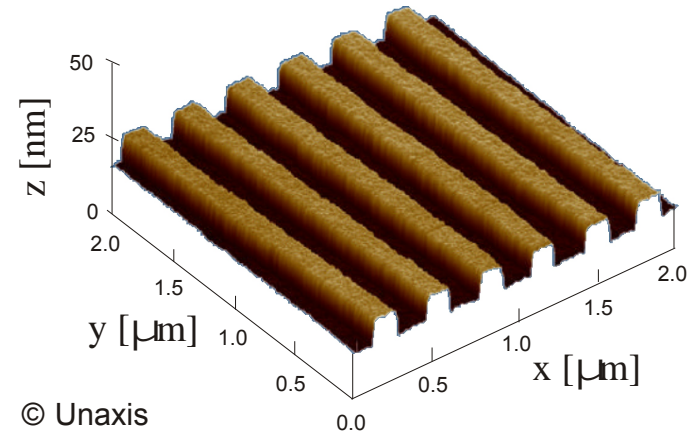

(a) Gitterstruktur eines unbeschichteten, vorstrukturierten Substrats.

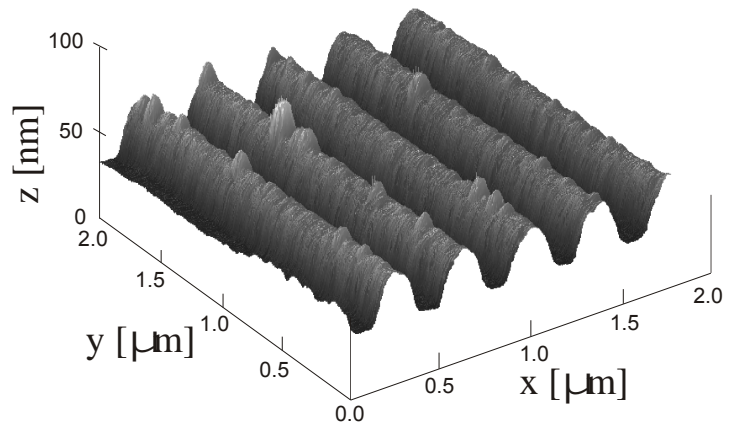

(b) Gitterstruktur nach der Beschichtung eines (vorstrukturierten) Substrats.

Abb. 3.6: Mit einem RKM aufgenommenes dreidimensionales $2 \times 2 \mu \mathrm{m}^{2}$ großes $\mathrm{Ab}-$ bild der Oberflächenstruktur: (a) zeigt die Gitterstruktur eines unbeschichteten Substrats mit einer Gitterperiode $318 \mathrm{~nm}$ und einer Gittertiefe $12 \mathrm{~nm}$ (Quelle: UnAXIs), (b) zeigt die Oberfläche der Probe DGWS/Ta150/24 nach der Beschichtung des Substrats.

worden. Abb. 3.6a zeigt die RKM-Aufnahme eines Substratgitters, das sich mit einer Gitterperiode von $318 \mathrm{~nm}$ und einer Gittertiefe von $12 \mathrm{~nm}$ zwar deutlich von den Gitterstrukturdaten der im Rahmen dieser Arbeit verwendeten Substratgitter unterscheidet, jedoch dieselben Qualitätsmerkmale besitzt. Die in dem $2 \times 2 \mu^{2}$ großen Ausschnitt gezeigte Gitterstruktur besitzt augenscheinlich eine hochgradig periodische Struktur und zeigt darüber hinaus das für den photolithographischen Herstellungsprozess typische Rechteckprofil mit einer durchweg hohen Flankensteilheit in nahezu perfekter Form (s. Abschnitt 3.2.1). Das Tastverhältnis (auch Füllfaktor genannt), welches aus dem Verhältnis von Gittersteg und -periode gebildet wird, beträgt annähernd 0,5. Zudem besitzt die gesamte Struktur eine sehr niedrige Oberflächenrauigkeit, die auf wenige Nanometer geschätzt wird.

Um die Qualität der nach einer Beschichtung erzeugten Oberflächengitter zu überprüfen, sind am Beispiel der Probe DGWS/Ta150/24 eigene Oberflächenuntersuchungen mithilfe des RKM durchgeführt worden. Abb. $3.6 \mathrm{~b}$ zeigt einen $2 \times 2 \mathrm{\mu m}^{2}$ großen Ausschnitt der Probenoberfläche mit einer deutlich sichtbaren Gitterstruktur. Mithilfe des RKM konnten die vom Hersteller angegebenen Strukturdaten zur Periode $(360 \mathrm{~nm})$ und Gittertiefe $(24 \mathrm{~nm})$ im Mittel mit einer maximalen 
Abweichung von $10 \%$ bestätigt werden. Auffälligstes Unterscheidungsmerkmal der beschichteten DGWS-Probe im Vergleich zum unbeschichteten Substrat (vgl. Abb. 3.6a) ist das veränderte Gitterprofil, was auf ein inhomogenes Schichtwachstum während des Beschichtungsprozesses zurückzuführen ist. So zeigt das Oberflächengitter, welches nach dem Aufbringen der $150 \mathrm{~nm}$ dicken $\mathrm{Ta}_{2} \mathrm{O}_{5}$-Schicht entstanden ist, ein eher sinus- als rechteckförmiges Gitterprofil. Wie zuvor beim Substratgitter beträgt das Tastverhältnis des Oberflächengitters annähernd 0,5.

Zudem ist die Rauigkeit der in Abb. 3.6b gezeigten Oberfläche gegenüber der unbeschichteten Substratprobe in Abb. 3.6a leicht erhöht. Dies hängt, wie die Erfahrungen bei der Beschichtung planarer Wellenleiter gezeigt haben, jedoch stark von dem jeweiligen Beschichtungsprozess ab und ist zudem schwer von möglichen Verunreinigungen auf der Probenoberfläche unterscheidbar.

Darüber hinaus kann es bei der Herstellung verhältnismäßig (im Vergleich zur Gitterperiode) dicker Wellenleiterschichten innerhalb eines nicht vollständig homogenen Beschichtungsprozesses zu einem lateralen Versatz zwischen Substratund Oberflächengitter kommen. Dieser Phasenversatz zwischen beiden Gittern beeinflusst das Resonanzverhalten und wird darüber hinaus am Beispiel der Proben DGWS/Hf331/18 und DGWS/Nb487/40 (s. Abschnitt 4.2.3.2 bzw. 4.2.3 als Erklärung für das abweichende Verhalten der Proben bei resonanter Bestrahlung benutzt. Eine genauere Untersuchung der Probenstrukturen im Hinblick auf einen möglichen relativen Gitterphasenversatz war im Rahmen dieser Arbeit jedoch nicht möglich.

\subsection{Zusammenfassung der Strukturdaten gefertigter Wellenleitergitter}

Im Rahmen dieser Arbeit sind insgesamt neun verschiedene DWG-Proben (acht DGWS und eine GWS angefertigt worden, deren Strukturdaten in diesem Abschnitt zusammengefasst sind. In Tab. 3.1 sind dazu sämtliche Strukturdaten aller verwendeten DWG-Proben übersichtlich dargestellt: Neben den Gitterstrukturdaten Periode und Tiefe können auch Wellenleitermaterial und -dicke sowie das 


\begin{tabular}{|c|c|c|c|c|c|}
\hline \multirow[b]{2}{*}{ Probenbezeichnung } & \multicolumn{2}{|c|}{ Gitter- } & \multicolumn{2}{|c|}{ Wellenleiter- } & \multirow{2}{*}{$\frac{\text { Substrat- }}{\text { Material }}$} \\
\hline & Periode/nm & Tiefe/nm & Material & Dicke/nm & \\
\hline DGWS/Hf30/18 & 360 & 18 & $\mathrm{HfO}_{2}$ & 30 & AF 45 \\
\hline DGWS/Hf30/40 & 360 & 40 & $\mathrm{HfO}_{2}$ & 30 & D $263 \mathrm{~T}$ \\
\hline DGWS/Hf74/40 & 360 & 40 & $\mathrm{HfO}_{2}$ & 74 & D $263 \mathrm{~T}$ \\
\hline DGWS/Hf331/18 & 360 & 18 & $\mathrm{HfO}_{2}{ }^{*}$ & 331 & AF 45 \\
\hline DGWS/Nb487/40 & 360 & 40 & $\mathrm{Nb}_{2} \mathrm{O}_{5}$ & 487 & D $263 \mathrm{~T}$ \\
\hline DGWS/Nb551/40 & 360 & 40 & $\mathrm{Nb}_{2} \mathrm{O}_{5}$ & 551 & D $263 \mathrm{~T}$ \\
\hline DGWS/Ta150/24 & 360 & 24 & $\mathrm{Ta}_{2} \mathrm{O}_{5}$ & 150 & AF 45 \\
\hline DGWS/Ta150/40 & 360 & 40 & $\mathrm{Ta}_{2} \mathrm{O}_{5}$ & 150 & D $263 \mathrm{~T}$ \\
\hline GWS/Nb150/42 & 780 & 42 & $\mathrm{Nb}_{2} \mathrm{O}_{5}$ & 150 & Quarzglas \\
\hline
\end{tabular}

Tab. 3.1: Übersicht zu den Strukturdaten aller verwendeten DWG-Proben. Die angegebene Wellenleiterdicke entspricht der Beschichtungsdicke und unterscheidet sich daher von

verwendete Substratmaterial der jeweiligen Probe zugeordnet werden. Die individuelle Bezeichnung der Proben, welche im Rahmen dieser Arbeit durchweg benutzt wird, setzt sich aus der Strukturform, des abgekürzten Wellenleitermaterials und -dicke sowie aus der Gittertiefe zusammen und ist für jede Probe eindeutig.

Mit Ausnahme der Probe GWS/Nb150/42, bei der das Substratmaterial aus Quarzglas besteht, wird bei den übrigen Proben eines der SchotT ${ }^{\circledR}$ _Gläser AF 45 (Dicke 0,7 mm) bzw. D $263 \mathrm{~T}$ (Dicke 1,1 mm) verwendet. Die Abmessungen der flachen Seite betragen beim AF 45-Substrat $1,6 \mathrm{~cm} \times 4,8 \mathrm{~cm}$ bzw. beim D 263 T-Substrat $2,5 \mathrm{~cm} \times 7,6 \mathrm{~cm}$. Das vom Hersteller angegebene Transmissionsverhalten der Gläser ist in den angehängten Abb. A.1 bzw. A.2 einsehbar.

Wie die Tabelle weiterhin zeigt, kennzeichnet alle DGWS-Proben die gleiche Gitterperiode von $360 \mathrm{~nm}$. Darüber hinaus werden diese Proben durch ihre Gittertiefe, die wiederum an das Substratmaterial geknüpft ist, bzw. durch die Abmessungen ihrer Gitterstruktur auf der Substratoberfläche unterschieden: Während bei den $18 \mathrm{~nm}$ tiefen Gittern eine Seite der Gitterfläche entlang der langen Substratseite auf ca. $0,5 \mathrm{~mm}$ begrenzt ist (Wellenleiter-Koppelgitter), erstreckt sich die $40 \mathrm{~nm}$ tiefe Gitterstruktur über die gesamte D 263 T-Substratfläche. Ohne dass es einer

\footnotetext{
*mittels EBPVD gefertigte poröse $\mathrm{HfO}_{2}$-Schicht.
} 
Oberflächencharakterisierung jeder einzelnen DGWS-Probe bedarf, ist davon auszugehen, dass die industriell gefertigten Substratgitter bei allen DGWS-Proben ein nahezu rechteckiges Gitterprofil besitzen, dessen Tastverhältnis etwa 0,5 beträgt. Wie jedoch der Abschnitt 3.4 gezeigt hat, wird die Profilinformation mit der Beschichtung der Substratgitter nicht an das Oberflächengitter übertragen, so dass bei allen DGWS-Proben eher von einem Sinus- als einem Rechteckprofil auszugehen ist.

In unterschiedlichen Dicken von 30-551 nm fanden zudem die Materialien $\mathrm{Ta}_{2} \mathrm{O}_{5}$, $\mathrm{Nb}_{2} \mathrm{O}_{5}$ und $\mathrm{HfO}_{2}$ als Wellenleiter der jeweiligen Proben Verwendung. Insbesondere die Wellenleiterschicht der Probe DGWS/Hf30/40 mit einer Beschichtungsdicke von $30 \mathrm{~nm}$ im Vergleich zur Gittertiefe $(40 \mathrm{~nm})$ suggeriert hier eine nicht durchgehende anisotrope Schicht, welche jedoch im Rahmen dieser Arbeit nicht näher untersucht wird. Dass trotz dieser mutmaßlich periodisch unterbrochenen (fraktionierten) Schicht eine resonante Anregung der Probe dennoch möglich ist, stellt zum einen die Charakterisierung der DGWS bei der Laserwellenlänge $266 \mathrm{~nm}$ in Abschnitt 4.2.1 unter Beweis und wird zum anderen von numerischen Simulationen RCWA gestützt.

Aufgrund der mittels EBPVD erzeugten $\mathrm{HfO}_{2}$-Schicht besitzt darüber hinaus die Probe DGWS/Hf331/18 einen neuartigen Freiheitsgrad, der das Resonanzverhalten der Probe von der Umgebungstemperatur abhängig macht. Wie bereits in Abschnitt 3.1 erwähnt wird, ist es mit der porösen Wellenleiterschicht dieser Probe möglich, Wasser aus der Umgebung einzulagern (offene Porosität) und den Wassergehalt der Schicht über die Umgebungstemperatur zu beeinflussen. Aufgrund des höheren Brechungsindexes des Wassers $(1,33)$ im Vergleich zur umgebenden Luft $(1,000)$ variiert je nach Wassergehalt auch der mittlere Brechungsindex der Schicht und ermöglicht dadurch eine Verschiebung der Resonanz (s. Abschnitt 2.4.2). Während alle übrigen Proben eine rein passive Struktur besitzen, stellt die Probe DGWS/Hf331/18 somit die einzige im Rahmen dieser Arbeit verwendete DWG-Probe dar, die ein durchstimmbares Resonanzverhalten zeigt (s. Abschnitt 4.2.3.2. 


\section{Optische Charakterisierung resonanter Wellenleitergitter}

Das Kapitel befasst sich mit der optischen Charakterisierung resonanter Wellenleitergitter und gliedert sich wie folgt: Nach einem kurzen tabellarischen Überblick über die zu charakterisierenden DWG Proben widmet sich Abschnitt 4.1 der experimentellen Bestimmung von Resonanzkennlinien. Im Anschluss beschäftigt sich Abschnitt 4.2 mit der Charakterisierung von DWG Proben, deren resonantes Transmissions- bzw. Reflexionsverhalten in verschiedenen Messanordnungen mithilfe von Laserlicht bestimmt wird. Dazu wird zunächst das Resonanzverhalten der Proben DGWS/Hf30/40, DGWS/Hf30/18 und DGWS/Hf74/40 bei den UV-Wellenlängen 266,0 nm und 354,7 nm mit einem Reflektometer goniometrisch untersucht. In Abschnitt 4.2.2 werden die Proben DGWS/Ta150/24, DGWS/Ta150/40 sowie GWS/Nb150/42 durch die Zentralwellenlänge $800 \mathrm{~nm}$ eines breitbandigen Titan:Saphir-Laserspektrums in Resonanz versetzt und deren Transmissions- bzw. Reflexionsspektrum bei festem Einfallswinkel mit einem Spektrograph erfasst. Mit einem schmalbandigen und durchstimmbaren Farbstofflaser wird in Abschnitt 4.2.3 das Resonanzverhalten der Proben DGWS/Nb487/40, DGWS/Nb551/40 und DGWS/Hf331/18 untersucht, wobei das temperaturabhängige Verhalten der Probe DGWS/Hf331/18 in Abschnitt 4.2.3.2 gesondert charakterisiert wird. Die Transmissions- und Reflexionsmessungen enden mit Abschnitt 4.4 . in welchem die Ergebnisse überblickend dargestellt und diskutiert werden. Zum Abschluss dieses Kapitels beschäftigt sich Abschnitt 4.3 mit der zeitlichen Pulslänge resonant reflektierter Laserpulse.

Um eine Abschätzung über das zu erwartende Resonanzverhalten aller in diesem Kapitel charakterisierten DWG-Proben zu bekommen, sind in Tab. 4.1 für jede Probe die angestrebte Resonanzwellenlänge mit zugehöriger Laserstrahlquelle und Polarisation dem berechneten bzw. angestrebten Resonanzwinkel gegenübergestellt. Aufgrund ungenauer Brechzahldaten der Wellenleitermaterialien sind die 


\begin{tabular}{|c|c|c|c|c|}
\hline \multirow[b]{2}{*}{ Probenbezeichnung } & \multicolumn{2}{|c|}{ Laserstrahl- } & \multicolumn{2}{|c|}{ Resonanz- } \\
\hline & Quelle & Polarisation & Wellenlänge & Winkel \\
\hline DGWS/Hf30/40 & Nd:YAG-Laser* & TE & $266,0 \mathrm{~nm}$ & $\approx+45^{\circ}$ \\
\hline DGWS/Hf30/18 & Nd:YAG-Laser* & TE & $266,0 \mathrm{~nm}$ & $\approx+45^{\circ}$ \\
\hline DGWS/Hf74/40 & Nd:YAG-Laser** & $\mathrm{TE}$ & $354,7 \mathrm{~nm}$ & $\approx+45^{\circ}$ \\
\hline DGWS/Hf74/40 & Nd:YAG-Laser** & $\mathrm{TM}$ & $354,7 \mathrm{~nm}$ & $\approx+30^{\circ}$ \\
\hline DGWS/Hf331/18 & He-Ne-Laser ${ }^{\dagger}$ & TM & $632,816 \mathrm{~nm}$ & $\approx 0^{\circ}$ \\
\hline DGWS/Nb487/40 & Rubinlaser $^{\dagger}$ & $\mathrm{TE}$ & $694,3 \mathrm{~nm}$ & $\approx 0^{\circ}$ \\
\hline DGWS/Nb551/40 & Rubinlaser $^{\dagger}$ & $\mathrm{TM}$ & $694,3 \mathrm{~nm}$ & $\approx 0^{\circ}$ \\
\hline DGWS/Ta150/24 & Ti:Saphir-Laser & $\mathrm{TE}$ & $800 \mathrm{~nm}$ & $\approx-30^{\circ}$ \\
\hline DGWS/Ta150/24 & Ti:Saphir-Laser & TM & $800 \mathrm{~nm}$ & $\approx-45^{\circ}$ \\
\hline DGWS/Ta150/40 & Ti:Saphir-Laser & $\mathrm{TE}$ & $800 \mathrm{~nm}$ & $\approx-30^{\circ}$ \\
\hline DGWS/Ta150/40 & Ti:Saphir-Laser & TM & $800 \mathrm{~nm}$ & $\approx-45^{\circ}$ \\
\hline GWS/Nb150/42 & Ti:Saphir-Laser & TE & $800 \mathrm{~nm}$ & $\approx+45^{\circ}$ \\
\hline
\end{tabular}

Tab. 4.1: Probenübersicht mit (Design-)Wellenlänge und zugehörigem, angestrebtem Resonanzwinkel (Abschätzung) unter Angabe der Laserstrahlquelle und Polarisation.

Angaben zum (errechneten) Resonanzwinkel nur geschätzte Werte und weichen deshalb, wie Tab. 4.2 am Ende des Kapitels zeigt, vereinzelt deutlich von den experimentell bestimmten Werten ab. Darüber hinaus werden die in Tab. 4.1 angegebenen Laserstrahlquellen der Proben DGWS/Hf331/18 (Helium-Neon-Laser) und DGWS/Nb487/40 bzw. DGWS/Nb551/40 (Rubinlaser) durch einen durchstimmbaren Farbstofflaser ersetzt, wodurch die Möglichkeit der vollständigen spektralen Charakterisierung dieser Proben mit nahezu monochromatischem Licht gegeben ist (s. Abschnitt 4.2.3).

\subsection{Messung der Resonanzkennlinie}

Um die Resonanzkennlinie einer DWG experimentell zu bestimmen, wird am Beispiel der Probe DGWS/Ta150/40 zunächst das Transmissionsspektrum dieser

\footnotetext{
*4. Harmonische der Fundamentalwellenlänge 1064,14 nm.

**3. Harmonische der Fundamentalwellenlänge 1064,14 nm.

${ }^{\dagger}$ Ursprünglich geplante Laserquelle durch einen durchstimmbaren Farbstofflaser ersetzt.
} 
Probe in einem Spektrometer des Typs Lambda 19 der Firma PeRKINELmer erfasst. Die innerhalb des Spektrometers emittierende Weißlichtquelle (Gasentladungslampe) erzeugt dabei über einen weiten Spektralbereich (UV bis in IR) Licht, welches anschließend auf ein dispersives Beugungsgitter gelangt und dadurch spektral zerlegt wird. Nach Passieren eines Monochromators, der mithilfe von Spaltblenden die einzelnen Farben bzw. Wellenlängen des Spektrums selektiert, fällt das (monochromatische) Licht schließlich senkrecht auf die Probe. Über einen Photodetektor, der sich hinter der Probe befindet, wird das transmittierte Licht elektronisch erfasst und mithilfe eines Computerprogramms ausgewertet.

Um die Resonanzkennlinie der Probe DGWS/Ta150/40 zu bestimmen, muss zusätzlich zur Bestimmung der Resonanzwellenlänge über die Spektren (s. u.) auch der Einfallswinkel, d.h. der Winkel zwischen einfallendem Strahl und der Oberflächennormalen der Probe, ermittelt werden. Dazu wird die Probe auf einem Drehtisch montiert, der über eine goniometrische Skala die präzise Ausrichtung der Probe nach dem Nonius-Prinzip sicherstellt. Zur Stabilisierung des Drehtisches wird dieser auf einer massiven Metallplatte montiert, die innerhalb des Spektrometers so platziert ist, dass die Probe genau im optischen Strahlgang liegt. Bei der anschließenden Bestrahlung der Probe wird zu jedem Winkel die Wellenlänge des Spektrums 250-900 nm in Ein-Nanometer-Schritten angesteuert und die detektierte Intensität jeder Wellenlänge in eine Datenbank geschrieben. Bei einer Winkelschrittweite von $5^{\circ}$ und einem Winkelspektrum von $0-75^{\circ}$ werden somit insgesamt 16 Spektren aufgenommen (s. Abschnitt A Abb. A.4).

Laut Hersteller beträgt die Abweichung der vom Spektrometer angesteuerten Wellenlänge für diesen Spektralbereich unter $2 \AA$. Zudem kommt ein systematischer Fehler bei der Ausrichtung des Drehtisches hinzu, der in Zusammenhang mit dem Ablesefehler der NoniUs-Skala auf unter $\pm 0,2^{\circ}$ geschätzt wird. Die Ausrichtung der Probe wird jedoch von einer weiteren Fehlerquelle beeinflusst: Da bei jeder Neuausrichtung der Probe ein weiterer Ein- und Ausbau der Montageplatte und damit des Drehtisches notwendig ist, führt dies (zunächst) zu einem Gesamtfehler des Einfallswinkels von rund $\pm 0,5^{\circ}$.

Bei der anschließenden Auswertung der Transmissionsspektren werden die enthaltenen $T_{0}$-Minima, die einer resonanten Anregung der Probe unter dem jeweils eingestellten Winkel entsprechen, der Polarisation des einfallenden Lichts TE bzw. 


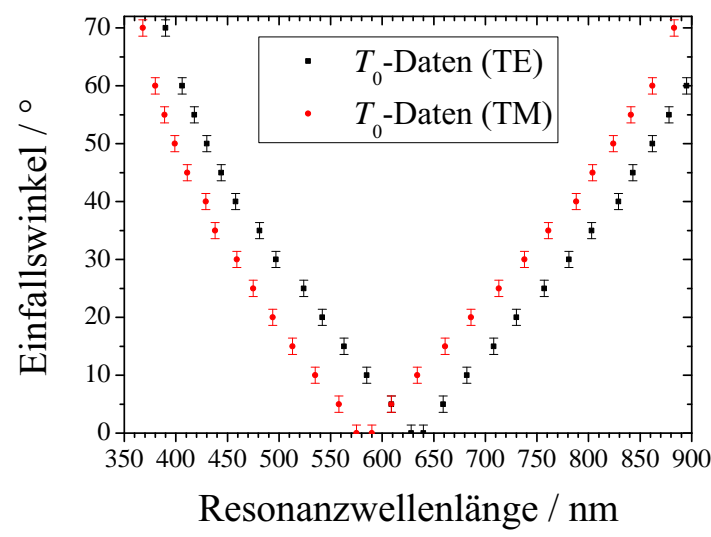

(a) Unkorrigierte (unvollständige) Resonanzkennlinien.

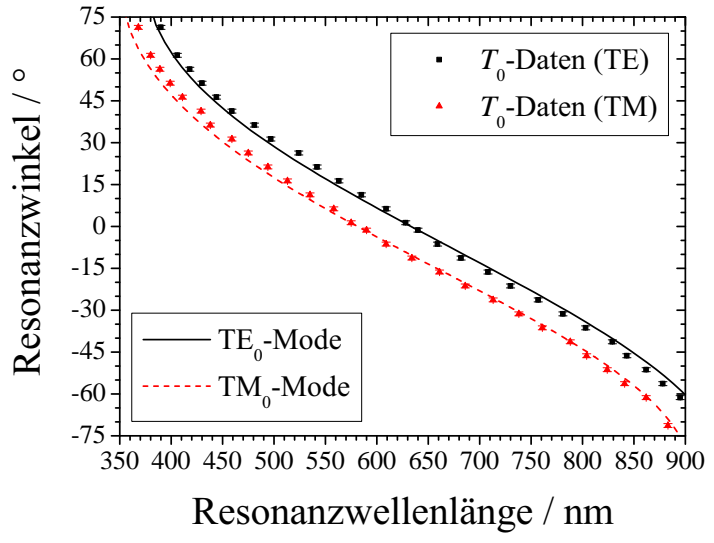

(b) Korrigierte, vollständige sowie berechnete (Mode) Resonanzkennlinien.

Abb. 4.1: Die nach Auswertung der Spektrometerdaten bestimmten $T_{0^{-}}$ Resonanzkennlinien der Probe DGWS/Ta150/40 für TE bzw. TM.Polarisation (a) mit Winkelversatz (unkorrigiert). (b) zeigt die korrigierten Messdaten aus (a) mit Richtungsinformation der einfallenden Welle (Vorzeichen) sowie die berechneten Resonanzkennlinien beider Polarisationen (Mode).

TM zugeordnet. Bedingt durch die zunehmende Absorption des Substratmaterials D 263 T für Wellenlängen nahe dem UV-Spektrum erfahren sämtliche Transmissionsspektren der Probe DGWS/Ta150/40 einen deutlichen Abfall in der Transmission für kleinere Wellenlängen ab ca. $350 \mathrm{~nm}$ (s. Abb. A.2). Die Auswertung der Transmissionspektren bezüglich höhermodiger Resonanzen, wie $\mathrm{TE}_{1}$ bzw. $\mathrm{TM}_{1}$ (s. Abb. 2.7), ist aufgrund der hohen Materialabsorption nicht möglich. Aufgrund der fehlenden Richtungsinformation der Propagationswelle geben die extrahierten $T_{0}$-Datensätze aus Einfallswinkel und Resonanzwellenlänge zunächst nur eine unvollständige Resonanzkennlinie wieder, was sich dadurch äußert, das jedem Einfallswinkel zwei Resonanzwellenlängen zugeordnet sind.

Abb. $4.1 \mathrm{a}$ zeigt die gemessenen Resonanzkennlinien der Probe DGWS/Ta150/40 für TE bzw. TM-Polarisation. Bei der Betrachtung des Einfallswinkels bei $0^{\circ}$ (Normalinzidenz) fällt auf, dass die Abszisse in Abhängigkeit von der jeweiligen Polarisation nicht (wie erwartet) einmal sondern zweimal geschnitten wird - nämlich bei den Resonanzwellenlängen $628 \mathrm{~nm}$ und $640 \mathrm{~nm}$ (TE) bzw. $575 \mathrm{~nm}$ und $590 \mathrm{~nm}$ (TM). Dieses Verhalten lässt nur den Schluss zu, dass der einfallende Lichtstrahl 
bei der Nullstellung der Winkelskala bei den Messungen nicht senkrecht zur Probe stand, sondern leicht versetzt gewesen sein muss. Unter Annahme eines (lokalen) linearen Verlaufs der Resonanzkennlinie mit den Resonanzwellenlängen $628 \mathrm{~nm}$ und $640 \mathrm{~nm}$ (TE) bzw. $575 \mathrm{~nm}$ und $590 \mathrm{~nm}(\mathrm{TM})$ als Kennlinien-Schnittpunkte beträgt dieser Winkelversatz für beide Polarisationen schätzungsweise 1,3 $\pm 0,2^{\circ}$. Der Gesamtfehler des Einfallswinkels, welcher bei den Messdaten in Abb. 4.1a in Form von Fehlerbalken enthalten ist, vergrößert sich damit auf $\pm 1,4^{\circ}$.

Abb. $4.1 \mathrm{~b}$ zeigt die korrigierten $T_{0}$-Resonanzkennlinien der Abb. 4.1a mit einem entsprechend angepassten Gesamtfehler (Fehlerbalken) bei nun rund $\pm 0,7^{\circ}$. Im Vergleich zu Abb. 4.1a enthält nun die Kennlinie auch die Richtungsinformation der Propagationswelle (vollständig), welche hier in Analogie zu Abschnitt 2.4.1 durch das entsprechende Winkelvorzeichen der einfallenden Welle dargestellt ist. Zusätzlich zu den Messdaten ist in Abb. 4.1b auch die berechnete Kennlinie der TE bzw. TM-Mode unter Berücksichtigung der Wellenlängendispersion von Substrat und Wellenleiter dargestellt. Zwar sind beide Kennlinien unter Berücksichtigung der Messgenauigkeit nicht deckungsgleich, jedoch geben die Messdaten den charakteristischen Kurvenverlauf von Resonanzwinkel- und wellenlänge perfekt wieder. Der etwas flachere Kurvenverlauf der berechneten Kennlinien geht mit großer Wahrscheinlichkeit auf die ungenaue Dispersionsfunktion der Wellenleiterbrechzahl zurück (keine Herstellerangaben verfügbar). Abgesehen von dieser Diskrepanz genügt die unter Abschnitt 2.4.1 vorgestellte analytische Betrachtung jedoch aus, die Charakteristik einer DWG-Kennlinie hinreichend zu beschreiben.

\subsection{Transmissions- und Reflexionsmessungen}

Zur weiteren Charakterisierung der Resonanz wird jede DWG-Probe mit dem Licht der jeweils angestrebten Resonanzwellenlänge bestrahlt und das gebeugte Licht nullter Ordnung in Transmission $\left(T_{0}\right)$ und Reflexion $\left(R_{0}\right)$ detektiert. $\mathrm{Zu}$ diesem Zweck werden unterschiedliche Messanordnungen verwendet, mit deren Hilfe die spektrale bzw. goniometrische Charakterisierung der verschiedenen Proben bei resonanter Anregung durchgeführt wird. Wichtigster Unterschied der einzelnen Messanordnungen ist die verwendete Laserstrahlquelle, die sich nach der anzuwendenden Resonanzwellenlänge richtet. 
Für die Charakterisierung der einzelnen Proben werden neben einem spektral durchstimmbaren Ti:Saphir- oder Farbstofflaser auch die 3. und 4. Harmonische eines Nd:YAG_Lasers mit den UVFWellenlängen 354,7 nm bzw. 266,0 nm eingesetzt. Während bei den Messungen mittels eines durchstimmbaren Lasers der Einfallswinkel fest vorgegeben ist, wird bei der Bestrahlung der Proben mit UV Laserlicht das Transmissions- bzw. Reflexionsverhalten der Proben goniometrisch bestimmt. Da bei allen Messungen evtl. auftretende optische Intensitätsverluste der einfallenden Laserstrahlung durch Materialabsorption $A$ oder Oberflächenstreuung $S$ nicht erfasst werden, können diese Größen nur als Summenbetrag $(A+S)$ innerhalb der (normierten) Intensitätsgleichung $T_{m}+R_{m}+(A+S)=1$ bestimmt werden, wobei $m$ die Beugungsordnung am Gitter angibt. Zudem treten bei den UVF Wellenlängen höhere Beugungsordnungen $(m>0)$ auf, die zwar bei der Auswertung bzw. Diskussion der Transmissions- und Reflexionsmessungen berücksichtigt, bei den Messungen selbst aber nicht detektiert werden.

\subsubsection{Goniometrische Bestimmung der Resonanz bei Anregung mit ultraviolettem Laserlicht}

Um das Resonanzverhalten der Proben DGWS/Hf30/40, DGWS/Hf30/18 und DGWS/Hf74/40 (s. Tab. 4.2 mit UV-Licht der Wellenlängen 354,7 nm bzw. $266,0 \mathrm{~nm}$ zu charakterisieren, wird die frequenzkonvertierte Infrarotstrahlung eines Nd:YAG Lasers verwendet. Die Frequenzkonversion der Fundamentalwellenlänge $1064,14 \mathrm{~nm}\left({ }^{4} F_{3 / 2} \rightarrow{ }^{4} I_{11 / 2}\right.$ Übergang $)$ des Lasers erfolgt mithilfe eines anisotropen Kristalls aus Beta-Bariumborat (BBO), dessen nichtlineare Eigenschaften die Erzeugung der 3. und 4. Harmonischen (354,7 nm bzw. 266,0 nm) der Fundamentalwellenlänge ermöglichen (LAUTERBORN und KURZ 2003; BOYD 2008).

Die Effizienz der Frequenzverdreifachung bzw. -vervierfachung hängt jedoch maßgeblich von der konstruktiven Überlagerung der Harmonischen (Oberwellen) mit der Fundamentalwellenlänge (Grundwelle) ab. So gelingt eine optimale Phasenanpassung (engl. index matching) nur dann, wenn die effektiven Brechzahlwerte des BBO-Kristalls für Grundwelle und Oberwelle gleich sind. Zudem sind die nichtlinearen Suszeptibilitäten im Vergleich zu den linearen meist einige Größenordnungen kleiner, so dass der Wirkungsgrad bei der Frequenzkonversion nur bei 
intensiver Bestrahlung des Kristalls, d.h. der Erzeugung hoher Energiedichten, möglich wird (YARIV und YEH 2003, EICHLER und EICHLER 2006).

In Abb. 4.2 ist die Messanordnung zur goniometrischen Charakterisierung der Proben DGWS/Hf30/40, DGWS/Hf30/18 und DGWS/Hf74/40 in $R_{0}$ und $T_{0}$ schematisch dargestellt. Der zur Bestrahlung der Proben verwendete gütegeschaltete Nd:YAG-Laser vom Typ PY61C-10 der Firma ContinuUM arbeitet bei einer Repetitionsrate von $10 \mathrm{~Hz}$. Bei einer mittleren Pulsenergie der Fundamentalwelle von etwa $75 \mathrm{~mJ}$ beträgt die Pulsdauer der Laserpulse etwa 40 ps. Nach der Frequenzkonversion durch den BBO-Kristall liegen die mittleren Ausgangsenergien der 3. und

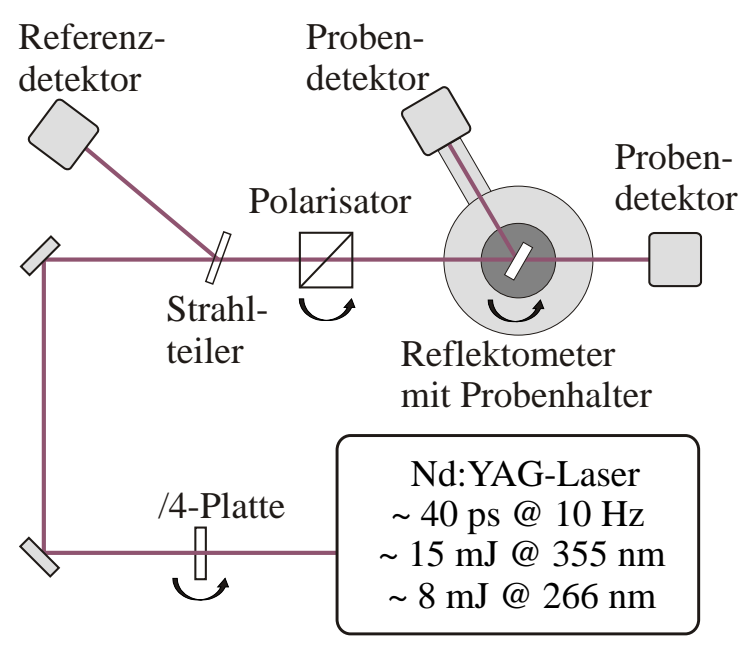

Abb. 4.2: Messanordnung zur goniometrischen Charakterisierung der Proben in Reflexion und Transmission.

4. Harmonischen bei rund $15 \mathrm{~mJ}$ bzw.

$8 \mathrm{~mJ}$ pro Laserpuls.

Mithilfe eines sog. Reflektometers ist eine vergleichsweise exakte goniometrische Vermessung der Proben in Transmissions- bzw. Reflexionsgeometrie möglich. Dazu wird der Laserstrahl über geeignete UV.Spiegel auf die zu charakterisierende Probe gelenkt, die im Zentrum des Reflektometers auf einem Halter montiert ist. Die Änderung des (Einfalls-)Winkels zwischen Probennormalen und einfallendem Laserstrahl wird dabei mithilfe eines Schrittmotors über ein Computerprogramm gesteuert. Die minimale Schrittweite des Einfallswinkels liegt bei etwa 5/1000 Grad. Bei der Nulldurchgangseichung der Reflektometerskala wird der von der Probe ausgehende Beugungsreflex (0. Ordnung) über alle optischen Komponenten zurück zum Laserausgang geführt und anschließend der eingestellte Winkel als Nullwert an die (Reflektometer)-Software übergeben. Der Fehler des Einfallswinkels wird somit auf unter $0,1^{\circ}$ geschätzt.

Die TE bzw. TM-Polarisation der einfallenden Strahlung wird über einen Polarisator festgelegt, der im Strahlengang direkt vor dem Reflektometer positioniert ist. 
Mithilfe einer $\lambda / 4$-Platte direkt am Laserausgang kann zudem die Intensität des einfallenden Lichts verändert werden. Die Energie, die von der Probenoberfläche reflektiert wird, wird mithilfe eines pyroelektrischen Energiemesskopfs (RjP-735, LASER PRECISION) detektiert, welcher das Messsignal in Reflexionsgeometrie ( $\theta$ 20-Konfiguration) aufnimmt. Zur Messung der Energie im transmittierten Strahl befindet sich hinter der Probe ein weiterer Detektor desselben Typs. Um das aufgezeichnete Messsignal normieren zu können, wird vor und nach jeder Messung die eingestrahlte Energie ohne Probe bestimmt. Um evtl. auftretende Intensitätsschwankungen oder Langzeitdrifteffekte des Lasers zu kompensieren, wird zudem während der Messungen ein Energiemesskopf vor dem Reflektometer (RjP-637, LAser Precision) positioniert. Dieser nimmt zeitgleich mit den anderen Messköpfen über einen Strahlteiler einen Teil der eingestrahlten Energie auf und dient bei der anschließenden Auswertung als Stabilitätsreferenz. Zudem wird bei der Bestrahlung der Probe DGWS/Hf30/18 eine Strahlreduzierungsoptik aus verschiedenen Linsen (Brennweiten $100 \mathrm{~mm}$ und 12,5 mm) eingesetzt, welche den Durchmesser des Laserstrahls $(\leq 0,4 \mathrm{~cm})$ etwa um den Faktor 8 verkleinert und somit die Bestrahlung des ca. 0,5 mm breiten Gitters für kleine Einfallswinkel mit einem annähernd kollimierten Strahl gerade noch möglich ist.

Abb. 4.3 zeigt die Ergebnisse der Reflexionsmessungen bei Bestrahlung der Proben DGWS/Hf30/40 und DGWS/Hf30/18 mit TE-polarisiertem Licht der UV Wellenlänge $266 \mathrm{~nm}$. Die Bestrahlung der Proben erfolgte von der Probenoberseite, d. h. dem Medienübergang Luft-Wellenleiter. Das aufgenommene Resonanzverhalten (Daten) ist dabei für beide Proben über einen Winkelbereich von 50-80 dargestellt. Bedingt durch die hohe Absorption der verwendeten Substratmaterialen D 263 T und AF 45 (s. Abb. A.1 und A.2 bei der Bestrahlung der Proben mit $266 \mathrm{~nm}$-Laserpulsen wird in Transmissionsgeometrie eine starke Fluoreszenzanregung beobachtet, wodurch die Intensität der Strahlung soweit abgeschwächt wird, dass eine Detektion des Transmissionssignals nicht mehr möglich ist. Über die in Abb. 4.3 gezeigten Reflexionsmaxima wird der Resonanzwinkel beider Proben bestimmt: Dieser liegt für die Probe DGWS/Hf30/40 bei einem Einfallswinkel von $65^{\circ}$ und für die Probe DGWS/Hf30/18 bei einem Winkel von $67^{\circ}$. Beide Winkel entsprechen damit nicht dem angestrebten Resonanzwinkel von $45^{\circ}$. Als Ursache 


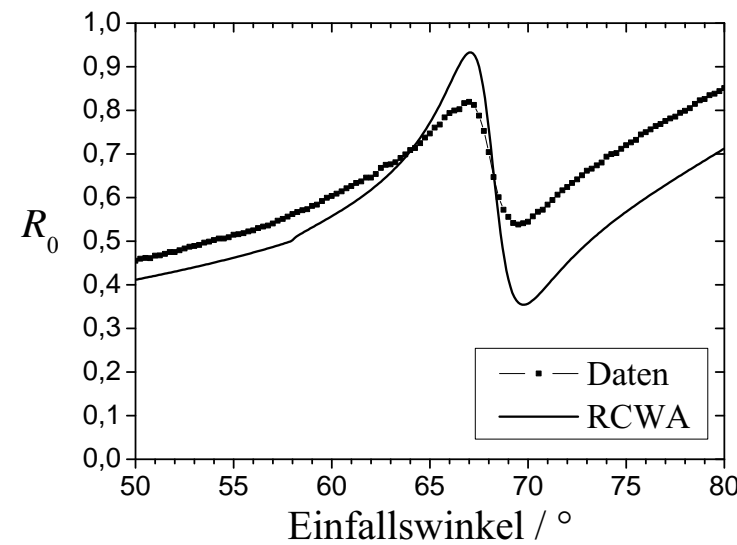

(a) Gemessenes (Daten) und berechnetes (RCWA) Winkelspektrum der Probe DGWS/Hf30/18.

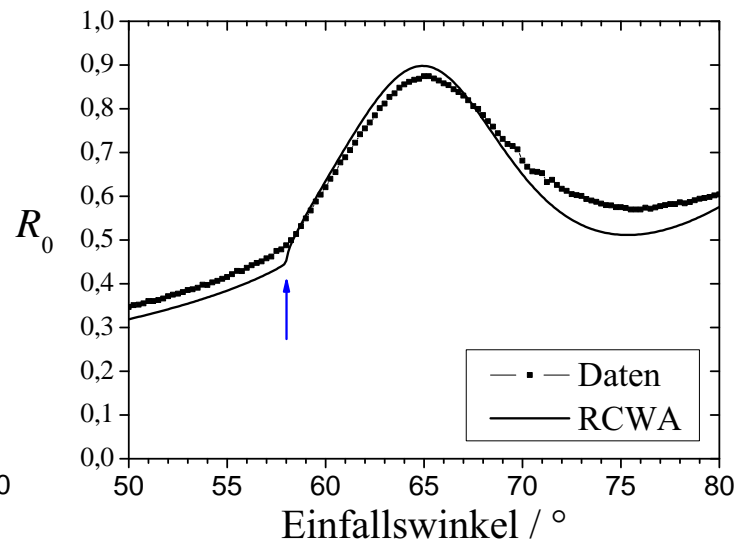

(b) Gemessenes (Daten) und berechnetes (RCWA) Winkelspektrum der Probe DGWS/Hf30/40.

Abb. 4.3: Gemessenes (Daten) und berechnetes (RCWA) Winkelspektrum der Reflexion nullter Ordnung der (a) Probe DGWS/Hf30/18 bzw. (b) DGWS/Hf30/40 für TE-polarisiertes Licht der Wellenlänge $266 \mathrm{~nm}$.

für diese Diskrepanz von bis zu $22^{\circ}$ ist die Brechzahl des Wellenleitermaterials $\mathrm{HfO}_{2} \mathrm{zu}$ sehen (s. u.).

Aus den Resonanzspektren der Proben DGWS/Hf30/40 und DGWS/Hf30/18 in Abb. 4.3 können zudem die Reflektivitäten von bis zu $87 \pm 1 \%$ ausgemacht werden. Bedingt durch das Auftreten höherer Beugungsordnungen (für den gezeigten Winkelbereich bis zu 6 Ordnungen innerhalb der hochbrechenden Wellenleiterschicht), welche durch die im Verhältnis zur Wellenlänge kleine Gitterperiode von $360 \mathrm{~nm}$ verursacht werden, liegen die berechneten Reflektivitäten nicht auf einem $100 \%$-Niveau, sondern erreichen für die Probe DGWS/Hf30/18 etwa $93 \pm 1 \%$ bzw. für die Probe DGWS/Hf30/40 knapp 90 $\pm 1 \%$ (s. u.). Zudem weisen die Messungen aufgrund des großen Einfallswinkels eine hohe FRESNEL-Reflektivität von etwa 30$40 \%$ auf. Insbesondere bei der Probe DGWS/Hf30/18 übersteigen die gemessenen Werte die berechneten; wobei sich dieser Effekt mit zunehmendem Einfallswinkel verstärkt. Hinzu kommt, dass bei dieser Probe die gemessene Reflektivität von $82 \pm 1 \%$ rund $12 \%$ unter dem berechneten Wert liegt. Dies kann damit begründet werden, dass für große Winkel die effektiv bestrahlte Gitterfläche kleiner wird und somit der Strahldurchmesser nach der Strahlreduzierungsoptik nicht mehr in- 
nerhalb der ca. 0,5 mm breiten Gitterstruktur liegt, d. h. dass auch Bereiche ohne Gitter durchstrahlt werden und damit nicht zur Resonanz beitragen. So liegt die belichtete Gitterbreite bei der Bestrahlung der Probe im Resonanzwinkel $\theta_{\mathrm{R}}$ bereits bei $\cos \theta_{\mathrm{R}} \approx 0,2 \mathrm{~mm}$, was einem Intensitätsverlust (bezogen auf $1 / \mathrm{e}^{2}$-Abfall) von ca. $50 \%$ entspricht. Eine weitere Reduzierung des Strahldurchmessers war aufgrund der ungenügenden Strahlqualität jedoch nicht möglich (Beugungsbegrenzung).

Die in Abb. 4.3b sichtbare und durch einen blauen Pfeil gekennzeichnete WooDsche Anomalie bei $58^{\circ}$ Einfall macht deutlich, dass die Resonanz bereits nahe der Abbruchkante für die Totalreflexion der +1 . Beugungsordnung (Wellenleiterbedingung) stattfindet. Aufgrund dieser Kante ist auch die Anregung der TM-Resonanz, die in der Nähe dieses Winkels zu erwarten wäre, nicht möglich. Über die analytische Beschreibung der WooDschen Anomalie $n_{2}=\lambda_{0} / \Lambda+\sin \theta_{0}$ [vgl. (2.43)] kann die Brechzahl für das Substrat D 263 T, welche für $\lambda_{0}=266 \mathrm{~nm}$ nicht bekannt ist, berechnet werden. Diese beträgt für die Probe DGWS/Hf30/40 bei gegebener Gitterperiode $\Lambda=360 \mathrm{~nm}$ für diesen Winkel $\theta_{0}$ etwa 1,58(7).

Für die Berechnung des Resonanzverhaltens in Reflexion mithilfe der RCWA. Methode wird bei beiden Proben der mit dieser Methode bestimmte Brechungsindex des Substrats benutzt. Die Brechzahl der wellenleitenden Schichten wird (ohne Kenntnis über deren tatsächlichen Wert) hingegen so angepasst, dass die berechneten Kurven möglichst dem gemessenen Resonanzverhalten entsprechen. So wird ein Brechungsindex von 2,267 für die Probe DGWS/Hf30/40 bzw. 2,244 für die Probe DGWS/Hf30/18 als Berechnungsgrundlage angenommen. Mithilfe dieser Anpassung wird aus der Differenz der Reflektivitäten aus Theorie und Experiment der Streu- bzw. Absorptionsanteil der Proben in der Summe $(S+A)$ auf ca. $3 \%$ geschätzt. Wie bereits in Abschnitt 3.5 angedeutet, ist davon auszugehen, dass bei der Beschichtung der Probe DGWS/Hf30/40 mit einer Schichtdicke von $30 \mathrm{~nm}$ keine über die gesamte Gitterstruktur durchgehende Wellenleiterschicht ausgebildet wurde. Da die RCWA-Simulationen, deren Berechnungen auf genau diesem Strukturmodell basieren, mit den experimentellen Daten der Probe vergleichsweise gut übereinstimmen, scheint diese Annahme berechtigt zu sein.

Abb. 4.4 zeigt die Ergebnisse der Transmissions- und Reflexionsmessungen bei resonanter Bestrahlung der Probe DGWS/Hf74/40 mit Laserlicht der Wellenlänge 


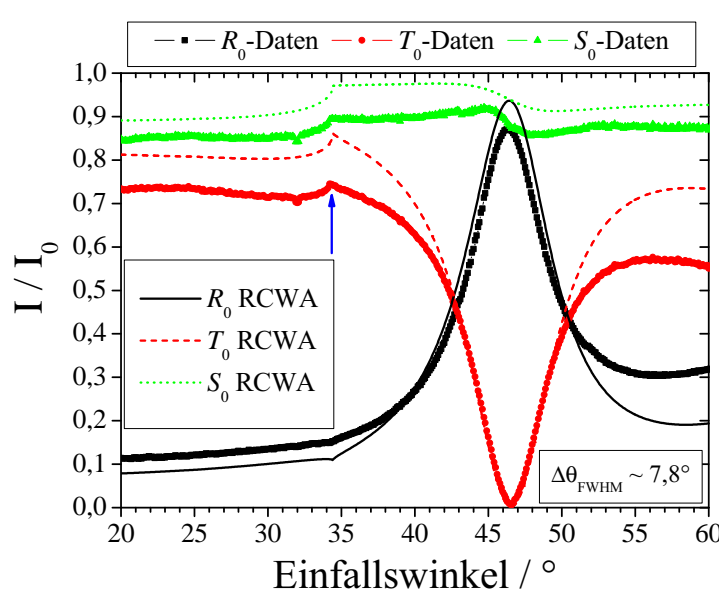

(a) Reflexion und Transmission der Probe DGWS/Hf74/40.

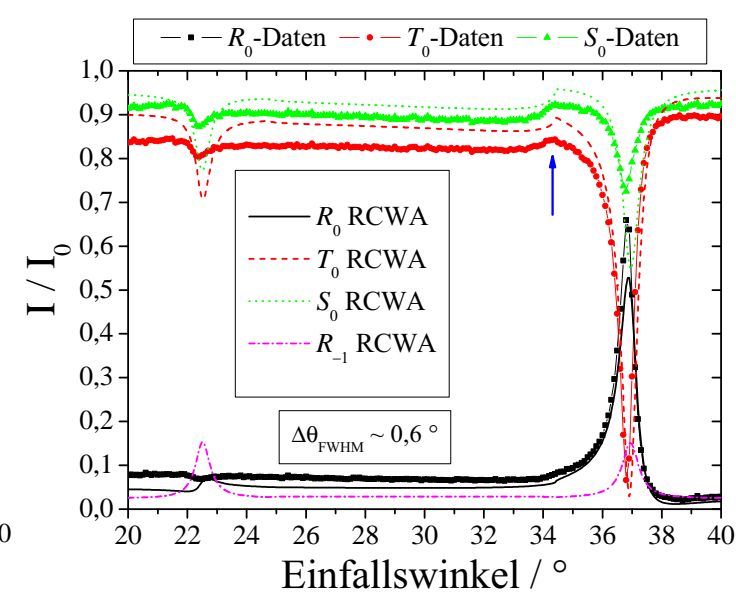

(b) Reflexion und Transmission der Probe DGWS/Hf74/40.

Abb. 4.4: Aufgenommene (Daten) und berechnete (RCWA) Intensitätsverteilung über dem Winkelspektrum bei resonanter Anregung der Probe DGWS/Hf74/40 mit (a) TE-polarisiertem bzw. (b) TM-polarisiertem UV-Licht der Wellenlänge $355 \mathrm{~nm}$. Dargestellt sind die jeweils 0 . Beugungsordnung der Reflexion $\left(R_{0}\right)$, der Transmission ( $\left.T_{0}\right)$ und der Summe aus $R_{0}+T_{0}\left(S_{0}\right)$.

354,7 nm. Abb. 4.4a stellt dabei die Verteilung der normierten Intensitäten über einen Winkelbereich von 20-60 bei TE-polarisiertem UV-Licht dar; wohingegen Abb. 4.4b die TM-Anregung der Probe über einen nur halb so großen Bereich von 20-40 abbildet. Wie Abb.4.4a außerdem zeigt, liegt der Resonanzwinkel bei Anregung der Probe mit TE polarisiertem Laserlicht bei 46, $5^{\circ}$. Das Reflexionsmaximum beträgt demnach $87 \pm 1 \%$, während die Intensität in Transmission nahezu vollständig einbricht $(<1 \%)$. Die deutlich schmalere TM-Resonanz mit einer maximalen Reflektivität von $67 \pm 1 \%$ und einem Intensitätsminimum in Transmission von etwa $7 \pm 1 \%$ besitzt ihr Maximum (bzw. Minimum) bei einem Resonanzwinkel von etwa $36,8^{\circ}$. Damit sind die angestrebten Resonanzwinkel von $+45^{\circ}$ für TE und $+30^{\circ}$ für TM-Anregung nahezu erreicht worden. Sämtliche gemessene Intensitäten werden wie zuvor bei den Proben DGWS/Hf30/18 und DGWS/Hf30/40 aufgrund des ungünstigen Verhältnisses von Gitterperiode und Wellenlänge durch die Anregung höherer Beugungsordnungen (bis zu 4) beeinflusst.

Darüber hinaus sind in Abb. 4.4 auch die berechneten Resonanzkurven (RCWA dargestellt, deren Reflektivitäten bei $94 \%$ für TE bzw. $54 \%$ für TM-polarisiertes 
Licht liegen. Wie zuvor in Abb. 4.3 wird das berechnete dem Resonanzverhalten gemessenen über den Brechungsindex des Wellenleiters angeglichen: Die bestmögliche Anpassung der Maxima bzw. Minima gelingt bei den Wellenleiterbrechzahlen 2,113 (TE) und 2,082 (TM). Darüber hinaus kann über die in Abb. 4.4 gezeigte Woodsche Anomalie (blaue Pfeile) der Brechungsindex des Substrats bestimmt werden. Für diesen spezifischen Winkel von 34,3 beträgt die Brechzahl 1,54(9) und ist damit nahezu identisch mit dem vom Hersteller Schotт $^{\circledR}$ für das Substrat D 263 T angegebenen Brechungsindex von 1,54875, welcher mithilfe der i-Linie $(365,0146 \mathrm{~nm})$ einer Quecksilberdampflampe bestimmt worden ist.

Weiterhin ist ein deutlicher Unterschied in der Resonanzwinkel-(Halbwerts-)Breite $\Delta \theta_{\text {FWHM }}$ beider Anregungen zu erkennen, die bei TE-Anregung in Reflexion ca. $7,8^{\circ}$ breit ist und bei der Anregung mit TM-polarisiertem Licht eine Breite von rund $0,6^{\circ}$ besitzt. Damit ist $\Delta \theta_{\text {FWHM }}$ bei der TE-Resonanz über eine Größenordnung breiter als das Resonanzspektrum bei TM-Anregung.

Beim Vergleich der gemessenen und berechneten Summen $S_{0}$ (grün dargestellt) wird deutlich, dass für den kompletten Winkelbereich ein mittlerer Differenzbetrag zwischen beiden Summen von ungefähr $6 \pm 1 \%$ (TE) bzw. rund $3 \pm 1 \%$ (TM) besteht. Damit kann die unter Abschnitt 4.2 angesprochene und für $m=0$ angepasste Intensitätsgleichung $R_{0}+T_{0}+(A+S)<1$ zur Abschätzung von $(A+S)$ herangezogen werden. Danach kann ein Mittelwert von 4,5 $\pm 1 \%$ der mittleren Differenzbeträge der Summe aus Absorption $A$ und Streuung $S$ zugeordnet werden.

Zudem zeigt Abb. 4.4b beim Resonanzwinkel von 36,8 einen deutlichen Intensitätseinbruch in der Summendarstellung $S_{0}$. Wie man vielleicht zunächst vermuten mag, geht dieser jedoch nicht auf einen Absorptionsprozess während der Kopplung zurück - einen ähnlichen Einbruch hätte man auch in Abb. 4.4a sehen müssen -, sondern wird auf eine zusätzliche resonante Anregung der Probe zurückgeführt. Die in Abb. 4.4b dargestellte, berechnete Reflexion -1. Beugungsordnung $R_{-1}$ macht deutlich, dass es sich hierbei um eine Überlagerung von Resonanzen handelt. Wie man jedoch anhand der Reflektivitäten erkennt, kommt dieser Effekt im Experiment weniger zum Tragen als bei der numerischen Berechnung mittels RCWA. Darüber hinaus ist das Resonanzphänomen bei einem Einfallswinkel von $22,5^{\circ} \mathrm{zu}$ sehen, welches die Resonanz bei $36,8^{\circ}$ zwar nicht unmittelbar beeinflusst, aber dennoch im Zusammenhang mit ihr steht. 
Zum besseren Verständnis sind in Abb. 4.5 die Resonanzkennlinien TEb und TM, der Probe DGWS/Hf74/40 unter Berücksichtigung der 1. und 2. Beugungsordnung dargestellt. Zusätzlich ist eine magentafarbene Linie eingezeichnet, welche die Kennlinien bei der Wellenlänge $355 \mathrm{~nm}$ schneidet. Die Schnittpunkte auf der TM-Kennlinie (Magentakreise) liegen für die mittels +1 . Beugungsordnung angeregte Mode bei ca. $35^{\circ}$ bzw. bei $-24,3^{\circ}$ für die Kennlinie -2 . Beugungsordnung. Beide Winkel können den in Abb. 4.4b gezeigten Resonanzwinkeln $36,8^{\circ}$ und $22,5^{\circ}$ zugeordnet werden. Damit wird deutlich, dass beide Resonanzen aufgrund der unterschiedlichen Resonanzwinkel verschiedene Ereignisse markieren, sich dennoch bei der Anregung höherer Beugungsordnungen - aufgrund der Phasenbeziehung aller gebeugten Teilstrahlen - gegenseitig beeinflussen. Wie Abb. 4.5 verdeutlicht, betrifft das Phänomen der Resonanzüberlagerung auch die $\mathrm{TE}_{\mathrm{b}}$ Kennlinie. Diese ist jedoch in Reflexion

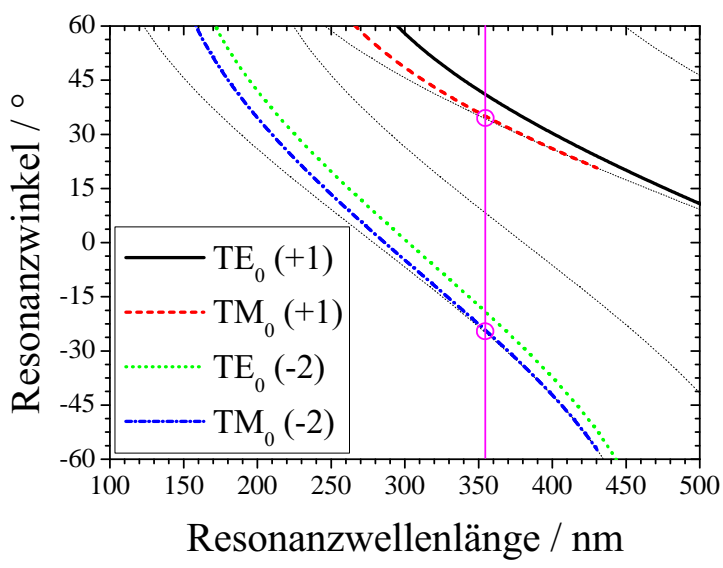

Abb. 4.5: Berechnete Resonanzkennlinien der Probe DGWS/Hf74/40 unter Berücksichtigung +1 . und -2 . Beugungsordnung. (sowohl experimentell als auch theoretisch) nicht so stark ausgeprägt.

\subsubsection{Spektrale Bestimmung der Resonanz mit breitbandigem Laserlicht}

Mithilfe eines Titan:Saphir-Lasers, welcher die Eigenschaft besitzt, ein relativ breites Emissionsspektrum zu erzeugen, ist es möglich, die spektrale Resonanz von DWG mit diesem Laserspektrum vollständig abzudecken und damit zu charakterisieren. Bei dieser Art von Festkörperlaser, welcher zu den vibronischen Lasern gehört, wird als aktives Medium ein mit Titan (Ti) dotierter SaphirKristall ( $\mathrm{Ti}: \mathrm{Al}_{2} \mathrm{O}_{3}$ ) verwendet. Aufgrund der spezifischen Kopplung der elektronischen Zustände des $\mathrm{Ti}^{3+}$-Kations mit den Phononen des Wirtskristalls zeigt der Ti:Saphir-Kristall eine vergleichsweise breite Fluoreszenzbande von 670-1070 nm 
mit einem Maximum bei $800 \mathrm{~nm}$. Diese Eigenschaft ermöglicht es, mit einer monochromatischen Pumpfrequenz ein breitbandiges Emissionsspektrum anzuregen und mithilfe eines dispersiven Elements den kompletten Fluoreszenzbereich durchzustimmen (MOUlTON 1982 ; EICHLER und EICHLER 2006$)$.

Zur Charakterisierung der Proben DGWS/Ta150/24, DGWS/Ta150/40 und GWS/Nb150/42 wird ein modengekoppelter Ti:Saphir-Laser vom Typ Tsunami (Spectra Physics) verwendet, der über einen $\mathrm{Nd}$ : $\mathrm{YVO}_{4}$-Festkörperlaser (MillenniaX, SpeCtra Physics) der frequenzverdoppelten Wellenlänge $532 \mathrm{~nm}$ mit $10 \mathrm{~W}$ Ausgangsleistung gepumpt wird. Der angeregte Ti:Saphir-Laser emittiert bandbreitenbegrenzte Laserpulse mit einer Pulsdauer von ca. $100 \mathrm{fs}$ bei einer Repetitionsrate von $82 \mathrm{MHz}$ (quasi-CW). Zur Analyse des Emissionsspektrums wird der Detektorkopf eines Spektrographen vom Typ RE201 (Imaging \& SEnsing Technology) verwendet, welcher die Darstellung des Spektrums über ein Oszilloskop (bzw. Oszillograph) ermöglicht. Zur spektralen Vermessung der Proben wird der Ti:Saphir-Laser auf eine zentrale Wellenlänge von $800 \mathrm{~nm}$ abgestimmt. Mithilfe des Oszilloskops wird die spektrale Pulsbreite der emittierten Laserpulse auf etwa $10 \mathrm{~nm}$ bestimmt.

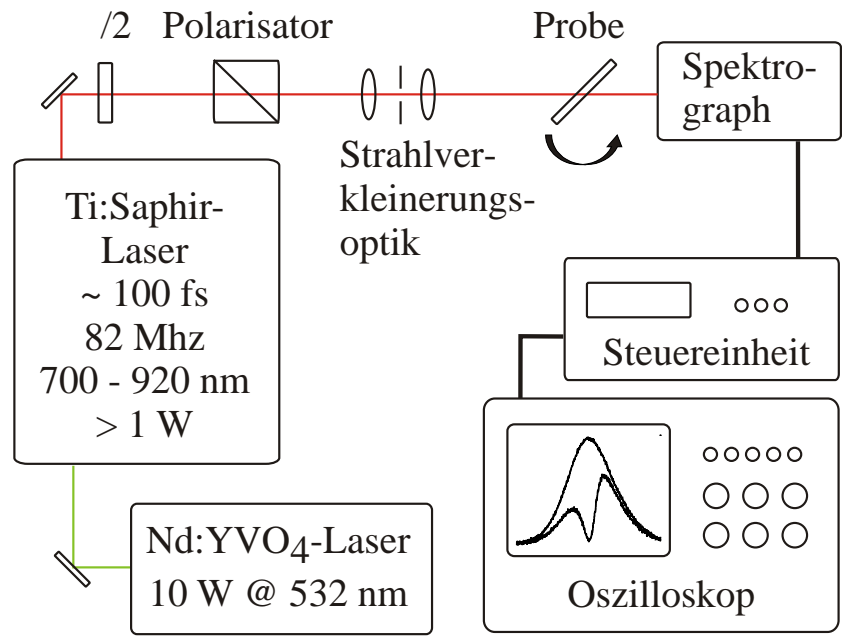

Abb. 4.6: Messanordnung mit Strahlengang des Titan:Saphir-Lasers zur spektralen Vermessung der Proben DGWS/Ta150/40 und GWS/Nb150/42 sowie der Probe GWS/Nb150/42.
Abb. 4.6 zeigt die zur spektralen Charakterisierung der DWG. Proben verwendete Messanordnung in einer schematischen Darstellung. Durch die Kombination einer $\lambda / 2$-Platte und einem Polarisator, welcher die Einstellung der Laserstrahlpolarisation zulässt, kann die Energie des einfallenden Laserlichts verändert werden. Die Ausgangsenergie des Lasers wird mithilfe einer Laser-Steuereinheit und einem mobilen Messkopf (nicht gezeigt) eingestellt und kontrolliert. Um die nur $200 \times 200 \mathrm{\mu m}^{2}$ 


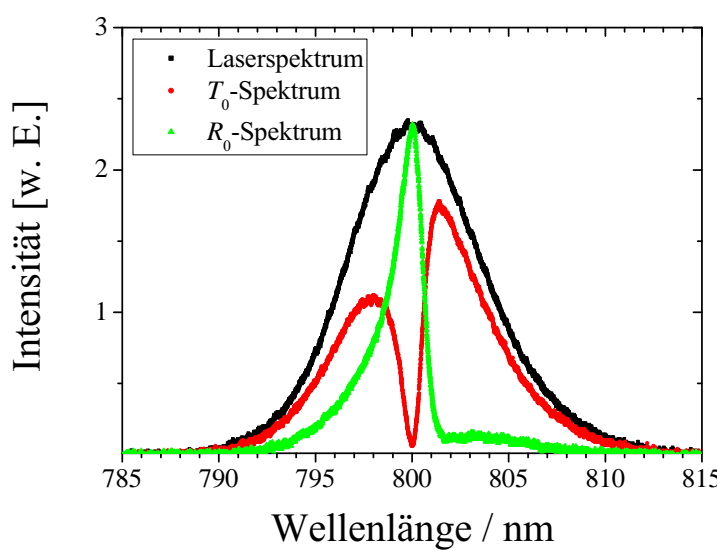

(a) Lineare Darstellung der Spektren.

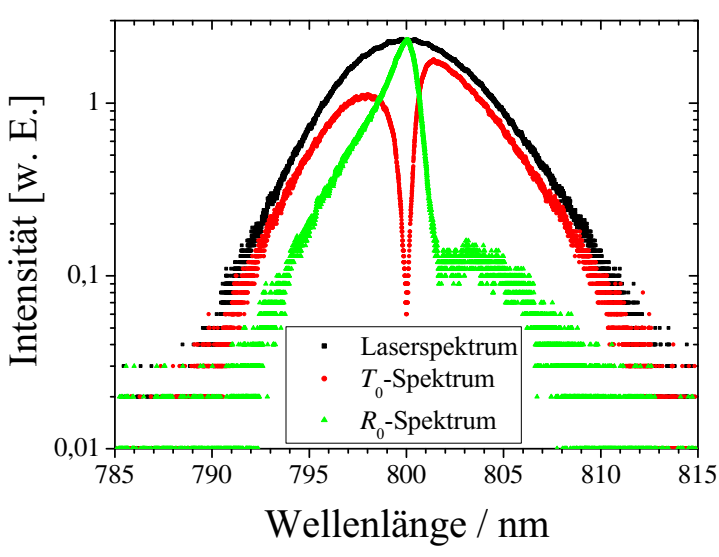

(b) Logarithmische Darstellung der Spektren.

Abb. 4.7: Mittels Oszilloskop aufgezeichnetes Laser- bzw. $T_{0}$ - und $R_{0}$-Spektrum der Probe DGWS/Ta150/24 in (a) linearer und (b) logarithmischer Darstellung.

große Gitterfläche der Probe GWS/Nb150/42 ohne Strahlüberlapp ausleuchten zu können, wird der Laserstrahl räumlich begrenzt. Dazu wird eine Strahlverkleinerungsoptik eingesetzt, welche aus einem geeigneten Linsensystem und einer Lochblende besteht. Die Probe selbst wird auf einem Drehtisch mittels Kevlarschrauben in einem Halter montiert. Dabei ist die Probe stets so ausgerichtet, dass der Gittervektor senkrecht zur Drehachse des Drehtisches steht. Zur Ausrichtung des Einfallswinkels dient die Nonius-Skala des Drehtisches, deren Schrittweite minimal 1/10 Grad beträgt. Bei der Vermessung der Proben wird der Einfallswinkel so eingestellt, dass die Zentralwellenlänge von $800 \mathrm{~nm}$ der Resonanzwellenlänge entspricht. Zur Aufnahme des Transmissionssignals $T_{0}$ befindet sich der Detektorkopf des Spektrographen direkt hinter dem Drehtisch. Um die Proben in Reflexion $\left(R_{0}\right)$ $\mathrm{zu}$ vermessen, wird der Detektor entsprechend dem reflektierten Strahl verschoben. Um das Reflexionssignal anschließend normieren zu können, wird das Reflexionsspektrum eines Aluminiumspiegels, dessen Reflektivität bei der Anregungswellenlänge bekannt ist, aufgenommen. Die mittels Spektrographen aufgezeichneten Messdaten (Spektren) werden auf einem Oszilloskop zunächst dargestellt und anschließend gespeichert.

Abb. 4.7 zeigt das mittels Oszilloskop aufgezeichnete Laserspektrum (ohne Probe) in (a) linearer und (b) logarithmischer Darstellung bei der Zentralwellenlänge 
$800 \mathrm{~nm}$. Zum Vergleich ist in beiden Abbildungen das $T_{0^{-}}$und $R_{0^{-}}$Spektrum bei resonanter Bestrahlung der Probe DGWS/Ta150/24 mit TE-polarisiertem Licht dargestellt. Das innerhalb des Wellenlängenbereichs 785-815 nm linear dargestellte Laserspektrum zeigt eine typische Wellenlängenverteilung nach GAUss. Die logarithmische Darstellung in Abb. 4.7b verdeutlicht hingegen technische Grenzen von Spektro- bzw. Oszillograph: Ab einem intern verarbeiteten Intensitätssignal kleiner 0,1 willkürlicher Einheit w. E. ist deutlich die Diskretisierung des Signals von 0,01 w. E. zu sehen. Da das Signal an den Ausläufern der Spektren schnell abfallend ist, wird eine Auswertung der Messdaten für diesen Bereich erschwert. Die spektrale Aufösung des Spektrographen liegt hingegen bei 3 pm.

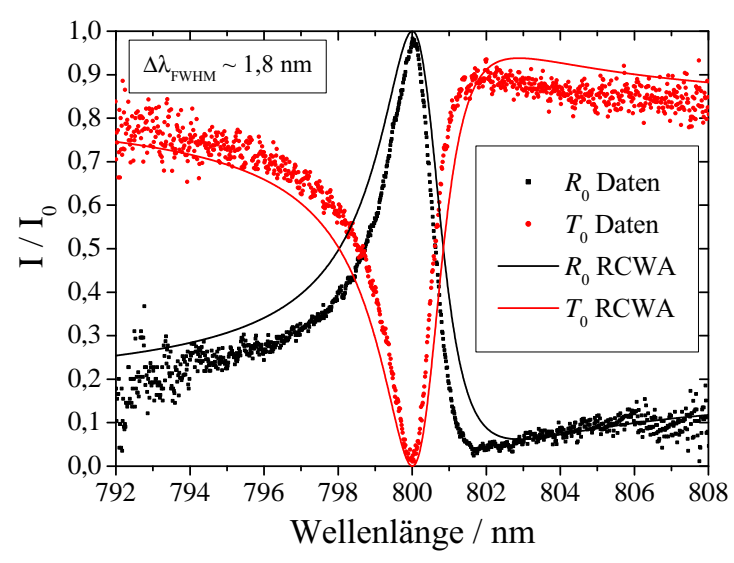

Abb. 4.8: $T_{0^{-}}$und $R_{0^{-}}$Spektrum der Probe DGWS/Ta150/24 für TE polarisiertes Laserlicht.
In Abb. 4.8 sind die ausgewerteten und auf das Laserspektrum ohne Probe normierten Intensitäten der Probe DGWS/Ta150/24 dargestellt. Der mittels Drehtisch eingestellte Resonanzwinkel beträgt bei der Anregung mit TE-polarisiertem Licht rund $-31,5^{\circ}$. Der Fehler bei der Bestimmung des Einfallswinkels wird auf unter $0,1^{\circ}$ geschätzt. Die spektrale Resonanzbreite $\Delta \lambda_{\overline{\text { FWHM }}}$ um die Zentralwellenlänge $800 \mathrm{~nm}$ zeigt einen nichtsymmetrischen Verlauf, wie er bei diesen relativ großen Einfallswinkeln typisch ist (s. Abschnitt 2.5). Die spektrale Resonanzbreite in Reflexion und Transmission ist im Rahmen der Messgenauigkeit identisch und beträgt etwa 1,8 nm. Die Reflektivität und Transmittivität der Probe wird auf etwa $98 \%$ bzw. $1 \%$ bestimmt. Der Fehler in den Intensitäten der ausgewerteten Spektren beträgt dabei $\pm 2 \%$. Da die $R_{0}$ und $T_{0}$-Spektren nicht zeitgleich erfasst werden, korrelieren sie vergleichsweise schlecht. Zudem wird das Messsignal für Wellenlängen mit zunehmendem Abstand zur Zentralwellenlänge aufgrund des Laserspektrums (s. o.) von starkem Rauschen beeinflusst. Auf die Darstellung der Summe $S_{0}$ aus dem $T_{0^{-}}$und $R_{0}$-Spektrum wird daher verzichtet. 


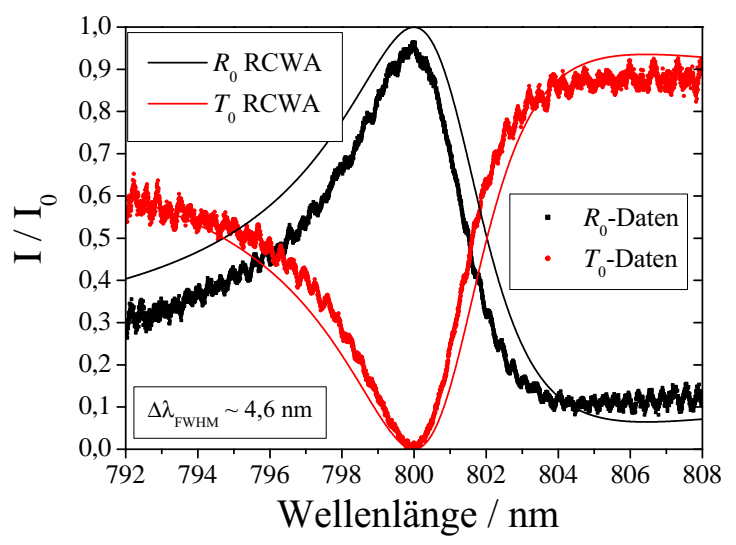

(a) Resonanzspektren der Probe DGWS/Ta150/40 bei TE-Anregung.

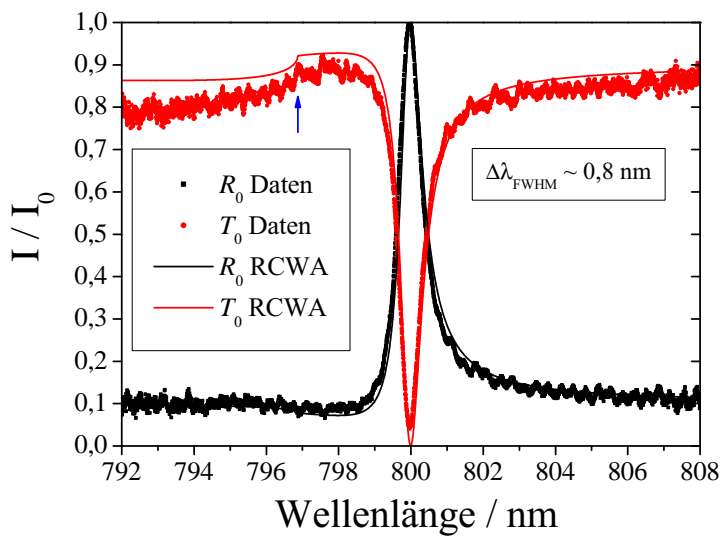

(b) Resonanzspektren der Probe DGWS/Ta150/40 bei TM-Anregung.

Abb. 4.9: Reflexions- und Transmissionsspektrum der Probe DGWS/Ta150/40 für (a) TE bzw. (b) TM-polarisiertes Laserlicht.

Zusätzlich zu den Messdaten sind in Abb. 4.8 auch die mittels RCWA berechneten Resonanzspektren der Probe DGWS/Ta150/24 dargestellt. Durch die Bestimmung des TM-Resonanzwinkels bei Anregung der Probe mit TM-polarisiertem Licht auf $-42,8 \pm 0,1^{\circ}$ (nicht gezeigt) ist es möglich, die relativ ungenauen Strukturdaten des Wellenleiters bei der Berechnung so anzupassen, dass die Resonanzwinkel der jeweiligen Spektren übereinstimmen. Mit einer Wellenleiterdicke von 124,3 nm und einem Brechungsindex von 2,092 gelingt die Anpassung der Spektren am besten. Die dadurch angenommene effektive Wellenleiterdicke beträgt somit 148,3 nm und entspricht damit nahezu der vom Hersteller angegebenen Schichtdicke von $150 \mathrm{~nm}$. Die zur Berechnung verwendete Brechzahl des Substrats AF 45 beträgt für die Zentralwellenlänge 1,5202.

Abb. 4.9 zeigt die ausgewerteten und auf den Laserpuls ohne Probe normierten Resonanzspektren der Probe DGWS/Ta150/40 in $T_{0}$ und $R_{0}$ für TE und TM. polarisiertes Licht $4.9 \mathrm{a}$ bzw. 4.9b). Der mittels Drehtisch eingestellte Resonanzwinkel beträgt bei TE-Resonanz rund $-33,8 \pm 0,1^{\circ}$ und liegt bei Anregung der Probe mit TM-polarisiertem Licht um gut $10^{\circ}$ verschoben bei etwa $-44,1 \pm 0,1^{\circ}$. Die Reflektivität bei der Resonanzwellenlänge $800 \mathrm{~nm}$ wird auf $96 \pm 2 \%$ (TE) bzw. $100 \pm 2 \%$ (TM) bestimmt. Entsprechend liegt die Transmittivität der Probe bei 
$1 \pm 2 \%$ (TE) bzw. $3 \pm 2 \%(\mathrm{TM})$. Die Resonanzbandbreite (FWHM der TE Resonanz in Reflexion stimmt mit der in Transmission ermittelten Bandbreiten $\Delta \lambda_{\text {FWHM }}$ von etwa $4,6 \mathrm{~nm}$ überein. Die spektrale Bandbreite der TM-Resonanz ist mit $0,8 \mathrm{~nm}$ deutlich kleiner.

Die ebenfalls in Abb. 4.9 gezeigten berechneten Spektren (RCWA werden wie zuvor bei der Probe DGWS/Ta150/24 an die gemessenen Daten angepasst. Bei gleicher Wellenleiterbrechzahl 2,092 findet eine Deckung der polarisationsabhängigen Resonanzwinkel bei einer Wellenleiterdicke von 94,8 nm statt. Mit einer effektiven Schichtdicke von 134,8 nm wird jedoch eine Diskrepanz zu dem vom Hersteller angegebenen Wert von $150 \mathrm{~nm}$ deutlich. Nach Rücksprache mit dem Hersteller räumt dieser jedoch ein, dass durchaus eine falsche Spezifizierung der als Testmuster anzusehenden Proben denkbar ist und schließt deshalb nicht aus, dass die tatsächlich gefertigte Wellenleiterschicht u. U. eine Dicke von $130 \mathrm{~nm}$ besitzt - welche ebenfalls vom Hersteller angeboten wird. Da sich die nachträgliche Untersuchung der Schicht jedoch als schwierig erweist, kann die Abweichung der Schichtdicke nicht abschließend geklärt werden. Bei der Berechnung der Spektren wird darüber hinaus als Substratbrechungsindex der Wert 1,5176 verwendet, welcher mit dem über die in Abb. $4.9 \mathrm{~b}$ sichtbare Woodsche Anomalie (blauer Pfeil) berechneten Wert von rund 1,51(7) im Rahmen der Messgenauigkeit identisch ist.

Beim Vergleich der TE-Resonanzspektren der DGWStProben DGWS/Ta150/24 und DGWS/Ta150/40 ist auffällig, dass die gezeigten FWHM-Bandbreiten unterschiedlich groß sind. Dieses Verhalten ist auf die unterschiedliche Gittertiefe der Proben zurückzuführen und lässt sich (näherungsweise) analytisch mit dem quadratischen Anstieg der Resonanzbandbreite mit zunehmender Gittertiefe beschreiben (s. Abschnitt 2.4.3). Der Theorie nach beträgt der Faktor zwischen den Tiefen etwa 2,8, welcher dem experimentell bestimmten Wert von rund 2,6 sehr nahe kommt. Die reflektierten Pulslängen der Proben DGWS/Ta150/24 und DGWS/Ta150/40 unter Berücksichtigung des FrESNEL-Anteils werden in Abschnitt 4.3 untersucht.

In Abb. 4.10 ist das spektrale Resonanzverhalten der Probe GWS/Nb150/42 in $T_{0}$ und $R_{0}$ bei der Zentral- bzw. Resonanzwellenlänge $800 \mathrm{~nm}$ dargestellt. Da die mittels $\mathrm{F}_{2}$-Laserablation erzeugte Gitterstruktur vergleichsweise inhomogen ist (s. Abb. 3.5), wird eine geeignete Stelle auf der Probe mit maximierter Anregung 
durch TE-polarisiertes Laserlicht erst nach mehreren Anläufen gefunden. Der ermittelte Resonanzwinkel beträgt an dieser Stelle bei einer maximalen Reflektivität von $44 \%$ rund $42 \pm 0,1^{\circ}$. Das Transmissionsminimum wird auf ca. $25 \%$ bestimmt; wobei der Messfehler der Intensität in den gezeigten Spektren wie zuvor auf $\pm 2 \%$ geschätzt wird. Die spektrale Resonanzbreite $\Delta \lambda \overline{\text { FWHM }}$ beträgt ungefähr $6,5 \mathrm{~nm}$.

Zusätzlich zu den experimentellen Daten sind auch die berechneten bzw. angepassten Spektren (RCWA dargestellt. Zusätzlich zu den in Abb. 3.1 gezeigten Strukturdaten geht auch die im Vorfeld der Strukturierung bestimmte komplexe Brechzahl des Wellenleiters in die RCWA-Berechnungen mit ein. Der gemessene Extinktionskoeffizient $\kappa$ der unstrukturierten Probenschicht beträgt für die Wellenlänge von $800 \mathrm{~nm}$ etwa $6,8 \cdot 10^{-3}$; der Brechungsindex hingegen geht mit einem Wert von 2,135(5) in die Simulation ein. Um

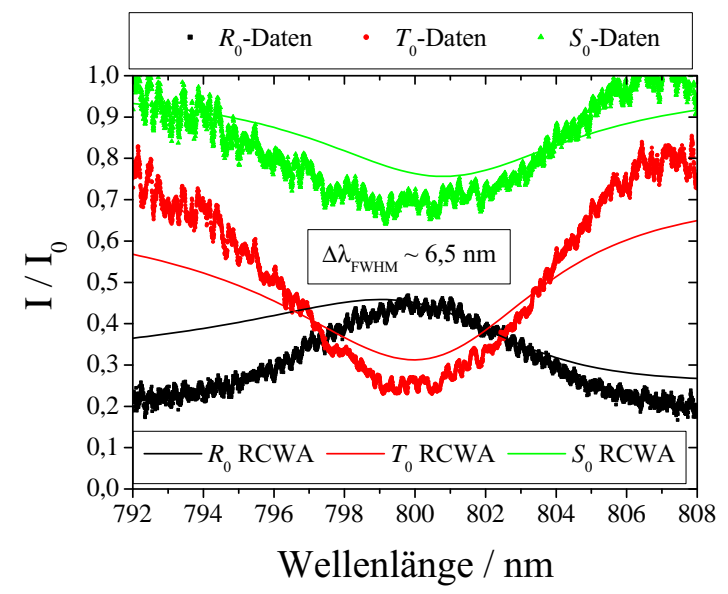

Abb. 4.10: $T_{0}$ - und $R_{0}$-Spektrum der Probe GWS/Nb150/42 für TE-polarisiertes Laserlicht. die berechneten Spektren mit den gemessenen bei der Resonanzwellenlänge $800 \mathrm{~nm}$ zur Deckung zu bringen, wird eine Wellenleiterdicke von 117,7 nm (effektive Dicke 138,7 nm mit Gittertiefe $42 \mathrm{~nm}$ und Füllfaktor 0,5) als Berechnungsgrundlage verwendet. Als Brechzahl für das Quarzglassubstrat wird ein Wert von 1,46 angenommen.

Der vergleichsweise hohe Einbruch im dargestellten Summenspektrum $S_{0}$ bei der Resonanzwellenlänge macht deutlich, dass hier durch die wellenleitende Schicht eine Materialabsorption stattfindet und - wie schon erwähnt - bereits bestimmt worden ist. Anhand des schnellen Anstiegs der Summenintensität außerhalb der Resonanz können Streuverluste an dieser Stelle der Probe weitgehend ausgeschlossen werden. Allein dieser Absorptionsprozess stört die unter Resonanzbedingungen auftretende Interferenz so nachhaltig, dass das Reflexionsmaximum und das Transmissionsminimum entsprechend beeinflusst werden. Begleitet wird dieser Prozess von einem Wellenlängenversatz zwischen dem Reflexionsmaximum und dem Trans- 
missionsminimum, welcher jedoch innerhalb der gemessenen Spektren augenscheinlich nicht zum Tragen kommt.

\subsubsection{Spektrale Messungen mit monochromatischem Licht}

Zur Charakterisierung der DGWS-Proben DGWS/Hf331/18, DGWS/Nb487/40 und DGWS/Nb551/40 wird ein durchstimmbarer Farbstofflaser verwendet. Durch den Einsatz spezieller Fluoreszenzfarbstoffe als optisch aktives Medium innerhalb des Laserresonators kann je nach verwendetem Farbstoff ein spektraler Bereich von typischerweise 30-60 nm abgedeckt werden - unter Berücksichtigung aller für den Laser zugelassenen Farbstoffe sogar ein Bereich von 320-860 nm. Die Selektion der Wellenlänge des jeweiligen Spektrums geschieht über einen spezifischen Monochromator.

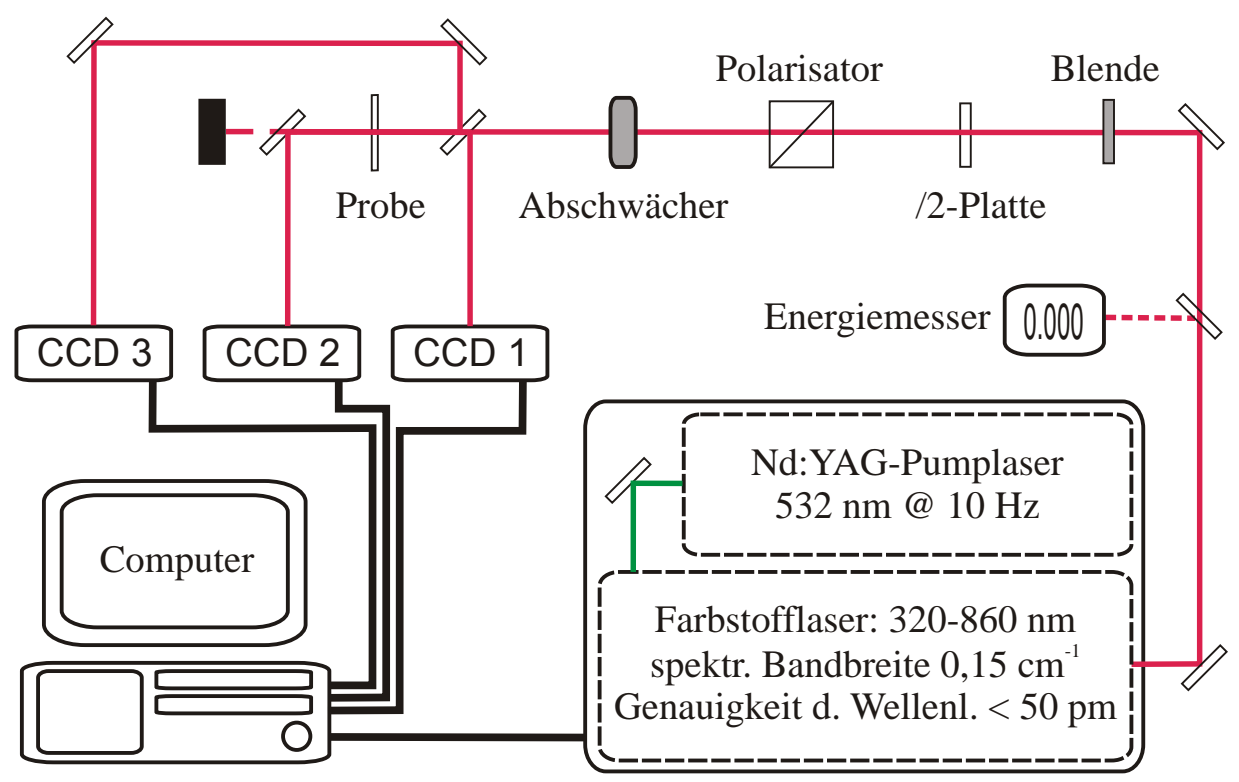

Abb. 4.11: Messanordnung mit Strahlengang des Farbstofflasers zur spektralen Vermessung der Proben DGWS/Hf331/18, DGWS/Nb487/40 und DGWS/Nb551/40.

Zur Vermessung dieser Proben werden zwei Farbstoffe verwendet: Der Farbstoff Pyridin 1, der einen Spektralbereich von 665-725 nm abdeckt, wird zur Charakterisierung der Proben DGWS/Nb487/40 und DGWS/Nb551/40 eingesetzt. Die Zielwellenlänge der beiden Proben ist die des Rubinlasers bei $694,3 \mathrm{~nm}$ ( $R_{1}$-Linie). 
Der Farbstoff DCM, dessen Emissionsspektrum im Wellenlängenbereich von 607$663 \mathrm{~nm}$ liegt, wird hingegen für die Bestrahlung der Probe DGWS/Hf331/18 nahe der Helium-Neon-Laserwellenlänge $632,816 \mathrm{~nm}\left(3 \mathrm{~s}_{2} \rightarrow 2 \mathrm{p}_{4}\right.$ Übergang) benutzt.

Abb. 4.11 zeigt schematisch die Anordnung des Strahlengangs zusammen mit dem verwendeten Farbstofflaser vom Typ ScanMatePro ${ }^{\text {TM }}$ (COHEREnT) und den optischen Komponenten. Zur Anregung der Farbstoffe dient ein frequenzverdoppelter Nd:YAG Pumplaserstrahl der Wellenlänge 532,07 nm, welcher bei einer Repetitionsrate von $10 \mathrm{~Hz}$ ca. $7 \mathrm{~ns}$ lange Laserpulse emittiert. Die Linienbreite des Lasers wird herstellerseitig mit $0,151 / \mathrm{cm}$ angegeben, was einer spektralen Linienbreite (Bandbreite) von rund $6 \mathrm{pm}$ für die Zielwellenlänge $632,816 \mathrm{~nm}$ bzw. 7,2 pm für die Wellenlänge 694,3 nm entspricht. Die Genauigkeit der Emissionswellenlänge des Lasers liegt laut Spezifikation des Herstellers bei unter 50 pm. Der Divergenzwinkel des direkt emittierten Laserstrahls ist herstellerseitig mit 0,5 mrad angegeben.

Mit Hilfe eines Échelle-Spektrometers vom Typ SpectraStar S150HR (SOLAR LASER Systems), welches nach Herstellerangaben eine Genauigkeit von \pm 3 pm besitzt, wird eine spektrale Verschiebung der Laserlinien um ca. 30 pm hin zu längeren Wellenlängen bestimmt. Diese wird bei der späteren Auswertung der Resonanzspektren berücksichtigt. Mit der spektralen Aufösung des Spektrometers von $\lambda / \Delta \lambda_{\text {FWHM }}=3 \cdot 10^{4}$, was für die Wellenlänge 694,3 nm einer Auflösung von $23 \mathrm{pm}$ entspricht, kann die tatsächlich emittierte Linienbreite des Laserstrahls jedoch nur abgeschätzt werden (s. Abschnitt 4.4).

Zur Bestrahlung der Proben wird der Laserstrahl über geeignete Spiegel auf die zu untersuchende Probe gelenkt. Zur gleichzeitigen Erfassung des Referenz-, Transmissions- und Reflexionssignals werden zudem Strahlteiler eingesetzt, die einen keilförmigen Querschnitt besitzen und damit eine Separation der bei vorderund rückseitiger Reflexion auftretenden Teilstrahlen ermöglicht. Der Strahlengang wird dabei so angepasst, dass lediglich der vorderseitige Reflex bei der Detektion berücksichtigt wird. Zudem wird ein weiterer Strahlteiler zur Beobachtung evtl. auftretender Energieschwankungen des Laserstrahls benutzt. Der im Durchmesser ca. $3 \mathrm{~mm}$ große Laserstrahl wird über eine Lochblende im Strahlengang räumlich begrenzt und anschließend über die Kombination von $\lambda / 2$-Platte und Polarisator in seiner Energie und Polarisationsausrichtung (TE bzw. TM voreingestellt. 
Bei allen Messungen wird der Einfalls- bzw. Resonanzwinkel mithilfe eines Goniometers auf $0^{\circ}$ mit einer Ungenauigkeit von $\pm 0,05^{\circ}$ eingestellt. Der Justage- bzw. Ablesefehler des Einfallswinkels wird in der Summe auf unter $\pm 0,1^{\circ}$ geschätzt.

Zur Detektion der von den Proben transmittierten und reflektierten Intensitätssignale werden zeitgleich bis zu drei CCD-Kameras des Typs ML3720 (12-Bit Dynamik) der Firma Metrolux eingesetzt. Alle eingesetzten Kameras sind über die sog. FireWire-Schnittstelle (Standard IEEE1394a) mit einem Computer verbunden und werden über eine spezielle Trigger-Einheit (ML1630) synchron zum Laser angesteuert. Die Ansteuerung der Kameras sowie die Auswertung der einzelnen Kamerabilder geschehen über die Strahlprofilanalyse-Software BEAMLuX II (Version 2.3.4 und 2.4.3). Von den baugleichen Kameras wird die mit der Bezeichnung CCD 1 (s. Abb.4.11), welche insbesondere zur Kompensation der Intensitätsschwankungen des Lasers verwendet wird, zur Detektion eines Referenzsignals eingesetzt. Die Kameras mit den Bezeichnungen CCD 2 und CCD 3 erfassen hinge-

gen das Transmissions- bzw. Reflexionssignal während der Messung. Um die CCD. Kameras bei Pulsenergien von 0,5-1,5 mJ nicht zu beschädigen, werden zusätzlich verschieden starke Graufilter zur Abschwächung der Laserleistung eingesetzt.

Das Intensitätssignal der jeweiligen Kamera wird von der Software durch Integration der CCD-Daten gewonnen. Da es im laufenden Messbetrieb bei der softwareseitigen Erfassung der Daten gelegentlich zum Verlust einzelner Bilder kommt, wird der Intensitätswert erst nach der Mittelung von 250 Kamerabildern berechnet. Die Verlustrate bei der Bilderfassung beträgt durchschnittlich 1 Bild, maximal 2 Bilder, so dass der Fehler der Intensität durch die Mittelwertbildung weniger als $1 \%$ beträgt. Die Normierung des Reflexionssignals erfolgt mithilfe eines Aluminiumspiegels, welcher für die Wellenlänge $694 \mathrm{~nm}$ bei $3^{\circ}$ Einfall eine Reflektivität von 80,1 $\pm 0,6 \%$ besitzt. Mit der Aufnahme eines Dunkelbildes (ohne Laserlicht) für jede Kamera werden zudem alle Messdaten vom Hintergrundlicht befreit.

\subsubsection{Messungen nahe der Rubinlaser-Wellenlänge}

Zur Charakterisierung der Proben DGWS/Nb487/40 und DGWS/Nb551/40 bei der Rubinlaserwellenlänge 694,3 nm werden die Proben nacheinander in den Proben- 


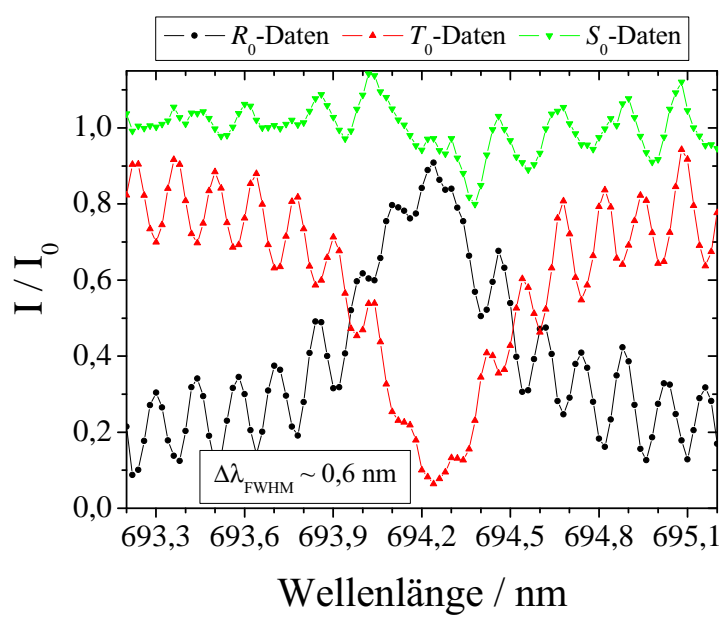

(a) Gemessenes $R_{0^{-}}, \quad T_{0}$ und $S_{0^{-}}$ Spektrum der Probe DGWS/Nb487/40.

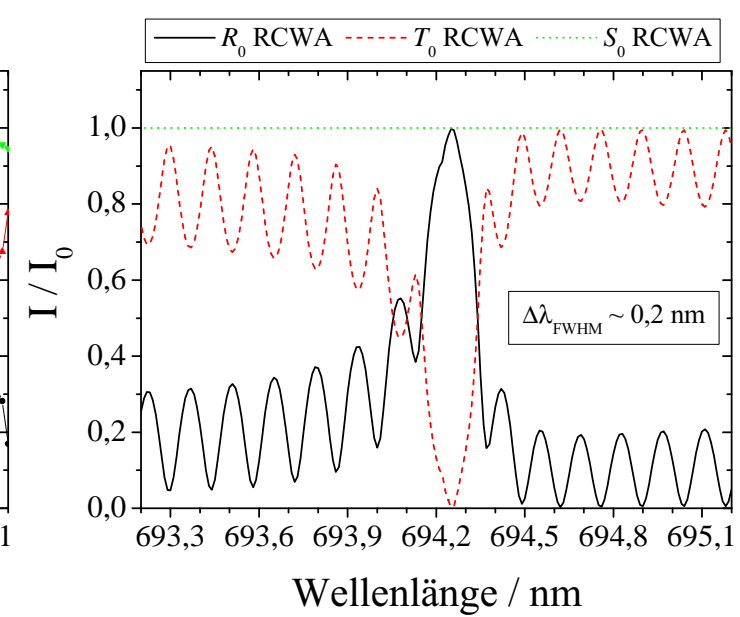

(b) Berechnetes $R_{0^{-}}, \quad T_{0}$ und $S_{0^{-}}$ Spektrum der Probe DGWS/Nb487/40.

Abb. 4.12: Gemessene (a) sowie berechnete (b) $R_{0^{-}}, T_{0}$ und $S_{0^{-}}$Spektren der Probe DGWS/Nb487/40 durch Anregung mit TE/polarisiertem Licht.

halter eingespannt und der Winkel zwischen einfallender Laserstrahlung und Probennormalen auf $0 \pm 0,1^{\circ}$ eingestellt.

In Abb. 4.12a sind die ausgewerteten $R_{0^{-}}$und $T_{0^{-}}$Spektren der Probe DGWS/Nb487/40 für den TE-polarisierten Laserstrahl bei einem Einfallswinkel von $0^{\circ}$ innerhalb des Wellenlängenbereichs von 693,2-695,2 nm dargestellt. Mit einem Maximalwert von über $90 \%$ in Reflexionsgeometrie und einem Minimalwert von $6 \%$ für $T_{0}$ wird die Resonanzbandbreite (FWHM) für diese Probe auf etwa 0,6 nm bestimmt. Mit einer Resonanzwellenlänge von 694,24 nm liegt die Wellenlänge nur 0,6 $\AA$ von der angestrebten Rubinlaser-Wellenlänge 694,3 nm entfernt. Zudem würde die gemessene Resonanzbandbreite die ca. $300 \mathrm{GHz}(\widehat{=} 0,48 \mathrm{~nm})$ breite $R_{1}$-Laserlinie des Rubinlasers vollständig reflektieren.

Darüber hinaus ist in den gezeigten Spektren eine deutlich sichtbare Oszillation zu verzeichnen, welche bedingt durch die Substratschicht eine Interferenzerscheinung (Dünnschicht-Interferenz) darstellt (STENZEL 1996) und sich mit dem eigentlichen Resonanzspektrum überlagert (s. Abschnitt 4.4. Wie man anhand der Summendarstellung $S_{0}$ sieht, hebt sich die Oszillation jedoch nicht an allen Stellen des Spektrums auf. Vielmehr kann eine unterschiedliche Oszillationsfrequenz im $T_{0^{-}}$ 
und $R_{0}$-Spektrum beobachtet werden, die sich z. B. an der Stelle 693,2 nm gerade aufhebt. Insgesamt deutet dies für das jeweilige Spektrum auf eine unterschiedliche optische Weglänge der interferierenden Teilstrahlen hin, deren Ursache bis zum Abschluss dieser Arbeit nicht geklärt werden konnte. Eine mögliche bzw. denkbare Erklärung könnte durch das vor der CCD angebrachte Schutzglas gegeben werden, welches zwar bei allen Kameras (selben Typs) gleich dick sein sollte, jedoch bei leicht schrägem Lichteinfall unterschiedliche Interferenzoszillationen hervorrufen kann. Die Mittelung der Intensitäten des Summenspektrums über den dargestellten Wellenlängenbereich ergibt den Wert 1 mit einer Standardabweichung von $6 \%$. Damit können die Absorptions- und Streuverluste bei der Bestrahlung der Probe DGWS/Nb487/40 im Rahmen der Messgenauigkeit nicht nachgewiesen werden. Nach Glättung der Spektren, d. h. einer Mittelwertbildung über 7 Messpunkte, ergibt sich in Resonanz eine Reflektivität von etwa $85 \%$, in Transmission ein Wert von rund $10 \%$. Der Fehler der Intensitäten wird dabei auf $2 \%$ geschätzt.

Ergänzend zu den gemessenen Daten zeigt Abb. 4.12b die berechneten Spektren (RCWA anhand der vorliegenden Strukturparameter der Probe DGWS/Nb487/40. Dabei ist der Wert für die Brechzahl des Wellenleitermaterials $\mathrm{Nb}_{2} \mathrm{O}_{5}$ von 2,23 (gemessen) auf 2,2377 verschoben worden, wodurch sich die berechnete Resonanz-

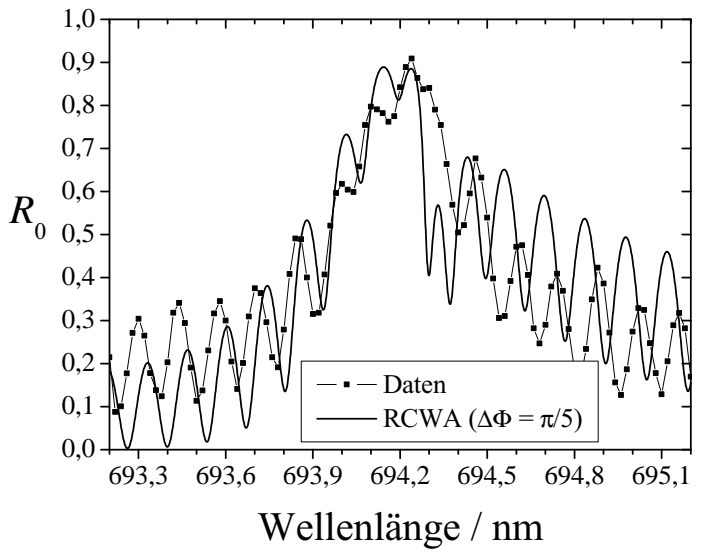

Abb. 4.13: Mittels RCWA über den Gitterversatz angepasstes $R_{0}$-Spektrum der Probe DGWS/Nb487/40 (vgl. Abb. 4.12. wellenlänge an die gemessene Wellenlänge anpasst. Auffällig ist, dass die theoretische Resonanzbandbreite (FWHM) von $0,2 \mathrm{~nm}$ gegenüber der gemessenen Bandbreite von $0,6 \mathrm{~nm}$ deutlich kleiner ausfällt. Mit hoher Wahrscheinlichkeit kann diese Diskrepanz auf die Strukturinhomogenität der nicht näher untersuchten Probenoberfläche zurückgeführt werden. Eine mögliche Erklärung findet sich durch ein evtl. anisotropes Schichtwachstum während des Beschichtungsprozesses der DGWS-Proben: Bei Schichtdicken von $487 \mathrm{~nm}$ (Probe DGWS/Nb487/40) 


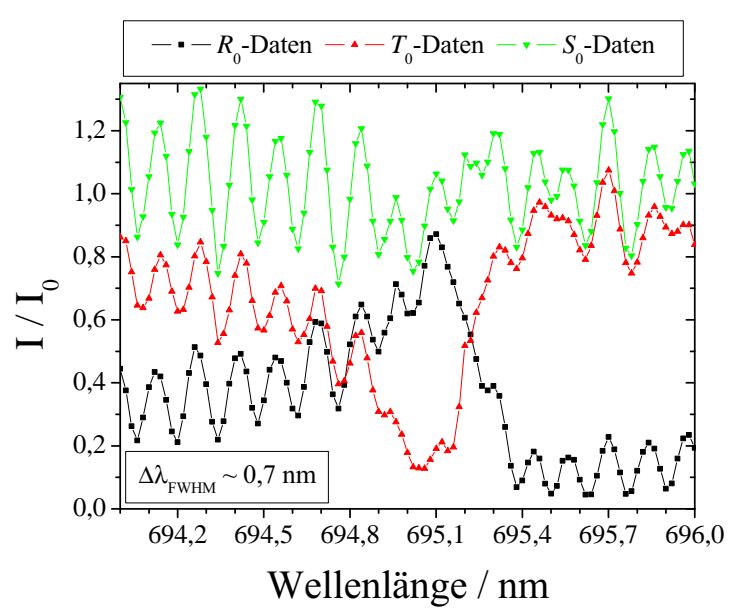

(a) Gemessenes $R_{0^{-}}, T_{0}$ und $S_{0^{-}}$ Spektrum der Probe DGWS/Nb551/40.

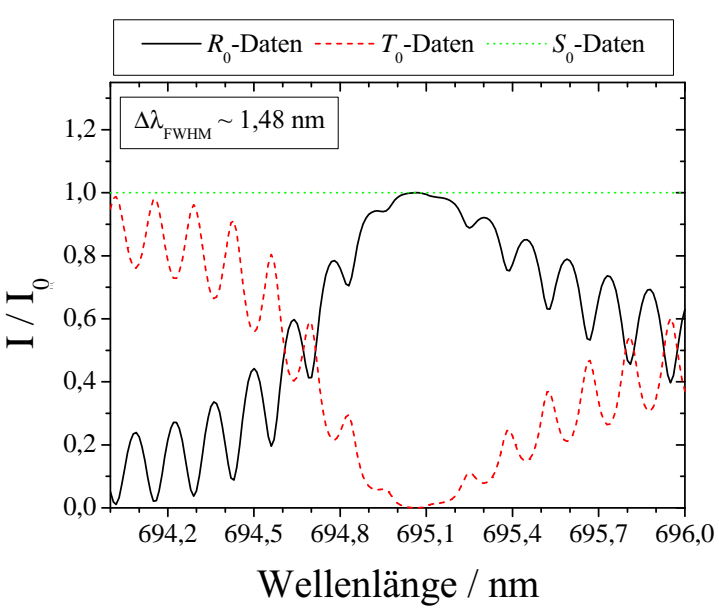

(b) Berechnetes $R_{0^{-}}, T_{0}$ und $S_{0^{-}}$ Spektrum der Probe DGWS/Nb551/40.

Abb. 4.14: Gemessene (a) sowie berechnete (b) $R_{0^{-}}, T_{0}$ und $S_{0^{-}}$Spektren der Probe DGWS/Nb551/40 durch Anregung mit TM-polarisiertem Licht.

bzw. $551 \mathrm{~nm}$ (Probe DGWS/Nb551/40) im Vergleich zur Gittertiefe von $40 \mathrm{~nm}$ ist eine laterale Verschiebung der Gitterstege oberhalb der Schicht, bezüglich der Gitterstege unterhalb der Schicht, denkbar [vgl. Abb. 2 bei BRAZAS u. a. (1995)]. Mit diesem relativen Gitterversatz ändert sich jedoch das Beugungsverhalten der DGWS, d. h. die Symmetrie zwischen +1 . und -1 . Beugungsordnung einer unter $0^{\circ}$ einfallenden Welle wird aufgehoben (AvRutsky u. a. 1989, LI 1995). Beide Ordnungen regen nun unterschiedliche Resonanzen an (s. Abschnitt 2.5), können jedoch spektral so dicht beieinander liegen, dass sie u. U. nicht vollständig aufgelöst werden können. Wie in Abb. 4.13 dargestellt ist, kann die Simulation bereits mit einem relativen Gitterphasenversatz von $\pi / 5$ an das gemessene Resonanzspektrum so angepasst werden, dass eine bessere Übereinstimmung zwischen Theorie und Experiment erzielt wird (BADER u. a. 2007; DELMDAHL u. a. 2007).

Abb. 4.14a zeigt das ausgewertete Reflexions- und Transmissionsspektrum $\left(R_{0}\right.$ bzw. $\left.T_{0}\right)$ sowie die Summendarstellung der Spektren $S_{0}$ der mittels TM. polarisiertem Laserlicht unter $0^{\circ}$ angeregten Probe DGWS/Nb551/40 für den Wellenlängenbereich 694-696 nm. Wie bei der Probe DGWS/Nb487/40 sind innerhalb der Spektren deutliche Oszillationen (Substratschicht-Interferenzen) zu erkennen. Mit der Mittelung von $S_{0}$ über das gesamte Wellenlängenspektrum nimmt $S_{0}$ den 
Wert $1 \pm 0,1$ an. Dies deutet daraufhin, dass bei der Probe DGWS/Nb551/40 evtl. auftretende Absorptions- und Streuverluste $(A+S)$ vernachlässigt oder anderenfalls im Rahmen der Messgenauigkeit nicht nachgewiesen werden können. Die Resonanzwellenlänge, welche in $T_{0}$ und $R_{0}$ minimal gegeneinander verschoben ist, beträgt im Mittel etwa 695,08 nm. Damit liegt die Resonanzwellenlänge knapp $8 \AA$ von der angestrebten Rubinlaser-Wellenlänge entfernt. Die maximale Reflexion der Probe wird auf $87 \pm 10 \%$ (bzw. $78 \pm 2 \%$ nach Glättung) und das Transmissionsminimum auf etwa $13 \pm 10 \%$ (bzw. $16 \pm 2 \%$ nach Glättung) bestimmt. Das dargestellte Summensignal $S_{0}$ zeigt aufgrund der hohen Gleichphasigkeit der $T_{0^{-}}$ und $R_{0}$-Spektren eine sehr ausgeprägte Oszillation. Dies drückt sich in der hohen Standardabweichung des gemittelten $S_{0}$-Signals von knapp $15 \%$ aus; wobei $S_{0}$ im Mittel wieder den Wert 1 annimmt.

Darüber hinaus sind in Abb. 4.14b die berechneten Spektren $T_{0}$ bzw. $R_{0}$ (RCWA für die Probe DGWS/Nb551/40 dargestellt. Die Anpassung der Resonanzwellenlänge erfolgt hier ebenfalls durch die Anhebung des Brechungsindexes der hochbrechenden $\mathrm{Nb}_{2} \mathrm{O}_{5}$-Schicht von gemessenen 2,25 auf 2,2515. Wie zuvor kommt als mögliche Ursache für die Diskrepanz zwischen gemessener und berechneter Resonanzbandbreite (FWHM) ein anisotropes Schichtwachstum der 551 nm dicken Schicht in Frage. Eine entsprechend angepasste $R_{0}$-Simulation der DGWS Probe

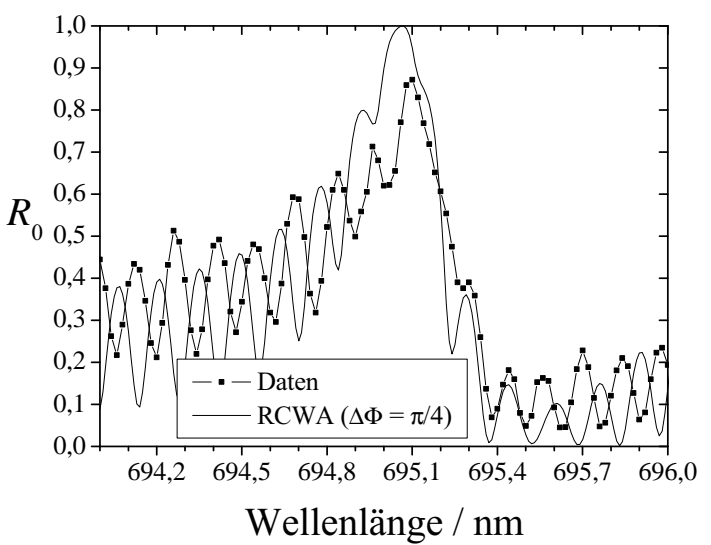

Abb. 4.15: Mittels RCWA über den Gitterversatz angepasstes $R_{0}$-Spektrum der Probe DGWS/Nb551/40 (vgl. Abb. 4.14). ist in Abb. $4.15 \mathrm{zu}$ sehen. Unter der Annahme eines relativen Gitterphasenversatzes von $\pi / 4$ und einer Erhöhung des Füllfaktors des Oberflächengitters um $30 \%$ auf 0,65 kann das simulierte Spektrum besser an das gemessene Resonanzspektrum angepasst werden. Insbesondere der erhöhte Füllfaktor zugunsten des wellenleitenden Materials ist nach der Aufspaltung der Resonanz durch die über den Gitterversatz erzeugte Phasenverschiebung für die bessere Übereinstimmung - vor allem in der Breite - verantwortlich. 


\subsubsection{Durchstimmbares Resonanzverhalten bei Temperaturerhöhung}

Zur Charakterisierung der temperaturspezifischen Probe DGWS/Hf331/18 bei der Helium-Neon-Laserwellenlänge $632,816 \mathrm{~nm}$ wird das Farbstofflasersystem durch einen entsprechenden Farbstoffwechsel angepasst. Um die Temperatur dieser Probe regeln zu können, wird eine speziell angefertigte Heizvorrichtung verwendet, die ein Aufheizen der Probe ermöglicht. Wie in Abb.4.16 zu sehen ist, besteht die Vorderseite der Vorrichtung, d.h. die zum Laserstrahl hingewandte Seite, aus einer beheizbaren Kupferplatte, auf der sich zwei metallene Halteklemmen zur Arretierung

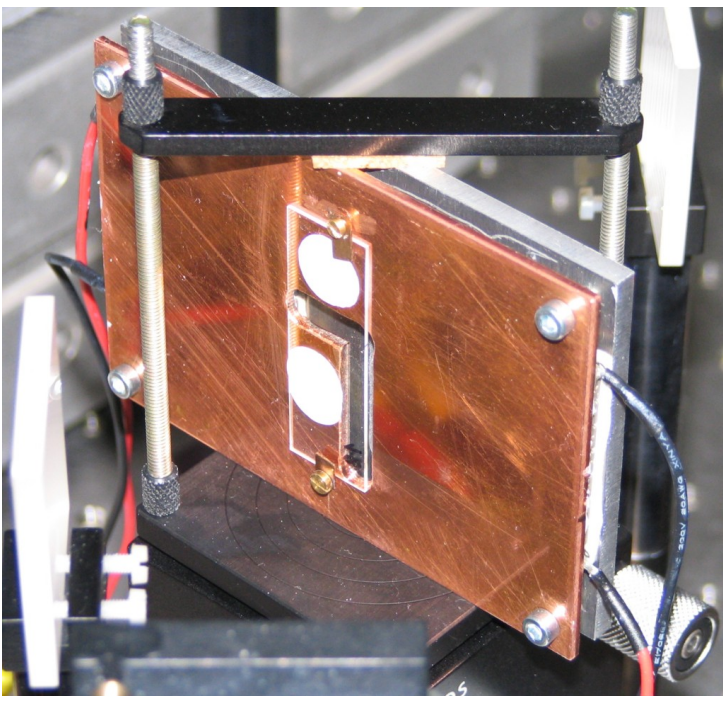

Abb. 4.16: Probenhalter mit PeltienElementen. der Probe befinden. Die Rückseite der Heizvorrichtung wird von einer Aluminiumplatte gebildet. Zwischen den beiden Platten sind zwei thermoelektrische Module, sog. Peltier-Elemente, vom Typ TEC1-12705 (Maße: $40 \times 40 \times 4 \mathrm{~mm}^{3}$ ) so integriert, dass der Temperaturgradient während des Heizvorgangs stets in Richtung der Kupferplatte zeigt und somit an deren Oberfläche eine zur Umgebung höhere Temperatur erzeugt werden kann. Bei einer angelegten Gleichspannung von 15,4 V beträgt die maximale Stromaufnahme eines Elements 4,6 A. Damit ist eines der beiden PELTIER-Elemente in der Lage, eine Temperaturdifferenz $\Delta \mathrm{T}$ von über $60^{\circ} \mathrm{C}$ zu erzeugen, was insgesamt ein Aufheizen der Probe auf über $+90^{\circ} \mathrm{C}$ ermöglicht. Die Gesamtmaße der Heizvorrichtung betragen in der Höhe, Breite und Tiefe $7 \times 10 \times 1 \mathrm{~cm}^{3}$.

Um eine optimale Wärmekopplung zwischen der Kupferplatte und der Probe zu gewährleisten, liegt die Probe mit der Substratseite vollständig auf der Platte. Zudem wird der Zwischenraum durch eine Wärmeleitpaste (runde weiße Flecken) ausgefüllt, welche den Wärmetransport zusätzlich optimieren soll. Neben den nur durch die Stromkabel angedeuteten Peltier-Elementen ist in Abb. 4.16 auch der Prismentisch zu sehen, welcher die Heizvorrichtung mithilfe einer Strebe fest- 
hält und über den sich die Probe zum einfallenden Laserstrahl ausrichten lässt. Über den ausgefrästen Teil (umgedrehte L-förmige Bohrung) der Heizvorrichtung kann die 0,5 mm hohe Gitterfläche über die volle Breite der Probe ausgeleuchtet werden. Am linken unteren bzw. rechten oberen Bildrand sind zudem die (keilförmigen) Strahlteiler zu sehen, die vor bzw. hinter der Probe positioniert sind (s. Abb. 4.11).

Nach der Justierung der Probe DGWS/Hf331/18 auf $0^{\circ}$ Einfallswinkel wird das Resonanzverhalten der Probe zunächst ohne Aufheizen bei Zimmertemperatur (etwa $21^{\circ} \mathrm{C}$ ) bestimmt. Im Anschluss an diese Messung wird der Strom zum Heizen der PELTIER-Elemente über ein geeignetes Labornetzteil auf 0,8 A geregelt, so dass die Probe über die Kupferplatte aufgeheizt wird. Nach einer Heizzeit von etwa zwei Stunden hat sich bei diesem Strom die Temperatur der Probe konstant bei etwa $35^{\circ} \mathrm{C}$ eingestellt, so dass sich das System aus Heizvorrichtung und Probe in einem thermischen, und die Probe zur umgebenden Luft in einem thermodynamischen, Gleichgewicht befinden (s. u.). Zur Bestimmung der Temperaturen wird ein handelsübliches Digital-Multimeter der Firma PEAKTECH ${ }^{\circledR}$ verwendet, welches die Temperatur ganzzahlig mit einer Genauigkeit von $\pm 1 \%$ darstellt.

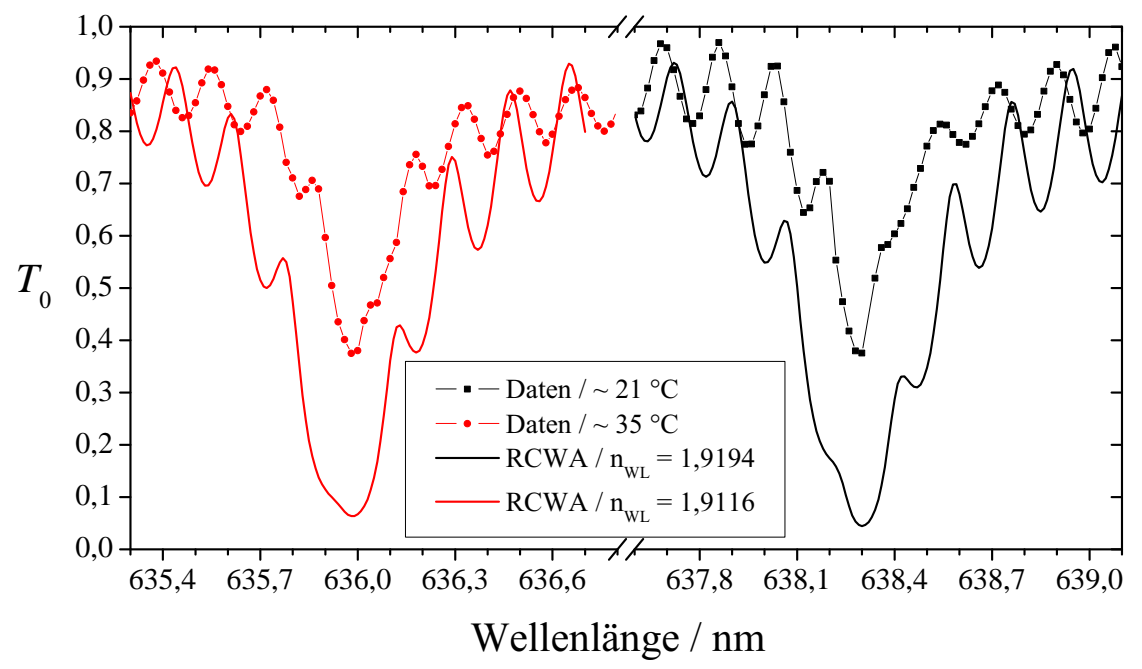

Abb. 4.17: Gemessenes (Daten) und berechnetes RCWA Transmissionsspektrum der Probe DGWS/Hf331/18 bei unterschiedlichen Temperaturen bzw. unterschiedlichen Wellenleiterbrechzahlen $n_{\mathrm{wL}}$. Die Verschiebung $\Delta \lambda / \Delta \mathrm{T}$ von $-0,164 \mathrm{~nm} \cdot \mathrm{K}^{-1}$ entspricht einem thermo-optischen Koeffizienten von $\Delta n_{\mathrm{WL}} / \Delta \mathrm{T}=-5,57 \cdot 10^{-4} \mathrm{~K}^{-1}$. 
Abb. 4.17 zeigt das Transmissionsspektrum der mittels TM-polarisiertem Laserlicht angeregten Probe DGWS/Hf331/18 bei den Temperaturen $21^{\circ} \mathrm{C}$ und $35^{\circ} \mathrm{C}$. Während das Transmissionsminimum bei Zimmertemperatur bei der Wellenlänge $638,3 \mathrm{~nm}$ liegt, stabilisiert sich die Resonanzwellenlänge nach der Temperaturerhöhung bei 636,0 nm. Für beide Resonanzwellenlängen kann eine spektrale Resonanzbandbreite von $0,3 \mathrm{~nm}$ bestimmt werden. Der hohe Minimalwert von rund $38 \pm 10 \%$ (bzw. $50 \pm 2 \%$ nach Glättung) in beiden $T_{0}$-Spektren ist vermutlich auf die unzureichende - zu diesem Zeitpunkt jedoch ausschließlich zur Verfügung stehende - Strahlverkleinerung durch ein invertiertes $7 \times$ Laseraufweitungssystem der Firma Linos zurückzuführen. Dieses arbeitet nach dem Prinzip des GALILEI-Fernrohrs und besteht aus einer konvexen Linsengruppe sowie einer konkaven Linse. Mithilfe dieser Optik soll der ca. $3 \mathrm{~mm}$ breite Laserstrahl eigentlich für die rund $0,5 \mathrm{~mm}$ schmalen Gitterstreifen der DGWS-Probe hinreichend verkleinert werden. Bei der Ausrichtung des minimalen Strahldurchmessers mittels Fokusrad zeigt sich jedoch, dass aufgrund der nahe an der Austrittsöffnung stattfindenden Fokussierung und der damit verbundenen Divergenzerhöhung eine Kollimation des Strahls nicht möglich ist. Somit lässt sich der Strahldurchmesser nur unzureichend verkleinern, wodurch einige Teile der einfallenden Welle die Probe ohne Gittereinfluss passieren und damit nicht zur Anregung der Resonanz beitragen.

Zusätzlich zu den gemessenen Daten sind in Abb. 4.17 die RCWA-Spektren dargestellt. Die Anpassung der berechneten Spektren geschieht über den komplexen Brechungsindex des Wellenleitermaterials $\tilde{n}_{\mathrm{wL}}$ mit $\kappa_{\mathrm{wL}}=1,8 \cdot 10^{-4}$ [vgl. (2.11] ]. Durch eine Verschiebung der reellen Brechzahl um $\Delta n_{\mathrm{wL}}=-0,0078$, was einer Indexabsenkung von etwa 3,5\% entspricht, kann die temperaturbedingte Verschiebung angepasst werden. Eine zusätzliche Drift der Resonanzwellenlänge bei den Temperaturen $33^{\circ} \mathrm{C}$ und $34^{\circ} \mathrm{C}$ konnte durch weitere Messungen bestätigt werden (Daten nicht gezeigt). Die gezeigte Verschiebung der Resonanzbandbreite um $-2,3 \mathrm{~nm}$ bei einer Temperaturdifferenz $\Delta \mathrm{T}$ von rund $14 \mathrm{~K}$, d. h. $\Delta \lambda / \Delta \mathrm{T}=-0,164 \mathrm{~nm} \cdot \mathrm{K}^{-1}$, entspricht einem thermo-optischen Koeffizienten von $\Delta n_{\mathrm{wL}} / \Delta \mathrm{T}=-5,57 \cdot 10^{-4} \mathrm{~K}^{-1}$.

Physikalisch lässt sich diese Drift durch die Verdrängung des Wassers aus der porösen $\mathrm{HfO}_{2}$-Schicht erklären: Durch die Zuführung von thermischer Energie gelingt es, die Bindung (Adhäsion) der Wassermoleküle mit dem $\mathrm{HfO}_{2}$-Material aufzubrechen. Je nach Temperatur kann so ein Teil des Wassers über die offene Poren- 
struktur der Schicht entweichen (Austreibung). In den Poren befindet sich nun verhältnismäßig mehr Luft - also vornehmlich Stickstoff- und Sauerstoffmoleküle - als Wassermoleküle. Aufgrund des im Vergleich zur umgebenden Luft höheren Brechungsindex des Wassers von 1,33 (Luft 1,000) verringert sich dadurch der mittlere Brechungsindex der Schicht. Wie in Abschnitt 2.4.2 theoretisch gezeigt, hat dies eine Verschiebung der Resonanzwellenlänge bzw. des -winkels zur Folge. Da es sich um einen reversiblen Prozess handelt, wird bei entsprechender Abkühlung der Probe eine entgegengesetzte Drift der Resonanzwellenlänge beobachtet.

Die Temperaturänderung $\Delta \mathrm{T}$ der Probe ist an thermodynamische Prozesse gekoppelt, wobei in erster Näherung von einer isobaren Zustandsänderung ausgegangen werden kann; d. h. eine Ausdehnung des Mischvolumens $\Delta \mathrm{V}$ aus Wasserdampf und Luft ist die Folge: $\Delta \mathrm{T} \sim \Delta \mathrm{V}$. Aufgrund der langen Wartezeit während des Heizvorgangs (konstante Zufuhr elektrischer Energie) stellt sich ein neues thermisches und thermodynamisches Gleichgewicht zwischen Probe und Umgebung ein. Insbesondere der hydrostatische Gleichgewichtszustand wird jedoch maßgeblich von den hygroskopischen Eigenschaften des $\mathrm{HfO}_{2}$ bestimmt (WERNECKE 2003). Die spezifischen Eigenschaften der Schicht, wie Größe, Anzahl und Geometrie der Zwischenräume, welche das Wasseraufnahmevermögen stark beeinflussen, sind im Rahmen dieser Arbeit jedoch nicht näher untersucht worden.

Unter Annahme einer Stoffporosität von $10 \%$ (EBERT und EBERT 2002) kann über die Brechungsindexänderung $\Delta n_{\mathrm{wL}}=0,0078$ die Änderungsrate des Wassergehalts abgeschätzt werden. Für die Temperaturänderung von $\Delta \mathrm{T}=+14 \mathrm{~K}$ beträgt diese bei einem (in erster Näherung) linearen Verlauf etwa $-24 \%$. Dies bedeutet, dass bereits bei dieser Temperaturdifferenz knapp 1/4 des Wassers aus den Poren getrieben wird. Da jedoch keine hygroskopischen Eigenschaften der $\mathrm{HfO}_{2}$-Schicht bekannt sind und auch keine experimentellen Daten zum Wassergehalt der Schicht vorliegen, darf diese Näherung bezweifelt werden. Eine andere Annahme geht hingegen von einer Porosität von $3 \%$ aus, was bei einer vollständig wasserfreien Probe (Probentemperatur $100^{\circ} \mathrm{C}$ ) zu einer maximalen Verschiebung der Resonanzwellenlänge von 3,5 nm führt (LEITEL u. a. 2007). Doch auch bei diesem Ansatz kann lediglich festgestellt werden, dass die berechnete und gemessene Drift in derselben Größenordnung liegen - eine vollständige Klärung der gemessenen Drift scheitert durch das Fehlen von zusätzlichen Materialeigenschaften ebenso. 


\subsection{Ultrakurzpuls-Analyse der Leckwelle mittels Autokorrelator}

Um die zeitlichen Pulslängen (Pulsdauer) ultrakurzer Laserpulse unter Resonanzbedingungen $\mathrm{zu}$ untersuchen, werden die Proben DGWS/Ta150/24 und DGWS/Ta150/40 mithilfe von FemtosekundenLaserpulsen angeregt und deren Reflexionssignal $\left(R_{0}\right)$ mit einem Autokorrelator erfasst. Innerhalb des Experiments wird das Autokorrelationssignal zunächst über ein Display am Oszilloskop graphisch ausgegeben und anschließend zur computergestützten Auswertung gespeichert. Geeignete Laserpulse mit Pulslängen von über hundert Femtosekunden werden mit dem bereits in Abschnitt 4.2.2 spezifizierten Titan:Saphir-Laser vom Typ Tsunami (Spectra Physics) erzeugt. Abb. 4.18 zeigt die

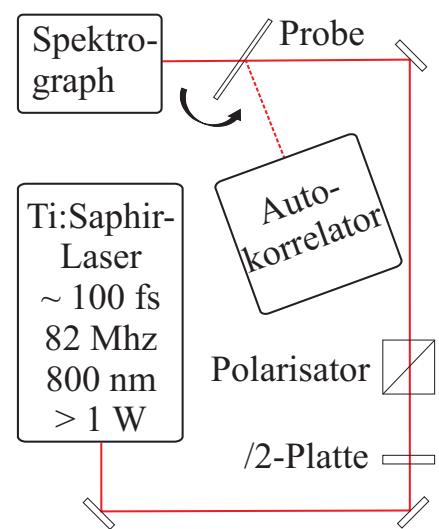

Abb. 4.18: Messanordnung mit dem Titan:Saphir-Lasersystem zur Vermessung von zeitlichen Pulslängen. Messanordnung zur Charakterisierung der Proben DGWS/Ta150/24 bzw. DGWS/Ta150/40 mit dem Ti:Saphir-Lasersystem. Durch die Kombination von $\lambda / 2$-Plättchen und Polarisator lässt sich der emittierte Laserstrahl des moden-gekoppelten Lasers in seiner Polarisation und Leistung einstellen. Zur Überprüfung der Wellenlänge des TE-polarisierten Laserstrahls wird ein Spektrograph direkt hinter dem Probentisch positioniert. Über den drehbaren Probentisch, welcher eine Winkelgenauigkeit von etwa $\pm 1 \%$ zulässt, wird der Resonanzwinkel für die jeweilige Probe eingestellt. Für TE-polarisiertes Licht der Wellenlänge $800 \mathrm{~nm}$ beträgt der Winkel danach -31, $5^{\circ}$ (DGWS/Ta150/24) bzw. -33,8 (DGWS/Ta150/40). Zur hinreichenden Bestrahlung der Probe DGWS/Ta150/24 wird ein invertiertes $7 \times$ Laseraufweitungssystem der Firma LiNOs verwendet.

Der Intensitätsautokorrelator vom Typ PulseScope (APE - AngewAndte PhySIK \& Elektronik GmbH) arbeitet nach dem Prinzip eines MichelsonInterferometers (s. Abb. A.5): Das einfallende Licht der Intensität $I(t) \sim|E(t)|^{2}$ wird mittels Strahlteiler aufgespalten und anschließend, nach Durchlaufen unterschiedlicher Wegstrecken $\Delta s$, mit einer zeitlichen Verzögerung $\tau=\Delta s / \mathrm{c}$ im 
Strahlteiler wieder zusammengeführt. Das durch die Überlagerung entstehende Interferenzsignal $I(t, \tau)$ stellt somit eine Superposition der einfallenden Welle mit sich selbst dar. Aufgrund des innerhalb des Autokorrelators verbauten SHG. Kristalls (s. Skizze Abb. A.5 wird ein frequenzverdoppeltes Interferenzsignal $I_{\text {SHG }}(t, \tau)$ erzeugt (Autokorrelator 2. Ordnung), welches nach Filterung der Grundwelle ausschließlich vom Quadrat des Interferenzsignals $I(t, \tau)$ abhängt (DEMTRÖ-

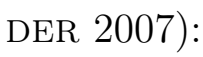

$$
I_{\underline{\mathrm{SHG}}}(t, \tau) \sim I^{2}(t, \tau)=\left[I_{1}(t)+I_{2}(t+\tau)\right]^{2}
$$

Das vom Autokorrelator über ein Zeitfenster $T_{F}$ aufgezeichnete Korrelationssignal SAKF lässt sich durch eine sog. Autokorrelationsfunktion - kurz AKF - darstellen, welche ausschließlich von der zeitlichen Verzögerung (Korrelationszeit) $\tau$ abhängt:

$$
S_{\mathrm{AKF}}(\tau)=\lim _{T_{F} \rightarrow \infty} \frac{1}{2 T_{F}} \int_{-T_{F}}^{+T_{F}} I_{\text {[SHG }}(t, \tau) \mathrm{d} t .
$$

Für den freien Laserstrahl mit einer zeitlichen Pulsform nach GAUsS

$$
I(t)=I_{0} \cdot \mathrm{e}^{\frac{-t^{2}}{2 \cdot w^{2}}}
$$

- einer Amplitude $I_{0}$ und der Standardabweichung $w$ - ergibt sich nach Einsetzen in Formel 4.1 und 4.2 ein Detektorsignal:

$$
S_{\operatorname{AKF}}(\tau) \approx 4 \cdot \sqrt{\pi} \cdot I_{0}^{2} \cdot w \cdot \mathrm{e}^{\frac{-\tau^{2}}{4 \cdot w^{2}}} .
$$

Abb. 4.19a zeigt das mittels Autokorrelator aufgezeichnete, auf Eins normierte Signal (Daten) des Ti:Saphir-Laserstrahls (ohne Probe). Zusätzlich ist eine Anpassung der Messdaten durch eine Fitfunktion nach (4.4) dargestellt. Danach kann die 


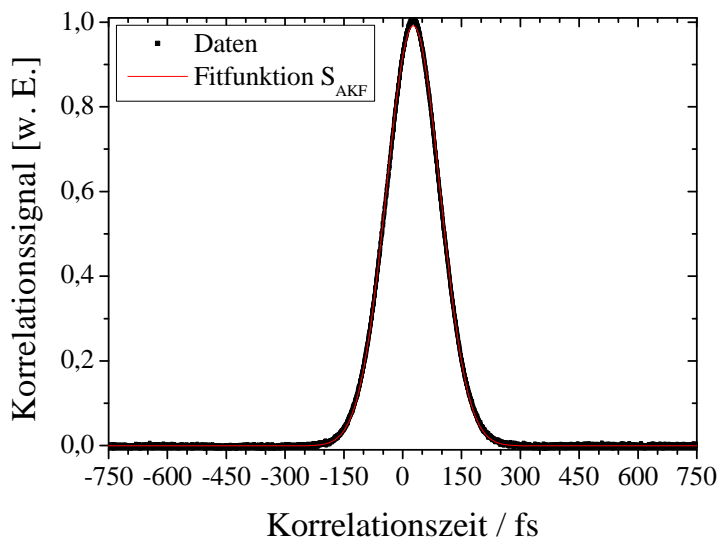

(a) Zeitabhängiges Korrelationssignal vom Laserpuls (ohne Probe).

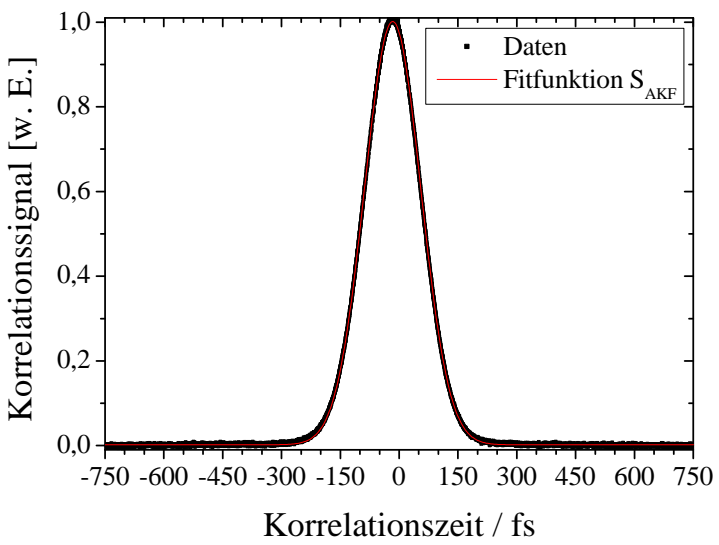

(b) Korrelationssignal der Probe DGWS/Ta150/24 (nicht-resonant).

Abb. 4.19: Mittels Autokorrelator aufgenommenes zeitabhängiges Korrelationssignal (Daten) (a) des freien Laserpulses und (b) des Rückreflexes der Probe DGWS/Ta150/24 (nicht-resonant). Die Anpassung der Messdaten erfolgt über die Fitfunktion SAKF.

Standardabweichung $w$ des Laserstrahls auf 48,6 fs bestimmt werden. Der Fitfehler beträgt bei dieser Ausgleichsrechnung $\pm 0,011$ fs. Über die Umrechnungsformel (DIELS und RUDOLPH 1996)

$$
\Delta t_{\overline{\text { FWHM }}}=2 \cdot \sqrt{2 \ln 2} \cdot w
$$

lässt sich die zeitliche Halbwertsbreite des Laserpulses $\Delta t_{\mathrm{FWHM}}$ auf rund 114 fs bestimmen. Darüber hinaus zeigt Abb. 4.19bden von der Probenoberfläche reflektierten Laserstrahl bei nicht-resonanter Anregung (TM) der Probe DGWS/Ta150/24. Die Auswertung der Daten ergibt eine Standardabweichung von 50,69 $\pm 0,01 \mathrm{fs}$, was nach (4.5) eine zeitliche Pulslänge $\Delta t \overline{\text { FWHM }}$ von etwa 119,36 $\pm 0,02$ fs ergibt.

Eine (raum-zeitliche) Pulsverlängerung oder Gruppenlaufzeitdispersion (engl. group delay dispersion - kurz GDD, welche beim Durchlaufen dispersiver Medien unter dem Einfluss der Gruppengeschwindigkeitsdispersion (engl. group velocity dispersion, GVD 2. Ordnung erzeugt wird (DEMTRÖDER 2007), kann bei einer optischen Weglänge von unter $2 \mathrm{~cm}$ (Produkt aus Substratbrechzahl und Proben- 
dicke) vernachlässigt werden. Dies wird bestätigt von Korrelationsmessdaten der Probe DGWS/Ta150/40 bzw. der Probe DGWS/Ta150/24, wonach in Transmission eine Pulsdauer $\Delta t_{\overline{F W H M}}$ von etwa $114,43 \pm 0,02 \mathrm{fs}$, respektive 120,31 $\pm 0,02 \mathrm{fs}$, ermittelt wird (Daten nicht gezeigt). Die Abweichungen der verschiedenen Pulslängen deuten jedoch auf einen zufälligen Fehler hin, welcher deutlich größer als der Fitfehler ist und für alle Korrelationssignale auf maximal $5 \%$ geschätzt wird.

Zur Bestimmung der Pulsdauer der Leckwelle muss die mittlere Ein- und Auskoppelzeit bzw. die optische Weglänge der ein- und ausgekoppelten Welle berücksichtigt werden. Nach Formel (2.57) kann mithilfe der zuvor bestimmten Resonanzbandbreiten $\Delta \lambda_{\text {FWHM }}$ (s. Abschnitt 4.2.2) die resonante Kopplungslänge $l_{\mathrm{R}}$ der Proben abgeschätzt werden. Danach ergibt sich für die Probe DGWS/Ta150/40 mit einer TE-Resonanzbandbreite von $4,6 \mathrm{~nm}$ ein $l_{\mathrm{R}}$ von etwa $20 \mu \mathrm{m}$ bzw. für die Probe DGWS/Ta150/24 mit einer TE-Resonanzbandbreite von $1,8 \mathrm{~nm}$ ein $l_{\mathrm{R}}$ von $51 \mu \mathrm{m}$.

Die Kohärenzlänge $l_{\mathrm{c}}$ des freien Femtosekunden-Laserpulses (ohne Probe) kann über die Kohärenzzeit $\tau_{\mathrm{c}}$ mit $l_{\mathrm{c}}=\mathrm{c}_{0} \cdot \tau_{\mathrm{c}} \approx \mathrm{c}_{0} \cdot \Delta \mathrm{t}_{\mathrm{FWHM}}$ auf $34 \pm 2 \mu \mathrm{m}$ geschätzt werden. Nach Aufspaltung der einfallenden Welle in die Teilwellen der Feldstärken $E_{\mathrm{F}}(t)$ (FRESNEL-Anteil) und $E_{\mathrm{R}}(t)$ (resonante Leckwelle) findet nach der Wiederauskopplung von $E_{\mathrm{R}}(t)$ für die Probe DGWS/Ta150/40 eine partiell kohärente bzw. für die Probe DGWS/Ta150/24 eine vollständig inkohärente Überlagerung mit dem Fresnel-Reflex statt. Die Gesamtintensität $I$ unter Resonanzbedingungen lässt sich somit in Abhängigkeit von der zeitlichen Kopplungslänge $\tau_{\mathrm{R}}=l_{\mathrm{R}} / \mathrm{c}$ darstellen:

$$
I\left(\tau_{\mathrm{R}}\right)=\left\langle E_{\mathrm{F}}(t) E_{\mathrm{F}}^{*}(t)\right\rangle+\left\langle E_{\mathrm{R}}(t) E_{\mathrm{R}}^{*}(t)\right\rangle+2 \operatorname{Re}\left\{\left\langle E_{\mathrm{F}}^{*}(t) E_{\mathrm{R}}\left(t+\tau_{\mathrm{R}}\right)\right\rangle\right\} .
$$

Das komplexe Argument innerhalb des letzten Terms in 4.6 ist ein Maßs für die kohärente Überlagerung von $E_{\mathrm{F}}(t)$ und $E_{\mathrm{R}}(t)$ und wird als komplexe Kreuzkorrelations- bzw. Kreuzkohärenzfunktion (KKF) bezeichnet (LAUTERBORN u. a. 1993). Unter der Annahme vollständiger Inkohärenz kann die KKF demnach vernachlässigt werden. 
Das in den Autokorrelator einfallende Signal $I_{1}(t)$ setzt sich dann aus dem FRESNEL-Anteil $I_{\mathrm{F}}(t)$ sowie dem resonanten Leckwellenanteil $I_{\mathrm{R}}(t)$ zusammen:

$$
I_{1}(t)=I_{\mathrm{F}}(t)+I_{\mathrm{R}}(t)
$$

respektive gilt für das innerhalb des Autokorrelators verzögerte Signal $I_{2}(t+\tau)$ :

$$
I_{2}(t+\tau)=I_{\mathrm{F}}(t+\tau)+I_{\mathrm{R}}(t+\tau)
$$

Nach Einsetzen in die Formeln 4.1) und (4.2) ergibt sich unter der Annahme von Gauss-Pulsen für das FREsneL- bzw. Leckwellensignal eine AKF SAKF, welche im Wesentlichen aus drei Termen besteht:

$$
S \underline{\underline{\mathrm{AKF}}} \approx I_{0, \mathrm{~F}}^{2} \cdot w_{\mathrm{F}} \cdot \mathrm{e}^{\frac{-\tau^{2}}{4 \cdot w_{\mathrm{F}}^{2}}}+I_{0, \mathrm{R}}^{2} \cdot w_{\mathrm{R}} \cdot \mathrm{e}^{\frac{-\tau^{2}}{4 \cdot w_{\mathrm{R}}^{2}}}+4 \cdot I_{0, \mathrm{~F}} \cdot I_{0, \mathrm{R}} \cdot \sqrt{\frac{w_{\mathrm{F}}^{2} \cdot w_{\mathrm{R}}^{2}}{2 \cdot\left(w_{\mathrm{F}}^{2}+w_{\mathrm{R}}^{2}\right)} \cdot \mathrm{e}^{\frac{-\tau^{2}}{2 \cdot\left(w_{\mathrm{F}}^{2}+w_{\mathrm{R}}^{2}\right)}}}
$$

Der erste Summand gibt dabei das Korrelationssignal des direkt von der Probenoberfläche reflektierten Laserstrahlanteils, d. h. den FRESNEL-Reflex, wieder. Demzufolge entspricht der zweite Term dem Korrelationssignal des resonant ein- und in Reflexion auskoppelnden Teils des Laserstrahls (Leckwelle). Der dritte Summand hat die Form eines Mischterms aus beiden Reflexen.

Abb. 4.20 zeigt das aufgenommene und normierte AKF-Signal bei resonanter Bestrahlung der Proben DGWS/Ta150/24 und DGWS/Ta150/40 mit TE polarisiertem Laserlicht. Darüber hinaus ist die Fitfunktion $S_{\text {AKF }}$ nach Formel 4.9 ) mit den entsprechenden Termen - hier durch FrESnEL, Leckwelle und Mischterm gekennzeichnet - dargestellt. Die Anpassung von SAKF an das Messsignal geschieht durch die freien Parameter $I_{0, \mathrm{~F}}$ und $I_{0, \mathrm{R}}$ sowie $w_{\mathrm{F}}$ und $w_{\mathrm{R}}$, welche nach der Methode der kleinsten Quadrate mithilfe des LEvEnBERG-MARQUARDT-Algorithmus (LEVENBERG 1944; MARQUARDT 1963) iterativ an die Messdaten angepasst werden. Aus den ermittelten Werten für $w_{\mathrm{F}}$ und $w_{\mathrm{R}}$ kann nach Formel 4.5 die Pulsdauer 


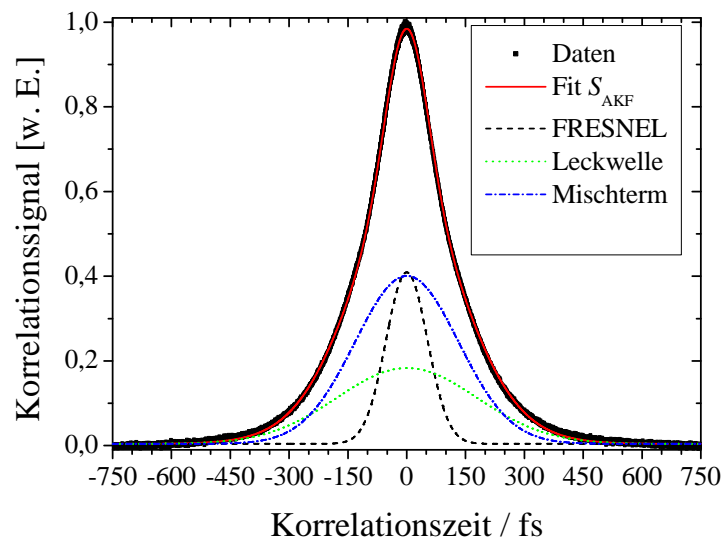

(a) Zeitabhängiges Korrelationssignal der Probe DGWS/Ta150/40.

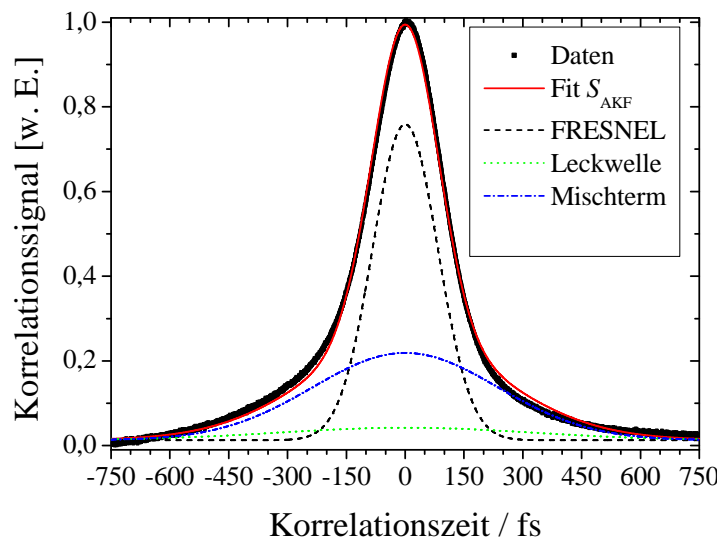

(b) Zeitabhängiges Korrelationssignal der Probe DGWS/Ta150/24.

Abb. 4.20: Mittels Autokorrelator aufgenommenes zeitabhängiges Korrelationssignal (Daten) (a) der Probe DGWS/Ta150/40 und (b) der Probe DGWS/Ta150/24. Die Anpassung der Messdaten erfolgt über die Fitfunktion $S$ AKF, wobei die einzelnen Korrelationsterme (FrESNEL, Leckwelle und Mischterm) separat dargestellt sind.

der Teilstrahlen bestimmt werden. So beträgt die Pulslänge der resonanten Leckwelle bei Bestrahlung der Probe DGWS/Ta150/40 rund 298 \pm 15 fs bzw. $539 \pm 27$ fs für die Probe DGWS/Ta150/24. Entsprechend kann die Pulsdauer des FRESNELStrahls auf $88 \pm 4$ fs (DGWS/Ta150/40) bzw. auf $139 \pm 7$ fs (DGWS/Ta150/24) bestimmt werden.

Unter der Annahme FOURIER-limitierter Gauss-Pulse lässt sich die zeitliche Pulslänge $\Delta t$ auf die spektrale Bandbreite $\Delta \lambda$ umrechnen (DIELS und RUDOLPH 1996): Über das Pulslänge-Bandbreite-Produkt für GAuss-Pulse

$$
\Delta t \cdot \Delta \lambda=\frac{2 \cdot \ln (2)}{\pi} \cdot \frac{\lambda^{2}}{c_{0}}
$$

kann für die Zentralwellenlänge $800 \mathrm{~nm}$ somit eine spektrale Resonanzbandbreite von 3,16 \pm 0,16 nm (DGWS/Ta150/40) bzw. 1,74 $\pm 0,09$ nm (DGWS/Ta150/24) ermittelt werden. Insbesondere die Bandbreite der Probe DGWS/Ta150/24 stimmt mit der in Abschnitt 4.2.2 gemessenen spektralen Resonanzbandbreite von 1,8 nm im Rahmen der Messgenauigkeit überein. Bei der Probe DGWS/Ta150/40 hingegen 
weicht der mittels Autokorrelator bestimmte Wert um etwa $30 \%$ vom Spektrometerwert ab. Diese Diskrepanz kann auf die Vernachlässigung der KKF innerhalb der Fitfunktion $S_{\mathrm{AKF}}$ zurückgeführt werden, wonach für diese Probe eine partiell kohärente Überlagerung von FRESNEL-Strahl und Leckwelle vorausgesagt worden ist (s. o.) und $S \widehat{A K F}$ der Vollständigkeit halber hätte angepasst werden müssen.

Über die Fitfunktion $S_{\text {AKF }}$ können darüber hinaus die Intensitäten $I_{0, \mathrm{~F}}$ und $I_{0, \mathrm{R}}$ der Teilwellen zum Zeitpunkt $t=0$ bestimmt werden. Mit der Bildung des Zeitintegrals über die entsprechenden Gauss-Pulse - vgl. 4.3 - kann die Energie $W$ der jeweiligen Teilstrahlen berechnet werden:

$$
W=\frac{1}{\sqrt{2 \pi}} \cdot I_{0} \cdot \int_{-\infty}^{+\infty} \mathrm{e}^{\frac{-t^{2}}{2 \cdot w^{2}}} \mathrm{~d} t
$$

Der Quotient aus $W_{\mathrm{R}}$ und $W_{\mathrm{F}}$ mit den entsprechenden Parametern $I_{0, \mathrm{R}}$ und $I_{0, \mathrm{~F}}$ bzw. $w_{\mathrm{R}}$ und $w_{\mathrm{F}}$

$$
\frac{W_{\mathrm{R}}}{W_{\mathrm{F}}}=\frac{I_{0, \mathrm{R}} \cdot w_{\mathrm{R}}}{I_{0, \mathrm{~F}} \cdot w_{\mathrm{F}}}
$$

gibt zudem das Verhältnis der Leckwellen- bzw. Kopplungsenergie und der Energie des FRESNEL-Strahls wieder. Danach kann für die Probe DGWS/Ta150/40 ein Verhältnis von etwa $6: 5$ berechnet werden, wonach ca. $55 \%$ des einfallenden Lichts der Leckwelle zugeordnet werden können. Mit einer Gewichtung von $2: 5$ liegt der Anteil der Kopplungsenergie der Probe DGWS/Ta150/24 hingegen unter $30 \%$. Dieser Betrag korreliert mit dem Verhältnis der Flächen der in Abb. 4.7 dargestellten Intensitätsspektren Laserpulse (ohne Probe) und $R_{0}$-Reflex. Die so ermittelten zeitlichen Pulslängen und Energieanteile werden zudem für eine Abschätzung des in Abschnitt 5.2 bestimmten Verstärkungsfaktors auf der Basis der Probe DGWS/Ta150/40 benutzt.

Der Einfluss einer Pulsverlängerung unter Resonanzbedingungen durch eine GDD kann aufgrund der Kopplungslängen im Mikrometerbereich vernachlässigt werden (KAPPEL u. a. 2004a). 


\subsection{Diskussion und Ergebnisse}

Die experimentellen Ergebnisse, welche bereits in den vorangegangen Abschnitten dargestellt worden sind, werden am Ende dieses Abschnitts noch einmal zusammengefasst und hinsichtlich eines möglichen Anwendungspotentials der DWG als dielektrischer Spiegel und/oder optischer Filter diskutiert. Zuvor werden noch offene Fragen aus den bisherigen Abschnitten geklärt. Dabei wird u. a. der Einfluss der Laserlinienbreite auf die Resonanzbandbreite erörtert sowie auf das Erscheinen bzw. Ausbleiben der bereits angesprochenen Oszillationen näher eingegangen.

Aufgrund der Tatsache, dass sämtliche Laserlinien der verwendeten Laserquellen eine laserspezifische Linienbreite besitzen, stellen alle gemessenen $T_{0^{-}}$und $R_{0^{-}}$ Spektren eine Überlagerung (Faltung) der Laserlinie und der tatsächlichen Resonanzbandbreite dar (BOYE und Kostuk 2000; TISHCHENKO u.a. 2003; NIEDERER u.a. 2004). Um den Einfluss der Linienbreite auf die Resonanzbandbreite abzuschätzen, bedarf es einer Analyse der spektralen Breite der einzelnen Laserlinien. Bei einigen Laserlinien gelingt dies durch die Bestimmung der Linienbreite mit dem Échelle-Spektrometer vom Typ SpectraStar S150HR (SOLAR LASER Systems). Bei anderen jedoch reicht die spektrale Auflösung des Spektrometers nicht aus, die Linie hinreichend aufzulösen, oder aber das Spektrometer steht zum Zeitpunkt der Messung schlichtweg nicht zur Verfügung.

Am Beispiel der Proben DGWS/Hf30/40, DGWS/Hf30/18 und DGWS/Hf74/40 können die zur Charakterisierung der Proben verwendeten UV Laserlinien 266,0 nm und 354,7 nm (s. Abschnitt 4.2.1) jedoch abgeschätzt werden. Dazu wird auf die Spektrometerdaten der sog. Signal- und Hilfswelle zurückgegriffen, welche mittels Nd:YAG-Laser (selben Typs) in Kombination mit einem sog. optisch parametrischen Oszillator/Generator OPO OPG erzeugt werden. Die Signal- und Hilfswelle werden dabei über eine sog. Drei-Wellen-Wechselwirkung mithilfe eines nichtlinearen BBO-Kristalls, welcher mit einer sog. Pumpwelle der Wellenlänge 354,7 nm angeregt wird, erzeugt (LAUTERBORN u. a. 1993; EICHLER und EICHLER 2006). Die entsprechende Messanordnung des verwendeten OPOF Lasersystems (inkl. einer Verstärkereinheit - engl. optical parametric amplification, kurz OPA ist in der Arbeit von SELLE (2003) gezeigt. 
Abb. 4.21 zeigt die mittels Spektrometer aufgenommenen OPO-Mischwellen der Wellenlänge $\lambda=525,13 \mathrm{~nm}$ (Signalwelle) bzw. 1093,08 nm (Hilfswelle), welche mithilfe der Pumpwelle der Wellenlänge $354,7 \mathrm{~nm}$ entstehen. Danach beträgt die spektrale Linienbreite $\Delta \lambda_{\text {FWHM }}$ für die Signalwelle $37 \mathrm{pm}$ bzw. für die Hilfswelle 160 pm. Der Fehler der Linienbreiten ist von der Genauigkeit des Spektrometers abhängig, welcher herstellerseitig mit $\pm 3 \mathrm{pm}$ angegeben ist. Über die Umrechnung der

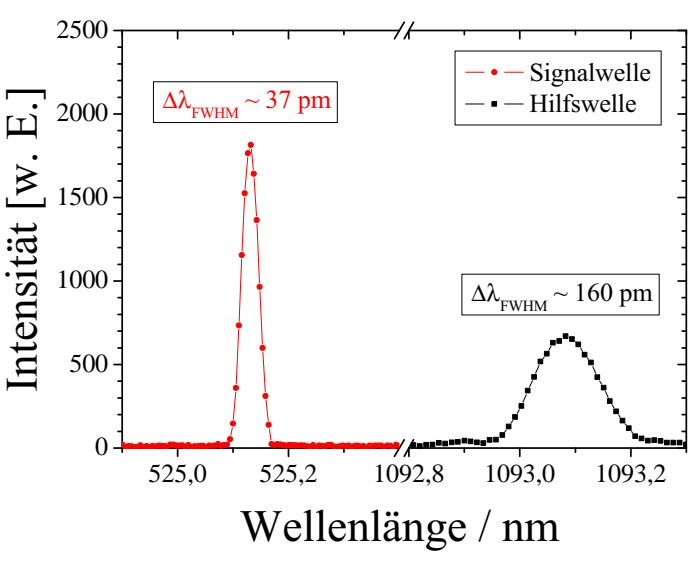

Abb. 4.21: Mittels Spektrometer aufgenommene OPO-Mischwellen: Signal- bzw. Hilfswelle. Wellenlänge $\lambda$ in die Frequenz $\nu$ lässt sich auch die zugehörige Wellenzahl $\tilde{\nu}$ berechnen:

$$
\tilde{\nu}=\frac{\nu}{\mathrm{c}_{0}}=\frac{1}{\lambda} .
$$

Danach entspricht die Linienbreite der Signal- bzw. Hilfswelle einer Frequenzlinienbreite in Wellenzahlen $\Delta \tilde{\nu}$ von jeweils $1,34 \mathrm{~cm}^{-1}$, wobei der Fehler der Signalwelle mit $0,11 \mathrm{~cm}^{-1}$ entsprechend größer ausfällt als der der Hilfswelle von rund $0,03 \mathrm{~cm}^{-1}$. Aus der Formel für den Drei-Wellen-Wechselwirkungsprozess

$$
\nu_{\mathrm{P}}\left(\nu_{\mathrm{S}}, \nu_{\mathrm{H}}\right)=\nu_{\mathrm{S}}+\nu_{\mathrm{H}}
$$

nach welcher sich die zugehörige Pumpfrequenz $\nu_{\mathrm{P}}$ unter Berücksichtigung der Frequenzen von Signal- und Hilfswelle $\nu_{\mathrm{S}}$ bzw. $\nu_{\mathrm{H}}$ ermitteln lässt, kann die Frequenzlinienbreite der Pumpwelle $\Delta \tilde{\nu}_{\mathrm{P}}$ auf die doppelte Breite von 2,68 $\pm 0,14 \mathrm{~cm}^{-1}$ bestimmt werden. Die Umrechnung in Wellenlängen ergibt demnach eine Laserlinienbreite der 3 . Harmonischen von etwa $34 \pm 2 \mathrm{pm}$.

Nach (2.59) kann der gemessenen Resonanzwinkel-(Halbwerts-)Breite eine spektrale Bandbreite zugeordnet werden, welche bei der TE-Anregung der Probe 
DGWS/Hf74/40 deutlich im zweistelligen Nanometerbereich liegt. Damit kann die Laserlinienbreite, welche um den Faktor 1000 spektral schmaler als die Resonanzbandbreite ist, vernachlässigt werden.

Die bei derselben Probe gemessene TM-Resonanzwinkelbreite von $0,6^{\circ}$ entspricht nach 2.59) einer spektralen Bandbreite von etwa $3 \mathrm{~nm}$, wonach auch hier der Einfluss der 3. Harmonischen bei der Verbreiterung der Resonanzbandbreite zu vernachlässigen ist.

Unter der Annahme, dass die Frequenzlinienbreite von 2,68 $\pm 0,22 \mathrm{~cm}^{-1}$ der DreiWellen-Wechselwirkung auch für die durch zweifache Frequenzverdopplung erzeugte 4. Harmonische des Nd:YAG Lasers gilt, entspricht die ermittelte Frequenzlinienbreite für die Wellenlänge $266 \mathrm{~nm}$ einer spektralen Linienbreite von etwa $19 \pm 2$ pm. Damit kann die Linienbreite innerhalb der in Abb. 4.3 gezeigten Winkelspektren der Proben DGWS/Hf30/18 und DGWS/Hf30/40 ebenfalls vernachlässigt werden.

Wie in Abschnitt 4.2 .3 erwähnt, kann das verwendete Échelle-Spektrometer mit einem spektralen Auflösungsvermögen von $\lambda / \Delta \lambda=3 \cdot 10^{4}$ die Laserlinienbreite des Farbstofflasers, welche laut Hersteller etwa 7 pm betragen soll, nicht auflösen. Abb. 4.22 zeigt das aufgenommene Laserlinienspektrum nahe der RubinlaserWellenlänge $694,3 \mathrm{~nm}$. Die darüber bestimmte spektrale Linienbreite von ca. $40 \pm 3$ pm ist jedoch als Überlagerung (Faltung) der tatsächlichen Linienbreite und einer sog. Unschärfefunktion oder Apparatefunktion, welche das Auflösungsvermögen des Échelle-Spektrometers bei dieser Wellenlänge unter Annahme einer GAUss-Verteilung darstellt, zu verstehen. Um die beiden Spektren zu entfalten, werden die sich überlagernden Funktionen $f$ und $g$ in den FOURIER-Raum transformiert $-\mathcal{F}(f)$ bzw. $\mathcal{F}(g)$ - und nach dem Faltungstheorem

$$
\mathcal{F}(f * g)=2 \pi \cdot \mathcal{F}(f) \cdot \mathcal{F}(g)
$$

durch einfache mathematische Division voneinander entkoppelt (LAUTERBORN u. a. 1993). 


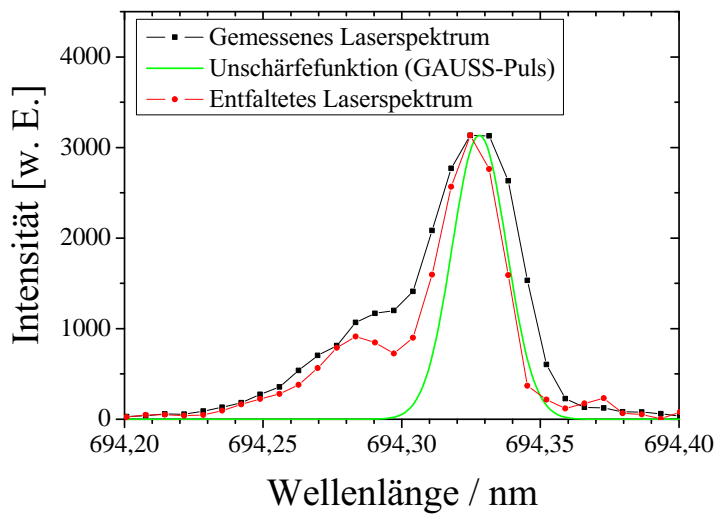

Abb. 4.22: Mittels Spektrometer aufgenommene Farbstofflaserlinie (schwarz). Zusätzlich ist die zur Entfaltung des Spektrums benötigte Unschärfefunktion (grün) als Gauss-Puls sowie das entfaltete Spektrum der Laserlinie (rot) dargestellt.
Abb. 4.22 zeigt die mittels Spektrometer aufgenommene Farbstofflaserlinie (schwarz) sowie die bei der Entfaltung verwendete Unschärfefunktion (grün). Diese hat die bereits angesprochene Gauss-Pulsform bei einer Halbwertsbreite FWHM von rund $23 \mathrm{pm}$. Das ebenfalls in Abb. 4.22 gezeigte entfaltete Linienspektrum (rot) besitzt nun eine Linienbreite von ca. $28 \pm$ $3 \mathrm{pm}$ und ist damit $30 \%$ schmaler als die ursprünglich gemessene Linienbreite. Dennoch, die aus der Entfaltung bestimmte Linienbreite von $28 \pm 3 \mathrm{pm}$ ist im Vergleich zu der vom Hersteller angegebenen Breite um den Faktor 4 größer und liegt nur eine Größenordnung unter den experimentell bestimmten Resonanzbandbreiten von 0,6 nm (Probe DGWS/Nb487/40) bzw. 0,7 nm (Probe DGWS/Nb551/40). Damit kann durch die Überlagerung von Laserlinienbreite und Resonanzbandbreite eine spektrale (bzw. angulare) Verbreiterung der Resonanz nicht ausgeschlossen werden. Bei der Betrachtung der Laserlinien in Abb. 4.22 fällt insbesondere auf, dass an der kurzwelligen Flanke der Linien mit einem Versatz von etwa $4 \mathrm{pm}$ zur Zentralwellenlänge (wenigstens) eine weitere LaserSchwingungsmode auftritt, welche im entfalteten Spektrum noch etwas deutlicher zu erkennen ist. Dies deutet möglicherweise auf eine unzureichende Justage der Laser-Resonatorspiegel bei den Messungen hin.

Ein weiterer - vor allem das Intensitätsverhalten beeinflussender - Faktor ist die in den Abschnitten 4.2.3.1 und 4.2.3.2 unter Resonanzbedingungen auftretende Oszillation, welche eine Interferenzerscheinung an der Substratschicht der verwendeten Proben darstellt. Nach dem Interferenzkriterium an dünnen Schichten muss die Kohärenzlänge $l_{c}$, welche über die Beziehung (BORN und WOLF 1999)

$$
l_{c} \approx \frac{\lambda^{2}}{\Delta \lambda}
$$


mit der Zentralwellenlänge $\lambda$ sowie der spektralen Linienbreite $\Delta \lambda$ verknüpft ist, hinreichend groß sein, dass eine kohärente Überlagerung auch nach einem vollständigen Umlauf (Hin- und Rückreflexion) innerhalb der Substratschicht mit einer Brechzahl $n_{\mathrm{S}}$ und einer Dicke $h_{\text {Sub }}$ noch möglich ist (STENZEL 1996):

$$
l_{c} \geq 2 \pi \cdot n_{\mathrm{S}} \cdot h_{\mathrm{Sub}}
$$

Für Substratdicken $h_{\text {Sub }}$ von 0,7 mm (AF 45) bzw. 1,1 mm (D 263 T) und Brechzahlwerten $n_{\mathrm{S}}$ von etwa 1,5 beträgt die Mindestkohärenzlänge des Laserstrahls etwa 6,6 mm (AF 45) bzw. 10,4 mm (D 263 T).

Nach 4.16) und 4.17) kann das Auftreten von Oszillationen über die verwendete Laserlinienbreite $\Delta \lambda$ und zugehöriger Zentralwellenlänge $\lambda$ abgeschätzt werden:

$$
\Delta \lambda<\frac{\lambda^{2}}{2 \pi \cdot n_{\mathrm{S}} \cdot h_{\mathrm{Sub}}}
$$

Demnach kann bei den Proben DGWS/Nb487/40 und DGWS/Nb551/40, deren Farbstofflaser-Spektren deutliche Oszillationen zeigen (s. Abschnitt 4.2.3.1), die Linienbreite für die Wellenlänge 694,3 nm auf unter $46 \mathrm{pm}$ bestimmt werden. Für die ebenfalls mittels Farbstofflaser charakterisierte Probe DGWS/Hf331/18 ergibt sich für den Wellenlängenbereich 636,0-638,3 nm eine Laserlinienbreite von etwa 60 pm. Beide Abschätzungen unterstützen somit die vorangegangenen Betrachtungen (s. o.).

Bei der 3. Harmonischen muss die Linienbreite, um interferenzfähig zu sein, hingegen weniger als $12 \mathrm{pm}$ betragen. Mit dem zuvor bestimmten Wert von $17 \pm 2 \mathrm{pm}$ liegt dieser jedoch darüber, so dass innerhalb des Messsignals keine Oszillation auftritt (s. Abb. 4.3). Für die 4. Harmonische lässt sich die Laserlinienbreite hingegen auf 6 pm (D 263 T) bzw. etwa 10 pm (AF 45) bestimmen: Damit liegen beide Werte nahezu auf demselben Niveau der zuvor bestimmten Linienbreite von etwa $9 \pm 1$ pm. Wie die Messungen zu den Proben DGWS/Hf30/18 und DGWS/Hf30/40 in Abschnitt 4.2.1 zeigen, reichen diese Breiten für ein innerhalb des $R_{0}$-Spektrums sichtbares Interferenzsignal jedoch nicht aus. 
4.4 Diskussion und Ergebnisse

\begin{tabular}{llrrr}
\hline & \multicolumn{2}{c}{ Laserlicht- } & \multicolumn{2}{c}{ Resonanz- } \\
\cline { 2 - 5 } Probenbezeichnung & Quelle & Pol. & Wellenlänge & Winkel \\
\hline DGWS/Hf30/40 & Nd:YAG-Laser* & TE & $266,0 \mathrm{~nm}$ & $+65,0 \pm 0,05^{\circ}$ \\
DGWS/Hf30/18 & Nd:YAG-Laser* & TE & $266,0 \mathrm{~nm}$ & $+67,0 \pm 0,05^{\circ}$ \\
DGWS/Hf74/40 & Nd:YAG-Laser** & TE & $354,7 \mathrm{~nm}$ & $+46,5 \pm 0,05^{\circ}$ \\
DGWS/Hf74/40 & Nd:YAG-Laser** & TM & $354,7 \mathrm{~nm}$ & $+36,8 \pm 0,05^{\circ}$ \\
DGWS/Hf331/18 & Farbstofflaser & TM & $636,0-638,3 \mathrm{~nm}$ & $0,0 \pm 0,1^{\circ}$ \\
DGWS/Nb487/40 & Farbstofflaser & TE & $694,3 \mathrm{~nm}$ & $0,0 \pm 0,1^{\circ}$ \\
DGWS/Nb551/40 & Farbstofflaser & TM & $695,1 \mathrm{~nm}$ & $0,0 \pm 0,1^{\circ}$ \\
DGWS/Ta150/24 & Ti:Saphir-Laser & TE & $800 \mathrm{~nm}$ & $-31,5 \pm 0,1^{\circ}$ \\
DGWS/Ta150/24 & Ti:Saphir-Laser & TM & $800 \mathrm{~nm}$ & $-42,8 \pm 0,1^{\circ}$ \\
DGWS/Ta150/40 & Ti:Saphir-Laser & TE & $800 \mathrm{~nm}$ & $-33,8 \pm 0,1^{\circ}$ \\
DGWS/Ta150/40 & Ti:Saphir-Laser & TM & $800 \mathrm{~nm}$ & $-44,1 \pm 0,1^{\circ}$ \\
\hline GWS/Nb150/42 & Ti:Saphir-Laser & TE & $800 \mathrm{~nm}$ & $+42,0 \pm 0,1^{\circ}$ \\
\hline
\end{tabular}

Tab. 4.2: Experimentell bestimmte Resonanzwinkel unter Angabe der verwendeten Laserstrahlquelle, Wellenlänge und Polarisation.

Im Hinblick auf eine Zusammenfassung aller erzielten Ergebnisse gibt Tab. 4.2 einen Überblick zu den experimentell bestimmten Resonanzwinkeln der Proben unter Angabe der verwendeten Resonanzwellenlänge, Laserstrahlquelle und Polarisation. Das zur Charakterisierung der Proben verwendete Wellenlängenspektrum erstreckt sich dabei vom UV bis in den NIR-Bereich. Im Vergleich zu Tab. 4.1, welche aufgrund ungenauer Brechzahldaten nur Schätzwerte der Resonanzwinkel angibt, stimmen die experimentell bestimmten Winkel (mit Ausnahme der Proben DGWS/Hf30/40 und DGWS/Hf30/18) bis auf wenige Grad überein. Darüber hinaus wird bei den Proben DGWS/Nb487/40 und DGWS/Nb551/40, bei denen der Einfallswinkel mit $0^{\circ}$ fest vorgegeben ist, die Zielwellenlänge des Rubinlaser $694,3 \mathrm{~nm}$ am ehesten erreicht.

Zusätzlich listet Tab. 4.3 die entsprechende Reflektivität $R_{0}$ bzw. Transmittivität $T_{0}$ aller Proben auf. Neben den experimentellen Daten sind auch die theoretischen, mittels RCWA-Rechnungen vorausgesagten Werte dargestellt. Außer bei den Proben DGWS/Hf331/18 und GWS/Nb150/42 ist bei den Rechnungen die Extinktion

*4. Harmonische der Fundamentalwellenlänge 1064,14 nm.

**3. Harmonische der Fundamentalwellenlänge 1064,14 nm. 


\begin{tabular}{lrrrrrr}
\hline & & Resonanz- & \multicolumn{2}{c}{ Reflektivität $R_{0}$} & \multicolumn{2}{c}{ Transmittivität $T_{0}$} \\
\cline { 3 - 5 } Probenbezeichnung & Pol. & Wellenl. & Daten & RCWA & Daten & RCWA \\
\hline DGWS/Hf30/40 & TE & $266,0 \mathrm{~nm}$ & $87 \pm 1 \%$ & $90 \%$ & $-^{*}$ & - \\
DGWS/Hf30/18 & TE & $266,0 \mathrm{~nm}$ & $82 \pm 1 \%$ & $93 \%$ & $-^{*}$ & - \\
DGWS/Hf74/40 & TE & $354,7 \mathrm{~nm}$ & $87 \pm 1 \%$ & $94 \%$ & $<1 \pm 1 \%$ & $0 \%$ \\
DGWS/Hf74/40 & TM & $354,7 \mathrm{~nm}$ & $67 \pm 1 \%$ & $54 \%$ & $7 \pm 1 \%$ & $3 \%$ \\
DGWS/Hf331/18 & TM & $636,0 \mathrm{~nm}^{\dagger}$ & $-* *$ & - & $38 \pm 1 \%$ & $\approx 5 \%$ \\
DGWS/Nb487/40 & TE & $694,3 \mathrm{~nm}$ & $91 \pm 1 \%$ & $100 \%$ & $6 \pm 1 \%$ & $0 \%$ \\
DGWS/Nb551/40 & TM & $695,1 \mathrm{~nm}$ & $87 \pm 1 \%$ & $100 \%$ & $13 \pm 1 \%$ & $0 \%$ \\
DGWS/Ta150/24 & TE & $800 \mathrm{~nm}$ & $98 \pm 2 \%$ & $100 \%$ & $1 \pm 2 \%$ & $0 \%$ \\
DGWS/Ta150/40 & TE & $800 \mathrm{~nm}$ & $96 \pm 2 \%$ & $100 \%$ & $1 \pm 2 \%$ & $0 \%$ \\
DGWS/Ta150/40 & TM & $800 \mathrm{~nm}$ & $100 \pm 2 \%$ & $100 \%$ & $3 \pm 2 \%$ & $0 \%$ \\
\hline GWS/Nb150/42 & TE & $800 \mathrm{~nm}$ & $44 \pm 2 \%$ & $100 \%$ & $25 \pm 2 \%$ & $0 \%$ \\
\hline
\end{tabular}

Tab. 4.3: Experimentell bestimmte (Daten) sowie berechnete RCWA Reflektivitäten $R_{0}$ bzw. Transmittivität $T_{0}$ aller Proben unter Angabe der verwendeten Wellenlänge und Polarisation.

berücksichtigt worden. Vergleicht man die $T_{0^{-}}$und $R_{0^{-}}$Daten miteinander, so ist in Abhängigkeit von der verwendeten Wellenlänge deutlich eine Tendenz zu erkennen: Mit Ausnahme der Probe GWS/Nb150/42 ist mit zunehmender Anregungswellenlänge eine höhere Reflektivität bzw. niedrigere Transmittivität zu verzeichnen. Dieser Trend wird auch bei den $T_{0^{-}}$und $R_{0^{-}}$Werten der RCWA-Rechnungen beobachtet und kann auf die für die kurzen Wellenlängen ungeeignete Gitterperiode $360 \mathrm{~nm}$ zurückgeführt werden. Diese Periode erzeugt für kürzere Wellenlängen höhere Beugungsordnungen, deren Licht nicht zur Anregung der Resonanz beiträgt. Des Weiteren wird das Resonanzverhalten einiger Proben durch das Auftreten einer Woodschen Anomalie beeinträchtigt (s. Abschnitt 4.2.1 und 4.2.2), welche immer dann in Erscheinung tritt, wenn der Propagationswinkel der Wellenleitermode nahe am Abbruchkriterium für Totalreflexion liegt (s. Abschnitt 2.4.1). Durch eine gezielte Anpassung der Wellenleiterdicke an die Wellenlänge des Anregungslichts kann das Auftreten dieser Anomalie jedoch verhindert werden.

\footnotetext{
*Transmittivität ist aufgrund starker Absorption nicht detektierbar.

${ }^{* *}$ Reflektivität ist experimentell nicht erfasst worden.

${ }^{\dagger}$ Anregung bei der Wellenlänge 638,3 nm ergibt dieselbe Transmittivität.

${ }^{\dagger \dagger}$ Wert berücksichtigt experimentell bestimmte Absorption (s. Abschnitt 4.2.3.2.
} 
Aus Tab. 4.3 hervorzuheben sind indes die vollständig industriell gefertigten Proben DGWS/Ta150/24 und DGWS/Ta150/40: Für die NIR.Wellenlänge $800 \mathrm{~nm}$ beträgt die gemessene Reflektivität bis zu $100 \pm 2 \%$ bzw. die gemessene Transmittivität etwa $1 \pm 2 \%$. Damit können beide DGWStProben als möglicher Reflektor / Spiegel oder Filter für diesen Spektralbereich in Betracht gezogen werden. Darüber hinaus stellen diese Proben ihr Anwendungspotential als Sensorplattform zur Fluoreszenzanregung von Farbstoffmolekülen in Kapitel 5 unter Beweis.

Die drei innerhalb des UV-Bereichs charakterisierten Proben DGWS/Hf30/40, DGWS/Hf30/18 und DGWS/Hf74/40 erreichen hingegen eine maximale Reflektivität von $87 \%$ (94\% RCWA) und liegen somit unterhalb verwertbarer Zahlenwerte, die auf einen möglichen Einsatz als Spiegel oder Filter schließen lassen. Neben der bereits angesprochenen Gitterperiode ist vor allem die Absorption der Substratmaterialien AF 45 und D 263 T für die Resonanz als nachteilig zu sehen. Bedingt durch die Absorption werden die Proben mit abnehmender Wellenlänge ab etwa $300 \mathrm{~nm}$ zunehmend intransparent und machen eine Charakterisierung der Proben DGWS/Hf30/40 und DGWS/Hf30/18 bei $266 \mathrm{~nm}$ in Transmission sogar unmöglich. Damit erweisen sich die verwendeten SCHOTT $^{\circledR}$-Gläser AF 45 und D 263 T für einen Einsatz im UV-Wellenlängenbereich als weitgehend ungeeignet.

Dennoch, mit einer optimierten UV Materialauswahl, beispielsweise mit dem Einsatz von Fluoriden (THIELSCH 2003) als Wellenleiter- bzw. Substratmaterialien sowie die Verwendung geeigneter, speziell für den UV-Bereich angepasster Gitterperioden, kann das Transmissions- bzw. Reflexionsverhalten der DWG jedoch noch optimiert werden. Sogar eine Anwendung als VUV-Spiegel scheint - zumindest theoretisch (PEREIRA u. a. 2002) - möglich .

Ergänzend zu den bisherigen Daten sind in Tab. 4.4 für sämtliche Proben - mit Ausnahme von DGWS/Hf30/18 und DGWS/Hf30/40 - die spektrale Resonanzbandbreite bzw. Resonanzwinkelbreite unter Angabe der verwendeten Resonanzwellenlänge und Polarisation aufgelistet. Die in Klammern gesetzten Werte entsprechen dabei der Umrechnung nach Formel 2.59) in die jeweils andere Bandbzw. Winkelbreite und sollen einen Vergleich der winkelabhängigen Messung mit den spektral charakterisierten Proben ermöglichen. Eine evtl. durch die endliche Ausdehnung (ca. 0,5 mm) der $18 \mathrm{~nm}$ tiefen Gitter auftretenden Resonanzverbreite- 


\begin{tabular}{lrrrr}
\hline & & \multicolumn{3}{c}{ Resonanz- } \\
\cline { 3 - 5 } Probenbezeichnung & Polarisation & Wellenlänge & Bandbreite & Winkelbreite \\
\hline DGWS/Hf30/40 & TE & $266,0 \mathrm{~nm}$ & - & $-^{*}$ \\
DGWS/Hf30/18 & TE & $266,0 \mathrm{~nm}$ & - & $-^{*}$ \\
DGWS/Hf74/40 & TE & $354,7 \mathrm{~nm}$ & $(\approx 34 \mathrm{~nm})$ & $7,8^{\circ}$ \\
DGWS/Hf74/40 & TM & $354,7 \mathrm{~nm}$ & $(\approx 3 \mathrm{~nm})$ & $0,6^{\circ}$ \\
DGWS/Hf331/18 & TM & $636,0-638,3 \mathrm{~nm}$ & $0,3 \mathrm{~nm}$ & $\left(\approx 0,05^{\circ}\right)$ \\
DGWS/Nb487/40 & TE & $694,3 \mathrm{~nm}$ & $0,6 \mathrm{~nm}$ & $\left(\approx 0,1^{\circ}\right)$ \\
DGWS/Nb551/40 & TM & $695,1 \mathrm{~nm}$ & $0,7 \mathrm{~nm}$ & $\left(\approx 0,1^{\circ}\right)$ \\
DGWS/Ta150/24 & TE & $800 \mathrm{~nm}$ & $1,8 \mathrm{~nm}$ & $\left(\approx 0,3^{\circ}\right)$ \\
DGWS/Ta150/40 & TE & $800 \mathrm{~nm}$ & $4,6 \mathrm{~nm}$ & $\left(\approx 0,9^{\circ}\right)$ \\
DGWS/Ta150/40 & TM & $800 \mathrm{~nm}$ & $0,8 \mathrm{~nm}$ & $\left(\approx 0,2^{\circ}\right)$ \\
\hline GWS/Nb150/42 & TE & $800 \mathrm{~nm}$ & $6,5 \mathrm{~nm}$ & $\left(\approx 0,6^{\circ}\right)$ \\
\hline
\end{tabular}

Tab. 4.4: Experimentell bestimmte Resonanzbandbreite bzw. Resonanzwinkelbreite aller Proben unter Angabe der verwendeten Wellenlänge und Polarisation. Die in Klammern gesetzten Werte entsprechen der jeweils umgerechneten Resonanzbreite.

rung bei verbreitertem Strahldurchmesser (BOYE und KOstuk 2000, BENDICKSON u.a. 2001) wird aufgrund fehlender Strahlparameter vernachlässigt.

Nach Tab. 4.4 besitzen die Proben DGWS/Nb487/40 und DGWS/Nb551/40 sowie die durchstimmbare Probe DGWS/Hf331/18 eine vergleichsweise schmalbandige Resonanz von etwa $0,6 \mathrm{~nm}$ und $0,7 \mathrm{~nm}$ bzw. $0,3 \mathrm{~nm}$. Insbesondere die beiden erstgenannten Proben besitzen eine vergleichsweise dicke Wellenleiterschicht, welche unter dem gegebenen Resonanzwinkel die Anregung einer höheren Transversalmode ermöglicht und im Vergleich zur monomodigen Anregung eine höhere Schmalbandigkeit fordert (THURMAN und MORRIS 2003). Zusätzlich - wie vermutet wird - ist beim Aufbringen der Wellenleiterschicht (bedingt durch ein anisotropes Schichtwachstum) ein relativer Gitterversatz erzeugt worden [vgl. Abb. 2 bei (BRAZAS u. a. 1995)], welcher die Phasenanpassung aller interferierenden Teilstrahlen durch einen zusätzlichen Phasenversatz stört und infolgedessen die angeregte Resonanz in ihrer Breite, aber auch in ihrer Reflektivität bzw. Transmittivität (vgl. Tab. 4.3), maßgeblich zu beeinflussen scheint. Nach Überlegungen zur Optimierung des Strukturmodells, wonach eine deutlich dünnere Wellenleiterschicht,

${ }^{*}$ Wert kann experimentell nicht bestimmt werden (s. Abschnitt 4.2.1. 
eine verringerte Wellenleiterbrechzahl sowie eine evtl. Anpassung der Gitterperiode sinnvoll erscheint, ist jedoch ein Resonanzverhalten denkbar, welches im Bezug zu den Proben DGWS/Ta150/24 und DGWS/Ta150/40 ähnliche $R_{0^{-}}$bzw. $T_{0^{-}}$-Werte und zugleich eine schmalbandige Resonanzbreite aufweist.

Als einzige GWS zeigen die $R_{0^{-}}$bzw. $T_{0^{-}}$Messwerte der Probe GWS/Nb150/42 im Vergleich zu den übrigen DGWS-Proben sowohl in Reflektivität als auch in Transmittivität kein ausgeprägtes Resonanzverhalten. Neben der bereits in Abschnitt 3.4 gezeigten inhomogenen Gitterstruktur, welche zu Streuverlusten führt, ist als weitere Ursache die hohe Materialabsorption des Wellenleiters $\mathrm{Nb}_{2} \mathrm{O}_{5} \mathrm{zu}$ sehen, welcher mittels EBPVD gefertigt ist und somit nur eine geringe Packungsdichte mit vergleichsweise hoher Absorption aufweist (s. Abschnitt 3.3). Zu einem ähnlichen Schluss kommen auch andere Arbeiten, welche sich ebenfalls mit der Charakterisierung dieser GWS-Probe beschäftigt haben (BADER u. a. 2004, 2006).

Abschließend kann man feststellen: Aufgrund der spezifischen Eigenschaften der dielektrischen Wellenleitergitter, wie Schmalbandigkeit, Polarisationsabhängigkeit und einfacher Strukturaufbau, scheint die DWG für einen Einsatz in der integrierten Optik, speziell für die Anwendung als dielektrischer Spiegel oder Filter, geeignet zu sein (BADER 2006). Insbesondere die Durchstimmbarkeit von DWG Proben, welche anhand der Probe DGWS/Hf331/18 mittels Temperaturerhöhung erfolgreich gezeigt wird (s. Abschnitt 4.2.3.2), bietet zum einen die Möglichkeit, Fertigungstoleranzen bei der Herstellung der DWG zu kompensieren und zum anderen die Resonanzverschiebung für verschiedene Anwendungen (wie durchstimmbare Spiegel / Filter) gezielt auszunutzen. Im Vergleich zu bereits etablierten dielektrischen Spiegel- oder Filtersystemen zeigt diese zudem eine vergleichsweise große Durchstimmbarkeit (EBERT und EBERT 2002, LEITEL u. a. 2006). Darüber hinaus wird die DWG bereits vereinzelt als integriertes und zudem aktives Element innerhalb von Halbleiterlasern erprobt (WEBER und PAWLOWSKI 2000; PAWLOWSKI u. a. 2000). Die dabei erzielten Laserpulse zeigen eine Pulsdauer im Sub-Pikosekundenbereich und lassen sich zudem über einen Wellenlängenbereich von $5 \mathrm{~nm}$ (ohne Modensprung) durchstimmen. Des Weiteren ist mit zweidimensionalen Strukturen (2D-GWS), welche unter Resonanz keine Polarisationsabhängigkeit mehr zeigen, die Eignung als optischer Filter in der Nachrichtentechnik untersucht worden (WEBER u. a. 2004). 



\section{Wellenleitergitter als Sensorplattform}

Wie bereits in Abschnitt 2.5 gezeigt, besitzt die DWG unter Resonanzbedingungen an der Wellenleiteroberfläche ein ausgeprägtes elektromagnetisches, mit zunehmendem Abstand von der Oberfläche jedoch schnell abklingendes (evaneszentes) Feld. Motiviert durch die sog. TIRF-Mikroskopie (engl. total internal reflection fluorescence microscopy, kurz TIRFM), bei der auf der Oberfläche eines Probenträgers aufgebrachte Farbstoffe - sog. Fluorophore - über ein evaneszentes Feld zum Leuchten (Fluoreszenz) angeregt werden (AXELROD u.a. 1992), erscheint es naheliegend, die DWG - insbesondere die DGWS mit einem breiten Resonanzspektrum - als Plattform für Fluoreszenzanregung zu nutzen. Im Gegensatz zur TIRFM, bei der das evaneszente Feld durch die Totalreflexion, d.h. unter Berücksichtigung des kritischen Winkels, erzeugt wird (AXELROD u. a. 1984), ist es bei der DWG hingegen möglich, den Resonanzwinkel so zu modellieren, dass eine Anregung auch beispielsweise bei $0^{\circ}$ möglich ist (s. Abschnitt 2.5).

Zum Nachweis biologischer oder chemischer Stoffe hat darüber hinaus der Einsatz von dielektrischen Koppelgittern bei planaren Wellenleitern (TIEFENTHALER und LUKOSZ 1989) bereits zu verschiedenen Methoden geführt. So beschäftigen sich beispielsweise die etablierten Verfahren WIOS- nach der engl. Bezeichnung wavelength-interrogated optical sensors (WIKI und KUNZ 2000) - bzw. die OWLS - engl. optical waveguide lightmode spectroscopy (RAMSDEN 1997) - mit der Auswertung von ein- bzw. ausgekoppeltem Licht. Da bei der OWLS bzw. WIOS die Anregung einer Wellenleitermode zur Informationsgewinnung des zu untersuchenden Stoffs im Vordergrund steht, unterscheiden sich diese Methoden jedoch von der lokalen Modenanregung (Leckwelle) einer DWG unter Resonanzbedingungen. Mit einem vollständig gitter-strukturierten jedoch vielschichtigen Wellenleiter gelingt es Challener u. a. (2000), das unter Resonanz erzeugte evaneszente Feld in $R_{0^{-}}$ Geometrie zur Auswertung von Adsorptionseigenschaften biologischer Stoffe auf der Oberfläche auszunutzen. Mit hingegen nur einer wellenleitenden Schicht zeigt 
NEuschäFER u.a. (2003), dass mit der DGWS als Sensorplattform im Vergleich zu gängigen Glassubstraten eine Felderhöhung von bis zu zwei Größenordnungen erzielt werden kann. Die dabei bestimmte Fluoreszenzverstärkung wird mithilfe von auf der Oberfläche immobilisierten Fluorophoren erreicht, welche bei der Anregungswellenlänge 632,816 nm über Ein-Photonen-Absorptionsprozesse zur Fluoreszenz (engl. one-photon fluorescence - OPF) angeregt werden. Die Detektion des OPF-Signals erfolgt in Reflexionsgeometrie bei einem Resonanzwinkel von $0^{\circ}$ und kann sogar auf kommerzielle Großanlagen mit pharmazeutischem Anwendungsfeld ausgeweitet werden (BUDACH u. a. 2003).

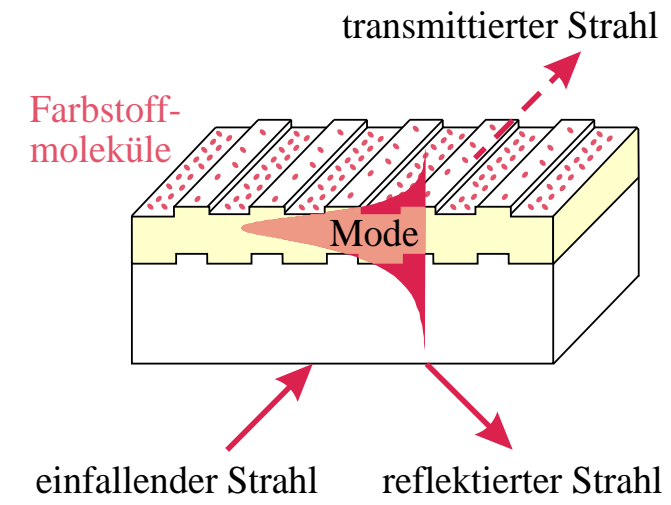

Abb. 5.1: Skizze zur Anregung von Farbstoffmolekülen auf der Oberfläche einer DGWS bei resonanter Bestrahlung über die Rückseite. Das evaneszente Feld ist hier über eine TE -Mode im Bereich der wellenleitenden Schicht (gelb) dargestellt.
Für die Detektion des Fluoreszenzsignals kann u. U. von Vorteil sein, wenn die Anregung, d. h. resonante Bestrahlung, der DWG von der Rückseite (Substratseite) erfolgt (s. u.). Zudem erfolgt die Anregung der zur Fluoreszenzanregung genutzten Probe DGWS/Ta150/40 ausschließlich bei Resonanzwinkeln von deutlich über $0^{\circ}$. Abb. 5.1 zeigt eine schematische Darstellung zur Anregung von Farbstoffmolekülen auf der Oberfläche dieser DGWS Probe. Das durch die TEMode angedeutete evaneszente Feld wird im Falle einer resonanten Anregung durch den rückseitig eingestrahl-

ten Laserstrahl erzeugt. Die exakte Feldverteilung bei resonanter Anregung der Probe DGWS/Ta150/40 ist in Abschnitt 2.5 zu sehen. Darüber hinaus kann die Anregung sowie die Detektion der Fluoreszenz durch die Trennung beider Signale verbessert werden.

Mit der Anregung von Fluorophoren durch Zwei-Photonen-Absorptionsprozesse, dessen quantenmechanischer Prozess bereits 1929 von GÖPPERT angedeutet und wenig später ausführlich beschrieben worden ist (GÖPPERT-MAYER 1931), ist es zudem möglich, das von den Farbstoffen ausgesendete Zwei-Photonen- 
Fluoreszenzlicht (engl. two-photon fluorescence, kurz TPF entfernt von der Emissionswellenlänge anzuregen (XU und WEBB 1997): Bei der TPF wird der Farbstoff im Unterschied zur OPF mit Anregungslicht doppelter Wellenlänge bestrahlt, was den Vorteil hat, dass das anregende Licht eine (effektiv) nur halb so große Photonenenergie besitzt (s. u.) und dadurch ungewollte Effekte, wie z. B. Photobleichung, vermieden werden. Eine spektrale Überlappung von Anregungswellenlänge und Emissionsspektrum wie bei der OPF tritt damit nicht mehr auf, wodurch u. a. Vorteile bei der Detektion des Fluoreszenzsignals entstehen (LAKOWICZ 2006).

Nach einer Einführung zur TPF-Anregung von Fluoreszenzfarbstoffen in Abschnitt 5.1 werden in den darauf folgenden Abschnitten die Experimente zur TPF. Anregung geeigneter Farbstoffe auf der Oberfläche der Probe DGWS/Ta150/40 beschrieben: Dazu geht Abschnitt 5.2 auf die Bestimmung der TPF Verstärkung durch DGWS bei resonanter Anregung der Struktur mittels FemtosekundenLaserpulsen der Wellenlängen $800 \mathrm{~nm}$ ein; Abschnitt 5.3 zeigt die eigentliche Anwendung der DWG als Sensorplattform am Beispiel des angeregten Peptidkomplexes mit Pikosekunden-Laserpulsen der Wellenlänge $840 \mathrm{~nm}$.

\subsection{Zwei-Photonen-Anregung von Fluoreszenzfarbstoffen}

Die Anregung von Zwei-Photonen-Fluoreszenzlicht (TPF) stellt einen nichtlinearen Absorptionsprozess dar, bei dem das Fluoreszenzsignal $I_{\mathrm{TPF}}$ quadratisch vom Anregungslicht $I$ abhängt:

$$
I_{\mathrm{TPF}} \sim I^{2}
$$

Bei dieser sog. Zwei-Photonen-Anregung (engl. two-photon excitation, kurz TPE) wird der Farbstoff über Zwei-Photonen-Absorptionsprozesse (engl. two-photon absorption - TPA angeregt. Durch ein sog. Biphoton, welches (quantenmechanisch) ein verschränktes Photonenpaar darstellt, wird bei der TPE annähernd die gleiche Energie bereitgestellt wie bei der Ein-Photonen-Anregung (engl. one-photon excitation, kurz OPE. 
Ausgehend von einer Photonenenergie $W_{\mathrm{p}}$, welche mit $W_{\mathrm{p}}=\mathrm{h} \cdot \mathrm{c}_{0} / \lambda$ umgekehrt proportional zur Wellenlänge $\lambda$ ist, lässt sich die Energie des Biphotons $W_{\text {TPE }}$ mit den Wellenlängen $\lambda_{1}$ bzw. $\lambda_{2}$ durch die folgende Gleichung beschreiben:

$$
W_{\mathrm{TPE}}=\mathrm{h} \cdot \mathrm{c}_{0} \cdot \frac{\lambda_{1}+\lambda_{2}}{\lambda_{1} \cdot \lambda_{2}}
$$

die beiden Konstanten $c_{0}$ und $\mathrm{h}$ stehen für die Vakuumlichtgeschwindigkeit sowie das PlanCKsche Wirkungsquantum.

Eine mit der Absorption von Strahlung verbundene Größe ist der Absorptionsquerschnitt $\sigma_{\overline{\text { TPA }},}$ welcher einen Wirkungsquerschnitt für die Zwei-Photonen-Absorption von Strahlung beim Durchgang von Materie beschreibt. Über die sog. Quantenausbeute oder -effizienz $\eta$, welche die Anzahl der umgesetzten Moleküle pro Anzahl absorbierter Photonen beschreibt, kann der TPE-Wirkungsquerschnitt $\sigma_{\text {एTE }}$ berechnet werden (ALBOTA u. a. 1998):

$$
\sigma_{\overline{\mathrm{TPE}}}=\eta \cdot \sigma_{\overline{\mathrm{TPA}}} \cdot
$$

Mit einem vergleichsweise hohen TPE-Wirkungsquerschnitt zählen die Xanthene, zu welchen die Fluoreszenzfarbstoffe Rhodamin B und Tetramethyl-Rhodamin (TMR) gehören, zu den gebräuchlichsten Fluorophoren (FISCHER u. a. 1995). Alle Rhodamine fluoreszieren bei Wellenlängen im sichtbaren Spektralbereich, wobei sich das Farbspektrum abhängig von der jeweiligen chemischen Struktur von GelbGrün bis Orange-Rot erstreckt. Ursprünglich für den Einsatz in Farbstofflasern bestimmt, werden Rhodamine heute insbesondere bei der Fluoreszenzmikroskopie (z. B. TIRFM) als Markerfarbstoff (Fluoreszenzlabel) eingesetzt. Das im Rahmen dieser Arbeit verwendete Rhodamin B, dessen Zentralwellenlänge im Fluoreszenzspektrum bei $610 \mathrm{~nm}$ liegt, besitzt mit etwa $70 \%$ eine vergleichsweise hohe Quanteneffizienz (KUBIN und FlETCHER 1982). Der TPE-Wirkungsquerschnitt $\sigma_{\text {TTPE }}$ von Rhodamin B liegt damit für die Anregungswellenlängen $800-840 \mathrm{~nm}$ in der Größenordnung von $10^{-48} \mathrm{~cm}^{4} \mathrm{~s} /$ Photon (XU und WeBB 1996). Aufgrund ihrer chemischen Struktur lassen sich die Rhodamine leicht in organischen Flüs- 
sigkeiten, wie Ethanol oder Methanol, lösen und können daher in verschiedenen Lösungskonzentrationen verwendet werden.

Darüber hinaus wird in Abschnitt 5.3 der Markerfarbstoff Lucifer Yellow (LY) verwendet, dessen TPE-Wirkungsquerschnitt für die Anregungswellenlänge $840 \mathrm{~nm}$ etwa $10^{-50} \mathrm{~cm}^{4} \mathrm{~s} /$ Photon beträgt und damit im Vergleich zu Rhodamin B zwei Größenordnungen kleiner ist (XU und WEBB 1996). Dieser Farbstoff wird vorwiegend aufgrund seiner nicht-toxischen Eigenschaften innerhalb von lebenden Zellen als Fluoreszenzlabel eingesetzt und besitzt zudem ein vergleichsweise breites Emissionsspektrum mit einem Maximum bei $540 \mathrm{~nm}$ (STEWART 1981).

Bei allen im Rahmen dieser Arbeit gezeigten Experimenten zur TPF. Anregung werden die Farbstoffe zunächst gelöst (z.B. in Ethanol oder Wasser) und anschließend in Tropfenform auf die Oberfläche der DGWS. Proben gebracht. Um die Fluorophore auf der Oberfläche der DGWS zu immobilisieren, wird das Lösungsmittel vollständig verdampft. Dies geschieht beim Ethanol aufgrund des niedrigen Siedepunktes relativ schnell; während

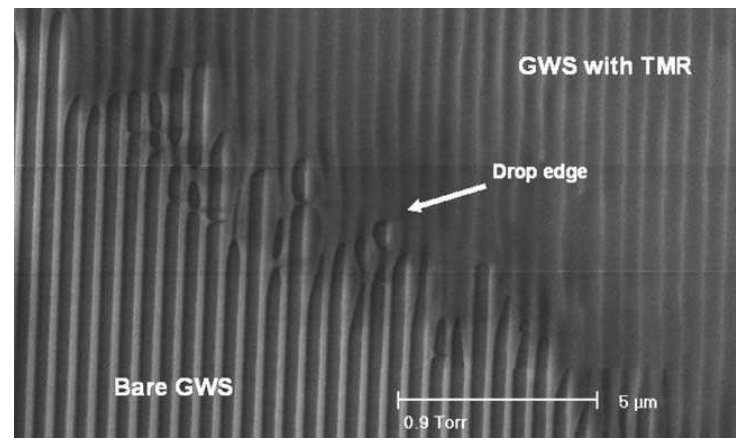

Abb. 5.2: REM-Aufnahme vom Rand eines TMR-Films auf der Oberfläche einer GWS (Quelle: Katchalski u. a. 2005b). die Verdampfung des Wassers nur bei Temperaturen deutlich über der Zimmertemperatur beschleunigt wird. Um eine Abschätzung der Molekülverteilung auf der Oberfläche nach dem Verdampfen des Lösungsmittels zu erhalten, zeigt Abb. 5.2 eine REM-Aufnahme eines eingetrockneten TMR-Tropfens auf einer GWS-Oberfläche (KATCHAlski u. a. 2005b). Das Bild zeigt in einem ca. $18 \times$ $11 \mathrm{~mm}^{2}$ großen Ausschnitt den Rand eines ca. $65 \mathrm{~nm}$ dicken TMR-Films GWS with TMR ) sowie die GWS-Oberfläche ohne Farbstoff (Bare GWS). Deutlich ist eine inhomogene Verteilung des Farbstofffilms zu erkennen, welche zum Rand (Drop edge) hin augenscheinlich eine stärkere Konzentration aufweist. Eine ähnliche Verteilung kann auch bei Rhodamin B nach der Verdampfung des Lösungsmittels Ethanol beobachtet werden. Als Ursache ist der an den Rändern höhere Verdunstungsstrom 
zu sehen, welcher innerhalb des Farbstofftropfens zu einer inhomogenen Verteilung der Kapillarkräfte führt (DEEGAN u. a. 1997).

\subsection{Zwei-Photonen-Fluoreszenzverstärkung}

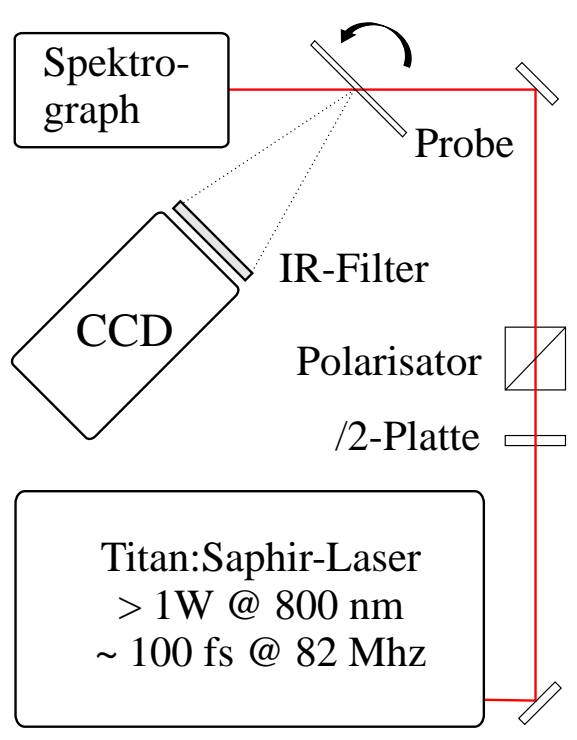

Abb. 5.3: Messaufbau mit Strahlengang zur TPFAnregung von Fluorophoren mit FemtosekundenLaserpulsen.
Die Bestimmung der TPF Verstärkung auf der Oberfläche der Probe DGWS/Ta150/40 geschieht mithilfe von FemtosekundenLaserpulsen eines Ti:Saphir-Lasers (s. Abschnitt 4.2.2). Abb. 5.3 zeigt den verwendeten Messaufbau zusammen mit dem Strahlengang des modengekoppelten Lasers. Bei einer Repetitionsrate von $82 \mathrm{MHz}$ emittierte der Laser Pulse mit einer Pulsdauer von ca. $100 \mathrm{fs}$. Mit Laserleistungen von $>1 \mathrm{~W}$ sind bei Annahme Fourierlimitierter GaUss-Pulse Spitzenleistungen von über $115 \mathrm{~kW}$ möglich; dies entspricht einer Pulsenergie von etwa 12 nJ. Das etwa 9 nm-breite Laserspektrum mit einer Zentralwellenlänge von $800 \mathrm{~nm}$ kann in seiner Schwingungsrichtung und -intensität über die Kombination von $\lambda / 2$-Platte und Polarisator festgelegt werden. Zur resonanten Bestrahlung der Probe DGWS/Ta150/40 wird die DGWS auf einem drehbaren Probentisch montiert, wodurch sich der Resonanzwinkel über die NoniUs-Skala des Drehtisches einstellen lässt. Zur Detektion des TPF-Signals wird eine gekühlte CCD-Kamera vom Typ SensiCam QE (PCO IMAGING) sowie ein Weitwinkel-Zoomobjektiv (Nikkor 18-70 mm f/3,5-4,5 G ED) der Firma NiKOn verwendet. Um den Streuanteil des Anregungslichts auf der CCD (engl. charge-coupled device) zu verringern, ist vor dem Objektiv ein Set aus drei IR-Sperrfiltern vom Typ Calflex $X^{T M}$ (s. Abb. A.3 positioniert. Nach einer Strecke im Strahlengang von rund $100 \mathrm{~cm}$ beträgt der Strahl auf der Probenoberfläche etwa 1,5 mm im Durchmesser (FWHM). 
Um einen TPF Nachweis resonant angeregter Farbstoffe zu bringen, werden verschiedene Konzentrationen von Rhodamin B in Ethanol gelöst und Konzentrationsreihen mit Konzentrationen von $5 \cdot 10^{-7} \mathrm{~mol} / \mathrm{dm}^{-3}$ bis $10^{-4} \mathrm{~mol} / \mathrm{dm}^{-3}$ angefertigt. Anschließend werden die Konzentrationen mithilfe einer EPPENDORF-Pipette in $5 \mu \mathrm{l}$ großen Tropfen auf die Probenoberfläche gebracht. Nach Verdampfen des Lösungsmittels bleibt nur ein eingetrockneter Farbstofffilm zurück. Der Durchmesser des annähernd kreisförmigen Farbstofffilms beträgt bei allen Konzentrationen im Mittel $2 \mathrm{~cm}$, so dass nach dem Trocknen der Rhodamin-B-Konzentration $10^{-4} \mathrm{~mol} / \mathrm{dm}^{-3}$ eine nahezu monolagige Molekülschicht mit einer Dichte von etwa $\sim 10^{-12} \mathrm{~mol} / \mathrm{mm}^{2}$ entsteht. Eine evtl. auftretende Eigenfluoreszenzlöschung (engl. quenching) kann somit für alle Konzentrationen vernachlässigt werden (LAKOWICZ 2006). Aufgrund des anisotropen Verdunstungsstroms bildet sich jedoch am Rand des Films eine erhöhte Farbstoffkonzentration aus (ähnlich Abb. 5.2). Da ausschließlich Fluorophore aus dem Zentrum des Films angeregt werden, muss an dieser Stelle von einer geringeren Moleküldichte als dem berechneten Mittelwert ausgegangen werden.

Aufgrund der Tatsache, dass bei der Anregung der Fluorophore unterschiedliche Bereiche derselben Probe DGWS/Ta150/40 benutzt werden, variiert der Resonanzwinkel um bis zu $0,1^{\circ}$. Dies wird darüber hinaus aufgrund der unterschiedlich starken Farbstoffkonzentrationen von einer Resonanzdrift überlagert, welche jedoch nicht dokumentiert ist. Bei der Bestrahlung der Probe durch die Zentralwellenlänge $800 \mathrm{~nm}$ wird somit ein mittlerer Resonanzwinkel von ca. $-34^{\circ}$ für die TE-Polarisation und etwa $-44^{\circ}$ (vgl. Abschnitt 4.2.2) für die TM-Polarisation bestimmt. Aufgrund der großflächigen Gitterstruktur der DGWS sowie der Anregung der Farbstoffmoleküle mit Femtosekunden-Laserpulsen ist es zudem nicht notwendig, den Laserstrahl zu fokussieren. Damit kann zunächst aus der Pulsenergie von $12 \mathrm{~nJ}$ sowie der Photonenenergie von etwa $1,55 \mathrm{eV}$ die Photonenanzahl von rund $49 \cdot 10^{9}$ pro Puls bestimmt werden. Aus dem Strahldurchmesser sowie der zeitlichen Pulslänge (FWHM kann eine Photonenstrom-

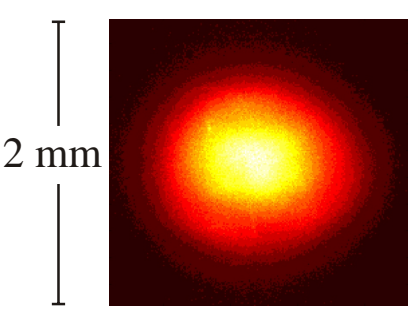

Abb. 5.4: CCD-Kamerabild einer TPFAnregung auf einer DGWS-Oberfläche. dichte von rund $2 \cdot 10^{25}$ Photonen $/\left(\mathrm{cm}^{2} \cdot \mathrm{s}\right)$ abgeleitet 
werden. Aufgrund dieser vergleichsweise geringen Photonenstromdichte (FISCHER u. a. 1995) sowie der Reproduzierbarkeit der TPF-Experimente können Effekte wie Photobleichung (engl. photobleaching) ausgeschlossen werden. Abb. 5.4 zeigt ein CCD-Kamerabild der TPF-Anregung einer auf der DGWS-Oberfläche getrockneten Rhodamin-B-Lösung als farbcodiertes Graustufenbild. Das Bild gibt dabei nahezu die komplette Querschnittsfläche des Laserstrahls (Strahlprofil) wieder.

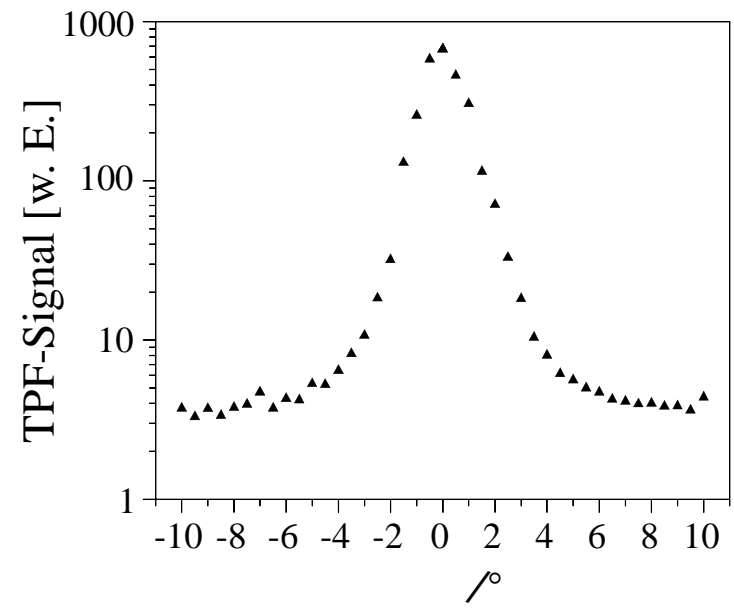

Abb. 5.5: Winkelabhängiges TPF Signal mittels Ti:Saphir-Laserpulsen angeregter Rhodamin-B-Moleküle auf der Oberfläche der Probe DGWS/Ta150/40.

Bedingt durch die enge Beziehung von Resonanzwellenlänge und -winkel (Resonanzkennlinie) verschiebt sich mit der Änderung des Einfallswinkels auch die TPF-Anregungswellenlänge. Abb. 5.5 zeigt das gemessene TPF Signal von Rhodamin-B-Molekülen auf der Oberfläche der DGWSłProbe DGWS/Ta150/40 über der Änderung des Einfallswinkels $(\Delta \theta)$ in logarithmischer Darstellung. Die ursprünglich in Ethanol gelöste Konzentration von $10^{-6} \mathrm{~mol} / \mathrm{dm}^{-3}$ entspricht nach der beschriebenen mittleren Verteilung auf der Oberfläche einer Farbstoffdichte von $\sim 10^{-10} \mathrm{~mol} / \mathrm{mm}^{2}$. Wie in der Abb. 5.5 erkennbar ist, ändert sich mit Variation des Einfallswinkels das TPF. Signal um bis zu zwei Größenordnungen, wobei die Winkelakzeptanz für die Anregung nur wenige Grad beträgt. Der in der Abbildung gezeigte Verlauf des TPF Signals korreliert dabei mit dem der TPF-Anregung zugrunde liegenden Laserspektrum des einfallenden Laserstrahls (s. Abschnitt 4.2.2). Geht man bei der Änderung des Resonanzwinkels von einer gleichbleibenden Resonanzbandbreite aus, so handelt es sich um eine Überlagerung der quadratischen Anregungsintensität [vgl. (5.1)] mit dem wellenlängenabhängigen (dispersiven) TPE-Wirkungsquerschnitt von Rhodamin B. Aufgrund der begrenzten Bandbreite der DGWS muss zudem bei der Intensität des Anregungsspektrums zwischen einem resonanten bzw. nicht-resonanten Anteil unterschieden werden (s. u.). 
Um die TPF-Verstärkung der Probe DGWS/Ta150/40 zu bestimmen, erfolgt die Anregung der Fluorophore auf der DGWS Oberfläche sowohl bei resonanter als auch bei nicht-resonanter Bestrahlung. Ohne Änderung des Resonanzwinkels erfolgt die nicht-resonante Bestrahlung durch die Drehung der Polarisationsrichtung um 90, was einer Bestrahlung der Probe mit TM-polarisiertem Licht entspricht. Bei einem Winkel von ca. $34^{\circ}$ beträgt die Resonanzwellenlänge für diese Polarisation etwa $754 \mathrm{~nm}$ und liegt damit hinreichend entfernt von der Zentralwellenlänge des Anregungslichts. Während der Bestrahlung der Probe wird das von der CCD-Kamera eingefangene Licht als Bildinformation (14-Bit-Graustufenbild) gespeichert und anschließend am Computer ausgewertet.

Abb. 5.6 zeigt das integrierte, d. h. über alle Pixel summierte, CCD-Signal des fluoreszierenden Farbstoffs Rhoda$\min B$ bei verschiedenen Laserleistungen $(0-1,1 \mathrm{~W})$ bei resonanter (TE) sowie bei nicht-resonanter (TM) Anregung auf der Oberfläche der Probe DGWS/Ta150/40. Die ursprünglich in Ethanol gelöste Farbstoffkonzentration von $10^{-6} \mathrm{~mol} / \mathrm{dm}^{-3}$ entspricht unter Berücksichtigung einer anisotropen Verdunstung des Fünf-MikroliterTropfens (s. o.) einer Moleküldichte von weniger als einem Molekül auf einer Fläche von $1 \cdot 10^{-10} \mathrm{~mm}^{2}$. Mit einer Einzelmolekülfläche von ungefähr $1 \mathrm{~nm}^{2}$ (DREXHAGE 1990) und einer Anzahl von etwa $3 \cdot 10^{12}$ Molekülen pro Tropfenfläche entspricht dies einer Verteilungsdichte von weniger als $1 \%$. Die in Abb. 5.6 gezeigten Messdaten können mithilfe einer Fitfunktion $\mathrm{y}(\mathrm{x})$ nach der Methode der kleinsten
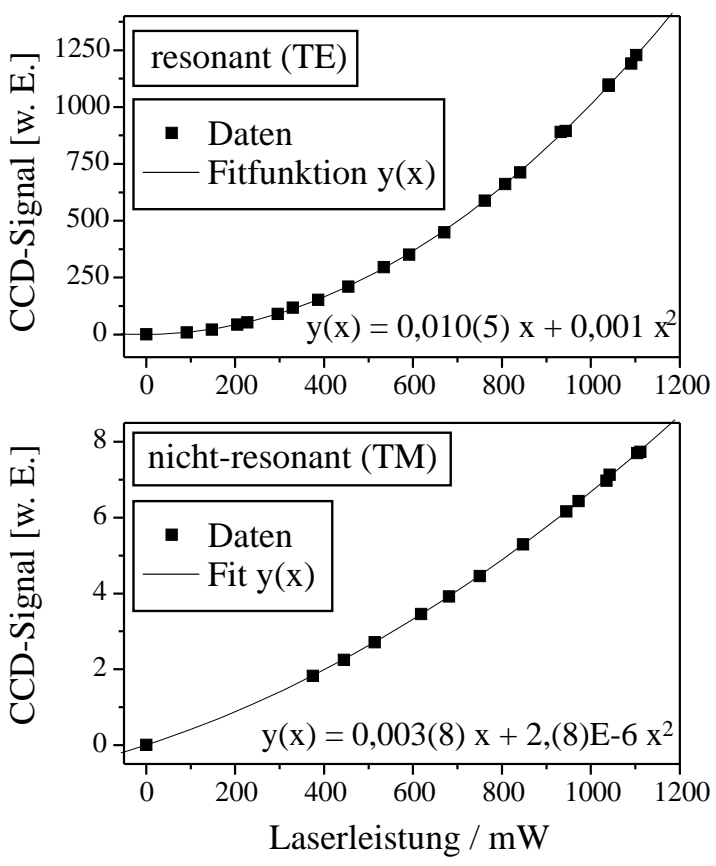

Abb. 5.6: CCD-Signal resonant (oben) bzw. nicht-resonant angeregter Rhodamin-B-Moleküle der Konzentration $\sim 10^{-12} \mathrm{~mol} / \mathrm{cm}^{2}$ auf der Oberfläche der Probe DGWS/Ta150/40 für verschiedene Laserleistungen. Quadrate angepasst werden. Dabei re- 
präsentiert der lineare Anteil dieser Annäherung das Streu- oder Hintergrundlicht, welches direkt proportional zur einfallenden Laserstrahlung ist. Dieser Anteil ist - wie erwartet - bei beiden Messungen (resonant und nicht-resonant) mit $10^{-2}$ bis $10^{-3}$ etwa gleich groß. Der zweite, nichtlineare Anteil der Fitfunktion kann durch dessen eindeutig quadratischen Verlauf der TPF-Anregung zugeordnet werden. Dieser ist bei nicht-resonanter Anregung mit einer Größenordnung von $10^{-6} \mathrm{zu}$ dem um drei Größenordnungen kleiner als bei resonanter Anregung mit $10^{-3}$.

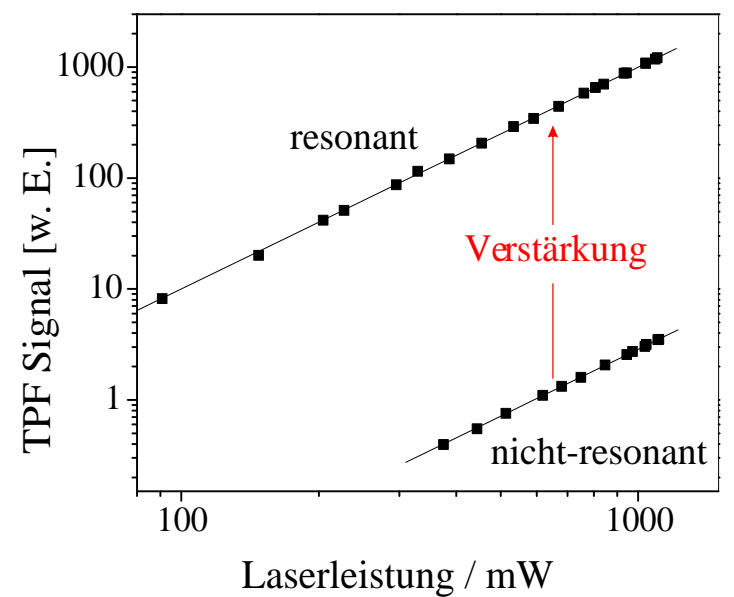

Abb. 5.7: Logarithmische Darstellung des Fluoreszenzlichts (TPF-Signal) aus Abb. 5.6 zur Bestimmung der TPF Verstärkung.

Durch den Abzug des linearen Anteils aus dem CCD,Signal erhält man die TPF-Signale, welche zur Bestimmung der TPF Verstärkung wichtig sind. Abb. 5.7 zeigt dazu die logarithmische Darstellung des TPF.Signals bei Anregung von Rhodamin B der Oberflächenkonzentration $10^{-10} \mathrm{~mol} / \mathrm{mm}^{2}$ für beide Anregungsformen. Über die zusätzlich dargestellten Fitkurven (Steigung 2) kann der TPF-Verstärkungsfaktor direkt bestimmt werden und liegt für diese Konzentration bei 350. Dieser Wert stellt den maximal gemessenen TPF Verstärkungsfaktor dar KAPPEL u. a. 2004c). Bei unterschiedlichen Konzentrationen und wiederholten Messungen kann nach Auswertung der Messdaten stets ein dreistelliger Verstärkungsfaktor mit einem mittleren Wert von $276 \pm 65$ bestimmt werden. Die von KAPPEL u. a. (2005) gezeigten Verstärkungswerte mit zunehmender Rhodamin-B-Konzentration suggerieren indes eine Abhängigkeit des Verstärkungsfaktors von der verwendeten Konzentration. Dies muss jedoch aufgrund einer Standardabweichung von \pm 65 aller experimentell bestimmten Werte kritisch betrachtet werden. Darüber hinaus zeigt die Arbeit von FRICKE-BEGEMANn u. a. (2005), dass auch ein TPF-Nachweis bei vergleichsweise großflächiger Anregung der DGWS möglich ist und zudem mithilfe eines sog. diffraktiven optischen Elements (DOE) eine TPF-Anregungsmatrix auf der Probenoberfläche erzeugt werden kann (MAROWSKY u.a. 2004). 


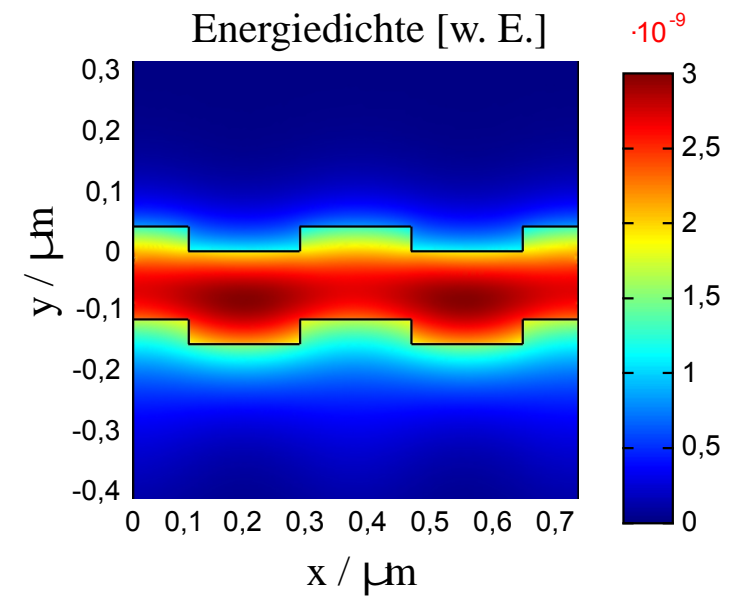

(a) TE-Anregung (resonant).

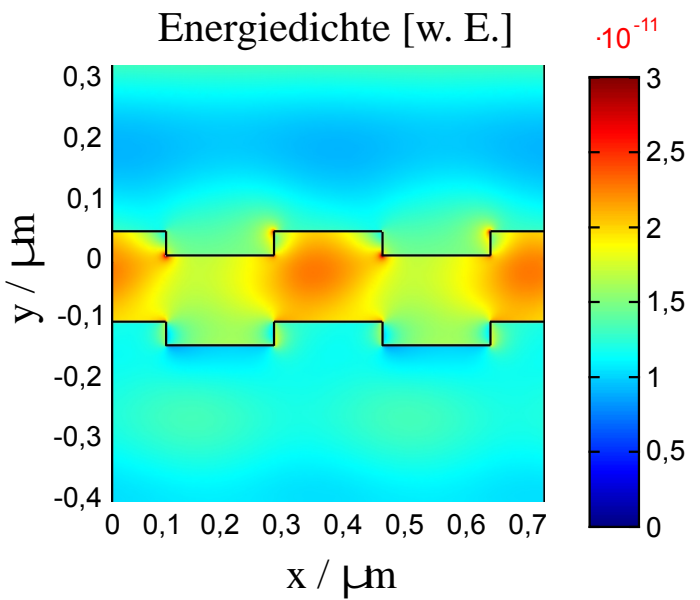

(b) TM-Anregung (nicht-resonant).

Abb. 5.8: FEM-Simulationen zur Energiedichte bei Bestrahlung der Probe DGWS/Ta150/40 unter (a) Resonanzbedingungen (TE) bzw. (b) bei nicht-resonanter Anregung durch die um $90^{\circ}$ gedrehte Polarisationsrichtung (TM), jedoch bei gleicher Anregungswellenlänge $800 \mathrm{~nm}$ und festem Einfallswinkel von $-31,82^{\circ}$.

Bei der TPF Anregung von TMR auf derselben DGWS-Oberfläche kann im Vergleich zu einem BK7-Glassubstrat für ein ca. $8 \mathrm{~nm}$ breites Anregungsspektrum bei der Zentralwellenlänge $815 \mathrm{~nm}$ und einem Einfallswinkel von $36^{\circ}$ ein ähnlich großer Wert von 330 bestimmt werden (SORIA u. a. 2005, THAYIL u. a. 2005).

Bei einer GWS-Probe mit einer Gitterperiode von $523 \mathrm{~nm}$ wird hingegen für die Anregungswellenlänge $844 \mathrm{~nm}$ im Vergleich zur TIRFAAnregung ein TPF Verstärkungsfaktor von 3 (SORIA u. a. 2004a) bestimmt und im Vergleich zur nichtresonanten TM-Anregung ein Faktor von 10 (KATCHALSKI u. a.|2005b) nachgewiesen. Als Ursache für den um eine Größenordnung geringeren TPFFFaktor sind die vergleichsweise großen Strukturabmessungen (Wellenleiterdicke $430 \mathrm{~nm}$ bzw. Gittertiefe $450 \mathrm{~nm}$ ) zu sehen, welche eine effiziente Anregung der Fluorophore durch das schnell abklingende (evaneszente) Feld der Leckwelle verhindern.

Zusätzlich zu den experimentellen Ergebnissen lässt sich der Verstärkungsfaktor der Probe DGWS/Ta150/40 anhand von FEM-Simulationen theoretisch berechnen. Dazu ist in Abb. 5.8 die Verteilung der Energiedichte bei (a) resonanter bzw. (b) nicht-resonanter Bestrahlung der DGWS gleicher Intensität zu betrachten. Die 
dargestellten Simulationsflächen $\left(0,7 \times 0,7 \mu^{2}\right)$ zeigen in der Mitte die gitterstrukturierte Wellenleiterschicht der DGWS sowie die angrenzenden Medien Luft (oberhalb) und D 263 T-Substrat (unterhalb). Detaillierte Informationen zu den verwendeten Strukturkonstanten und Randbedingungen können dem Abschnitt 2.5 entnommen werden. Der für die Anregungswellenlänge $800 \mathrm{~nm}$ berechnete TE Resonanzwinkel bei maximaler Energiedichte im Wellenleiter beträgt dabei 31,82 . Beim Vergleich der farbcodierten Werteskalen in den Abbildungen 5.8a bzw. 5.8b ist ein deutlicher Unterschied in den Energiedichten zu erkennen. Die Skalen sind dabei so angepasst, dass der Unterschied in den Maxima (rot) genau zwei Größenordnungen ausmacht. Bei der genaueren Betrachtung von Abb. 5.8b fällt zudem auf, dass es an den Kanten des Gitterprofils zu Erhöhungen der Energiedichte kommt. Der Grund dafür ist in der Geometrie des Gitterprofils zu sehen, welche durch ihre spitz zulaufende Form eine Antennenwirkung zeigt und es daher zur Anregung eines lokalen elektrischen Feldes (Nahfeld) kommt. Dieser Effekt wird u. a. bei der optischen Rasternahfeldmikroskopie (engl. scanning nearfield optical microscope, kurz SNOM ausgenutzt, um die Auflösungsgrenze des klassischen Lichtmikroskops zu unterschreiten (NOvOTNY und HECHT 2006).

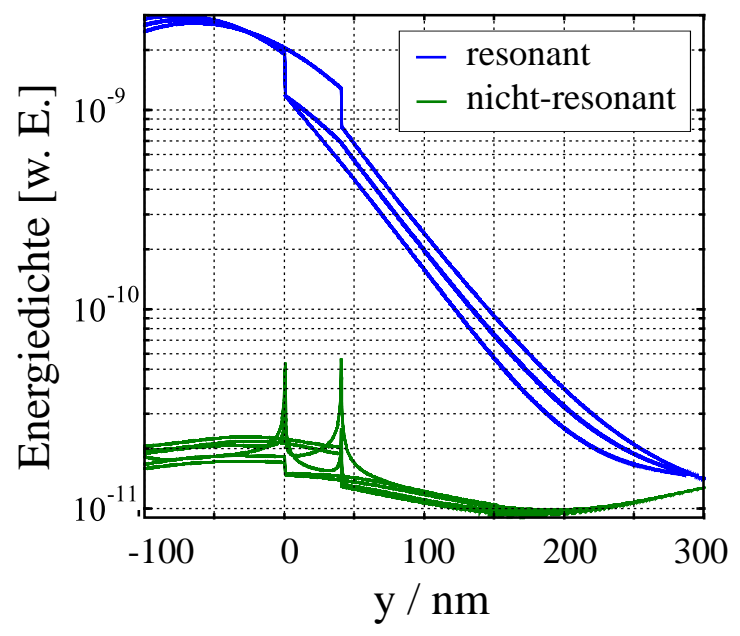

Abb. 5.9: Zwölf vertikale Schnittlinien entlang der DGWS-Oberfläche zu Abb. 5.8 zeigen die Energiedichteverteilung bei resonanter bzw. nicht-resonanter Anregung.
Um die Unterschiede der Energieverteilung beider Anregungsformen zu verdeutlichen, können entlang der DGWS. Oberfläche Schnittlinien gelegt werden: Danach zeigt Abb. 5.9 für die TE bzw. TM-Anregung die Verteilung der Energiedichte mit zunehmendem Abstand von der DGWS-Oberfläche für zwölf vertikale, gleichverteilte Schnittlinien zu Abb. 5.8a bzw. 5.8b, Dabei markieren die gedachten Linien bei $y=$ $0 \mathrm{~nm}$ bzw. $y=40 \mathrm{~nm}$ den Übergang von der Gitterstruktur des wellenleitenden Mediums zum Medium Luft; die negativen y-Werte zeigen die Energiedichteverteilung innerhalb des Wellenleiters. 
Wie man in Abb. 5.9 sieht, beträgt der Unterschied in der Energiedichte zwischen resonanter und nicht-resonanter Anregung an den Medienübergängen etwa zwei Größenordnungen. Darüber hinaus ist an den Übergängen $y=0 \mathrm{~nm}$ bzw. $40 \mathrm{~nm}$ für beide Anregungsformen ein Sprung (Unstetigkeit) in Form eines plötzlichen Abfalls der Energiedichte zu erkennen. Weiterhin fällt auf, dass bereits nach etwa $300 \mathrm{~nm}$ die Energiedichte der resonanten Anregung auf das Niveau der nicht-resonanten Anregung bzw. auf das Niveau des Anregungsfeldes $\left(\approx 1 \cdot 10^{-11}\right.$ w. E. $)$ abgefallen ist. Da einige der Schnittlinien auch die Eckpunkte des Gitterprofils schneiden, sind bei nicht-resonanter (TM) Anregung auftretende lokale Felderhöhungen zu sehen, welche innerhalb der Kurven in Form von Spitzen erkennbar sind.

Um einen Faktor zwischen den Energiedichten bei resonanter bzw. nicht-resonanter Anregung zu bestimmen, wird die Energie direkt an der DGWS-Oberfläche (s. Abb. 5.8a bzw. 5.8b) integriert (Linienintegral). Der darüber bestimmte (lineare) Faktor mit einem Wert von rund 76 beinhaltet jedoch noch keine TPF. Anregung, welche nach (5.1) quadratisch von der Intensität bzw. Energie abhängt. Unter Berücksichtigung dieser nichtlinearen Anregungsform läge der TPF. Verstärkungsfaktor demnach mit rund 5776 im deutlich vierstelligen Bereich; allerdings würde dabei weder die gepulste Anregung noch das spektrale Resonanzverhalten der Probe DGWS/Ta150/40 berücksichtigt werden. Nach Abschnitt 4.2 .2 bzw. 4.3 besitzt die DGWS-Probe jedoch eine begrenzte Resonanzbandbreite, wodurch etwa $55 \%$ des Laserspektrums für eine resonante Anregung der Probe genutzt werden. Die TPF-Anregung der Fluorophore auf der DGWS-Oberfläche erfolgt aber nicht nur über den resonant gekoppelten Teil der einfallenden Welle, sondern wird auch vom nicht-koppelnden FRESNEL-Anteil, d.h. den restlichen $45 \%$ des Laserspektrums, ermöglicht (s. u.). Da das TPF-Signal $I_{\mathrm{TPF}}$ reziprok von der zeitlichen Pulslänge $\Delta t$ abhängt (XU und WEBB 1996):

$$
I_{\mathrm{TPF}} \sim \Delta t^{-1}
$$

müssen die in Abschnitt 4.3 bestimmten Pulslängen $\Delta t_{\mathrm{R}}=298 \pm 15$ fs (resonante Leckwelle) und $\Delta t_{\mathrm{F}}=88 \pm 4$ fs (FRESNEL-Anteil) sowie die Pulsdauer des freien Laserstrahls $\Delta t_{\mathrm{NR}}=120 \pm 6$ fs (nicht-resonante Anregung) bei der Berechnung des TPF Verstärkungsfaktors berücksichtigt werden. 
Unter der Annahme, dass die Intensität des Fresnel-Anteils $I_{\mathrm{F}}$ ebenso wie die Intensität der resonanten Leckwelle $I_{\mathrm{R}}$ einen Beitrag bei der TPF Anregung liefert, lässt sich im Vergleich zur nicht-resonanten Anregung der Intensität $I_{\mathrm{NR}}$ ein TPF. Verstärkungsfaktor

$$
V \overline{\mathrm{TPF}}=\left(\frac{I_{\mathrm{R}}}{\sqrt{\Delta t_{\mathrm{R}}}}+\frac{I_{\mathrm{F}}}{\sqrt{\Delta t_{\mathrm{F}}}}\right)^{2} \cdot\left(\frac{I_{\mathrm{NR}}}{\sqrt{\Delta t_{\mathrm{NR}}}}\right)^{-2} .
$$

Unter der Bedingung, dass bei beiden Anregungsformen dieselbe Gesamtintensität verwendet wird, also $I_{\mathrm{R}}+I_{\mathrm{F}}=I_{\mathrm{NR}}$, lässt sich mit der Substitution von $I_{\mathrm{F}}$ die Formel (5.5) umstellen:

$$
\frac{I_{\mathrm{R}}}{I_{\mathrm{NR}}}=\left(\sqrt{\frac{l_{\mathrm{TPF}}}{\Delta t_{\mathrm{NR}}}}-\frac{1}{\sqrt{\Delta t_{\mathrm{F}}}}\right) \cdot\left(\frac{1}{\sqrt{\Delta t_{\mathrm{R}}}}-\frac{1}{\sqrt{\Delta t_{\mathrm{F}}}}\right)^{-1} .
$$

Mit der aus der FEM-Simulation bestimmten Relation $I_{\mathrm{R}}=76 \cdot I_{\mathrm{NR}}$ wäre nach 5.6 im Idealfall ein TPF Verstärkungsfaktor zu erwarten gewesen, welcher mit einem Wert von 1738 bereits eine Größenordnung über dem experimentell bestimmten Wert liegt. Aufgrund von Oberflächenrauigkeiten, welche innerhalb der FEM-Simulationen nicht berücksichtigt werden, kann die Energiedichte an der DGWS-Oberfläche bei nicht-resonanter Anregung jedoch höher liegen und damit die berechnete Verstärkung abschwächen.

Mithilfe der Formel (5.6) lässt sich aus dem experimentell bestimmten (maximalen) TPF Verstärkungsfaktor $350 \pm 65$ der lineare Verstärkungsfaktor berechnen. Mit einem Wert von rund $33 \pm 7$ liegt dieser im Rahmen der Messgenauigkeit (5\% Pulslängenfehler unter Berücksichtigung der Fehlerfortpflanzung) gleichauf mit dem von NEUSCHÄFER u. a. (2003) bestimmten OPF-Verstärkungsfaktor von 39 bei Anregung einer nahezu identischen Probe mit TE-polarisiertem Licht. Einer Abschätzung nach 4.10 entspricht der in derselben Arbeit mittels TM-Anregung erzielte OPFF-Faktor von 170 aufgrund der vergleichsweise schmalen TM-Bandbreite der DGWS von 0,8 nm ( $\widehat{=} 1177 \mathrm{fs}$ ) einer TPF-Anregung mit einem zweistelligen TPF Verstärkungsniveau. Entsprechende Experimente zur Bestimmung der TPF 
Verstärkung bei Bestrahlung der Probe DGWS/Ta150/40 mittels TM-polarisierten Lichts werden im Rahmen dieser Arbeit jedoch nicht durchgeführt.

\subsection{Nachweis eines Nonapeptids in wässriger Umgebung}

Motiviert durch die zuvor erzielte TPF-Verstärkung soll nun das Anwendungspotential resonanter DGWS untersucht werden. Wie in Abschnitt 5.2 wird dazu ein Ti:Saphir-Lasersystem verwendet, welches von der Firma SPECTRA PHYsics stammt und aus einem Nd : YVO4-Pumplaser Millennia Vs sowie einer Ti:SaphirLasereinheit Tsunami besteht. Bei gleicher Repetitionsrate von $82 \mathrm{MHz}$ liefert dieser Laser jedoch im Unterschied zu dem bisher verwendeten Ti:Saphir-Laser Pikosekunden-Laserpulse mit einer Pulsdauer von etwa 1,5 ps im modengekoppelten Betrieb.

Abb. 5.10 zeigt die zur Anregung und Detektion der TPF verwendete Messanordnung mit dem Strahlengang des Ti:Saphir-Lasers. Sowohl die Polarisation als auch die Intensität der einfallenden Laserstrahlung werden wie bei den Experimenten zuvor durch die Kombination aus $\lambda / 2$-Platte und Polarisator bestimmt. Zur Detektion des Fluoreszenzlichts wird ana$\log$ zu Abschnitt 5.2 eine CCD-Kamera vom Typ SensiCam QE der Firma PCO IMAgInG sowie ein Weitwinkel-Zoomobjektiv der Firma NIKON inkl. der IR-Bandkantenfilter eingesetzt. Zur Bestrahlung der Probe im Resonanzwinkel wird selbige wieder in einen geeigneten Halter auf einem Drehtisch mit NoniUs-Skala montiert. Zur räumlichen Begrenzung des Laserstrahls auf ca. $2 \mathrm{~mm}$ (FWHM wird eine Blende verwendet, welche in geringem Abstand zur

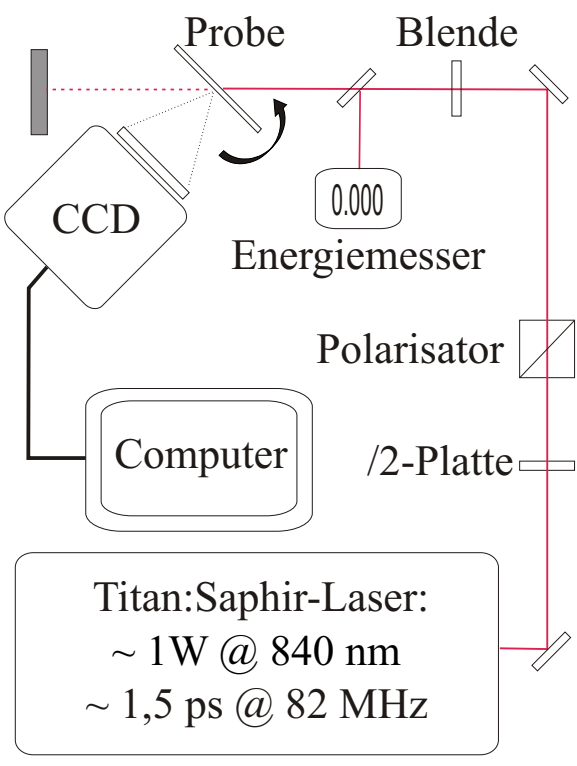

Abb. 5.10: Messaufbau mit Strahlengang zur TPF-Anregung von Fluorophoren mit PikosekundenLaserpulsen.

Probe positioniert ist. 
Mit einer maximalen Laserleistung von $1 \mathrm{~W}$ liegt die Pulsenergie - unter Annahme FourIER-limitierter Gauss-Pulse - mit 12 nJ zwar auf demselben Niveau wie bei den TPF Experimenten mittels Femtosekundenpulsen, jedoch können mit diesen Pulslängen lediglich Spitzenleistungen von $7,64 \mathrm{~kW}$ erreicht werden, die um den Faktor 15 kleiner sind als bei der Anregung mit Femtosekundenpulsen. Wie sich im Laufe der Untersuchung herausstellte, ist ohne eine Fokussierung des Laserstrahls ein TPF Nachweis der Farbstoffe bei nicht-resonanter Bestrahlung der Probe DGWS/Ta150/40 nicht möglich. Im Vergleich zu den ca. $100 \mathrm{fs}$ langen Laserpulsen (s. Abschnitt 4.2.2 bzw. 5.2 mit einer spektralen Breite von etwa $9 \mathrm{~nm}$ besitzen die Pikosekundenpulse hingegen eine Breite (FWHM $)$ von etwa $0,7 \mathrm{~nm}$. Damit reicht die etwa 4,6 nm breite TE-Resonanz der Probe DGWS/Ta150/40 aus, die Bandbreite des Lasers vollständig ein- und wieder auszukoppeln. Mit einer Zentralwellenlänge des Laserspektrums von $840 \mathrm{~nm}$ beträgt der Resonanzwinkel für TE-polarisiertes Laserlicht rund $-44^{\circ}$.

Bevor der eigentliche TPF Nachweis biologischer Stoffe auf der Oberfläche der DGWS erbracht wird, soll zunächst die TPF-Anregung des Markerfarbstoffs $\mathrm{LY}$ in den Konzentrationen $\sim 1 \cdot 10^{-4} \mathrm{~mol} / \mathrm{dm}^{-3}\left(\widehat{=} 10^{-12} \mathrm{~mol} / \mathrm{mm}^{2}\right)$ bis $\sim$ $10^{-10} \mathrm{~mol} / \mathrm{dm}^{-3}\left(\widehat{=} 10^{-6} \mathrm{~mol} / \mathrm{mm}^{2}\right)$ bei diesen Pulslängen geprüft werden. Dazu wird der Farbstoff in Ethanol gelöst und anschließend als Tropfen zu je $5 \mu \mathrm{l}$ auf die Oberfläche der DGWS gebracht. Nach vollständigem Verdampfen des Lösungsmittels sind die LY-Farbstoffmoleküle auf der Oberfläche der Probe vollständig immobilisiert. Der Durchmesser des getrockneten LYFFarbstofffilms beträgt ungefähr 15-20 mm. Durch den Farbstofffilm auf der Oberseite des Gitters ändert sich die Brechzahl in diesem Medium und verschiebt somit den Resonanzwinkel minimal (nicht dokumentiert), was jedoch mithilfe des in Abb. 5.10 angedeuteten Rotationstisches kompensiert wird. Die von der CCD-Kamera aufgenommenen monochromatischen Bilder (14-Bit-Graustufen) werden anschließend am Computer ausgewertet: Dazu werden alle relevanten Pixel des Kamerabildes integriert und der entsprechenden Anregungsleistung des Laserstrahls zugeordnet.

Abb. 5.11 zeigt das CCD-Signal bei der Anregung von LY der gelösten (bzw. immobilisierten) Konzentration (a) $10^{-4} \mathrm{~mol} / \mathrm{dm}^{-3}\left(\widehat{=} 8 \cdot 10^{-13} \mathrm{~mol} / \mathrm{mm}^{2}\right)$ bzw. (b) $10^{-9} \mathrm{~mol} / \mathrm{dm}^{-3}\left(\widehat{=} 8 \cdot 10^{-8} \mathrm{~mol} / \mathrm{mm}^{2}\right)$ bei verschiedenen Laserleistungen. Die $\mathrm{zu}-$ sätzlich dargestellten Fehlerbalken geben die Standardabweichung der jeweiligen 


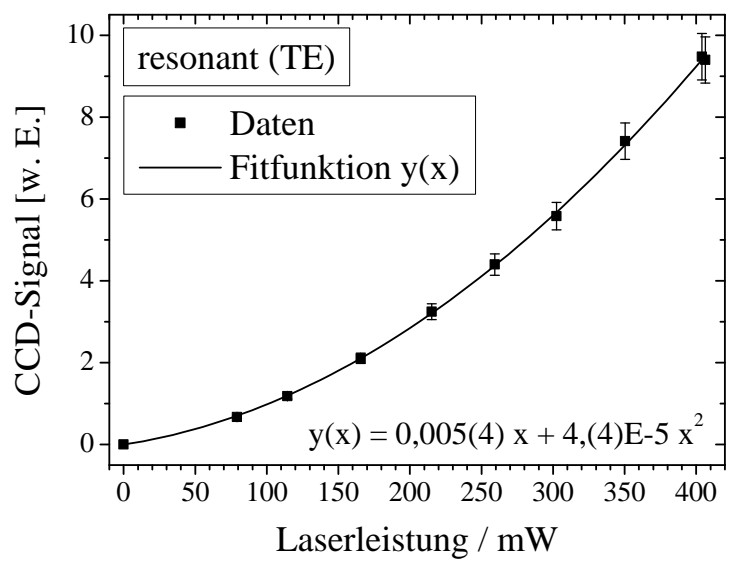

(a) LY-Konzentration $10^{-4} \mathrm{~mol} / \mathrm{dm}^{-3}$.

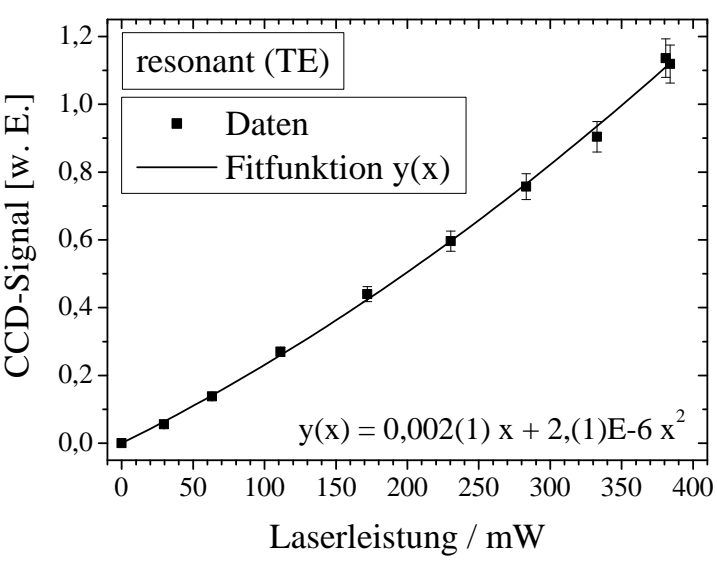

(b) LY-Konzentration $10^{-9} \mathrm{~mol} / \mathrm{dm}^{-3}$.

Abb. 5.11: CCD Signal angeregter LY-Farbstoffmoleküle auf der Oberfläche der Probe DGWS/Ta150/40 in den (gelösten) Konzentrationen (a) $10^{-4} \mathrm{~mol} / \mathrm{dm}^{-3} \mathrm{bzw}$. (b) $10^{-9} \mathrm{~mol} / \mathrm{dm}^{-3}$ bei verschiedenen Laserleistungen.

Messpunkte wieder. Photobleichung, wie sie u. a. bei Experimenten mit starker Laserstrahl-Fokussierung beobachtet wird (SÁNCHEZ u.a. 1997), kann aufgrund der Reproduzierbarkeit der TPF Anregung durch den unfokussierten Laserstrahl bei maximaler Laserintensität von ca. $400 \mathrm{~mW}$ ausgeschlossen werden. Mithilfe der in den Abbildungen dargestellten Fitfunktion $\mathrm{y}(\mathrm{x})$ zweiter Ordnung lassen sich die Messdaten in einen linearen sowie einen quadratischen Anteil zerlegen. Danach wird der nichtlineare Anteil dem TPF-Signal zugeordnet und der lineare Anteil wird auf das Oberflächenstreulicht bzw. Hintergrundlicht zurückgeführt. Während in Abb. 5.11a ein TPF-Signal noch deutlich zu erkennen ist, lässt die Fitfunktion mit einem um eine Größenordnung kleineren Anteil bereits die Nachweisgrenze von LY mit dieser Methode erkennen. Unter der Annahme eines LY Farbstofffilmdurchmessers von 18,6 mm (Mittelwert) kann für die Konzentration $8 \cdot 10^{-8} \mathrm{~mol} / \mathrm{mm}^{2}$ von einer Moleküldichte von ungefähr einem Molekül pro

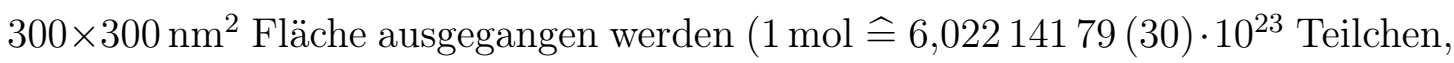
Avogadro-Konstante). Zum Vergleich: Mit dem Farbstoff Rhodamin B ist noch ein Nachweis bei $1 \cdot 10^{-10} \mathrm{~mol} / \mathrm{dm}^{-3}$ möglich (Daten nicht gezeigt), wohingegen eine eindeutige Bestimmung der TPF für die LY.Konzentration $1 \cdot 10^{-10} \mathrm{~mol} / \mathrm{dm}^{-3}$ unter Berücksichtigung der Messgenauigkeit nicht mehr möglich ist. Aufgrund des etwa 155-fach geringeren TPE-Wirkungsquerschnitts von LY gegenüber Rhoda- 
min B (XU und WEBB 1996) ist damit die TPF-Nachweisgrenze von LY mit dieser Detektionsmethode erreicht.

Nach Abschluss der Untersuchungen zum TPF-Nachweis von LY werden weitergehende Experimente mit markierten Molekülkomplexen auf der Basis resonanter DGWS in nativer Umgebung durchgeführt. Zu diesem Zweck wird zunächst der LY.Markerfarbstoff biochemisch an ein körpereigenes Peptid-Derivat gekoppelt und anschließend als Molekülkomplex zum Fluoreszenznachweis auf die DGWS-Oberfläche gebracht. Das aus neun Aminosäuren bestehende Peptid (Nonapeptid) wird komplett synthetisch hergestellt und steht stellvertretend für ein körpereigenes Leukozytenantigen (engl. human leukocyte antigen) mit der Bezeichnung HLA-B aus dem Haupthistokompatibilitätskomplex (MHC), welches die immunologischen Vorgänge im menschlichen Körper reguliert (PÖHLMANN u.a. 2004). Das mit LY markierte Nonapeptid (Derivat) lässt sich als Aminosäuresequenz der Form Arginin-Arginin-Leucin-Prolin-Isoleucin-Cystein(-LY)-Serin-ArgininLeucin beschreiben.

Für den Nachweis des Molekülkomplexes in wässriger (nativer) Umgebung bei Zimmertemperatur wird eine eigens angefertigte Küvette, welche die Probe DGWS/Ta150/40 samt Nonapeptid aufnehmen kann, verwendet. Abb. 5.12 zeigt eine Skizze der mit Wasser $\left(\mathrm{H}_{2} \mathrm{O}\right)$ gefüllten Küvette, die anstelle des Probenhalters

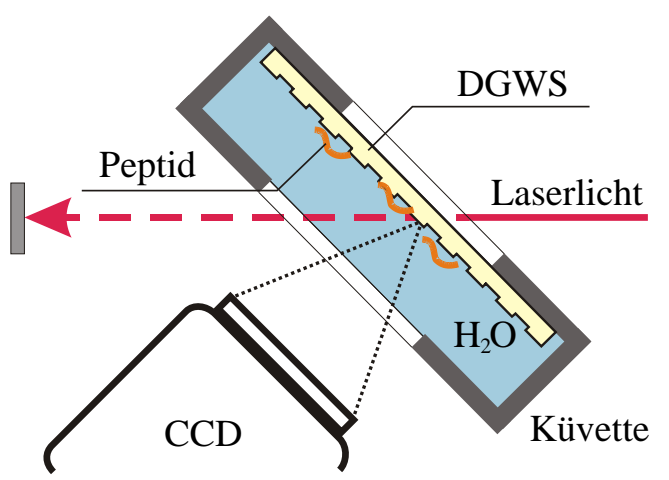

Abb. 5.12: Skizze der mit Wasser $\left(\mathrm{H}_{2} \mathrm{O}\right)$ gefüllten Küvette, welche zur TPF-Anregung des [Y] markierten Nonapeptids auf der DGWS-Oberfläche verwendet wird. im Versuchsaufbau nach Abb. 5.10 eingesetzt wurde. Der TE-polarisierte einfallende Laserstrahl trifft nun die Probe nicht mehr direkt, sondern muss erst ein transparentes Eintrittsfenster der Küvette passieren, was aufgrund der FRESNEL-Reflexion die effektiv eingestrahlte Laserleistung auf der Probe mindert. Wie zuvor koppelt der (nicht fokussierte) Laserstrahl dabei rückseitig in die DGWS.Probe ein. Die Probe selbst befindet sich dabei direkt hinter dem Eintrittsfenster und ist durch eine Klemmvorrichtung fest arretiert. 
Das Aufbringen des Molekülkomplexes auf die DGWS-Oberfläche erfolgt wie bei den Experimenten zuvor in Tropfenform $(5 \mu \mathrm{l})$. Durch einen Trocknungsprozess im Ofen bei $40^{\circ} \mathrm{C}$ wird der Molekülkomplex auf der Gitteroberfläche immobilisiert, so dass sich dieser auch in wässriger Umgebung nicht mehr oder zumindest erst nach langer Zeit (im Vergleich zur Messung) wieder löst.

Abb. 5.13 zeigt das CCD.Signal des mit Laserlicht angeregten Molekülkomplexes in der ursprünglich in $\mathrm{H}_{2} \mathrm{O}$ gelösten Konzentration $10^{-5} \mathrm{~mol} / \mathrm{dm}^{-3}$ (人 $8 \cdot 10^{-12} \mathrm{~mol} / \mathrm{mm}^{2}$ ) bei verschiedenen Leistungen. Die Messdaten werden wie zuvor mithilfe einer nichtlinearen Ausgleichsrechnung $\mathrm{y}(\mathrm{x})$ zweiter Ordnung angepasst. Deutlich ist der quadratische Anteil, welcher dem TPF-Signal entspricht, gegenüber dem linearen Signal zu erkennen. Aufgrund kleinster im Wasser gelöster Partikel schwankt das detektierte CCD-Signal bei kleinen Laserleistungen. Dies hat zur Fol-

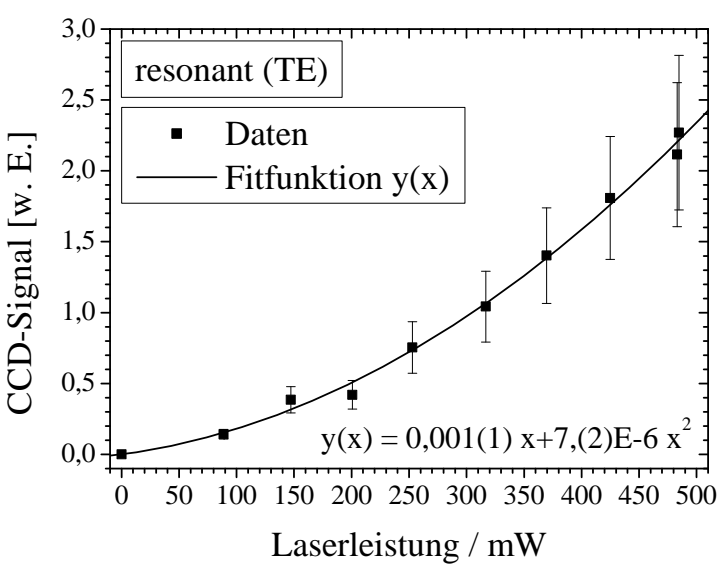

Abb. 5.13: CCD-Signal angeregter LY. markierter Nonapeptide in der Konzentration $8 \cdot 10^{-12} \mathrm{~mol} / \mathrm{mm}^{2}$ auf der Oberfläche der innerhalb der Küvette arretierten DGWS bei verschiedenen Laserleistungen. ge, dass die Standardabweichung (Fehlerbalken) des Messsignals im Vergleich zu den vorherigen Messungen größer ist. Dennoch, mit dem Nachweis eines TPF Signals eines körpereigenes Peptid-Derivats unter nativen Umgebungsbedingungen unterstreicht die DGWS ihr Anwendungspotential in der Biosensorik (SELLE u. a. 2005). Weiterhin kann das TPF-Signal eines LY markierten HLA-B-Komplexes in trockener Form (ohne Küvette) bis zu einer Konzentration von $10^{-7} \mathrm{~mol} / \mathrm{dm}^{-3}\left(\widehat{=} 8 \cdot 10^{-10} \mathrm{~mol} / \mathrm{mm}^{2}\right)$ auf der Oberfläche der Probe DGWS/Ta150/40 nachgewiesen werden (Daten nicht gezeigt). 



\section{Zusammenfassung und Ausblick}

Wie die optische Charakterisierung der im Rahmen dieser Arbeit verwendeten Proben unter Resonanzbedingungen zeigt, erreichen die gefertigten dielektrischen Wellenleitergitter mit einem vergleichsweise einfachen Strukturaufbau - bestehend aus einem Substrat sowie einer wellenleitenden Schicht mit einer inhärent periodischen Struktur - insbesondere für den nah-infraroten Spektralbereich eine Reflektivität von nahezu $100 \%$ sowie einen nahezu vollständigen Intensitätseinbruch in Transmission. Die im ultravioletten Spektralbereich gemessenen Reflektivitäten liegen sowohl für die Wellenlänge $355 \mathrm{~nm}$ als auch für $266 \mathrm{~nm}$ bei Werten um $87 \%$ und haben somit das angestrebte Ziel von $98 \%$ verfehlt. Mit einer bereits in Abschnitt 4.4 vorgeschlagenen Lösung, welche die Anpassung der verwendeten Gitterperiode zur Unterdrückung höherer Beugungsordnungen sowie den Einsatz von Fluorid-Verbindungen als Wellenleiter- bzw. Substratmaterialien zur Vermeidung stattfindender Absorption vorsieht, erscheint eine Zunahme (Abnahme) der Reflektivität (Transmittivität) jedoch denkbar. Durch die vergleichsweise hohe Wellenleiterdicke von über $500 \mathrm{~nm}$ der im sichtbaren Spektralbereich charakterisierten Proben wird zwar die erhoffte geringe Resonanzbandbreite von wenigen ÅGSTRÖM erreicht, diese führt jedoch aufgrund eines anisotropen Schichtwachstums - so die Vermutung - innerhalb der verwendeten Doppel-Gitter-WellenleiterStrukturen zu einem ungewollten, relativen Gitterversatz, welcher die Resonanz nachhaltig beeinträchtigt und zu einem weniger ausgeprägten Resonanzverhalten in Transmission bzw. Reflexion führt. Insgesamt haben die Ergebnisse der optischen Charakterisierung jedoch gezeigt, dass die dielektrischen Wellenleitergitter in der hier gezeigten Strukturqualität grundsätzlich als schmalbandiger Filter für den sichtbaren bzw. nah-infraroten Spektralbereich geeignet sind.

Neben den kommerziell erhältlichen, vorstrukturierten Substratgittern fester Gitterperiode wird die Strukturierung einer beschichteten Probe mithilfe der $\mathrm{F}_{2^{-}}$ Laserablation erprobt. Insgesamt ist jedoch die Modulationstiefe des mit dieser 
Methode erzeugten sinusförmigen Reliefgitters über die gesamte Gitterfläche zu inhomogen, als dass das Gitter bei großflächiger Bestrahlung im Hinblick auf den Interferenzcharakter der Resonanz eine hinreichend anisotrope Diffraktivität aufweisen kann. Neben den durch die Inhomogenität hervorgerufenen Streuverlusten an der lithographisch erzeugten Gitterstruktur wird das Resonanzverhalten der Struktur vor allem durch die hohe intrinsische Absorption des verwendeten $\mathrm{Nb}_{2} \mathrm{O}_{5^{-}}$ Materials beeinflusst, so dass in der Summe lediglich eine Reflektivität von etwa $44 \%$ bzw. eine Transmittivität von rund $25 \%$ erreicht wird. Zudem muss für eine großflächige Anwendung der mittels $\mathrm{F}_{2}$-Laserablation erzeugten Strukturen neben einer Optimierung des Ablationsprozesses und der Verwendung absorptionsarmer Materialien auch eine geeignete Methode zur Vergrößerung der Gitterfläche (hier $200 \times 200 \mathrm{\mu m}^{2}$ ) gefunden werden .

Bis auf wenige Einschränkungen lässt sich das Resonanzverhalten der Wellenleitergitter, insbesondere die Abhängigkeit von Resonanzwinkel und -wellenlänge in Form der sog. Resonanzkennlinie, sehr gut durch analytische Methoden beschreiben und eignet sich daher besonders für eine Modellierung resonanter Wellenleitergitter im Vorfeld von theoretischen Betrachtungen durch rigorose Rechenmethoden. Da jedoch - wie im Verlauf der Arbeit festgestellt wird - aufgrund von Fertigungstoleranzen keine hinreichende Genauigkeit der Strukturparameter erreicht werden kann, d.h. eine möglichst exakte Übereinstimmung von Modell und gefertigter DWG nicht möglich ist, erscheint es notwendig, einen zusätzlichen Freiheitsgrad innerhalb der Resonanzbedingungen zu erzeugen. Mithilfe eines spezifisch gefertigten, porösen Wellenleiters gelingt es erstmalig, in Form eines temperaturabhängigen Brechungsindexes einen veränderbaren Parameter innerhalb der resonanten Struktur zu erzeugen, wodurch im Experiment unter festem Einfallswinkel bei einer Erhöhung der Umgebungstemperatur um $14 \mathrm{~K}$ eine Resonanzverschiebung von $-2,3 \mathrm{~nm}$ erzeugt wird. Im Vergleich zu den Proben mit einem rein passiven Strukturverhalten ist mit dieser Durchstimmbarkeit auch nach Fertigung der Struktur ein Eingriff in das Resonanzverhalten in Grenzen möglich.

Im Hinblick auf die Untersuchungen der unter Resonanzbedingungen auftretenden Felderhöhung werden auf der Oberfläche der Wellenleitergitter biotechnologisch relevante Farbstoffe immobilisiert und mithilfe eines Ultrakurzpuls-Lasers durch Zwei-Photonen-Absorptionsprozesse zur Emission von Fluoreszenzlicht angeregt. 
Gegenüber nicht-resonanter Anregung wird dabei experimentell ein Verstärkungsfaktor der Fluoreszenz von deutlich über zwei Größenordnungen mit einem Maximum von 350 ermittelt. Durch die quantitative Bestimmung der zeitlichen Pulslänge der dissipativen Leckwelle mithilfe eines Autokorrelators lässt sich dieser Wert auf einen Verstärkungsfaktor bei Ein-Photonen-Fluoreszenzanregung umrechnen und mit bereits veröffentlichten Werten aus der Literatur vergleichen. Die hier mithilfe einer lichtempfindlichen Kamera vorgestellte Detektionsmethode erlaubt zudem bei resonanter Zwei-Photonen-Fluoreszenzanregung des Markerfarbstoffs Lucifer Yellow noch den Nachweis von einem Molekül pro $300 \times 300 \mathrm{~nm}^{2}$ Fläche. Am Schluss der Untersuchungen gelingt es, ein mit Lucifer Yellow markiertes körperverwandtes Peptid des menschlichen Immunsystems auf der Oberfläche einer Doppel-Gitter-Wellenleiter-Struktur in nativer, d. h. in wässriger, Umgebung nachzuweisen. Dies unterstreicht das Anwendungspotential der dielektrischen Wellenleitergitter als Sensorplattform für die Biochemie bzw. Biosensorik.

So werden sich auch zukünftige Einsatzgebiete dielektrischer Wellenleitergitter an biotechnologischen Interessenschwerpunkten orientieren. Wie der Forschungsbericht von SORIA u. a. (2004b) zeigt, können auch mit resonanten Strukturen auf der Basis eines Polymerwellenleiters bereits erfolgreich biochemische Bindungsexperimente durchgeführt werden: Durch die biochemische Affinität von Streptavidin ein Biotin-Molekül zu binden, lässt sich beispielsweise der Bindungsprozess beider Stoffe auf der Oberfläche der Wellenleitergitter verfolgen. Zudem lassen sich vereinzelte biochemische Prozesse bei Zellen verfolgen: Wie die Arbeit von FANG u.a. (2006) zeigt, können mit den nur wenige $100 \mathrm{~nm}$ ausgedehnten evaneszenten Feldern die intrazellulären Aktivitäten innerhalb einer wenige Mikrometer großen lebenden Zelle beobachtet werden. Damit stellen die Wellenleitergitter ihre Möglichkeiten als Fluoreszenz-Sensorplattform im Hinblick auf biochemische bzw. molekularbiologische Anwendungen schon heute unter Beweis. Bezüglich einer kommerziellen Verwendung dieser Strukturen sollte zuvor das seit 2001 von der Novartis AG gehaltene Patent zur Anwendung der Wellenleitergitter als Sensorplattform (NOVACHIP ${ }^{\circledR}$ ) in Augenschein genommen werden (BUDACH und NEUSCHAEFER ).

Darüber hinaus zeigen verschiedene Patente zur Anwendung von Wellenleitergittern in der Sensorik (WAWRO u. a. 2007, 2008) sowie Arbeiten zur Anwendung als 
Filter in der Telekommunikation (NIEDERER 2004; NIEDERER u. a. 2004), dass eine Abstimmung der Resonanzwellenlänge auch ohne einen zusätzlichen Freiheitsgrad innerhalb der Resonanzkennlinie durch die Neigung des Resonanzwinkels, welcher mithilfe einer hochpräzisen, elektromechanischen Miniaturplattform justiert wird, möglich ist. Mit den Untersuchungen von BLOCK (2005) scheint sogar ein Einsatz der Strukturen als frequenzselektiver Reflektor innerhalb eines externen Resonators frei-emittierender Laserdioden (engl. External-cavity diode lasers) denkbar. Unter Berücksichtigung auftretender Kreuzungspunkte polarisationsabhängiger Resonanzkennlinien (vgl. TE,TM-Schnittpunkt bei etwa $610 \mathrm{~nm}$ in Abb. 4.1a) kann unter festem Einfallswinkel eine polarisationsunabhängige Anregung der Wellenleitergitter erfolgen (LACOUR u. a. 2001, 2003). Mit dem Einfall von zirkular polarisiertem Licht, welches als Superposition einer transversal-elektrischen und einer transversal-magnetischen Welle verstanden wird, lässt sich der Schnittpunkt der simultanen TE-TM-Anregung spektral erweitern, wodurch eine polarisationsunabhängige Durchstimmbarkeit der Wellenleitergitter erreicht werden kann (NIEDERER u. a. 2005, GRINVALD u. a. 2008. 


\section{A Anhang}

\section{Optische Eigenschaften verwendeter Gläser und Filter}

Im Nachfolgenden ist das Transmissionsverhalten zweier Substratgläser sowie einem optischen Sperrfilter dargestellt. Es handelt sich dabei um Herstellerangaben.

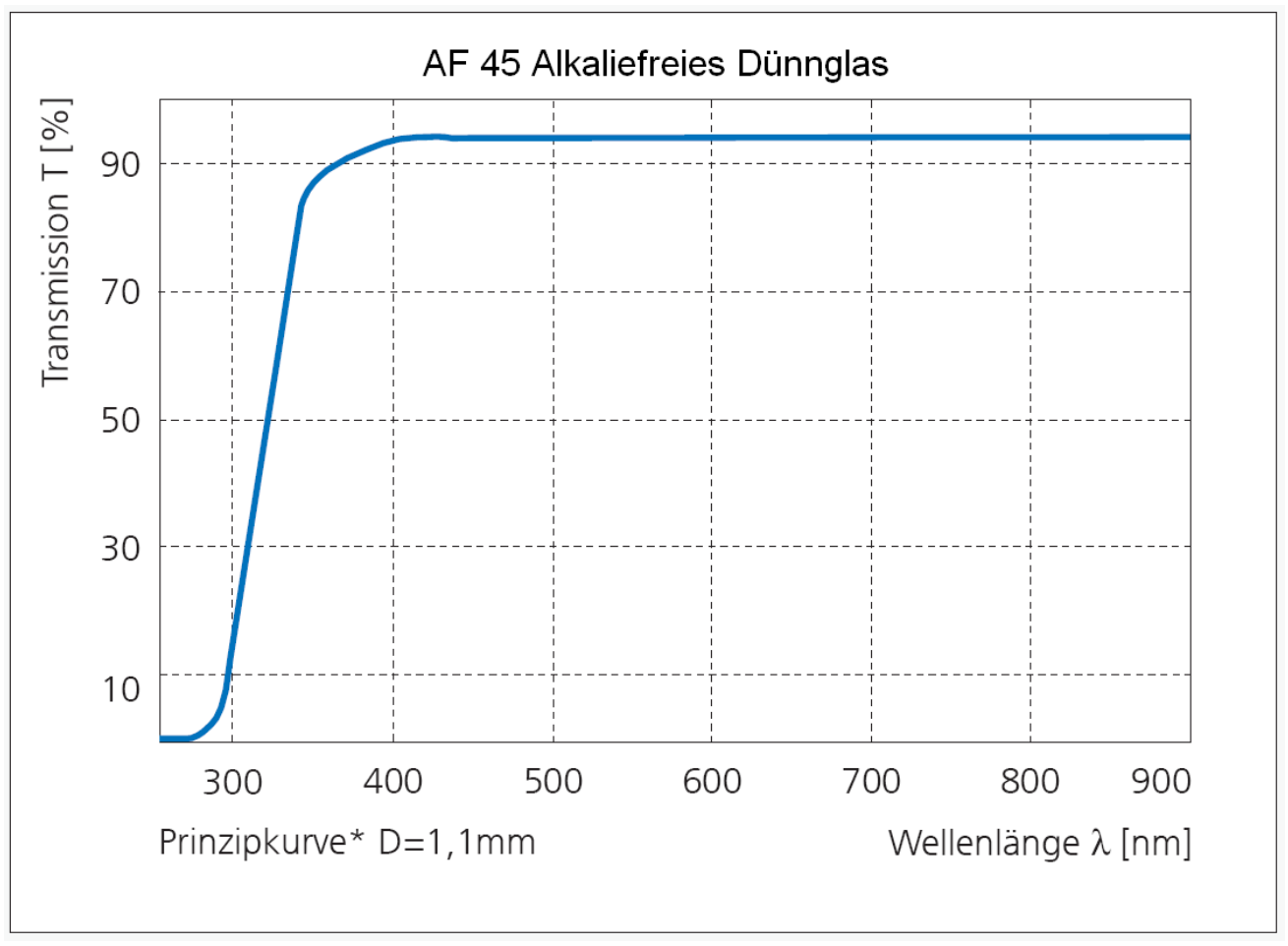

Abb. A.1: Herstellerangaben zum Schотт ${ }^{\circledR}$ _Glas AF 45. 


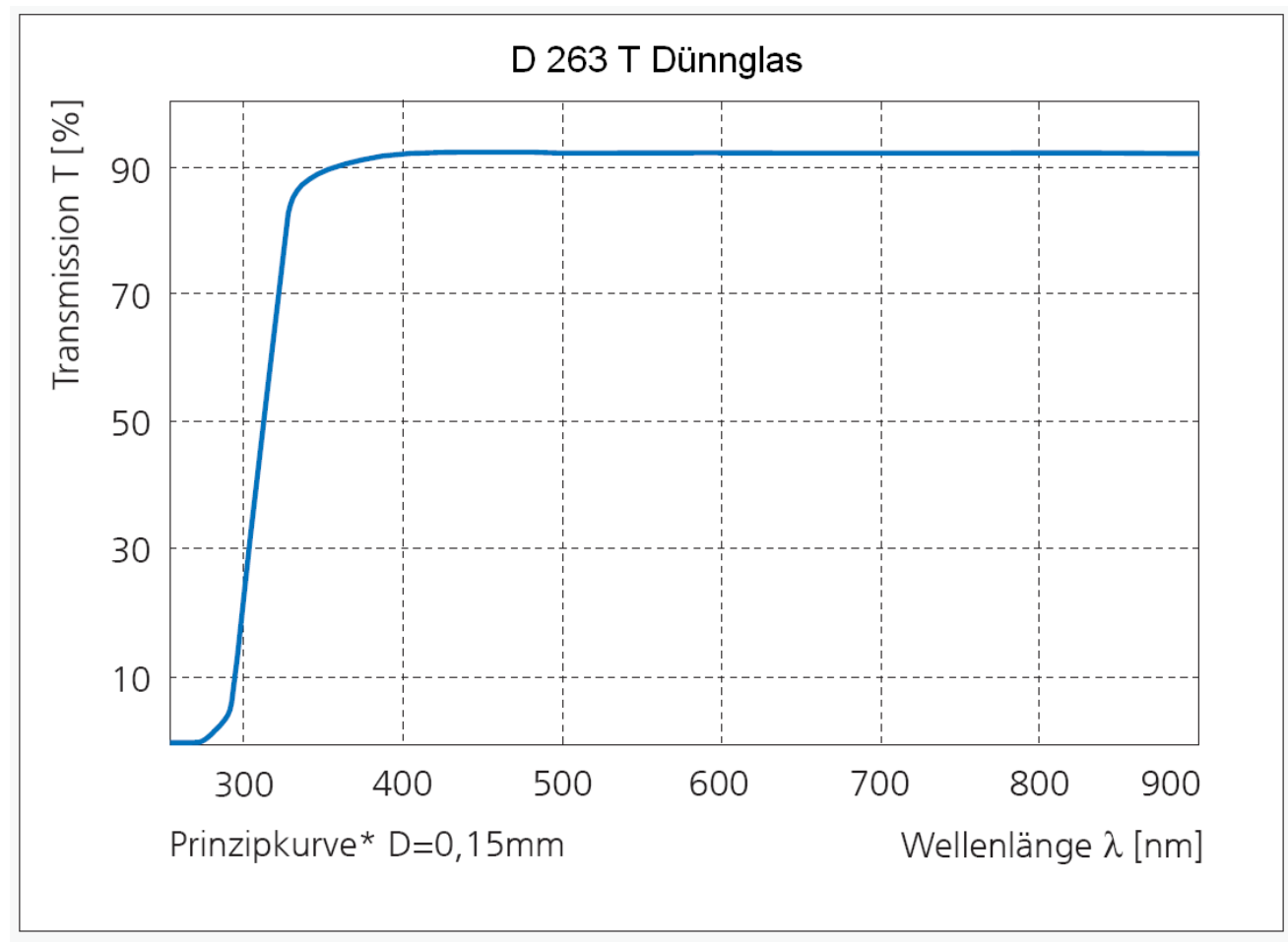

Abb. A.2: Herstellerangaben zum Sснотт ${ }^{\circledR}$ _Glas D $263 \mathrm{~T}$.

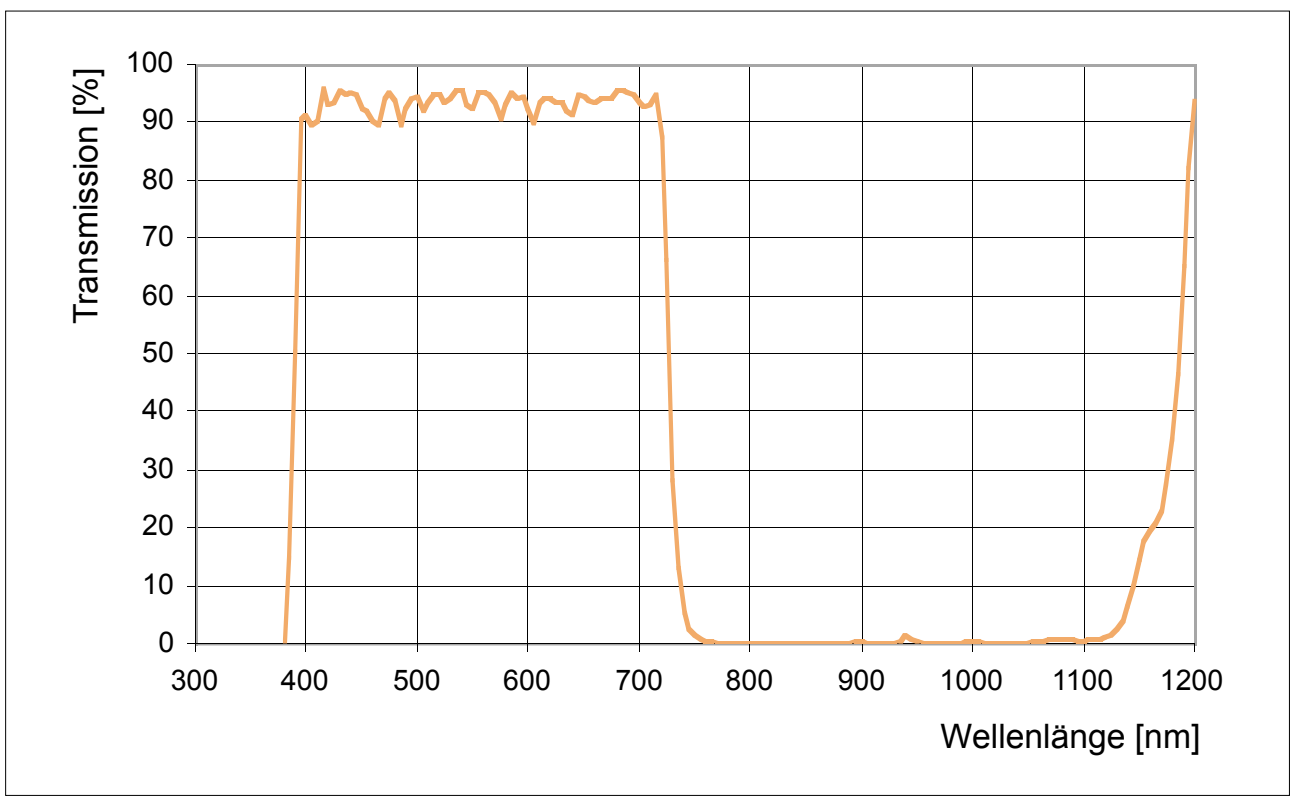

Abb. A.3: Herstellerangaben zum UnAXIs-Filter Calflex $X^{T M}$. 


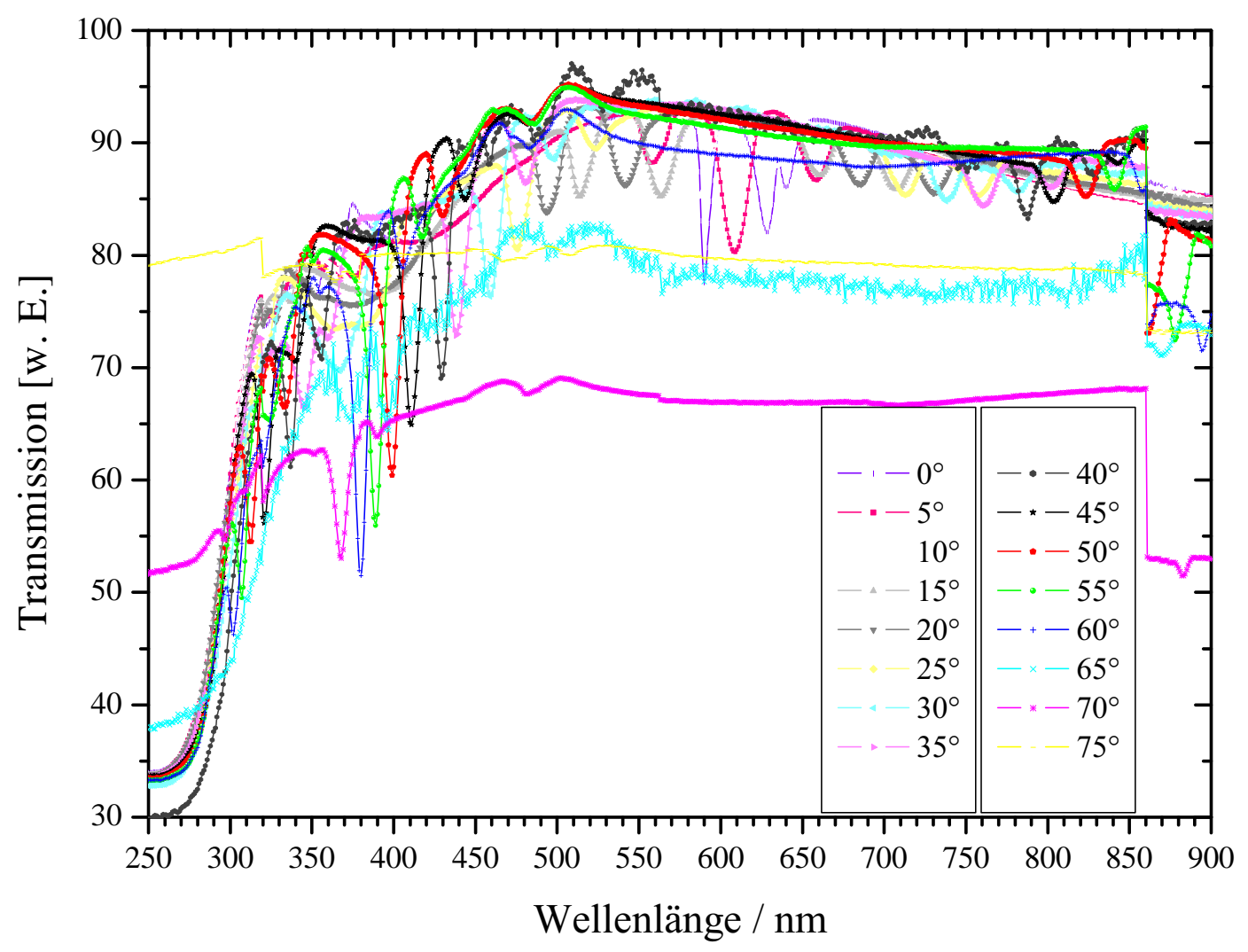

Abb. A.4: 16 Spektrometer-Datensätze der Probe DGWS/Ta150/40 für verschiedene Einfallswinkel $\left(0^{\circ}-75^{\circ}\right)$ zur Bestimmung der TE, bzw. TM-Resonanzkennlinie (s. Abschnitt 4.1). 


\section{Schematischer Aufbau des verwendeten Autokorrelators}

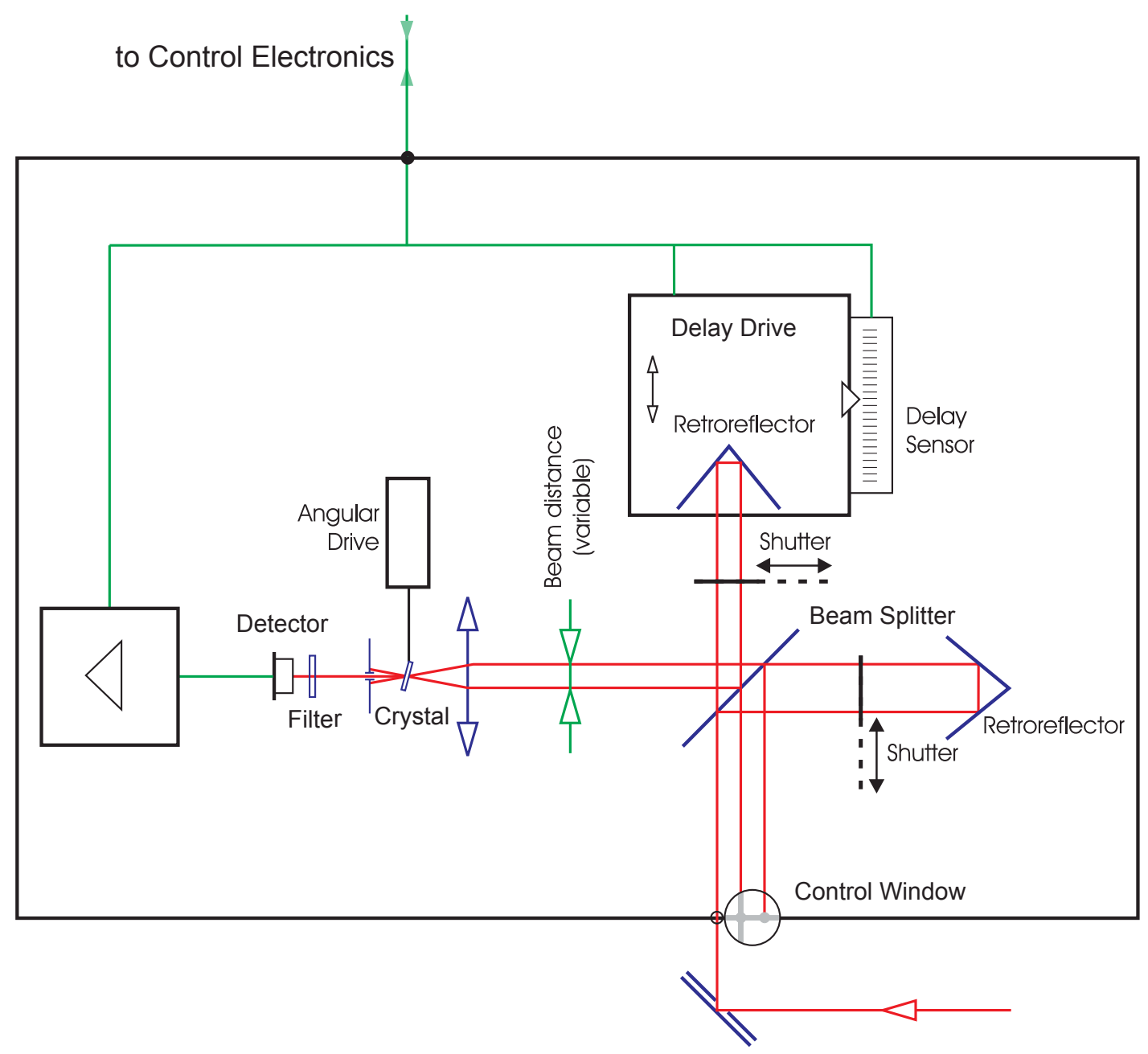

Abb. A.5: Skizze zum Aufbau des verwendeten Autokorrelators PulseScope des Herstellers APE - Angewandte Physik \& Elektronik GmbH (s. Abschnitt 4.3). 


\section{Symbolverzeichnis}

a Gitterparameter für die Breite der Gitterlücke

$b \quad$ Gitterparameter für die Breite des Gittersteges

$\mathrm{c}_{0} \quad$ Vakuumlichtgeschwindigkeit, $\mathrm{c}_{0}=299792458 \mathrm{~m} / \mathrm{s}$ (exakt)

c Ausbreitungs- bzw. Lichtgeschwindigkeit im Medium

$d_{p} \quad$ Eindringtiefe des evaneszenten Feldes

d Gittertiefe

$\partial \quad$ Differentialoperator der partiellen Ableitung

$\vec{E} \quad$ elektrisches (Vektor-)Feld mit den Richtungskomponenten $E_{x}, E_{y}$, $E_{z}$

$E_{0} \quad$ (komplexe) Amplitude des elektrischen Feldes

$\vec{E}_{p} \quad \vec{E}$-Feldanteil parallel zur Ausbreitungsrichtung

$\vec{E}_{s} \quad \vec{E}$-Feldanteil senkrecht zur Ausbreitungsrichtung

$\vec{e}_{x} \quad$ kanonischer Einheitsvektor in x-Richtung

$\vec{e}_{y} \quad$ kanonischer Einheitsvektor in y-Richtung

$\vec{e}_{z} \quad$ kanonischer Einheitsvektor in z-Richtung

$e \quad$ Elementarladung, $e=1,602176487(40) \cdot 10^{-19} \mathrm{~A} \cdot \mathrm{s}$

e EuLERsche Zahl

$\kappa \quad$ Extinktionskoeffizient der komplexen Brechzahl $\tilde{n}$

F $\quad$ Finessekoeffizient

$\mathcal{F} \quad$ Operator der kontinuierlichen FOURIER-Transformation

G Gangunterschied im Wellenleiter 
$\alpha \quad$ Absorptionskoeffizient

$\beta \quad$ Propagationskonstante

$\Delta \lambda \quad$ spektrale (Resonanz-)Bandbreite

$\Delta n_{\mathrm{wL}} \quad$ Brechzahländerung des Wellenleiters

$\Delta \tilde{\nu} \quad$ Frequenzlinienbreite in Wellenzahlen

$\delta \quad$ relativer Phasenversatz der Gitterstrukturen

$\Delta s \quad$ Wegunterschied

$\Delta \mathrm{T} \quad$ Temperaturänderung

$\Delta \theta \quad$ Resonanzwinkelbreite

$\Delta t \quad$ zeitliche Pulslänge

$\Delta \mathrm{V} \quad$ Änderung des Mischvolumens

$\varepsilon_{0} \quad$ elektrische Leitfähigkeit (Permittivität), $\varepsilon_{0}=1 /\left(\mu_{0} \mathrm{c}_{0}^{2}\right)=\mathrm{As} / \mathrm{Vm}$

$\eta \quad$ Quanteneffizienz

$\gamma \quad$ Dissipationskonstante der Leckwelle

$\lambda \quad$ Wellenlänge

$\lambda_{0} \quad$ Wellenlänge im Vakuum

$\Lambda \quad$ Gitterperiode

$\mu_{0} \quad$ magnetische Leitfähigkeit (Permeabilität), $\mu_{0}=4 \pi \cdot 10^{-7} \mathrm{Vs} / \mathrm{A} \mathrm{m}$

$\nu \quad$ Frequenz

$\tilde{\nu} \quad$ Wellenzahl

$\omega \quad$ Kreisfrequenz

$\omega_{0} \quad$ Kreisfrequenz im Vakuum

$\Phi_{10} \quad$ Phasensprung am Medienübergang $n_{1} / n_{0}$

$\Phi_{12} \quad$ Phasensprung am Medienübergang $n_{1} / n_{2}$

$\Phi \quad$ Phasenverschiebung bei Totalreflexion

$\Phi \overline{\text { DGWS }}$ Gesamtphasenverschiebung innerhalb der DGWS

$\Phi \overline{\text { DWG }}$ Gesamtphasenverschiebung innerhalb der $\overline{\mathrm{DWG}}$

$\Phi_{\overline{\text { FWHM }}}$ Phasenverschiebung bei $I\left(\Phi_{\overline{\mathrm{DWG}}}\right)=I_{0} / 2$

$\Phi_{G} \quad$ Phasenverschiebung durch Gangunterschied

$\Phi_{\mathrm{WL}} \quad$ Gesamtphasenverschiebung im Wellenleiter

$\pi \quad$ Kreiszahl

$\sigma_{\text {[TPA }} \quad$ Zwei-Photonen-Absorptionsquerschnitt

$\sigma_{\text {TPE }} \quad$ Wirkungsquerschnitt der Zwei-Photonen-Anregung 
$\tau \quad$ Korrelationszeit

$\tau_{\mathrm{c}} \quad$ Kohärenzzeit

$\tau_{\mathrm{R}} \quad$ zeitliche Kopplungslänge der Leckwelle

$\theta \quad$ Einfallswinkel

$\theta_{\mathrm{c}} \quad$ Grenzwinkel der Totalreflexion

$\theta_{\mathrm{R}} \quad$ Resonanzwinkel

$\mathrm{h} \quad$ PlanCKsches Wirkungsquantum, $\mathrm{h}=6,62606896(33) \cdot 10^{-34} \mathrm{~J} \cdot \mathrm{s}$

$h_{\text {eff }} \quad$ effektive Wellenleiterdicke

$\vec{H} \quad$ magnetisches (Vektor-)Feld mit den Richtungskomponenten $H_{x}$, $H_{y}, H_{z}$

$H_{0} \quad$ (komplexe) Amplitude des magnetischen Feldes

$\vec{H}_{P} \quad \vec{H}$-Feldanteil parallel zur Ausbreitungsrichtung

$\vec{H}_{S} \quad \vec{H}$-Feldanteil senkrecht zur Ausbreitungsrichtung

$h_{\text {Sub }} \quad$ Substratdicke

$h \quad$ Wellenleiterdicke

i $\quad$ imaginäre Einheit, $\mathrm{i}^{2}=-1$

I Intensität

$I_{0} \quad$ Intensität der einfallenden Welle

$I_{\mathrm{F}} \quad$ Intensität des reflektierten FRESNEL-Anteils

$I_{\mathrm{NR}} \quad$ Intensität der reflektierten Welle bei nicht-resonanter Anregung

$I_{R} \quad$ Intensität der reflektierten Welle

$I_{\mathrm{R}} \quad$ Intensität der resonanten Leckwelle

ITPF Intensität des Zwei-Photonen-Fluoreszenzsignals

$I_{T} \quad$ Intensität der transmittierten Welle

$\vec{K} \quad$ (reziproker) Gittervektor

$k \quad$ Kreiswellenzahl

$\vec{k} \quad$ (reziproker) Wellenvektor

$L_{\mathrm{GH}} \quad$ longitudinale Goos-HänCHEN-Verschiebung, $L_{\mathrm{GH}} \equiv L_{\times}$

$L_{\mathbf{x}} \quad$ longitudinale Verschiebung 
$L_{\mathbf{z}} \quad$ transversale Verschiebung

$l_{\mathrm{c}} \quad$ Kohärenzlänge

$l_{\mathrm{R}} \quad$ Kopplungslänge der resonanten Leckwelle

$m \quad$ Beugungsordnung

M Modenzahl: Anzahl der Schwingungsmoden

n relative Brechzahl

$n_{0} \quad$ Brechzahl im Superstrat

$n_{1} \quad$ Brechzahl im Wellenleiter

$n_{2} \quad$ Brechzahl im Substrat

$\tilde{n} \quad$ komplexer Brechungsindex

$n_{\mathrm{wL}} \quad$ Brechzahl des Wellenleiters, $n_{\mathrm{wL}} \equiv n_{1}$

$N \quad$ effektiver Modenindex

$R \quad$ Reflektivität

$R_{0} \quad$ Reflektivität 0 . Beugungsordnung

$\vec{r} \quad$ Ortsvektor

$\mathrm{T} \quad$ Temperatur (siehe Temperaturänderung $\Delta \mathrm{T}$ )

$T \quad$ Transmittivität

$T_{0} \quad$ Transmittivität 0. Beugungsordnung

$t \quad$ Zeitvariable

$T_{F} \quad$ Zeitfenster der Autokorrelation

VTPF Verstärkungsfaktor der Zwei-Photonen-Fluoreszenzanregung

$W_{\mathrm{p}} \quad$ Photonenenergie

$w \quad$ Standardabweichung

$W_{\text {TPE }} \quad$ Zwei-Photonen-Anregungsenergie 


\title{
Abkürzungsverzeichnis
}

\author{
AKF Autokorrelationsf unktion: Begriff aus der Signalverarbeitung \\ BBO $\quad \beta-\mathbf{B a B}_{2} \mathbf{O}_{4}$ : Beta-Bariumborat(-Kristall) \\ BMBF Bundesministerium für Bildung und Forschung: ein Bundesminis- \\ terium der Bundesrepublik Deutschland \\ CCD Charge-coupled device: engl. für ladungsgekoppeltes Bauteil \\ CMT Coupled-mode theory: Rechenmethode \\ CW Continuous wave: engl. für Dauerstrich(-Betrieb)
}

DGaO Deutsche Gesellschaft für angewandte Optik e. V.: Deutsche Niederlassung der European Optical Society

DGWS Double-grating waveguide structure: engl. für Doppel-GitterWellenleiter-Struktur

DOE Diffraktives optisches Element: optisches Element zur Formung eines Laserstrahls.

DUV Deep ultraviolet: engl. für tiefe ultraviolette Strahlung

DWG Dielektrisches Wellenleitergitter: planare Verbundstruktur aus Wellenleiter und Gitter

EBE Electron-beam evaporation: engl. für Elektronenstrahlverdampfen

EBPVD Electron beam physical vapor deposition: engl. für elektronenstrahlgestützte physikalische Gasphasenabscheidung

EMT Effective medium theory: Theorie zur Berechnung einer effektiven Brechzahl an periodischen Medien

EWS Evanescent wave scattering: engl. für Streuung evaneszenter Wellen 
FDTD Finite difference time domain: engl. für Finite-Differenzen-Methode im Zeitbereich

FEM Finite-Elemente-Methode: numerisches Rechenverfahren zur näherungsweisen Lösung

FPI Fabry-Pérot-Interferometer: optischer Resonator nach Charles Fabry und Alfred Pérot

FWHM Full width at half maximum: engl. für Halbwertsbreite

GDD Group delay dispersion: engl. für Gruppenlaufzeitdispersion

GMGR Guided-mode grating resonance: engl. Bezeichnung für die Resonanzeigenschaft einer DWG

GMR Guided-mode resonance: engl. Bezeichnung für die Resonanzeigenschaft einer $D W G$

GMRF Guided-mode resonance filter: engl. (anwendungsorientierte) Bezeichnung der Resonanzeigenschaft einer DWG

GVD Group velocity dispersion: engl. für Gruppengeschwindigkeitsdispersion

GWS Grating waveguide structure: engl. für Gitter-Wellenleiter-Struktur

HLA Human leukocyte antigen: engl. für menschl. Leukozytenantigen

IEEE Institute of Electrical and Electronics Engineers: Berufsverband von Ingenieuren aus den Bereichen Elektrotechnik und Informatik

IR Infrared: engl. für Infrarot

KKF Kreuzkorrelationsfunktion: Begriff aus der Signalverarbeitung.

LY Lucifer yellow: Fluoreszenzfarbstoff

MHC Major histocompatibility complex: engl. für Haupthistokompatibilitätskomplex 
NA Numerische Apertur: Maß für das Auflösungsvermögen von Optiken

Nd:YAG Neodymium-doped yttrium aluminium garnet: engl. für Neodymdotierter Yttrium-Aluminium-Granat(-Kristall)

NIR Near infrared: engl. für nahes Infrarot

OPA Optical parametric amplification: engl. für optisch parametrischer Verstärker

OPE One-photon excitation: engl. für Ein-Photonen-Anregung

OPF One-photon fluorescence: engl. für Ein-Photonen-Fluoreszenz

OPG Optical parametric generation: engl. für optisch parametrischer Generator

OPO Optical parametric oscillator: engl. für optisch parametrischer Oszillator

OSA Optical Society of America: wissenschaftliche Gesellschaft im Bereich der Optik und Photonik

OWLS Optical waveguide lightmode spectroscopy: engl. Bezeichnung für eine sensorische Nachweismethode von biochemischen Stoffen durch Anregung von Wellenleitermoden

PIAD Plasma-ion-assisted deposition: engl. für plasmaionengestütztes Aufdampfen

PML Perfectly matched layer: Absorbierenden Schicht-Randbedingung bei Simulationen der FEM

PMS Puls-Magnetron-Sputtern: Depositionsverfahren der PVD

PVD Physical vapor deposition: engl. für physikalische Gasphasenabscheidung

RCWA Rigorous coupled-wave analysis: engl. Bezeichnung für rigorose Beugungstheorie

REM Rasterelektronenmikroskop: dt. für Scanning electron microscope

RKM Rasterkraftmikroskop: dt. für Scanning force microscope

RWG Resonant waveguide grating: alias $D W G$ 
SHG Second harmonic generation: engl. für Frequenzverdopplng

SNOM Scanning nearfield optical microscope: engl. für optisches Rasternahfeldmikroskop

SPIE Society of Photo-Optical Instrumentation Engineers: wissenschaftliche Gesellschaft im Bereich der Optik und Photonik

TE Transverse electric: engl. für transversal-elektrisch

TEM Transverse electromagnetic: engl. für transversal-elektromagn.

TIR Total internal reflection: engl. für Totalreflexion

TIRF Total internal reflection fluorescence: Fluoreszenzanregung durch Totalreflexion

TIRFM Total internal reflection fluorescence microscopy: Methode der Mikroskopie

TM Transverse magnetic: engl. für transversal-magnetisch

TMR Tetramethyl-Rhodamin: Fluoreszenzfarbstoff

TPA Two-photon absorption: engl. für Zwei-Photonen-Absorption

TPE Two-photon excitation: engl. für Zwei-Photonen-Anregung

TPF Two-photon fluorescence: engl. für Zwei-Photonen-Fluoreszenz

UV Ultraviolet: engl. für Ultraviolett(-Strahlung)

VDE Verband Deutscher Elektrotechniker: Technisch-wissenschaftlicher Verband der Elektrotechnik, Elektronik und Informationstechnik

VDI Verein Deutscher Ingenieure: Vereinigung von Ingenieuren und Naturwissenschaftlern

VIS visueller Bereich: Bezeichnungen für das mit dem menschl. Auge sichtbare Lichtspektrum

VUV Vacuum ultraviolet: engl. für Vakuum-Ultraviolett(-Strahlung)

w. E. willkürliche Einheit: Größe wird einheitenlos angegeben

WIOS Wavelength-interrogated optical sensors: engl. Bezeichnung für eine spektroskopische Nachweismethode von biochemischen Stoffen 


\section{Abbildungsverzeichnis}

$1.1 \quad$ Schematische Darstellung einer Doppel-Gitter-Wellenleiter-Struktur. 2

2.1 Konvention für die positive Richtung von $\vec{k}, \vec{E}$ und $\vec{H}$ beim Medienübergang $n_{1}$ nach $n_{0}\left(n_{0}>n_{1}\right)$ im Fall von TM+ bzw. TEFPolarisation. 13

2.2 Skizze $\mathrm{zu}(2.16)$ mit $n_{1}>n_{2}$ für verschiedene Einfallswinkel $\theta_{1}^{\prime}>$ $\theta_{\mathrm{c}}>\theta_{1} . \mathrm{Ab} \theta_{\mathrm{c}}$ tritt Totalreflexion auf. . . . . . . . . . . . 14

2.3 Exponentieller Abfall des evaneszenten $\vec{E}$-Feldes an der Grenzfläche $(y=0)$. . . . . . . . . . . . . . . . . 15

2.4 Geometrisches Modell zur Ausbreitung einer Schwingungsmode im Wellenleiter der Brechzahl $n_{1}$. Aufgrund von TIR tritt an den Grenzflächen eine Verschiebung $L_{\mathrm{GH}}$ von $k_{1}$ auf. . . . . . . . . . . . 17

2.5 Geometrisches Modell zur Gitterbeugung. . . . . . . . . . . . . . . 21

2.6 Geometrisches Modell zur resonanten Ein- bzw. Auskopplung der +1 . Beugungsordnung $(m=1)$ eines dielektrischen Wellenleitergitters (DWG) mit einer Gitterstruktur oberhalb des Wellenleiters (GWS). . . . . . . . . . . . . . . . . . . 23

2.7 Darstellung vom Zusammenhang der Resonanzwellenlänge und

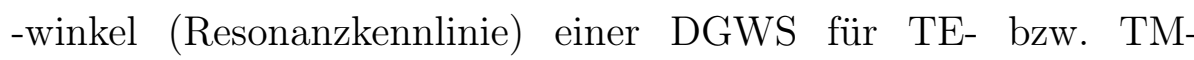
polarisiertes Licht. Ausschließlich im Propagationsbereich der Schwingungsmoden (Resonanzkorridor) ist eine Anregung der Moden durch die \pm 1 . Beugungsordnung möglich. . . . . . . . . . . . 39

2.8 Bei einem Resonanzwinkel von $-31,95^{\circ}$ berechnete Reflektivi-

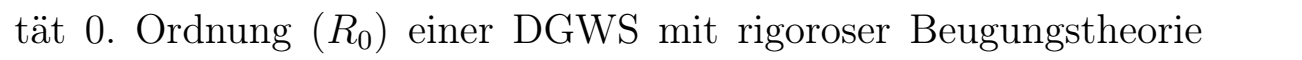
(RCWA) und analytischem Modell der Vielstrahlinterferenz (AIRY). 40

2.9 FEM-Simulationen zur E-Feldverteilung bei resonanter DGWS Anregung durch eine TE†polarisierte ebene Welle der Wellenlänge $800 \mathrm{~nm}$ unter $-31,82^{\circ}$ Einfall. . . . . . . . . . . . . . . . . . . . . . 41 


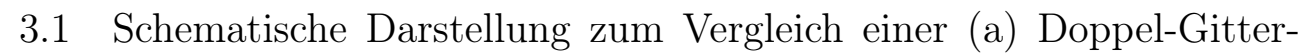

\begin{tabular}{|c|c|}
\hline & 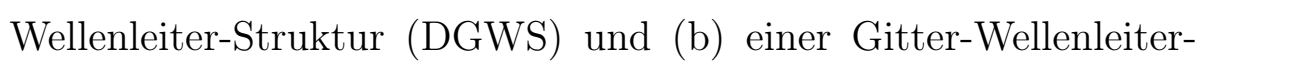 \\
\hline & Struktur (GWS). \\
\hline 3.2 & Aufbau der Bearbeitungsanlage zur $\mathrm{F}_{2}$-Laserablation. \\
\hline 3.3 & $\mathrm{~F}_{2}$-Laserablation erzeugte mittlere \\
\hline & den/Gittertiefen über der Pulszahl für verschiedene Fluenzen. \\
\hline 3.4 & \begin{tabular}{|l|l|l|} 
Mit einem & RKM & aufgenommene Oberflächenprofile der ablatierten \\
\end{tabular} \\
\hline & $\mathrm{Nb}_{2} \mathrm{O}_{5}$-Oberfläche $(\mathrm{a}) \mid$ in der Mitte und $(\mathrm{b}) \mid$ am Rand der Probe \\
\hline & GWS/Nb150/42. \\
\hline 3.5 & REM-Aufnahme von der mittels $\mathrm{F}_{2}$-Laserablation strukturierten \\
\hline & Oberfläche der Probe GWS/Nb150/42. \\
\hline 3.6 & \begin{tabular}{|l|l|l|l} 
Mit einem & RKM & aufgenommenes dreidimensionales $2 \times 2 \mu \mathrm{m}^{2}$ großes \\
\end{tabular} \\
\hline & \begin{tabular}{|l|l|l|} 
Abbild der Oberflächenstruktur: & (a) & zeigt die Gitterstruktur eines \\
\end{tabular} \\
\hline & unbeschichteten Substrats mit einer Gitterperiode $318 \mathrm{~nm}$ und einer \\
\hline & \begin{tabular}{|l|l|l|l} 
Gittertiefe $12 \mathrm{~nm}$ (Quelle: UNAXIS), (b) & zeigt die Oberfläche der \\
\end{tabular} \\
\hline & Probe DGWS/Ta150/24 nach der Beschichtung des Substrats. \\
\hline
\end{tabular}

4.1 Die nach Auswertung der Spektrometerdaten bestimmten $T_{0^{-}}$ \begin{tabular}{|l|l|l|l|}
\hline Resonanzkennlinien der Probe DGWS/Ta150/40 für & TE & bzw. TM-
\end{tabular}

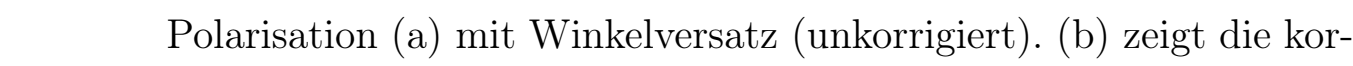

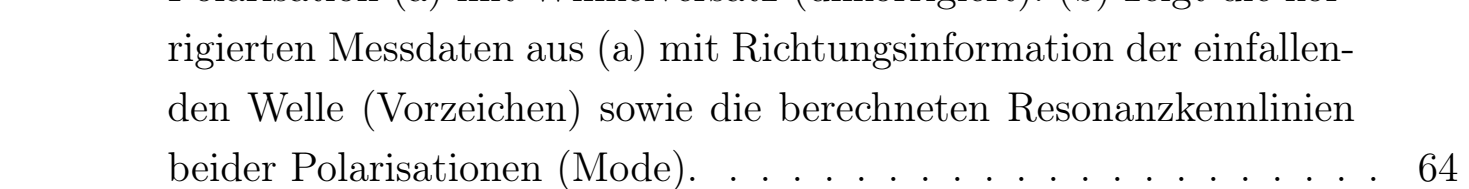

4.2 Messanordnung zur goniometrischen Charakterisierung der Proben in Reflexion und Transmission. . . . . . . . . . . . . . . . . . . . . . 67

4.3 Gemessenes (Daten) und berechnetes (RCWA) Winkelspektrum der

\begin{tabular}{|l|l|l|l|l|}
\hline Reflexion nullter Ordnung der & (a) & Probe DGWS/Hf30/18 bzw. & (b) & \\
\hline
\end{tabular}
DGWS/Hf30/40 für TE-polarisiertes Licht der Wellenlänge $266 \mathrm{~nm}$. . 69

4.4 Aufgenommene (Daten) und berechnete (RCWA) Intensitätsver-

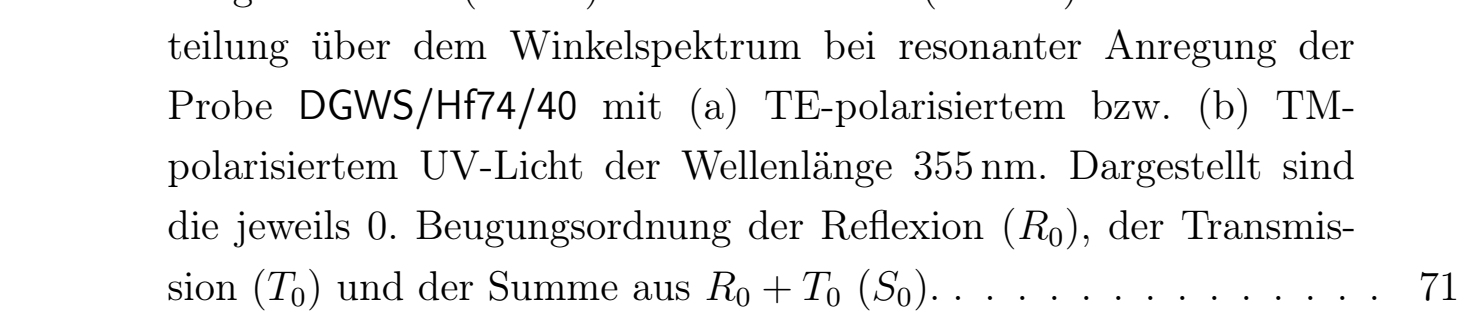


4.19 Mittels Autokorrelator aufgenommenes zeitabhängiges Korrelati-

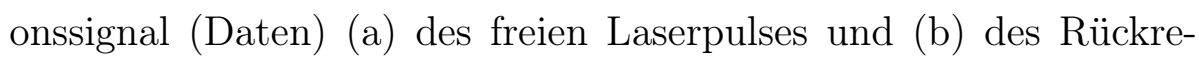
flexes der Probe DGWS/Ta150/24 (nicht-resonant). Die Anpassung der Messdaten erfolgt über die Fitfunktion $S_{\text {AKF }}$. . . . . . . . . . 93

4.20 Mittels Autokorrelator aufgenommenes zeitabhängiges Korrelationssignal (Daten) |(a) $\mid$ der Probe DGWS/Ta150/40 und|(b)|der Probe DGWS/Ta150/24. Die Anpassung der Messdaten erfolgt über die Fitfunktion $S_{\text {AKF, }}$ wobei die einzelnen Korrelationsterme (FRESNEL, Leckwelle und Mischterm) separat dargestellt sind. . . . . . . . . . 96

4.21 Mittels Spektrometer aufgenommene OPOfMischwellen: Signal-

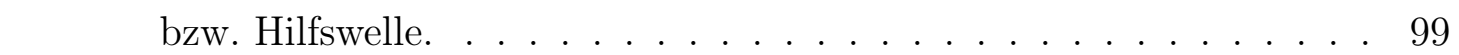

4.22 Mittels Spektrometer aufgenommene Farbstofflaserlinie (schwarz). Zusätzlich ist die zur Entfaltung des Spektrums benötigte Unschärfefunktion (grün) als GAUSS-Puls sowie das entfaltete Spektrum der Laserlinie (rot) dargestellt. . . . . . . . . . . . . . . . 101

5.1 Skizze zur Anregung von Farbstoffmolekülen auf der Oberfläche \begin{tabular}{|r|l|l|l|l}
\hline einer & DGWS & bei resonanter Bestrahlung über die Rückseite. Das
\end{tabular} \begin{tabular}{|c|c|}
\hline evaneszente Feld ist hier über eine & TEb-Mode im Bereich der wel- \\
\hline
\end{tabular}

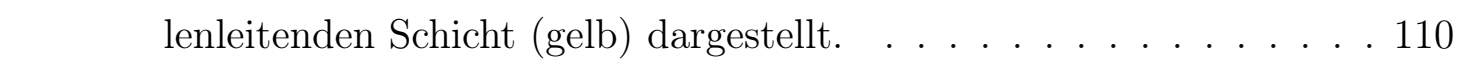

\begin{tabular}{l|l|l|}
5.2 & REM-Aufnahme vom Rand eines TMR-Films auf der Oberfläche \\
\hline
\end{tabular} einer GWS (Quelle: KATCHALSKI u. a.||2005b). . . . . . . . . . . . . 113

5.3 Messaufbau mit Strahlengang zur TPF mit Femtosekunden-Laserpulsen. . . . . . . . . . . . . . . . . 114

5.4 CCD-Kamerabild einer TPF-Anregung auf einer DGWStOberfläche. 115

\begin{tabular}{ll|l}
5.5 & Winkelabhängiges & TPFFSignal mittels Ti:Saphir-Laserpulsen an-
\end{tabular} \begin{tabular}{|lllll|}
\hline geregter Rhodamin-B-Moleküle auf der Oberfläche der Probe \\
\hline
\end{tabular} DGWS/Ta150/40. . . . . . . . . . . . . . . . . 116

5.6 CCD-Signal resonant (oben) bzw. nicht-resonant angeregter Rhodamin-B-Moleküle der Konzentration $\sim 10^{-12} \mathrm{~mol} / \mathrm{cm}^{2}$ auf der Oberfläche der Probe DGWS/Ta150/40 für verschiedene Laserleistungen. . . . . . . . . . . . . . . . . 117

5.7 Logarithmische Darstellung des Fluoreszenzlichts (TPF-Signal) aus Abb. 5.6 zur Bestimmung der $\mid$ TPFFVerstärkung. . . . . . . . . . . . 118 
5.8 FEM-Simulationen zur Energiedichte bei Bestrahlung der Probe

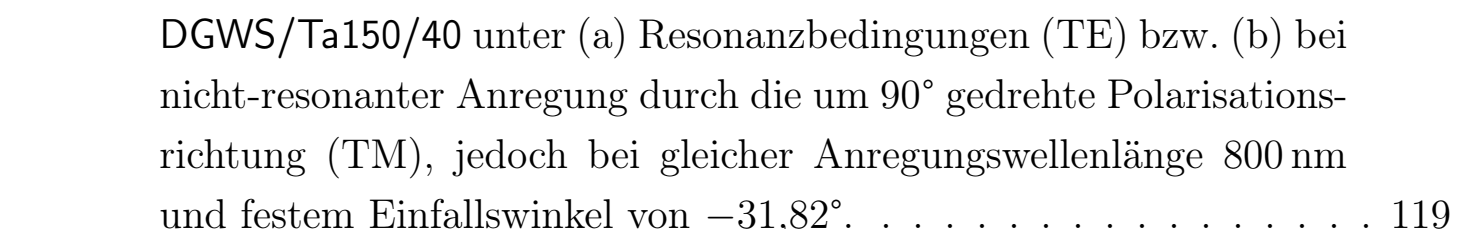

\begin{tabular}{|lll|ll|}
5.9 & Zwölf vertikale Schnittlinien entlang der & DGWS-Oberfläche $\mathrm{zu}$ \\
\hline
\end{tabular} Abb. 5.8 zeigen die Energiedichteverteilung bei resonanter bzw. nicht-resonanter Anregung. . . . . . . . . . . . . . . . . . . . 120

5.10 Messaufbau mit Strahlengang zur TPF Anregung von Fluorophoren mit Pikosekunden-Laserpulsen. . . . . . . . . . . . . . . . . 123

5.11 CCD-Signal angeregter LYFFarbstoffmoleküle auf der Oberfläche der Probe DGWS/Ta150/40 in den (gelösten) Konzentrationen (a) $10^{-4} \mathrm{~mol} / \mathrm{dm}^{-3}$ bzw. (b) $10^{-9} \mathrm{~mol} / \mathrm{dm}^{-3}$ bei verschiedenen Laserleistungen. . . . . . . . . . . . . . . . . . . 125

5.12 Skizze der mit Wasser $\left(\mathrm{H}_{2} \mathrm{O}\right)$ gefüllten Küvette, welche zur

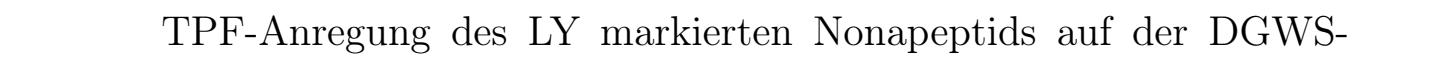
Oberfläche verwendet wird. . . . . . . . . . . . . . . . 126

5.13 CCD-Signal angeregter LY LFmarkierter Nonapeptide in der Konzentration $8 \cdot 10^{-12} \mathrm{~mol} / \mathrm{mm}^{2}$ auf der Oberfläche der innerhalb der Küvette arretierten DGWS bei verschiedenen Laserleistungen. . . . . . 127

A.1 Herstellerangaben zum SchOTT ${ }^{\circledR}{ }_{\text {-Glas AF } 45 .}$. . . . . . . . . . . . . . 133

A.2 Herstellerangaben zum SCHOTT ${ }^{\circledR}$-Glas D 263 T. . . . . . . . . . . . . . 134

A.3 $\quad$ Herstellerangaben zum UnAXIS-Filter Calflex $X^{\top m}$. . . . . . . . . . . . . 134

A.4 16 Spektrometer-Datensätze der Probe DGWS/Ta150/40 für verschiedene Einfallswinkel $\left(0^{\circ}-75^{\circ}\right)$ zur Bestimmung der TE

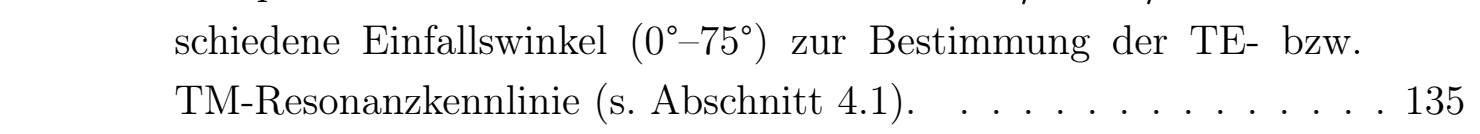

A.5 Skizze zum Aufbau des verwendeten Autokorrelators PulseScope des Herstellers APE - ANGEWANDTE PHYSIK \& ELEKTRONIK GmbH (s. Abschnitt 4.3). . . . . . . . . . . . . . . . . . . . . 136 



\section{Tabellenverzeichnis}

\begin{tabular}{|ll|l|}
\hline 3.1 Übersicht zu den Strukturdaten aller verwendeten & DWGłProben. \\
\hline Die angegebene Wellenleiterdicke entspricht der Beschichtungsdicke \\
\hline und unterscheidet sich daher von . . . . . . . . . . . . . . . 59
\end{tabular}

4.1 Probenübersicht mit (Design-)Wellenlänge und zugehörigem, angestrebtem Resonanzwinkel (Abschätzung) unter Angabe der Laserstrahlquelle und Polarisation. . . . . . . . . . . . . . . . . . . . . 62

4.2 Experimentell bestimmte Resonanzwinkel unter Angabe der verwendeten Laserstrahlquelle, Wellenlänge und Polarisation. . . . . . 103

4.3 Experimentell bestimmte (Daten) sowie berechnete (RCWA) Reflektivitäten $R_{0}$ bzw. Transmittivität $T_{0}$ aller Proben unter Angabe der verwendeten Wellenlänge und Polarisation. . . . . . . . . . . . . 104

4.4 Experimentell bestimmte Resonanzbandbreite bzw. Resonanzwinkelbreite aller Proben unter Angabe der verwendeten Wellenlänge und Polarisation. Die in Klammern gesetzten Werte entsprechen der jeweils umgerechneten Resonanzbreite. . . . . . . . . . . . . . 106 



\section{Literaturverzeichnis}

\section{Adams 1981}

Adams, Michael J.: An Introduction to Optical Waveguides. Chichester [u. a.] : John WilEy \& Sons, 1981. - ISBN 0-471-27969-2

$\rightarrow$ Zitiert auf Seite 27 .

\section{Albota u. a. 1998}

Albota, Marius A. ; Xu, Chris ; Webb, Watt W.: Two-photon fluorescence excitation cross sections of biomolecular probes from 690 to $960 \mathrm{~nm}$. In: Applied Optics 37 (1998), Nr. 31, S. 7352-7356

$\rightarrow$ Zitiert auf Seite 112

\section{Artmann 1942}

Artmann, Kurt: Zur Theorie der anomalen Reflexion von optischen Strichgittern. In: Zeitschrift für Physik A 119 (1942), Nr. 9-10, S. 529-567

$\rightarrow$ Zitiert auf Seite 4 .

\section{Artmann 1948}

Artmann, Kurt: Berechnung der Seitenversetzung des totalreflektierten Strahles. In: Annalen der Physik 437 (1948), Nr. 1-2, S. 87-102

$\rightarrow$ Zitiert auf Seite 16.

\section{Avrutsky u. a. 1989}

Avrutsky, I. A. ; Svakhin, A. S. ; Sychugov, V. A.: Interference phenomena in waveguides with two corrugated boundaries. In: Journal of Modern Optics 36 (1989), Nr. 10, S. 1303-1320

$\rightarrow$ Zitiert auf den Seiten 30, 31, 35 und 85. 


\section{Avrutsky und Sychugov 1989}

Avrutsky, I. A. ; Sychugov, V. A.: Reflection of a beam of finite size from a corrugated waveguide. In: Journal of Modern Optics 36 (1989), Nr. 11, S. $1527-1539$

$\rightarrow$ Zitiert auf Seite 31.

\section{AVRUTSKY und RABADY 2001}

Avrutsky, Ivan ; RABADY, Rabi: Waveguide grating mirror for large-area semiconductor lasers. In: Optics Letters 26 (2001), Nr. 13, S. 989-991

$\rightarrow$ Zitiert auf Seite 6 .

\section{Axelrod u. a. 1984}

Axelrod, Daniel ; Burghardt, Thomas P. ; Thompson, Nancy L.: Total internal reflection fluorescence. In: Annual Review of Biophysics and Bioengineering 13 (1984), S. 247-268

$\rightarrow$ Zitiert auf Seite 109 .

Axelrod u.a. 1992

Axelrod, Daniel ; Hellen, Edward H. ; Fulbright, Robert M.: Total Internal Reflection Fluorescence. In: LAKOWICZ, Joseph R. (Hrsg.): Topics in Fluorescence Spectroscopy. Bd. 3 : Biochemical Applications. New York: Plenum Press, 1992, Kapitel 7. - ISBN 0-306-43954-9, S. 289-337

$\rightarrow$ Zitiert auf den Seiten 15 und 109

BADER u. a. 2004

Bader, M. A. ; Kappel, C. ; Selle, A. ; Ihlemann, J. ; NG, M. L. ; HerMAN, P. R.: Fabrication of sub-micron gratings in ultrathin films by $157-\mathrm{nm}$ laser ablation and their application as grating waveguide structures. In: ARmitage, J. C. (Hrsg.) ; Lessard, R. A. (Hrsg.) ; Lampropoulos, G. A. (Hrsg.): Proceedings of SPIE (Photonics North 2004, Ottawa, Ontario, Kanada, 27.--29. September 2004). Bd. 5578 : Photonic Applications in Astronomy, Biomedicine, Imaging, Materials Processing, and Education. Bellingham (Washington) : SPIE Press, Dezember 2004. - ISBN 0-8194-5533-4, S. 559-567

$\rightarrow$ Zitiert auf den Seiten 51 und 107. 
BADER u. a. 2006

Bader, M. A. ; Kappel, C. ; Selle, A. ; Ihlemann, J. ; NG, M. L. ; HERMAN, P. R.: $\mathrm{F}_{2}$-laser-machined submicrometer gratings in thin dielectric films for resonant grating waveguide applications. In: Applied Optics 45 (2006), Nr. 25, S. 6586-6590

$\rightarrow$ Zitiert auf den Seiten 52 und 107.

\section{BADER u. a. 2007}

Bader, M. A. ; Selle, A. ; Stenzel, O. ; Delmdahl, R. ; Spiecker, G. ; FisCHER, C.: High spectral resolution analysis of tunable narrowband resonant grating waveguide structures. In: Applied Physics B : Lasers and Optics 89 (2007), Nr. 2-3, S. 151-154

$\rightarrow$ Zitiert auf Seite 85

\section{BADER 2001}

BADER, Mark A.: Erzeugung und Charakterisierung von gitter-strukturierten Polymer-Wellenleitern für nichtlinear-optische Schaltprozesse. Göttingen, Universität, Fakultät für Physik, Diss., 2001. - ISBN 3-89873-278-9

$\rightarrow$ Zitiert auf den Seiten 6 und 45 .

\section{BADER 2003}

BADER, Mark A.: Gitter-Wellenleiter-Strukturen aus Polymeren für optische Schalter / Laser-Laboratorium Göttingen e.V. Göttingen, Mai 2003 (Förderkennzeichen 03 N 8634 A). - Forschungsbericht. - Teilvorhaben im BMBF-Förderprogramm: Interdisziplinäre Materialforschung mit Nachwuchswissenschaftlerinnen und Nachwuchswissenschaftlern - Hot Topics, VerbundNr. 01020325, Abschlussbericht

$\rightarrow$ Zitiert auf den Seiten 6 und 45 . 


\section{BADER 2006}

Bader, Mark A. ; VDI VDEInnovation + TEChnik GmbH (Hrsg.): Herstellung und Charakterisierung von Gitter-Wellenleiter-Strukturen für den Einsatz als Superspiegel - SPOT / Laser-Laboratorium Göttingen e. V. Berlin, Juni 2006 (Förderkennzeichen 16 IN 0149). - Forschungsbericht. - Teilvorhaben im BMBFFFörderprogramm: Förderung von innovativen Netzwerken InnoNet, Verbund-Nr. 01022910, Abschlussbericht

$\rightarrow$ Zitiert auf Seite 107

\section{BAO und HuANG 2004}

Bao, Gang ; Huang, Kai: Optimal Design of Guided-Mode Grating Resonance Filters. In: IEEE Photonics Technology Letters 16 (2004), Nr. 1, S. $141-144$

$\rightarrow$ Zitiert auf Seite 3

\section{BAO und HuANG 2005}

BaO, Gang ; HuAng, Kai: Computational design for guided-mode grating resonances. In: Journal of Optical Society of America A 22 (2005), Nr. 7, S. $1408-1413$

$\rightarrow$ Zitiert auf Seite 3 ,

\section{BEAMLUX II 2005}

BeamLux II. 2.3.4 u. 2.4.3. Metrolux GmbH, 2005. - Software, 32-BitProgramm für Windows ${ }^{\circledR}$-Betriebssysteme

$\rightarrow$ Zitiert auf Seite 82 .

\section{BENDICKSON u.a. 2001}

Bendickson, J. M. ; Glytsis, E. N. ; Gaylord, T. K. ; Brundrett, D. L.: Guided-mode resonant subwavelength gratings: effects of finite beams and finite gratings. In: Journal of Optical Society of America A 18 (2001), Nr. 8, S. $1912-1928$

$\rightarrow$ Zitiert auf Seite 106 . 


\section{BERENGER 1994}

BerEnger, Jean-Pierre: A perfectly matched layer for the absorption of electromagnetic waves. In: Journal of Computational Physics 114 (1994), Nr. 2,

S. $185-200$

$\rightarrow$ Zitiert auf Seite 42 .

\section{BLOCK 2005}

BLOCK, Stephan: Untersuchung neuer Verfahren zur Frequenzdurchstimmung von External-Cavity-Diodenlasern. Greifswald, Universität, Diplomarbeit, Juni 2005

$\rightarrow$ Zitiert auf Seite 132

\section{BORN und WOLF 1999}

Born, Max ; Wolf, Emil: Principles of Optics : Electromagnetic Theory of Propagation, Interference and Diffraction of Light. 7., erw. Aufl. Cambridge [u. a.] : Cambridge University Press, 1999. - ISBN 0-521-64222-1

$\rightarrow$ Zitiert auf den Seiten 10, 11, 14, 16, 17, 37, und 101.

\section{BOYD 2008}

Boyd, Robert W.: Nonlinear Optics. 3. Aufl. Oxford : Academic Press / Elsevier, Inc., 2008. - ISBN 978-0-12-369470-6

$\rightarrow$ Zitiert auf Seite 66.

\section{Boye und Kostuk 2000}

Boye, Robert R. ; Kostuk, Raymond K.: Investigation of the effect of finite grating size on the performance of guided-mode resonance filters. In: Applied Optics 39 (2000), Nr. 21, S. 3649-3653

$\rightarrow$ Zitiert auf den Seiten 98 und 106 .

BOYE u. a. 1999

Boye, Robert R. ; Ziolkowski, Richard W. ; Kostuk, Raymond K.: Resonant waveguide-grating switching device with nonlinear optical material. In: Applied Optics 38 (1999), Nr. 24, S. 5181-5185

$\rightarrow$ Zitiert auf den Seiten 6 und 45 , 
Brazas u. a. 1995

Brazas, John C. ; Li, Lifeng ; MCKeon, Amanda L.: High-efficiency input coupling into optical waveguides using gratings with double-surface corrugation. In: Applied Optics 34 (1995), Nr. 4, S. 604-609

$\rightarrow$ Zitiert auf den Seiten 85 und 106 .

BUDACH u. a. 2003

Budach, W. ; Neuschäfer, D. ; Wanke, C. ; Chibout, S.: Generation of transducers for fluorescence-based microarrays with enhanced sensitivity and their application for gene expression profiling. In: Analytical Chemistry 75 (2003), Nr. 11, S. 2571-2577

$\rightarrow$ Zitiert auf den Seiten 7 und 110 .

\section{Budach und Neuschaefer 2001}

Schutzrecht WO 01/02839-A1 (Januar 2001). BudACH, Wolfgang Ernst G. ; Neuschaefer, Dieter (Erfinder); Novartis AG (Anmelder). Pr.: GB 9915703 1999-07-05 / GB 0011420 2000-05-11. - Sensor platform, apparatus incorporating the platform, and process using the platform

$\rightarrow$ Zitiert auf den Seiten 6 und 131 .

\section{BUNKOWSKI u. a. 2006}

Bunkowski, A. ; Burmeister, O. ; Friedrich, D. ; Danzmann, K. ; SchnABEL, R.: High reflectivity grating waveguide coatings for $1064 \mathrm{~nm}$. In: Classical and Quantum Gravity 23 (2006), Nr. 24, S. 7297-7303

$\rightarrow$ Zitiert auf Seite 42 .

Challener u. a. 2000

Challener, W. A. ; Edwards, J. D. ; McGowan, R. W. ; Skorjanec, J. ; YANG, Z.: A multilayer grating-based evanescent wave sensing technique. In: Sensors and Actuators B : Chemical 71 (2000), Nr. 1-2, S. 42-46

$\rightarrow$ Zitiert auf Seite 109 .

\section{COMSOL 2008}

COMSOL Multiphysics ${ }^{\circledR}$. 3.4. COMSOL, Inc., 2008. - Software, 64-BitProgramm für Windows ${ }^{\circledR}$-Betriebssysteme

$\rightarrow$ Zitiert auf Seite 38 . 


\section{Cottien u. a. 2002}

Cottier, K. ; Kunz, R. E. ; Voirin, G. ; Wiki, M.: Thickness-modulated waveguides for integrated optical sensing. In: Gannot, I. (Hrsg.): Proceedings of SPIE (International Biomedical Optics Symposium - BiOS 2002, San José, Kalifornien, USA, 19.-25. Januar 2002). Bd. 4616: Optical Fibers and Sensors for Medical Applications II. Bellingham (Washington) : SPIE Press, März 2002. - ISBN 0-8194-4355-7, S. 53-63

$\rightarrow$ Zitiert auf den Seiten 47 und 54 .

\section{DAKSS u.a. 1970}

Dakss, M. L. ; Kuhn, L. ; Heidrich, P. F. ; Scott, B. A.: Grating coupler for efficient excitation of optical guided waves in thin films. In: Applied Physics Letters 16 (1970), Nr. 12, S. 523-525

$\rightarrow$ Zitiert auf den Seiten 3 und 6 .

\section{DEEGAN u.a. 1997}

Deegan, R. D. ; Bakajin, O. ; Dupont, T. F. ; Huber, G. ; Nagel, S. R. ; Witten, T. A.: Capillary flow as the cause of ring stains from dried liquid drops. In: Nature 389 (1997), S. 827-829

$\rightarrow$ Zitiert auf Seite 114

DelmDAhl u. a. 2007

Delmdahl, R. F. ; Spiecker, G. ; Bader, M. A. ; Selle, A.: Spectral resolution analysis of resonant grating waveguides. In: Materialwissenschaft und Werkstofftechnik 38 (2007), Nr. 3, S. 218-221

$\rightarrow$ Zitiert auf Seite 85 .

\section{DEMTRÖDER 2007}

DemtröDER, Wolfgang: Laserspektroskopie : Grundlagen und Techniken. 5., erw. und neubearb. Aufl. Berlin [u. a.] : SpRInger-Verlag, 2007. - ISBN 3540-33792-X

$\rightarrow$ Zitiert auf den Seiten 92 und 93 . 


\section{Diels und RudolPh 1996}

Diels, Jean-Claude ; Rudolph, Wolfgang ; LiaO, Paul F. (Hrsg.) ; Kelley, Paul (Hrsg.) ; Kaminow, Ivan (Hrsg.): Ultrashort Laser Pulse Phenomena : Fundamentals, Techniques, and Applications on a Femtosecond Time Scale. San Diego [u. a.] : Academic Press, 1996 (Optics and Photonics Series). - ISBN 0-12-215492-4

$\rightarrow$ Zitiert auf den Seiten 93 und 96 .

DONVAL u. a. 2001

Donval, A. ; Toussaere, E. ; Zyss, J. ; Levy-Yurista, G. ; Jonsson, E. ; FRIESEM, A. A.: Novel polymer-based resonant grating-waveguide structures. In: Synthetic Metals 124 (2001), Nr. 1, S. 19-22

$\rightarrow$ Zitiert auf Seite 6 .

\section{DreXHAGe 1990}

Drexhage, Karl H.: Structure and properties of laser dyes. In: Schäfer, Fritz P. (Hrsg.): Topics in Applied Physics. Bd. 1 : Dye Lasers. 3., erw. und überarb. Aufl. Springer-Verlag, 1990, Kapitel 5. - ISBN 3-540-51558-5, S. $155-200$

$\rightarrow$ Zitiert auf Seite 117

Dudovich u. a. 2001

Dudovich, N. ; Levy-Yurista, G. ; Sharon, A. ; Friesem, A. A. ; Weber, H. G.: Active semiconductor-based grating waveguide structures. In: IEEE Journal of Quantum Electronics 37 (2001), Nr. 8, S. 1030-1039

$\rightarrow$ Zitiert auf den Seiten 6 und 45.

\section{DUVENECK u. a. 2001a}

Schutzrecht WO 01/079821 (Oktober 2001). DuvenECK, G. ; BopP, M. ; Pawlak, M. ; Ehrat, M. ; Marowsky, G. (Erfinder); Zeptosens AG (Anmelder). Pr.: CH 742/00 2000-04-14. - Gitter-Wellenleiter-Struktur zur Verstärkung eines Anregungsfeldes und deren Verwendung

$\rightarrow$ Zitiert auf den Seiten 3 und 6 . 
DUVENECK u. a. 2001b

Duveneck, G. L. ; Bopp, M. A. ; Ehrat, M. ; Haiml, M. ; Keller, U. ; Bader, M. A. ; Marowsky, G. ; Soria, S.: Evanescent-field-induced twophoton fluorescence: excitation of macroscopic areas of planar waveguides. In: Applied Physics B : Lasers and Optics 73 (2001), Nr. 8, S. 869-871

$\rightarrow$ Zitiert auf Seite 8 .

\section{EBERT und EBERT 2002}

EBERT, Martin ; EBERT, Johannes: Magnetron-Sputtern im Mikrowellenplasma im Vergleich zu konventionellen Aufdampfverfahren. In: Photonik : Fachzeitschrift für die Optischen Technologien 1 (2002), S. 34-37

$\rightarrow$ Zitiert auf den Seiten 7, 53, 90 und 107.

\section{EICHLER und EICHLER 2006}

EichleR, Jürgen ; EichleR, Hans J.: Laser : Bauformen, Strahlführung, Anwendungen. 6., aktualisierte Aufl. Berlin [u. a.] : SPRINGER-Verlag, 2006. ISBN 3-540-30149-6

$\rightarrow$ Zitiert auf den Seiten 67, 74 und 98 .

\section{Escoubas und Flory 2003}

Escoubas, Ludovic ; Flory, Francois: Optical Thin Films for MicroComponents. In: KaIser, Norbert (Hrsg.) ; Pulker, Hans K. (Hrsg.): Springer Series in Optical Sciences. Bd. 88 : Optical Interference Coatings. Berlin, Heidelberg : SPRINGER-Verlag, 2003. - ISBN 3-540-00364-9, S. 231-255

$\rightarrow$ Zitiert auf Seite 52 .

\section{FABRY und PÉRot 1899}

FABRY, Charles ; PÉROT, Alfred: Théorie et applications d'une nouvelle méthode de spectroscopie interférentielle. In: Annales de Chimie et de Physique 16 (1899), Nr. 7, S. 115-144

$\rightarrow$ Zitiert auf Seite 36 .

FANG u. a. 2006

Fang, Y. ; Ferrie, A. M. ; Fontaine, N. H. ; Mauro, J. ; Balakrishnan, J.: Resonant waveguide grating biosensor for living cell sensing. In: Biophysical Journal 91 (2006), Nr. 5, S. 1925-1940

$\rightarrow$ Zitiert auf den Seiten 3 und 131 . 


\section{FEDOROV 1955}

Fedorov, Fedor I.: K теории полного отражениа. In: Doklady Akademii Nauk SSSR 105 (1955), Nr. 3, S. 465-468

$\rightarrow$ Zitiert auf Seite 16 .

\section{FIEBIG 2001}

FieBIG, Michael: LPF ${ }^{\text {TM }}$ Series. In: BASTing, Dirk (Hrsg.): Excimer Laser Technology : Laser Sources, Optics, Systems and Applications. Göttingen : Lambda Physik AG, 2001, Kapitel 4. - ISBN 3-00-006395-1, S. 271-274

$\rightarrow$ Zitiert auf Seite 48 .

\section{FISCHER u. a. 1995}

Fischer, Andreas ; Cremer, Christoph ; Stelzer, Ernst H. K.: Fluorescence of coumarins and xanthenes after two-photon absorption with a pulsed titanium-sapphire laser. In: Applied Optics 34 (1995), Nr. 12, S. 1989-2003

$\rightarrow$ Zitiert auf den Seiten 112 und 116 .

\section{FLUCKIGER 2004}

Fluckiger, David: GSOLVER ${ }^{\odot}$. 4.20c. Grating Solver DevelopMent Company, 2004. - Demo-Software, 16-Bit-Programm für Windows ${ }^{\circledR}$ Betriebssysteme

$\rightarrow$ Zitiert auf Seite 38.

\section{DE ForNel 2001}

Fornel, Frédérique de ; Rhodes, W. T. (Hrsg.) ; Asakura, T. (Hrsg.) ; Brenner, K.-H. (Hrsg.) ; Hänsch, F. T. W. T. W. nad Krausz K. T. W. nad Krausz (Hrsg.) ; WeBER, H. (Hrsg.): Springer Series in Optical Sciences. Bd. 73: Evanescent Waves : From Newtonian Optics to Atomic Optics. Berlin [u. a.] : SPRINGER-Verlag, 2001. - ISBN 3-540-65845-9

$\rightarrow$ Zitiert auf Seite 15 . 


\section{FRICKE-BEGEMANN u. a. 2005}

Fricke-Begemann, T. ; Bader, M. A. ; Ihlemann, J. ; Kappel, C. ; Meinertz, J. ; SElle, A.: Two-photon fluorescence : large area excitation and enhanced sensitivity using waveguide structures. In:DGaO-Proceedings (106. Joint Conference of German Society of Applied Optics DGaO and Section of Optics of the Polish Physical Society, Wroclaw, Polen, 17.-21. Mai 2005), 2005. - Art. B24. - URL http://www.dgao-proceedings.de/download/106/ 106_b24.pdf

$\rightarrow$ Zitiert auf Seite 118

\section{FRIESEM u. a. 2002}

Friesem, A. A. ; Dudovich, N. ; Katchalski, T. ; Levy-Yurista, G.: Grating waveguide structures and their application. In: CAUlfield, H. J. (Hrsg.): Proceedings of SPIE (AeroSense 2002 : Sensor Technology and Applications, Orlando, Florida, USA, 3.-4. April 2002). Bd. 4737 : Holography : A Tribute to Yuri Denisyuk and Emmett Leith. Bellingham (Washington) : SPIE Press, Juli 2002. - ISBN 0-8194-4503-7, S. 108-115

$\rightarrow$ Zitiert auf Seite 6 .

\section{Friesem und SHARON 1999}

Schutzrecht EP 0898 799-B1 (März 1999). Friesem, Asher A. ; Sharon, Avner Z. (Erfinder); Yeda Research and Development Company Ltd. (Anmelder). Pr.: IL 11820996 1996-05-09. - Active wavelength selection with resonant devices

$\rightarrow$ Zitiert auf Seite 6 .

\section{Glasberg u.a. 1998}

Glasberg, S. ; Sharon, A. ; Rosenblatt, D. ; Friesem, A. A.: Spectral shifts and line-shapes asymmetries in the resonant response of grating waveguide structures. In: Optics Communications 145 (1998), Nr. 1-6, S. 291-299

$\rightarrow$ Zitiert auf Seite 6 . 
Golubenko u. a. 1986

Golubenko, G. A. ; Svakhin, A. S. ; Sychugov, V. A. ; Tischenko, A. V. ; Popov, E. ; MAshev, L.: Diffraction characteristics of planar corrugated waveguides. In: Optical and Quantum Electronics 18 (1986), Nr. 2, S. 123-128

$\rightarrow$ Zitiert auf Seite 5 .

Golubenko u.a. 1985

Golubenko, G. A. ; Svakhin, A. S. ; Sychugov, V. A. ; Tishchenko, A. V.: Total reflection of light from a corrugated surface of a dielectric waveguide. In: Soviet Journal of Quantum Electronics 15 (1985), Nr. 7, S. 886-887

$\rightarrow$ Zitiert auf den Seiten 1, 3, 4, 5 und 23.

\section{GoOs und HÄNCHEN 1947}

Goos, Fritz ; HÄnchen, Hilda: Ein neuer und fundamentaler Versuch zur Totalreflexion. In: Annalen der Physik 436 (1947), Nr. 7-8, S. 333-346

$\rightarrow$ Zitiert auf Seite 15.

\section{Goos und LINDBERG-HÄNCHEN 1949}

Goos, Fritz ; LindberG-HÄnchen, Hilda: Neumessung des Strahlversetzungseffektes bei Totalreflexion. In: Annalen der Physik 440 (1949), Nr. 3-5,

S. $251-252$

$\rightarrow$ Zitiert auf Seite 15 .

\section{GÖPPERT 1929}

GÖPPERT, Maria: Über die Wahrscheinlichkeit des Zusammenwirkens zweier Lichtquanten in einem Elementarakt. In: Die Naturwissenschaften 17 (1929), Nr. 48, S. 932

$\rightarrow$ Zitiert auf Seite 110

\section{GÖPPERT-MAYER 1931}

GÖPPERT-MAYER, Maria: Über Elementarakte mit zwei Quantensprüngen. In: Annalen der Physik 401 (1931), Nr. 3, S. 273-294

$\rightarrow$ Zitiert auf Seite 110 . 


\section{GRINVALD u. a. 2008}

Grinvald, E. ; Katchalski, T. ; Soria, S. ; Levit, S. ; Friesem, A. A.: Role of photonic bandgaps in polarization-independent grating waveguide structures. In: Journal of the Optical Society of America A 25 (2008), Nr. 6, S. $1435-1443$

$\rightarrow$ Zitiert auf Seite 132

\section{GUENTHER 1982}

Guenther, Karl H.: Columnar and nodular growth of thin films. In: Hunter, William R. (Hrsg.): Proceedings of SPIE (Thin Film Technologies, Arlington, Virginia, USA, 6.-7. Mai 1982). Bd. 346 : Thin Film Technologies and Special Applications. Bellingham (Washington) : SPIE Press, 1982. - ISBN 0-89252381-6, S. 9-18

$\rightarrow$ Zitiert auf Seite 53

\section{HEGER 2005}

HEGER, Petra: Spektrale Eigenschaften nanostrukturierter dielektrischer und metallischer Dünnschichtsysteme. Jena, Universität, PhysikalischAstronomische Fakultät, Diss., 2005

$\rightarrow$ Zitiert auf den Seiten 42 und 53 .

\section{Hessel und Oliner 1965}

Hessel, Alexander ; Oliner, Arthur A.: A new theory of Wood's anomalies on optical gratings. In: Applied Optics 4 (1965), Nr. 10, S. 1275-1297

$\rightarrow$ Zitiert auf den Seiten 4 und 22

\section{HiMMLER 2006}

Himmler, Andreas: Analyse kurzer dielektrischer Wellenleitergitter mit der Methode der finiten Elemente. Paderborn, Universität, Diss., 2006. - ISBN 3-8322-5763-2

$\rightarrow$ Zitiert auf Seite 42 .

HUNSPERGER 2002

Hunsperger, Robert G.: Integrated Optics : Theory and Technology. 5. Aufl. Berlin [u.a.] : SpRINGER-Verlag, 2002 (Advanced Texts in Physics). - ISBN 3-540-43341-4

$\rightarrow$ Zitiert auf den Seiten 17 und 18 . 


\section{Hutley und BiRd 1973}

Hutley, M. C. ; BiRd, V. M.: A Detailed Experimental Study of the Anomalies of a Sinusoidal Diffraction Grating. In: Optica Acta 20 (1973), Nr. 10, S. $771-782$

$\rightarrow$ Zitiert auf Seite 4

\section{IHLEMANN u. a. 2003}

Ihlemann, J. ; Müller, S. ; Puschmann, S. ; Schäfer, D. ; Wei, M. ; Li, J. ; Herman, P. R.: Fabrication of submicron gratings in fused silica by $\mathrm{F}_{2}$-laser ablation. In: Applied Physics A : Materials Science 83 Processing 76 (2003), Nr. 5, S. 751-753

$\rightarrow$ Zitiert auf den Seiten 7 und 48

\section{IMBERT 1972}

IMBERT, Christian: Calculation and experimental proof of the transverse shift induced by total internal reflection of a circularly polarized light beam. In: Physical Review D 5 (1972), Nr. 4, S. 787-796

$\rightarrow$ Zitiert auf Seite 16.

\section{IMBERT und LEVY 1975}

Imbert, Christian ; Levy, Yves: Displacement of a totally reflected light beam: filtering of he polarization states and amplification. In: Nouvelle Revue d'Optique 6 (1975), Nr. 5, S. 285-296

$\rightarrow$ Zitiert auf den Seiten 15 und 16 .

\section{JACKSON 2006}

JACKSOn, John D.: Klassische Elektrodynamik. 4., überarb. Aufl. / Dt. Übers.: Kurt Müller (Übers.) ; Christopher Witte (Bearb.). Berlin [u. a.] : Walter de GRUYTER-Verlag, 2006. - ISBN 3-11-018970-4

$\rightarrow$ Zitiert auf Seite 9

JАCOB u. a. 2000

Jacob, Donald K. ; Dunn, Steven C. ; Moharam, M. G.: Design considerations for narrow-band dielectric resonant grating reflection filters of finite length. In: Journal of Optical Society of America A 17 (2000), Nr. 7, S. 1241-1249

$\rightarrow$ Zitiert auf den Seiten 3, 5, 26, 31 und 36. 


\section{JАCOB u. a. 2001a}

Jacob, Donald K. ; Dunn, Steven C. ; Moharam, M. G.: Interference approach applied to dual-grating dielectric resonant grating reflection filters. In: Optics Letters 26 (2001), Nr. 22, S. 1749-1751

$\rightarrow$ Zitiert auf den Seiten 5, 7] und 32 .

\section{JАCOB u. a. 2001b}

Jacob, Donald K. ; Dunn, Steven C. ; Moharam, M. G.: Normally incident resonant grating reflection filters for efficient narrow-band spectral filtering of finite beams. In: Journal of Optical Society of America A 18 (2001), Nr. 9, S. 2109-2120

$\rightarrow$ Zitiert auf den Seiten 5,33 und 36 .

\section{JIN 2002}

Jin, Jianming: The Finite Element Method in Electromagnetics. 2. Aufl. New York : John WiLEY \& Sons, 2002. - ISBN 0-471-43818-9

$\rightarrow$ Zitiert auf Seite 38

\section{KAPPEL u. a. 2004a}

Kappel, C. ; Selle, A. ; Bader, M. A. ; Marowsky, G.: Ultrashort-pulse behavior in resonant reflection filters. In: Armitage, J. C. (Hrsg.) ; Fafard, S. (Hrsg.) ; Lessard, R. A. (Hrsg.) ; Lampropoulos, G. A. (Hrsg.): Proceedings of SPIE (Photonics North 2004, Ottawa, Ontario, Kanada, 27.-29. September 2004). Bd. 5577 : Optical Components and Devices. Bellingham (Washington) : SPIE Press, Dezember 2004. - ISBN 0-8194-5533-4, S. 716-723

$\rightarrow$ Zitiert auf Seite 97

\section{KAPPEL u. a. 2004b}

Kappel, C. ; Selle, A. ; Bader, M. A. ; Marowsky, G.: Resonant doublegrating waveguide structures as inverted Fabry-Perot interferometers. In: Journal of Optical Society of America B 21 (2004), Nr. 6, S. 1127-1136

$\rightarrow$ Zitiert auf den Seiten 3, 32 und 37. 
KAPPEL u. a. 2004c

Kappel, C. ; Selle, A. ; Fricke-Begemann, T. ; Bader, M. A. ; MarowSKY, G.: Giant enhancement of two-photon fluorescence induced by resonant double grating waveguide structures. In: Applied Physics B : Lasers and Optics 79 (2004), Nr. 5, S. 531-534

$\rightarrow$ Zitiert auf Seite 118

\section{KAPPEL u. a. 2005}

Kappel, C. ; Selle, A. ; Fricke-Begemann, T. ; Bader, M. A. ; MAROWSKY, G.: Double grating waveguide structures : 350-fold enhancement of two-photon fluorescence applying ultrashort pulses. In: MorEno-Bond, M. C. (Hrsg.) ; Orellana, G. (Hrsg.): Sensors and Actuators B : Chemical (Proceedings of the 7th European Conference on Optical Chemical Sensors and Biosensors - EUROPT(R)ODE VII, Madrid, Spanien, 4.-7. April 2004). Bd. 107, Nr. 1. Amsterdam : North-Holland/Elsevier Press, Mai 2005. - ISSN 0925-4005, S. 135-139

$\rightarrow$ Zitiert auf Seite 118

\section{KATCHALSKI u. a. 2005a}

Katchalski, T. ; Levy-Yurista, G. ; Friesem, A. A. ; Martin, G. ; HierLE, R. ; ZYSS, J.: Light modulation with electro-optic polymer-based resonant grating waveguide structures. In: Optics Express 13 (2005), Nr. 12, S. 46454650

$\rightarrow$ Zitiert auf Seite 45 .

\section{KATCHALSKI u. a. 2005b}

Katchalski, T. ; Soria, S. ; Teitelbaum, E. ; Friesem, A. A. ; MarowSKY, G.: Two photon fluorescence sensors based on resonant grating waveguide structures. In: Sensors and Actuators B : Chemical (Proceedings of the 7th European Conference on Optical Chemical Sensors and Biosensors - EUROPT(R)ODE VII, Madrid, Spanien, 4.-7. April 2004) 107 (2005), Nr. 1, S. $121-125$

$\rightarrow$ Zitiert auf den Seiten 113,119 und 148 . 


\section{KATCHALSKI 2002}

KATCHALSKI, Tsvi: Resonant grating waveguide structures : Active and passive polymer based and two-dimensional configurations. Rehovot, Isreal, WEIZmann Institute of Science, Diss., 2002

$\rightarrow$ Zitiert auf Seite 6 .

\section{KOGELNIK 1979}

Kogelnik, Herwig: Theory of Dielectric Waveguides. In: TAMIR, Theodor (Hrsg.): Topics in Applied Optics. Bd. 7 : Integrated Optics. 2., überarb. und erw. Aufl. Berlin, Heidelberg: Springer-Verlag, 1979, Kapitel 2. - ISBN 3-540-09673-6, S. 13-81

$\rightarrow$ Zitiert auf Seite 20 .

\section{KOGELNIK 1990}

Kogelnik, Herwig: Theory of Optical Waveguides. In: TAMIR, Theodor (Hrsg.): Springer Series in Electronics and Photonics. Bd. 26 : Guided-Wave Optoelectronics. 2. Aufl. Berlin, Heidelberg : SpRINGER-Verlag, 1990, Kapitel 2. - ISBN 3-540-52780-X, S. 7-88

$\rightarrow$ Zitiert auf Seite 20 .

\section{KuBIN und FletCher 1982}

Kubin, Robert F. ; Fletcher, Aaron N.: Fluorescence quantum yields of some rhodamine dyes. In: Journal of Luminescence 27 (1982), Nr. 4, S. 455462

$\rightarrow$ Zitiert auf Seite 112

\section{KunZ u. a. 1996}

Kunz, R. E. ; DüBendorfer, J. ; Morf, R. H.: Finite grating depth effects for integrated optical sensors with high sensitivity. In: Biosensors \&6 Bioelectronics 11 (1996), Nr. 6-7, S. 653-667

$\rightarrow$ Zitiert auf den Seiten 6 und 26 .

\section{LACOUR u. a. 2003}

Lacour, D. ; Granet, G. ; Plumey, J. ; Mure-Ravaud, A.: Polarization independence of a one-dimensional grating in conical mounting. In: Journal of Optical Society of America A 20 (2003), Nr. 8, S. 1546-1552

$\rightarrow$ Zitiert auf Seite 132 
LACOUR u. a. 2001

Lacour, D. ; Plumey, J.-P. ; Granet, G. ; Ravaud, A. M.: Resonant waveguide grating: Analysis of polarization independent filtering. In: Optical and Quantum Electronics 33 (2001), Nr. 4-5, S. 451-470

$\rightarrow$ Zitiert auf Seite 132 .

\section{LAKOWICZ 2006}

Lakowicz, Joseph R.: Principles of Fluorescence Spectroscopy. 3. Aufl. Berlin [u. a.] : SPRINGER-Verlag, 2006. - ISBN 0-387-31278-1

$\rightarrow$ Zitiert auf den Seiten 111 und 115.

\section{LAUTERBORN und KURZ 2003}

Lauterborn, Werner ; Kurz, Thomas: Coherent Optics : Fundamentals and Applications. Berlin [u. a.] : SpRINGER-Verlag, 2003. - ISBN 3-540-43933-1

$\rightarrow$ Zitiert auf Seite 66.

\section{LAUTERBORN u. a. 1993}

Lauterborn, Werner ; Kurz, Thomas ; Wiesenfeldt, Martin: Kohärente Optik : Grundlagen für Physiker und Ingenieure. Berlin [u.a.] : SPRINGERVerlag, 1993. - ISBN 3-540-56769-0

$\rightarrow$ Zitiert auf den Seiten 10, 37, 94, 98 und 100 .

\section{LEITEL U. a. 2006}

Leitel, R. ; Heger, P. ; Stenzel, O. ; Kaiser, N.: Theoretical treatment of thermal shifts in resonant grating waveguide structures. In: Journal of Optics A : Pure and Applied Optics 8 (2006), Nr. 3, S. 333-337

$\rightarrow$ Zitiert auf den Seiten 7, 33, 34 und 107.

\section{LEITEL u. a. 2007}

Leitel, R. ; Stenzel, O. ; Kaiser, N. ; Tünnermann, A.: Comparison of thermal shifts in resonant grating waveguide structures and multilayer stacks. In: Proceedings of OSA (Optical Interference Coatings - OIC 200\%, Tucson, Arizona, USA, 3.-8. Juni 2007), OSA Press, 2007. - ISBN 1-55752-841-1. Technical Digest CD-Rom, Art. WD2

$\rightarrow$ Zitiert auf Seite 90 . 


\section{LEVENBERG 1944}

LevenberG, Kenneth: A Method for the Solution of Certain Non-Linear Problems in Least Squares. In: The Quarterly of Applied Mathematics 2 (1944),

Nr. 2, S. $164-168$

$\rightarrow$ Zitiert auf Seite 95 .

\section{LEVY-YURISTA und FRIESEM 2000}

Levy-Yurista, Guy ; Friesem, Asher A.: Very narrow spectral filters with multilayered grating-waveguide structures. In: Applied Physics Letters 77 (2000), Nr. 11, S. 1596-1598

$\rightarrow$ Zitiert auf Seite 6 .

\section{LEVY-YuRista und Friesem 2001}

Levy-Yurista, Guy ; Friesem, Asher A.: Dual spectral filters with multilayered grating waveguide structures. In: Applied Physics B : Lasers and Optics 72 (2001), Nr. 8, S. 921-925

$\rightarrow$ Zitiert auf Seite 6 .

\section{LI u. a. 2002}

Li, J. ; Herman, P. R. ; Wei, X M. ; Chen, K. P. ; Ihlemann, J. ; Marowsky, G. ; Oesterlin, P. ; Burghardt, B.: High-resolution $\mathrm{F}_{2}$-laser machining of micro-optic components. In: SugiokA, K. (Hrsg.) ; Gower, M. C. (Hrsg.) ; Haglund, R. F. (Hrsg.) ; Pique, A. (Hrsg.) ; Traeger, F. (Hrsg.) ; Dubowski, J. J. (Hrsg.) ; Hoving, W. (Hrsg.): Proceedings of SPIE (Photonics West 2002: High-Power Lasers and Applications, San José, Kalifornien, USA, 21. Januar 2002). Bd. 4637 : Photon Processing in Microelectronics and Photonics. Bellingham (Washington) : SPIE Press, Juli 2002.

- ISBN 0-8194-4422-7, S. 228-234

$\rightarrow$ Zitiert auf Seite 49 .

\section{1995}

LI, Lifeng: Analysis of planar waveguide grating couplers with double surface corrugations of identical period. In: Optics Communications 114 (1995), Nr. 5-6, S. 406-412

$\rightarrow$ Zitiert auf Seite 85. 


\section{LIU u. a. 1998}

Liu, Z. S. ; Tibuleac, S. ; Shin, D. ; Young, P. P. ; Magnusson, R.: Highefficiency guided-mode resonance filter. In: Optics Letters 23 (1998), Nr. 19, S. $1556-1558$

$\rightarrow$ Zitiert auf den Seiten 5 und 7

\section{LoEWen und Popov 1997}

Loewen, Erwin G. ; Popov, Evgeny ; Thompson, Brian J. (Hrsg.): Optical Engineering. Bd. 58: Diffraction Gratings and Applications. New York : Marcel Dekker, 1997. - ISBN 0-8247-9923-2

$\rightarrow$ Zitiert auf Seite 20.

\section{Lotsch 1968}

Lotsch, Helmut K. V.: Reflection and Refraction of a Beam of Light at a Plane Interface. In: Journal of Optical Society of America 58 (1968), Nr. 4, S. $551-561$

$\rightarrow$ Zitiert auf Seite 16 .

\section{LotsCH 1970}

Lotsch, Helmut K. V.: Beam displacement at total reflection: The GoosHänchen effect, I / II. In: Optik 32 (1970), Nr. 2 / 3, S. 116-137/ 190-204

$\rightarrow$ Zitiert auf Seite 16 .

\section{LotsCH 1971}

Lotsch, Helmut K. V.: Beam displacement at total reflection: The GoosHänchen effect, III / IV. In: Optik 32 (1971), Nr. 4 / 6, S. 299-319 / 553-569

$\rightarrow$ Zitiert auf Seite 16 .

\section{MAgnusson u. a. 1998}

Magnusson, Robert ; Shin, Dongho ; LiU, Zhongshan: Guided-mode resonance Brewster filter. In: Optics Letters 23 (1998), Nr. 8, S. 612-614

$\rightarrow$ Zitiert auf Seite 5 .

\section{MAgNusson und WANG 1992}

Magnusson, Robert ; WAng, Shu-Shaw: New principle for optical filters. In: Applied Physics Letters 61 (1992), Nr. 9, S. 1022-1024

$\rightarrow$ Zitiert auf den Seiten 3,5 und 33 . 


\section{MAgNuSson und WANG 1993}

Schutzrecht US 5216680 (Juni 1993). Magnusson, Robert ; Wang, Shu-Shaw (Erfinder); Board of Regents, The University of Texas System (Anmelder). Pr.: 07/728 673 1991-07-11 - Optical guided-mode resonance filter

$\rightarrow$ Zitiert auf Seite 5 .

\section{MarCUSE 1991}

Marcuse, Dietrich: The Asymmetric Slab Waveguide. In: LiaO, Paul F. (Hrsg.) ; Kelley, Paul L. (Hrsg.): Theory of Dielectric Optical Waveguides. 2. Aufl. Boston [u. a.] : Academic Press, 1991 (Quantum Electronics — Principles and Applications Series), Kapitel 1. - ISBN 0-12-470951-6, S. 1-59

$\rightarrow$ Zitiert auf den Seiten 18 und 20.

MARowsKy u. a. 2004

Marowsky, G. ; Bader, M. A. ; Selle, A. ; Kappel, C. ; FrickeBegemann, T. ; Meinertz, J. ; Ihlemann, J.: Neue Perspektiven der ZweiPhotonen-Technologie. In: Beckmann, D. (Hrsg.) ; Meister, M. (Hrsg.) ; Institut für Bioprozess- und Analysenmesstechnik e. V. (Veranst.): Technische Systeme für Biotechnologie und Umwelt (Tagungsband des 12. Heiligenstädter Kolloquium, Heiligenstadt, Deutschland, 27.-29. September 2004). Heiligenstadt, 2004. - ISBN 3-00-015042-0, S. 119-124

$\rightarrow$ Zitiert auf Seite 118

\section{MARQUARDT 1963}

Marquardt, Donald W.: An Algorithm for Least-Squares Estimation of Nonlinear Parameters. In: Journal of the Society for Industrial and Applied Mathematics 11 (1963), Nr. 2, S. 431-441

$\rightarrow$ Zitiert auf Seite 95 .

\section{MASHEV und Popov 1984}

Mashev, Lyuben ; Popov, Evgeny: Diffraction efficiency anomalies of multicoated dielectric gratings. In: Optics Communications 51 (1984), Nr. 3, S. $131-136$

$\rightarrow$ Zitiert auf Seite 4 


\section{MASHev und Popov 1985}

Mashev, Lyuben ; Popov, Evgeny: Zero order anomaly of dielectric coated gratings. In: Optics Communications 55 (1985), Nr. 6, S. 377-380

$\rightarrow$ Zitiert auf Seite 5

\section{MAXWELL 1865}

MAXWELL, James C.: A dynamical theory of the electromagnetic field. In: Philosophical Transactions of the Royal Society of London 155 (1865), S. 459512

$\rightarrow$ Zitiert auf Seite 9

\section{MOHARAM u. a. 1995a}

Moharam, M. G. ; Grann, E. B. ; Pommet, D. A. ; Gaylord, T. K.: Formulation for stable and efficient implementation of the rigorous coupledwave analysis of binary gratings. In: Journal of Optical Society of America A 12 (1995), Nr. 5, S. 1068-1076

$\rightarrow$ Zitiert auf Seite 38.

\section{MOHARAM u.a. $1995 \mathrm{~b}$}

Moharam, M. G. ; Pommet, D. A. ; Grann, E. B. ; Gaylord, T. K.: Stable implementation of the rigorous coupled-wave analysis for surface-relief gratings: enhanced transmittance matrix approach. In: Journal of Optical Society of America A 12 (1995), Nr. 5, S. 1077-1086

$\rightarrow$ Zitiert auf Seite 38 .

\section{Moulton 1982}

Moulton, Peter F.: Ti-doped sapphire: tunable solid-state laser. In: Optics News 8 (1982), Nr. 6, S. 9

$\rightarrow$ Zitiert auf Seite 74 .

\section{NEUSCHÄFER u. a. 2003}

Neuschäfer, D. ; Budach, W. ; Wanke, C. ; Chibout, S.: Evanescent resonator chips: a universal platform with superior sensitivity for fluorescencebased microarrays. In: Biosensors \& Bioelectronics 18 (2003), Nr. 4, S. 489497

$\rightarrow$ Zitiert auf den Seiten 7, 8, 110 und 122 . 


\section{NeVIERE u. a. 1977}

Neviere, M. ; Maystre, D. ; Vincent, P.: Determination of the leaky modes of a corrugated waveguide: application to the study of anomalies of dielectric coated gratings. In: Journal of Optics 8 (1977), Nr. 4, S. 231-242

$\rightarrow$ Zitiert auf Seite 23

\section{NeVIÈRE u. a. 1995}

Nevière, M. ; Popov, E. ; Reinisch, R.: Electromagnetic resonances in linear and nonlinear optics: phenomenological study of grating behavior through the poles and zeros of the scattering operator. In: Journal of Optical Society of America A 12 (1995), Nr. 3, S. 513-523

$\rightarrow$ Zitiert auf Seite 6 .

\section{NeVIÈRE u. a. 2000}

Nevière, M. ; Popov, E. ; Reinisch, R. ; Vitrant, G. ; Garito, A. F. (Hrsg.) ; Kajzar, F. (Hrsg.) ; Boardman, A. D. (Hrsg.): Advances in Nonlinear Optics. Bd. 5: Electromagnetic resonances in nonlinear optics. Amsterdam [u. a.] : Gordon \& BreACH Science Publishers, 2000. - ISBN 90-5699$317-8$

$\rightarrow$ Zitiert auf Seite 6 .

\section{NeviĚre und Popov 2003}

Nevière, Michel ; Popov, Evgeny: Grating Couplers and Resonant Excitation of Guided Modes. In: Thompson, Brian J. (Hrsg.): Optical Engineering. Bd. 81 : Light Propagation in Periodic Media : Differential Theory and Design. New York, Basel : Marcel Dekker, 2003, Kapitel 13. - ISBN 0-8247-0893-8, S. 303-329

$\rightarrow$ Zitiert auf Seite 21.

\section{NIEDERER u. a. 2004}

Niederer, G. ; Herzig, H. P. ; Shamir, J. ; Thiele, H. ; Schnieper, M. ; Zschokke, Ch.: Tunable, oblique incidence resonant grating filter for telecommunications. In: Applied Optics 43 (2004), Nr. 8, S. 1683-1694

$\rightarrow$ Zitiert auf den Seiten 98 und 132 . 
NIEDERER u. a. 2005

Niederer, G. ; Nakagawa, W. ; Herzig, H. P. ; Thiele, H.: Design and characterization of a tunable polarization-independent resonant grating filter. In: Optics Express 13 (2005), Nr. 6, S. 2196-2200

$\rightarrow$ Zitiert auf Seite 132

\section{NiEDERER 2004}

NiEDERER, Guido: Resonant grating filters for microsystems. Neuchâtel, Schweiz, University, Diss., 2004

$\rightarrow$ Zitiert auf Seite 132

\section{NORTON u. a. 1997}

Norton, Scott. M. ; Erdogan, Turan ; Morris, Michael G.: Coupled-mode theory of resonant-grating filters. In: Journal of Optical Society of America A 14 (1997), Nr. 3, S. 629-639

$\rightarrow$ Zitiert auf den Seiten 30 und 36 .

\section{Norton u. a. 1998}

Norton, Scott M. ; Morris, G. M. ; Erdogan, Turan: Experimental investigation of resonant-grating filter lineshapes in comparison with theoretical models. In: Journal of Optical Society of America A 15 (1998), Nr. 2, S. 464472

$\rightarrow$ Zitiert auf Seite 32 .

\section{NoRTON 1997}

Norton, Scott M.: Resonant grating structures : Theory, design, and applications. Rochester, New York, USA, University, Diss., 1997

$\rightarrow$ Zitiert auf Seite 30.

\section{Novotny und Hecht 2006}

Novotny, Lukas ; Hecht, Bert: Near-field optical probes. In: Principles of Nano-Optics. Cambridge [u. a.] : Cambridge University Press, 2006, Kapitel 6.

- ISBN 0-521-83224-1, S. 173-224

$\rightarrow$ Zitiert auf den Seiten 15$]$ und 120 . 


\section{PAWLOWSKI u. a. 2000}

Pawlowski, E. ; Friesem, A. A. ; Kuller, L. ; Levy-Yurista, G. ; LudWig, R. ; Weber, H. G.: Femtosecond pulse source with grating-waveguide structure. In: LI, T. (Hrsg.): Proceedings of OSA (Diffractive Optics and MicroOptics - DOMO 2000, Québec, Québec, Kanada, 18.-22. Juni 2000), OSA Press, 2000 (OSA Trends in Optics and Photonic Series 41). - Technical Digest CD-Rom. - ISBN 1-557-52636-2, Art. DThD5

$\rightarrow$ Zitiert auf den Seiten 6 und 107.

\section{Pereira u. a. 2002}

Pereira, S. ; Sipe, J. E. ; Bader, M. A. ; Soria, S. ; Marowsky, G.: Losstolerant, narrow-band reflector in the $\mathrm{UV}$ using a grating-waveguide structure. In: Applied Physics B : Lasers and Optics 75 (2002), Nr. 6-7, S. 635-640

$\rightarrow$ Zitiert auf Seite 105 .

\section{PÖHLMANN u.a. 2004}

Pöhlmann, T. ; Böckmann, R. A. ; Grubmüller, H. ; UchanskaZiegler, B. ; Ziegler, A. ; Alexiev, U.: Differential peptide dynamics is linked to MHCpolymorphism. In: Journal of Biological Chemistry 279 (2004), Nr. 27, S. 28197-28201

$\rightarrow$ Zitiert auf Seite 126 .

\section{Pissadakis u. a. 2004}

Pissadakis, S. ; Zervas, M. N. ; Reekie, L. ; Wilkinson, J. S.: Highreflectivity Bragg gratings fabricated by 248-nm excimer laser holographic ablation in thin $\mathrm{Ta}_{2} \mathrm{O}_{5}$ films overlaid on glass waveguides. In: Applied Physics A : Materials Science 8 Processing 79 (2004), Nr. 4-6, S. 1093-1096

$\rightarrow$ Zitiert auf Seite 48 .

\section{PULKer 2003}

Pulker, Hans K.: Film Deposition Methods. In: Kaiser, Norbert (Hrsg.) ; Pulker, Hans K. (Hrsg.): Springer Series in Optical Sciences. Bd. 88 : Optical Interference Coatings. Berlin, Heidelberg : SPRINGER-Verlag, 2003. - ISBN 3540-00364-9, S. 131-153

$\rightarrow$ Zitiert auf Seite 53 . 


\section{RAMSDEN 1997}

RAMSDEn, Jeremy J.: Optical Biosensors. In: Journal of Molecular Recognition 10 (1997), S. 109-120

$\rightarrow$ Zitiert auf Seite 109

\section{RAYLEIGH 1907}

RAYLEIGH, Lord: Note on the remarkable case of diffraction spectrum described by Prof. Wood. In: Philosophical Magazine 14 (1907), Nr. 79, S. 60-65

$\rightarrow$ Zitiert auf den Seiten 4 und 22

\section{Rosenblatt u.a. 1997}

Rosenblatt, David ; Sharon, Avner ; Friesem, Asher A.: Resonant Grating Waveguide Structures. In: IEEE Journal Of Quantum Electronics 33 (1997), Nr. 11, S. 2038-2059

$\rightarrow$ Zitiert auf den Seiten 2, 6, 25, und 35.

\section{RuMPF und JoHnson 2007}

Rumpf, Raymond C. ; Johnson, Eric G.: Modeling fabrication to accurately place GMR resonances. In: Optics Express 15 (2007), Nr. 6, S. 3452-3464

$\rightarrow$ Zitiert auf den Seiten 42 und 44.

\section{RYTOV 1956}

RYTOV, Sergei M.: Electromagnetic properties of a finely stratified medium. In: Soviet Physics JETP 2 (1956), Nr. 3, S. 466-475

$\rightarrow$ Zitiert auf Seite 26.

\section{SELLE u. a. 2005}

Selle, A. ; Kappel, C. ; Bader, M. A. ; Marowsky, G. ; Winkler, K. ; Alexiev, U.: Picosecond-pulse-induced two-photon fluorescence enhancement in biological material by application of grating waveguide structures. In: Optics Letters 30 (2005), Nr. 13, S. 1683-1685

$\rightarrow$ Zitiert auf Seite 127

\section{SELLE 2003}

Selle, André: Gitter-Wellenleiter-Strukturen in der modernen Optik. Göttingen, Universität, Diplomarbeit, Oktober 2003

$\rightarrow$ Zitiert auf den Seiten 24, 25, 32, 33, 37, 40, und 98 . 


\section{SHARON u. a. 1997a}

Sharon, A. ; Glasberg, S. ; Rosenblatt, D. ; Friesem, A. A.: Metalbased resonant grating-waveguide structures. In: Journal of Optical Society of America A 14 (1997), Nr. 3, S. 588-595

$\rightarrow$ Zitiert auf Seite 5 .

\section{SHARON u. a. 1996a}

Sharon, A. ; Rosenblatt, D. ; Friesem, A. A. ; Weber, H. G. ; Engel, H. ; Steingrueber, R.: Light modulation with resonant grating-waveguide structures. In: Optics Letters 21 (1996), Nr. 19, S. 1564-1566

$\rightarrow$ Zitiert auf den Seiten 5 und 45

\section{SHARON u. a. 1995}

Sharon, A. ; Rosenblatt, D. J. ; Friesem, A. A. ; Weber, H.-G. ; Engel, H. ; Steingrueber, R.: Resonance phenomena in grating/waveguide structures. In: Shladov, I. (Hrsg.) ; Wiessman, Y. (Hrsg.) ; Kopeika, N. (Hrsg.): Proceedings of SPIE (9th Meeting on Optical Engineering in Israel, Tel-Aviv, Israel, 24.-26. Oktober 1994). Bd. 2426 : Optical Components and Techniques. Bellingham (Washington) : SPIE Press, 1995. - ISBN 0-8194-1774-2, S. 416422

$\rightarrow$ Zitiert auf den Seiten 2, 3, 5 und 36.

\section{SHARON u. a. $1996 b$}

Sharon, Avner ; Rosenblatt, David ; Friesem, Asher A.: Narrow spectral bandwidths with grating waveguide structures. In: Applied Physics Letters 69 (1996), Nr. 27, S. 4154-4156

$\rightarrow$ Zitiert auf Seite 6 .

\section{SHARON u. a. 1997b}

Sharon, Avner ; Rosenblatt, David ; Friesem, Asher A.: Resonant grating-waveguide structures for visible and near-infrared radiation. In: Journal of Optical Society of America A 14 (1997), Nr. 11, S. 2985-2993

$\rightarrow$ Zitiert auf den Seiten 32 und 35 . 


\section{SÁNCHEZ u.a. 1997}

SÁnchez, E. J. ; Novotny, L. ; Holtom, G. R. ; Xie, X. S.: RoomTemperature Fluorescence Imaging and Spectroscopy of Single Molecules by Two-Photon Excitation. In: Journal of Physical Chemistry A : Molecules, Spectroscopy, Kinetics, Environment \&6 General Theory 101 (1997), Nr. 38, S. 7019-7023

$\rightarrow$ Zitiert auf Seite 125

\section{SOMMERFELD 1964}

Sommerfeld, Arnold: Vorlesungen über theoretische Physik. Bd. 4: Optik. 3., durchges. Aufl. / rev. von Fritz Bopp (Rev.) ; Josef Meixner (Rev.). Leipzig : Akademische Verlagsgesellschaft GeEst \& PorTig, 1964

$\rightarrow$ Zitiert auf Seite 22.

\section{SORIA u. a. 2004a}

Soria, S. ; Katchalski, T. ; Teitelbaum, E. ; Friesem, A. A. ; MarowSKY, G.: Enhanced two-photon fluorescence excitation by resonant grating waveguide structures. In: Optics Letters 29 (2004), Nr. 17, S. 1989-1991

$\rightarrow$ Zitiert auf Seite 119

\section{SORIA u. a. $2004 b$}

Soria, S. ; Katchalski, T. ; Teitelbaum, K. ; Friesem, A. A.: Grating Waveguide Structures for Nonlinear Optics and Biotechnology / LaserLaboratorium Göttingen e. V., Göttingen, Deutschland ; Weizmann Institute of Science, Rehovot, Israel. 2004 (Förderkennzeichen 13 N 7963). - Forschungsbericht. - Vorhaben im BMBF VDI-Forscherverbund: German Israeli Cooperation in Ultrafast Laser Technologies - GILCULT, Abschlussbericht

$\rightarrow$ Zitiert auf Seite 131.

\section{SORIA u. a. 2005}

Soria, S. ; Thayil, A. ; Badenes, G. ; Bader, M. A. ; Selle, A. ; MAROWsky, G.: Resonant double grating waveguide structures as enhancement platforms for two-photon fluorescence excitation. In: Applied Physics Letters 87 (2005), Nr. 8. - Art. 081109

$\rightarrow$ Zitiert auf Seite 119 . 


\section{STENZEL 1996}

Stenzel, Olaf: Das Dünnschichtspektrum : Ein Zugang von den Grundlagen zur Spezialliteratur. Berlin : Akademie Verlag, 1996. - ISBN 3-05-501728-5

$\rightarrow$ Zitiert auf den Seiten 83 und 102

\section{STENZEL 2003}

Stenzel, Olaf: New Challenges in Optical Coating Design. In: Kramer, Bernhard (Hrsg.): Advances in Solid State Physics. Bd. 43. Berlin : SpringerVerlag, 2003. - ISBN 3-540-40150-4, S. 875-887

$\rightarrow$ Zitiert auf Seite 24

\section{STENZEL 2004}

STENZEL, Olaf: Resonant reflection and absorption in grating waveguide structures. In: Sidorin, Yakov (Hrsg.) ; Tervonen, Ari (Hrsg.): Proceedings of SPIE (Photonics West 2004 : Integrated Optoelectronic Devices, San José, Kalifornien, USA, 24.-29. Januar 2004). Bd. 5355 : Integrated Optics : Devices, Materials, and Technologies VIII. Bellingham (Washington) : SPIE Press, 2004. - ISBN 0-819-45276-9, S. 1-13

$\rightarrow$ Zitiert auf Seite 34

\section{STENZEL 2005}

Stenzel, Olaf: Extended Detail: Remarks on Resonant Grating Waveguide Structures. In: ERTL, G. (Hrsg.) ; LÜth, H. (Hrsg.) ; Mills, D. L. (Hrsg.): Springer Series in Surface Sciences. Bd. 44 : The Physics of Thin Film Optical Spectra : An Introduction. Berlin : SPRINGER-Verlag, 2005, Kapitel 9. - ISBN 3-540-23147-1, S. 145-150

$\rightarrow$ Zitiert auf den Seiten 24 und 25 .

\section{STEWART 1981}

STEWART, Walter W.: Lucifer dyes - highly fluorescent dyes for biological tracing. In: Nature 292 (1981), Nr. 5818, S. 17-21

$\rightarrow$ Zitiert auf Seite 113 


\section{STOCKERMANS 1998}

Stockermans, Raymond J.: Resonant Grating Waveguide Structures and their application as high quality narrowband filters. Kingston, Ontario, Canada, Royal Military College of Canada, Diplomarbeit, Mai 1998. - Degree of Master of Engineering

$\rightarrow$ Zitiert auf Seite 6 .

\section{SUZUKI 2007}

Suzuki, Kazuaki: Electron beam lithography systems. In: Suzuki, Kazuaki (Hrsg.) ; Smith, Bruce W. (Hrsg.): Optical Science and Engineering Series. Bd. 126 : Microlithography : Science and Technology. 2. Aufl. CRC Press, 2007, Kapitel 6. - ISBN 0-8247-9024-3, S. 329-360

$\rightarrow$ Zitiert auf Seite 48 .

\section{THAYIL u. a. 2005}

Thayil, A. ; Soria, S. ; Badenes, G. ; Katchalski, T. ; Friesem, A. A. ; Selle, A. ; Bader, M. A. ; Marowsky, G.: Two-photon fluorescence spectroscopy by resonant single and double grating waveguide structures. In: Proceedings of OSA (Conference on Lasers and Electro-Optics - CLEO 2005, Baltimore, Maryland, USA, 22.-27. Mai), OSA Press, 2005. - ISBN 1-55752770-9. - Technical Digest CD-Rom. - ISBN 1-55752-770-9, Art. CTuH2

$\rightarrow$ Zitiert auf Seite 119 .

\section{THIELSCH 2003}

ThIELSCH, Roland: Optical Coatings for the DUV/VUV. In: KAISER, Norbert (Hrsg.) ; Pulker, Hans K. (Hrsg.): Springer Series in Optical Sciences. Bd. 88 : Optical Interference Coatings. Berlin, Heidelberg : Springer-Verlag, 2003. - ISBN 3-540-00364-9, S. 257-279

$\rightarrow$ Zitiert auf Seite 105 .

\section{ThURMAN und MorRIS 2003}

Thurman, Samuel T. ; Morris, G. M.: Controlling the spectral response in guided-mode resonance filter design. In: Applied Optics 42 (2003), Nr. 16, S. $3225-3233$

$\rightarrow$ Zitiert auf den Seiten 33 und 106. 


\section{TiefenthaleR und LuKosz 1989}

Tiefenthaler, Kurt ; Lukosz, Walter: Sensitivity of grating couplers as integrated-optical chemical sensors. In: Journal of Optical Society of America $B 6$ (1989), Nr. 2, S. 209-220

$\rightarrow$ Zitiert auf den Seiten 6 und 109 .

\section{TISHCHENKO u. a. 2003}

Tishchenko, Alexandre V. ; Hamdoun, M. ; Parriaux, Olivier: Twodimensional coupled mode equation for grating waveguide excitation by a focused beam. In: Optical and Quantum Electronics 35 (2003), Nr. 4-5, S. $475-491$

$\rightarrow$ Zitiert auf Seite 98

\section{VoIGT 1911}

VoIGT, Woldemar: Beiträge zu Lord Rayleigh's Theorie der Gitterbeugung. In: Nachrichten von der Gesellschaft der Wissenschaften zu Göttingen 1911 (1911), Nr. 1, S. 41-57

$\rightarrow$ Zitiert auf Seite 4

\section{WANG u. a. 1990}

Wang, S. S. ; Magnusson, R. ; Bagby, J. S. ; Moharam, M. G.: Guidedmode resonances in planar dielectric-layer diffraction gratings. In: Journal of Optical Society of America A 7 (1990), Nr. 8, S. 1470-1474

$\rightarrow$ Zitiert auf den Seiten 3 und 5

\section{WANG und MAGNUSSON 1993}

WAng, Shu-Shaw ; Magnusson, Robert: Theory and applications of guidedmode resonance filters. In: Applied Optics 32 (1993), Nr. 14, S. 2606-2613

$\rightarrow$ Zitiert auf den Seiten 5,33 und 38 .

\section{WAWRO u.a. 2007}

Schutzrecht US 7167615 (Januar 2007). Wawro, Debra D. ; TiBuleAC, Sorin ; Magnusson, Robert (Erfinder); Board of Regents, The UniversiTy OF TeXas System (Anmelder). Pr.: 09/707 435 2000-06-11 - Resonant waveguide-grating filters and sensors and methods for making and using same $\rightarrow$ Zitiert auf Seite 131 


\section{WAWRO u. a. 2008}

Schutzrecht US 7400399 (Juli 2008). Wawro, Debra D. ; TibuleaC, Sorin ; Magnusson, Robert (Erfinder); Board of Regents, The University of Texas System (Anmelder). Pr.: 11/305065 2005-12-16 - Methods for using resonant waveguide-grating filters and sensors

$\rightarrow$ Zitiert auf Seite 131

\section{WEBER u. a. 2004}

Weber, H. G. ; Pawlowski, E. ; Schlüter, S. ; Küller, L. ; Katchalski, T. ; Levy-Yurista, G. ; Friesem, A. A. ; Fraunhofer-Gesellschaft Zur Förderung Der Angewandten Forschung (Hrsg.): Bauelemente für die optische Nachrichtentechnik auf der Basis von Gitter-WellenleiterStrukturen - Devices for Advanced Optical Networks / Fraunhofer-Institut für Nachrichtentechnik, Heinrich-Hertz-Institut, Berlin, Deutschland ; Weizmann Institute of Science, Rehovot, Israel. Berlin, Januar 2004 (Förderkennzeichen 01 BP 703 A). - Forschungsbericht. - Teilvorhaben im BMBF Förderprogramm: Innovative Kommunikationsnetze - KomNet, Abschlussbericht

$\rightarrow$ Zitiert auf den Seiten 6 und 107 .

\section{Weber und PAWLOWSKi 2000}

WeBer, Hans-Georg ; PAWLOWsKi, Edgar: Gitter-Wellenleiter-Struktur für den Einsatz in einem moden-gekoppelten Halbleiterlaser mit Wellenlängendurchstimmbarkeit ohne mechanisch bewegliche Elemente / Heinrich-HertzInstitut für Nachrichtentechnik Berlin GmbH. Berlin, September 2000 (Förderkennzeichen 01 BP 703/6). - Forschungsbericht. - Teilvorhaben im BMBF. Förderprogramm mit Förderschwerpunkt Photonik, Abschlussbericht

$\rightarrow$ Zitiert auf den Seiten 3, 6 und 107.

\section{WEHNER und IHLEMANN 2005}

Wehner, Martin ; Ihlemann, Jürgen: Micro-Machining. In: Basting, Dirk (Hrsg.) ; Marowsky, Gerd (Hrsg.): Excimer Laser Technology. Berlin [u. a.] : Springer-Verlag, 2005, Kapitel 11.2. - ISBN 3-540-20056-8, S. 155-186

$\rightarrow$ Zitiert auf Seite 48 . 


\section{WERNECKE 2003}

WERNECKE, Roland: Industrielle Feuchtemessung : Grundlagen, Messmethoden, technische Anwendungen. Weinheim : WiLEY-VCH Verlag, 2003. - ISBN 3-527-30285-9

$\rightarrow$ Zitiert auf Seite 90

\section{WIKI und KUNZ 2000}

Wiki, Max ; Kunz, Rino E.: Wavelength-interrogated optical sensor for biochemical applications. In: Optics Letters 25 (2000), Nr. 7, S. 463-465

$\rightarrow$ Zitiert auf den Seiten 47 und 109 .

\section{WIKI und MAISENHÖLDER 2004}

Schutzrecht EP 1435009-B1 (Juli 2004). WiKI, Max ; MAISEnHÖLDER, Bernd (Erfinder); OC Oerlikon Balzers AG (Anmelder). Pr.: CH 188701 200110-12. - Lichtkoppelelement

$\rightarrow$ Zitiert auf Seite 47

\section{WoOD 1902}

WooD, Robert W.: On a remarkable case of uneven distribution of light in a diffraction grating spectrum. In: Philosophical Magazine 4 (1902), S. 396-402

$\rightarrow$ Zitiert auf den Seiten 4 und 22

\section{XU und WeBB 1996}

Xu, Chris ; WeBB, Watt W.: Measurement of two-photon excitation cross sections of molecular fluorophores with data from 690 to $1050 \mathrm{~nm}$. In: Journal of Optical Society of America B 13 (1996), Nr. 3, S. 481-491

$\rightarrow$ Zitiert auf den Seiten 112, 113, 121 und 126.

\section{XU und WeBB 1997}

Xu, Chris ; WeBB, Watt W.: Multiphoton Excitation of Molecular Fluorophores and Nonlinear Laser Microscopy. In: LAKOWICZ, Joseph R. (Hrsg.): Topics in Fluorescence Spectroscopy. Bd. 5 : Nonlinear and Two-Photon Induced Fluorescence. New York : Plenum Press, 1997, Kapitel 11. - ISBN 0-306-45553-6, S. $471-540$

$\rightarrow$ Zitiert auf Seite 111 


\section{YARIV und YEH 2003}

YARIV, Amnon ; YeH, Pochi: Nonlinear Optics. In: Optical Waves in Crystals

: Propagation and Control of Laser Radiation. Hoboken (New Jersey) : John Wiley \& Sons, 2003 (Wiley Series in Pure and Applied Optics), Kapitel 12.

- ISBN 0-471-43081-1, S. 504-548

$\rightarrow$ Zitiert auf Seite 67.

\section{Zhu und Cangellaris 2006}

Zhu, Yu ; Cangellaris, Andreas C.: Finite Element Analysis of Periodic Structures. In: Dudley, Donald G. (Hrsg.): Multigrid Finite Element Methods for Electromagnetic Field Modeling. Hoboken (New Jersey) : John WiLEY \& Sons, 2006 (IEEE Press Series on Electromagnetic Wave Theory), Kapitel 12. - ISBN 0-471-74110-8, S. 365-400

$\rightarrow$ Zitiert auf Seite 42 . 


\section{Danksagung}

Bei der Entstehung meiner Doktorarbeit haben mich viele Menschen auf unterschiedliche Art und Weise unterstützt und begleitet. Bei diesen Menschen möchte ich mich ganz herzlich bedanken! Da einzelne Personen einen überaus wichtigen Beitrag für diese Arbeit geleistet haben, möchte ich diese Personen hervorheben und namentlich nennen:

An erster Stelle möchte ich Prof. Dr. Gerd MAROWSKY meinen Dank aussprechen, der mir die Möglichkeit gegeben hat, meine Doktorarbeit am Laser-Laboratorium Göttingen e. V. (LLG) selbständig anzufertigen. Darüber hinaus hat er als Hauptreferent maßgeblich den Grundriss dieser Arbeit mitbestimmt und war dabei stets offen für neue Ideen.

Des Weiteren danke ich Prof. Dr. WERnER LAUTERBORN vom III. Physikalischen Institut für die Übernahme des Zweitgutachtens.

Besonders bedanken möchte ich mich bei DR. MARK ANDREAS BADER für die überaus kompetente Betreuung während dieser Zeit. Dank seiner Anregungen und seiner permanenten Bereitschaft, neue Ideen zu entwickeln, hat er maßgeblich zum Gelingen dieser Arbeit beigetragen.

Ich bedanke mich bei meinem ehemaligen Kollegen DR. CHRISTOPH KAPPEL sowie bei DR. UWE LEINHOS, die stets bemüht waren, neue Impulse und Lösungsvorschläge zu geben. Zudem wurden unter ihrer Anleitung viele Experimente im Labor überhaupt erst möglich.

Allen Mitarbeitern der Abteilung Nanostrukturen unter der Leitung von DR. JÜRGEN IHLEMANN möchte ich an dieser Stelle für ihre stete Hilfsbereitschaft sowie die gute Zusammenarbeit danken.

Besonderer Dank gebührt an dieser Stelle Dr. Thomas FriCKe-BEgemann, der mir stets mit wertvollen Hinweisen und fachlichen Ratschlägen zur Seite stand und durch dessen konstruktive Kritik die Qualität dieser Arbeit gesteigert werden konnte.

Ein Dankeschön geht an DR. JöRG MEINERTZ für die Unterstützung bei der Gliederung des Referats. 
Prof. Dr. UlRike Alexiev und DR. KATHRin WinkLer von der Freien Universität Berlin danke ich für ihre engagierte Zusammenarbeit und den geleisteten Wissenstransfer auf dem Gebiet der Biophysik sowie ihre Unterstützung bei den Experimenten mit biologischen Proben.

Ein Dankeschön geht an die ehemalige LLG-Mitarbeiterin DR. SILVIA SORIA sowie an ihre Kollegin ANISHA THAYIL vom spanischen Instituto de Ciencias Fotónicas (ICFO), deren Kooperationsbereitschaft viele gemeinsame Experimente zur Zwei-Photonen-Fluoreszenz ermöglichte.

Außerdem möchte ich mich bei Dr. Olaf Stenzel und Dr. Petra Heger vom FRAUNHOFER-Institut für Angewandte Optik und Feinmechanik (IOF) für die Herstellung der wellenleitenden Schichten bedanken.

Bedanken möchte ich mich auch bei OlaF MÄDIGER, THOMAS EgGERS und MAIK LÜBBECKE für die umfassende technische Unterstützung.

Für die aufmunternden Worte während der gemeinsamen Doktorandenzeit danke ich meinem Kollegen und Freund RALF MÜLLER sowie meiner Kollegin RONJA BÄUMNER recht herzlich.

Für das Korrekturlesen der Doktorarbeit möchte ich mich bei JANA ZOUFAHL und THOMAS LAMBRECHT bedanken.

Für ihre Geduld und Unterstützung während der gesamten Promotionszeit geht ein ganz Vliches Dankeschön an meine Frau Manuela.

Bedanken möchte ich mich auch bei meinen Kindern LENA und THEO, die mich auf ihre eigene Weise unterstützt haben.

Göttingen, 5. Juni 2009

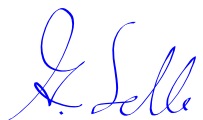




\section{Lebenslauf}

\section{Persönliche Daten}

Name

Geburtsdatum/-ort

Staatsangehörigkeit

Familienstand

\section{Ausbildung}

$07 / 1996$

10/1997 - 09/1998

$10 / 1998-10 / 2003$

$11 / 2003$

$04 / 2004-10 / 2008$

$11 / 2008$

\section{Beruflicher Werdegang}

$01 / 2004-12 / 2005$

$01 / 2006-06 / 2009$
André Selle

8. November 1977, Dessau-Roßlau

deutsch

verheiratet, 2 Kinder

Allgemeine Hochschulreife, Dessau-Roßlau

Studium der Physik, Technische Universität Braunschweig Studium der Physik, Georg-August-Universität Göttingen Abschluss als Diplom-Physiker, Note: ,sehr gut" Promotionsstudiengang Physik, Georg-August-Universität Göttingen

Abschluss der Promotion, Note: „magna cum laude“

Wissenschaftlicher Mitarbeiter im Projekt:

„Entwicklung neuartiger, höchstreflektierender Spiegel für den kurzwelligen Spektralbereich: Superspiegel für die moderne Optik (SPOT)“, Laser-Laboratorium Göttingen e. V.

Wissenschaftlicher Mitarbeiter im Projekt:

„Parallelisierte Fluoreszenz-Korrelations-Spektroskopie mit Einzelmolekül-Analyse für pharmazeutische ScreeningVerfahren und medizinische Diagnostik (FCS-Sensor)“", Laser-Laboratorium Göttingen e. V. 